Louisiana State University

LSU Digital Commons

7-4-2014

\title{
Chemicals Facilitating Reprogramming: Targeting the SAM Binding Site to Identify Novel Methyltransferase Inhibitors
}

\author{
Jeong Do Kim \\ NuPotential, Inc. \\ Jong S. Rim \\ NuPotential, Inc. \\ Robert B. Crochet \\ Louisiana State University \\ Yong Hwan Lee \\ Louisiana State University \\ Jaroslaw Staszkiewicz \\ NuPotential, Inc.
}

See next page for additional authors

Follow this and additional works at: https://digitalcommons.Isu.edu/biosci_pubs

\section{Recommended Citation}

Kim, J., Rim, J., Crochet, R., Lee, Y., Staszkiewicz, J., Gao, R., \& Eilertsen, K. (2014). Chemicals Facilitating Reprogramming: Targeting the SAM Binding Site to Identify Novel Methyltransferase Inhibitors. Chemical Biology in Regenerative Medicine: Bridging Stem Cells and Future Therapies, 163-179. https://doi.org/ 10.1002/9781118695746.ch10

This Article is brought to you for free and open access by the Department of Biological Sciences at LSU Digital Commons. It has been accepted for inclusion in Faculty Publications by an authorized administrator of LSU Digital Commons. For more information, please contact ir@lsu.edu. 


\section{Authors}

Jeong Do Kim, Jong S. Rim, Robert B. Crochet, Yong Hwan Lee, Jaroslaw Staszkiewicz, Ru Gao, and Kenneth J. Eilertsen 


\section{Chemical Biology in Regenerative Medicine}




\section{Chemical Biology in \\ Regenerative Medicine}

Bridging Stem Cells and Future Therapies

Edited by

CHARLES C. HONG

Department of Medicine, Vanderbilt University, USA

ADA S. AO

Department of Medicine, Vanderbilt University, USA

and

JIJUN HAO

College of Veterinary Medicine, Western University of Health Sciences, USA 
This edition first published 2014

(C) 2014 John Wiley \& Sons, Ltd

Registered office

John Wiley \& Sons Ltd, The Atrium, Southern Gate, Chichester, West Sussex, PO19 8SQ, United Kingdom

For details of our global editorial offices, for customer services and for information about how to apply for permission to reuse the copyright material in this book please see our website at www.wiley.com.

The right of the author to be identified as the author of this work has been asserted in accordance with the Copyright, Designs and Patents Act 1988.

All rights reserved. No part of this publication may be reproduced, stored in a retrieval system, or transmitted, in any form or by any means, electronic, mechanical, photocopying, recording or otherwise, except as permitted by the UK Copyright, Designs and Patents Act 1988, without the prior permission of the publisher.

Wiley also publishes its books in a variety of electronic formats. Some content that appears in print may not be available in electronic books.

Designations used by companies to distinguish their products are often claimed as trademarks. All brand names and product names used in this book are trade names, service marks, trademarks or registered trademarks of their respective owners. The publisher is not associated with any product or vendor mentioned in this book.

Limit of Liability/Disclaimer of Warranty: While the publisher and author have used their best efforts in preparing this book, they make no representations or warranties with respect to the accuracy or completeness of the contents of this book and specifically disclaim any implied warranties of merchantability or fitness for a particular purpose. It is sold on the understanding that the publisher is not engaged in rendering professional services and neither the publisher nor the author shall be liable for damages arising herefrom. If professional advice or other expert assistance is required, the services of a competent professional should be sought.

The advice and strategies contained herein may not be suitable for every situation. In view of ongoing research, equipment modifications, changes in governmental regulations, and the constant flow of information relating to the use of experimental reagents, equipment, and devices, the reader is urged to review and evaluate the information provided in the package insert or instructions for each chemical, piece of equipment, reagent, or device for, among other things, any changes in the instructions or indication of usage and for added warnings and precautions. The fact that an organization or Website is referred to in this work as a citation and/or a potential source of further information does not mean that the author or the publisher endorses the information the organization or Website may provide or recommendations it may make. Further, readers should be aware that Internet Websites listed in this work may have changed or disappeared between when this work was written and when it is read. No warranty may be created or extended by any promotional statements for this work. Neither the publisher nor the author shall be liable for any damages arising herefrom.

\section{Library of Congress Cataloging-in-Publication Data}

Chemical biology in regenerative medicine : bridging stem cells and future therapies / edited by

Charles C. Hong, Ada S. Ao, and Jijun Hao.

p. ; cm.

Includes bibliographical references and index.

ISBN 978-1-118-34959-5 (cloth : alk. paper)

I. Hong, Charles C., 1967- editor of compilation. II. Hao, Jijun, editor of compilation.

III. Ao, Ada, editor of compilation.

[DNLM: 1. Stem Cells-chemistry. 2. Myocytes, Cardiac-chemistry. 3. Regenerative Medicine. QU 325] QH588.S83

$616.02^{\prime} 774-\mathrm{dc} 23$

A catalogue record for this book is available from the British Library.

ISBN: 9781118349595

Cover Image: () iStockphoto/faslooff, Sergey Nivens, luismmolina

Set in 10/12pt Times by Aptara Inc., New Delhi, India.

12014 


\section{Contents}

List of Contributors $\quad$ xi

Preface xiii

1 Wnt Signaling in Regulation of Stem Cells 1

David T. Paik and Antonis K. Hatzopoulos

1.1 Overview of Wnt Signaling 1

1.2 Wnt Signaling in Embryonic Stem Cells 2

1.3 Wnt Signaling in Cardiovascular Progenitor Cells and

1.4 Wnt Signaling in Mesenchymal Stem Cells 5

1.5 Wnt Signaling in Hematopoiesis and Hematopoietic Stem Cells 7

$\begin{array}{lll}1.6 & \text { Wnt Signaling in Neural Stem Cells } & 8\end{array}$

1.7 Wnt Signaling in Endothelial-Mesenchymal Transition 8

1.8 Conclusion 9

$\begin{array}{ll}\text { References } & 10\end{array}$

2 Directed Cardiomyogenesis of Pluripotent Stem Cells 15

Jeffery B. Bylund and Antonis K. Hatzopoulos

$\begin{array}{lll}2.1 & \text { Introduction } & 15\end{array}$

2.2 A Brief Review of Heart Development 16

$\begin{array}{ll}\text { 2.2.1 Cellular and Morphological Movements } & 16\end{array}$

2.2.2 Molecular Events in Heart Development 19

2.2.2.1 Molecular Events of Mesoderm Derivation 19

2.2.2.2 Transcription Factors in Cardiac Development 20

$\begin{array}{ll}\text { 2.2.2.3 Major Developmental Signaling Pathways in Cardiac } & \\ \text { Development } & 22\end{array}$

2.3 Introduction to Pluripotent Stem Cells 23

$\begin{array}{ll}\text { 2.3.1 Unique Features of Pluripotent Stem Cells } & 23\end{array}$

$\begin{array}{ll}\text { 2.3.2 } & \text { Pluripotent Stem Cell Sources } \\ 2.3 .3 & 24\end{array}$

$\begin{array}{ll}\text { 2.3.3 Maintaining Pluripotency } & 24\end{array}$

2.4 Cardiomyocyte Differentiation 25

$\begin{array}{ll}2.4 .1 & \text { Inducing Differentiation } \\ 2.4 .2 & 25\end{array}$

$\begin{array}{ll}\text { 2.4.2 Directed Cardiomyogenesis } & 26\end{array}$

2.5 Conclusion 28

References 
3 Chemical Genetics in Cardiomyocyte Generation

Daqing Jin, Qiao Li, and Tao P. Zhong

3.1 Introduction

3.2 iPSC Generation

3.3 The Chemical Genetics Approach in iPSC Generation 37

3.4 Heart Regeneration $\quad 40$

3.5 The Chemical Genetics Approach in Heart Regeneration 42

3.6 Cardiac Cell Transdifferentiation 43

3.7 Conclusion 44

Acknowledgements $\quad 44$

$\begin{array}{ll}\text { References } & 44\end{array}$

4 Challenges and New Directions for Cardiac Reprogramming 49 Young-Jae Nam and Nikhil Munshi

4.1 Introduction 49

4.2 Strategies for Heart Repair $\quad 49$

4.3 Direct Reprogramming Approaches $\quad 50$

4.4 Current Challenges $\quad 53$

4.5 Conclusion $\quad 56$

Acknowledgements $\quad 56$

$\begin{array}{ll}\text { References } & 56\end{array}$

5 Comparative Analysis of Adult Stem Cell Niches 59

Bryan A. Fioret and Antonis K. Hatzopoulos

5.1 Adult Stem Cells 59

$\begin{array}{lll}5.2 & \text { Adult Stem Cell Niches } & 60\end{array}$

5.3 The Hair Follicle Stem Cell (HFSC) Niche 61

$\begin{array}{lll}5.4 & \text { The Intestinal Stem Cell (ISC) Niche } & 63\end{array}$

5.5 The Hematopoietic Stem Cell (HSC) Niche 66

$\begin{array}{ll}\text { 5.5.1 Endosteal Niche } & 66\end{array}$

$\begin{array}{ll}\text { 5.5.2 Vascular Niche } & 67\end{array}$

$\begin{array}{ll}\text { 5.5.3 Progeny "Niche" } & 68\end{array}$

$\begin{array}{lll}5.6 & \text { The Neural Stem Cell (NSC) Niche } & 68\end{array}$

$\begin{array}{lll}\text { 5.6.1 V-SVZ Niche } & 69\end{array}$

$\begin{array}{ll}\text { 5.6.2 SGZ Niche } & 70\end{array}$

5.7 A Comparison between Tissue-Specific Adult Stem Cell Niches 71

$\begin{array}{lll}5.8 & \text { Future Challenges } & 73\end{array}$

$\begin{array}{ll}\text { Acknowledgements } & 73\end{array}$

$\begin{array}{ll}\text { References } & 73\end{array}$

6 Chemicals and Stem Cells in the Promotion of Regeneration 77

Dikshya Bastakoty, Sarika Saraswati, and Pampee P. Young

6.1 Introduction $\quad 77$

6.2 Biologics in Regenerative Medicine $\quad 78$

6.2.1 Growth Factors and Pro-Angiogenic Agents 78 
6.2.2 Immune-Modulatory Therapies $\quad 79$

$\begin{array}{ll}\text { 6.2.3 Extracellular Matrix-Based Approaches } & 79\end{array}$

$\begin{array}{lll}6.3 & \text { Chemicals and Biomaterials for Healing } & 79\end{array}$

$\begin{array}{lll}6.3 .1 & \text { Small Molecules } & 80\end{array}$

6.3.2 Biomaterial Scaffold and Sustained Delivery $\quad 81$

$\begin{array}{lll}6.4 & \text { Stem-Cell Therapy } & 81\end{array}$

6.4.1 Chemical Manipulation of Stem Cells in Regeneration 82

6.4.2 Embryonic Stem Cells (ESCs) 82

6.4.2.1 Small Molecules for the Culture and Maintenance

6.4.2.2 Small Molecules for ESC Differentiation 83

6.4.3 Induced Pluripotent Stem Cells (iPSCs) 84

$\begin{array}{lll}\text { 6.4.3.1 Generation of iPSCs } & 84\end{array}$

6.4.3.2 Small Molecules that Affect iPSC Epigenomes 84

6.4.3.3 Small Molecules that Affect iPSC Signaling

6.4.4 Mesenchymal Stem Cells (MSCs) $\quad 85$

$\begin{array}{lll}\text { 6.4.4.1 Properties of MSCs } & 85\end{array}$

6.4.4.2 Small Molecules that affect MSC Differentiation $\quad 85$

6.4.4.3 Biopolymers that affect MSC Biology 86

6.4.5 Hematopoietic Stem Cells (HSCs) 86

$\begin{array}{lll}6.5 & \text { Conclusion } & 87\end{array}$

References $\quad 88$

7 Chemically Induced Pluripotent Stem Cells (CiPSCs): A Potential Chemical Biological Breakthrough in Reprogramming?

95

Calvin C. Sheng, Jijun Hao, and Charles C. Hong

7.1 Searching for the "Perfect" Platform 95

7.2 Defining the Advantages of Small Molecules
in Reprogramming

7.3 Understanding the Disadvantages of Using Small Molecules 97

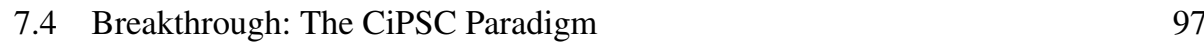

$\begin{array}{lll}7.5 & \text { Conclusion } & 101\end{array}$

$\begin{array}{ll}\text { References } & 101\end{array}$

8 An Introduction to Cellular Reprogramming: The Plasticity of Cell Fates and Identities

Kelly P. Smith, Maria Borowski, and Joseph C. Laning

8.1 Defining Cell Potency

$\begin{array}{ll}8.2 \text { Types of Pluripotent Cell } & 105\end{array}$

$\begin{array}{lll}\text { 8.2.1 Isolated Cell Types } & 105\end{array}$

8.2.1.1 Embryonal Carcinoma Cells 105

8.2.1.2 Embryonic Stem Cells 105

8.2.1.3 Embryonic Germ Cells 105 
8.2.2 Reprogrammed Cell Types 106

8.2.2.1 Cell-Fusion Hybrids 106

8.2.2.2 Somatic Cell Nuclear Transfer Cells 106

8.2.2.3 Induced Pluripotent Stem Cells 106

$\begin{array}{lll}8.3 & \text { Defining Pluripotency } & 107\end{array}$

$\begin{array}{lll}8.4 & \text { The Molecular Basis of Pluripotency } & 108\end{array}$

8.5 Cellular Reprogramming: Altering the Epigenetic State 110

8.6 Cellular Reprogramming: Primary Regulatory Pathways 111

$\begin{array}{ll}\text { 8.6.1 Temporal and Stoichiometric Considerations } & 113\end{array}$

$\begin{array}{ll}\text { 8.6.2 Target Cell Type } & 113\end{array}$

$\begin{array}{lll}8.7 & \text { Reprogramming Methods } & 114\end{array}$

$\begin{array}{lll}\text { 8.7.1 Viral-Driven } & 114\end{array}$

$\begin{array}{ll}\text { 8.7.2 Nucleic Acid/Episomal-Driven } & 115\end{array}$

$\begin{array}{ll}\text { 8.7.3 mRNA-Driven } & 117\end{array}$

$\begin{array}{ll}\text { 8.7.4 miRNA-Driven } & 117\end{array}$

$\begin{array}{lll}\text { 8.7.5 Protein-Driven } & 118\end{array}$

$\begin{array}{ll}\text { 8.7.6 External Factors/Enhancers } & 118\end{array}$

$\begin{array}{ll}\text { 8.7.7 Direct Reprogramming } & 120\end{array}$

$\begin{array}{lll}8.8 & \text { Applications and Future Trends } & 121\end{array}$

8.8.1 Moving Toward Clinical Applications for Cellular Reprogramming 121

8.8.2 The Merging of Stem Cells and New Methods of Genetic Engineering $\quad 125$

$\begin{array}{ll}\text { 8.8.3 Efficiency, Expense, and Safety } & 125\end{array}$

$\begin{array}{ll}\text { 8.8.4 Developing Standards } & 126\end{array}$

$\begin{array}{lll}8.9 \text { Conclusion } & 127\end{array}$

$\begin{array}{ll}\text { References } & 127\end{array}$

9 Chemicals Facilitating Reprogramming 141

Zhong-Dong Shi, Federico González, and Danwei Huangfu

$\begin{array}{lll}9.1 & \text { Introduction } & 141\end{array}$

9.2 Chemicals Modulating Epigenetic Barriers 145

9.2.1 Histone Deacetylase Inhibitors 146

9.2.2 Histone Methyltransferase Inhibitor and Demethylase

9.2.3 DNA Methyltransferase Inhibitors $\quad 149$

$\begin{array}{lll}9.3 & \text { Chemicals Targeting Signaling Pathways } & 150\end{array}$

$\begin{array}{ll}\text { 9.3.1 TGF } \beta \text { Signaling Inhibitors } & 150\end{array}$

9.3.2 Wnt Signaling and GSK3 Inhibitors 151

9.3.3 Other Kinase Inhibitors and Activators 152

9.3.4 Cell Senescence Alleviators 153

9.4 Chemicals Promoting Lineage Reprogramming 154

$\begin{array}{ll}9.5 \text { Conclusion } & 155\end{array}$

References 156 
10 Chemicals Facilitating Reprogramming: Targeting the SAM Binding Site to Identify Novel Methyltransferase Inhibitors

Jeong-Do Kim, Jong S. Rim, Robert B. Crochet, Yong-Hwan Lee, Jaroslaw Staszkiewicz, Ru Gao, and Kenneth J. Eilertsen

10.1 Introduction

163

10.2 DNA Methyltransferases, Inhibition, and Reprogramming 164

10.3 DNMT Inhibitors

164

10.4 Histone Methyltransferases, Inhibition, and Reprogramming 167

$\begin{array}{lll}10.5 & \text { Inhibitors of Lysine Methyltransferases } & 168\end{array}$

10.6 Identification of DNMT1 Inhibitor Candidates Using Virtual Screening 169 10.6.1 Functional Screening Using a DNMT1 Activity Assay 169

10.7 Targeting the SAM Binding Site to Identify Novel HMT Inhibitors 171 10.7.1 SAM Competitive Assay 173

10.7.2 SAM Binding Site is Unique and Selective across Multiple Epigenetic Targets 173

$\begin{array}{lll}10.8 \text { Conclusion } & 177\end{array}$

$\begin{array}{ll}\text { References } & 177\end{array}$

11 Biomaterials for Directed Differentiation $\quad 181$

Xintong Wang, Angela L. Zachman, Simon Maltais, and Hak-Joon Sung

$\begin{array}{llr}11.1 & \text { Introduction } & 182\end{array}$

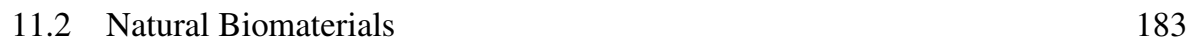

$\begin{array}{lll}\text { 11.2.1 } & \text { ECM-Derived Materials } & 183\end{array}$

$\begin{array}{lll}\text { 11.2.1.1 Matrigel } & 183\end{array}$

$\begin{array}{lll}11.2 .1 .2 & \text { Fibrin } & 184\end{array}$

$\begin{array}{lll}11.2 .1 .3 \text { Collagen } & 185\end{array}$

$\begin{array}{lll}\text { 11.2.1.4 Laminin } & 187\end{array}$

$\begin{array}{lll}\text { 11.2.2 Non-ECM-Derived Materials } & 188\end{array}$

$\begin{array}{ll}\text { 11.2.2.1 Chitosan } & 188\end{array}$

$\begin{array}{llr}11.3 & \text { Synthetic Biomaterials } & 189\end{array}$

$\begin{array}{lll}\text { 11.3.1 Polyesters } & 189\end{array}$

11.3.1.1 Poly(Lactic Acid) and Poly(Glycolic Acid)

Copolymers 189

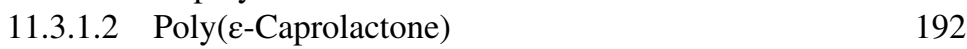

$\begin{array}{ll}\text { 11.3.2 Polyethylene Glycol } & 194\end{array}$

$\begin{array}{lll}11.4 & \text { Conclusion } & 195\end{array}$

$\begin{array}{lr}\text { References } & 196\end{array}$

12 Practicalities to Translation from the Clinic to the Market 203

Devyn M. Smith

$\begin{array}{lll}12.1 & \text { Introduction } & 203\end{array}$

12.2 Commercialization Comparison with Small Molecules, Medical Devices, and Biologics 
12.3 Historical Review and Case Studies 205

$\begin{array}{lll}\text { 12.3.1 Dermagraft } & 205\end{array}$

12.3.2 Provenge 206

12.4 Commercialization Challenges and How to Overcome Them 209

12.5 Translation from the Bench to the Clinic: Key Considerations 209

$\begin{array}{ll}12.6 \text { Conclusion } & 213\end{array}$

$\begin{array}{ll}\text { References } & 214\end{array}$

$\begin{array}{ll}\text { Index } & 217\end{array}$ 


\section{List of Contributors}

Dikshya Bastakoty, Department of Pathology, Vanderbilt University, USA

Maria Borowski, Department of Cell and Developmental Biology, University of Massachusetts Medical School, USA

Jeffery B. Bylund, Department of Medicine and Department of Pharmacology, Vanderbilt University, USA

Robert B. Crochet, Department of Biological Science, Louisiana State University, USA

Kenneth J. Eilertsen, NuPotential, Inc., USA and Pennington Biomedical Research Center, LSU System, USA

Bryan A. Fioret, Department of Medicine, Vanderbilt University, USA

Ru Gao, NuPotential, Inc., USA

Federico González, Developmental Biology Program, Memorial Sloan-Kettering Cancer Center, USA

Jijun Hao, College of Veterinary Medicine, Western University of Health Sciences, USA

Antonis K. Hatzopoulos, Department of Medicine, Vanderbilt University, USA

Charles C. Hong, Department of Medicine, Vanderbilt University, USA

Danwei Huangfu, Developmental Biology Program, Memorial Sloan-Kettering Cancer Center, USA

Daqing Jin, Department of Genetics, Fudan University School of Life Sciences, China

Jeong-Do Kim, NuPotential, Inc., USA

Joseph C. Laning, Research and Development, Provia Laboratories, LLC, USA

Yong-Hwan Lee, Department of Biological Science, Louisiana State University, USA 
Qiao Li, Department of Genetics, Fudan University School of Life Sciences, China

Simon Maltais, Department of Cardiac Surgery, Vanderbilt University, USA

Nikhil Munshi, Internal Medicine, University of Texas Southwestern Medical Center, USA

Young-Jae Nam, Department of Medicine, Division of Cardiovascular Medicine, Vanderbilt University, USA

David T. Paik, Department of Medicine, Vanderbilt University, USA

Jong S. Rim, NuPotential, Inc., USA

Sarika Saraswati, Department of Pathology, Microbiology and Immunology, Vanderbilt University School of Medicine, USA

Calvin C. Sheng, Department of Cardiovascular Medicine, Vanderbilt University, USA

Zhong-Dong Shi, Developmental Biology Program, Memorial Sloan-Kettering Cancer Center, USA

Devyn M. Smith, Neusentis Research Unit, Pfizer Worldwide R\&D, UK

Kelly P. Smith, Department of Cell and Developmental Biology, University of Massachusetts Medical School, USA

Jaroslaw Staszkiewicz, NuPotential, Inc., USA

Hak-Joon Sung, Department of Biomedical Engineering, School of Medicine, Vanderbilt University, USA

Xintong Wang, Department of Biomedical Engineering, Vanderbilt University, USA

Pampee P. Young, Department of Pathology, Microbiology and Immunology, Vanderbilt University, USA

Angela L. Zachman, Department of Biomedical Engineering, Vanderbilt University, USA

Tao P. Zhong, State Key Laboratory of Genetic Engineering, Fudan University School of Life Sciences, China; Department of Medicine, Vanderbilt University, USA 


\section{Preface}

The nascent field of regenerative medicine has advanced at a rapid pace, and clinical trials are ongoing to assess the safety and efficacy of stem cell-based therapies in indications that range from congestive heart failure to Crohn's disease. However, in general there is a lack of understanding of the specific mechanisms of action, and application of these new biologic products faces special challenges.

Four key areas in regenerative medicine are highlighted in this book: (i) cell-fate determination, (ii) regeneration, (iii) designer cells, and (iv) translation of cell-based therapies. The utility of stem cell-based therapies relies upon efficient manipulation of cell fates, not only to generate sufficient quantities of the desired cell type for transplantation but also to prevent tumor formation from residual, undifferentiated cells. The biologic mechanisms related to cell-fate determination will be presented in depth to provide the necessary background. While the focus of stem cell-based therapies has traditionally been on transplantation and engraftment, this is not the only paradigm. Instead, some therapies may stimulate regeneration in the host tissue through activation of endogenous stem-cell pools. In this volume, the use of mesenchymal stem cells (MSCs) in the context of wound healing and regeneration will be discussed. In addition, in recognition of the revolutionary advances in patient-derived induced pluripotent stem cells (iPSCs) and reprogramming of somatic cells, the issue of customizing iPSCs for research and clinical applications will be discussed. A myriad of technical and conceptual issues remain to be overcome before the full potential of iPSC technologies is realized, which will require an interdisciplinary approach. Thus, this book pays particular attention to the use of small molecules and biomaterials in addressing current challenges. Lastly, an overview of the practicalities related to translation of stem-cell therapies for clinical use will be provided.

This book is intended for those with interests ranging from basic developmental biology to stem-cell therapies. Biomedical researchers, clinicians, managers in biotechnology, and undergraduate and graduate students interested in stem cells and cell-based therapies will find this book useful in summarizing the most recent developments in stem-cell research.

The intrigue surrounding stem cell-based therapies lies not only in the treatment of chronic diseases but also in the potential to cure them. Therefore, regenerative medicine is rapidly moving to the forefront of many therapeutic areas, from cancer to heart disease. This book aims to summarize the collective knowledge of active researchers, illustrate current perspectives on the most pressing obstacles to clinical application, and serve as a guide to what lies ahead.

Charles C. Hong, MD, PhD Ada $\mathrm{S}$. Ao, $\mathrm{PhD}$ Jijun Hao, PhD 


\title{
1 \\ Wnt Signaling in Regulation of Stem Cells
}

\author{
David T. Paik and Antonis K. Hatzopoulos \\ Department of Medicine, Vanderbilt University, USA
}

\subsection{Overview of Wnt Signaling}

The Wnt signaling pathway is classically divided into so-called canonical and noncanonical branches based on the activation of specific intracellular components. Canonical Wnt signaling is activated when Wnt ligands bind to the Frizzled (Fzd) family of 7-transmembrane domain receptors and co-receptors, such as low-density lipoprotein receptor-related protein (LRP) 5/6, Ryk, and Ror2 [1-4]. This disrupts the formation of the $\beta$-catenin destruction complex, which consists of the scaffolding protein Axin, the Adenomatous polyposis coli (APC) protein, Dishevelled (Dsh), casein kinase I $\alpha$ (CK-I $\alpha)$, and glycogen synthase kinase-3Inase (CK). The dissociation of the destruction complex leads to stabilization of cytoplasmic iationein, which translocates to the nucleus to interact with TCF/LEF transcription factors and initiate transcription of canonical Wnt signaling target genes, such as $c-M y c$, Axin2, and Snail [5-7]. When canonical Wnt signaling is turned off, the destruction complex phosphorylates $\beta$-catenin for ubiquitin-mediated proteosomal degradation [8].

Noncanonical Wnt signaling pathways are $\beta$-catenin-independent and are mediated through other intracellular proteins $[1-4,9,10]$. In the Wnt/JNK pathway, binding of Wnt to Fzd receptors activates small-GTPases, RhoA, and Rac through recruitment of Dsh, which thereby activate Rho kinase and c-Jun N-terminal kinases (JNK). In the Wnt/Ca ${ }^{+2}$ pathway, binding of Wnt to Fzd receptors increases intracellular $\mathrm{Ca}^{2+}$ levels, activating

Chemical Biology in Regenerative Medicine: Bridging Stem Cells and Future Therapies, First Edition. Edited by Charles C. Hong, Ada S. Ao, and Jijun Hao.

(C) 2014 John Wiley \& Sons, Ltd. Published 2014 by John Wiley \& Sons, Ltd. 
calcium/calmodulin-dependent kinase (CaMK) II, protein kinase $\mathrm{C}$ (PKC), and the protein phosphatase calcineurin $(\mathrm{CaCN})$ to trigger dephosphorylation of NF-AT transcription factors. Thus activated NF-AT transcription factors translocate to the nucleus to stimulate transcription of their target genes [11]. Intriguingly, noncanonical Wnt signaling has been shown to inhibit canonical Wnt signaling in various mechanisms [10, 12, 13].

To date, $19 \mathrm{Wnt}$ ligands and $10 \mathrm{Fzd}$ receptors have been identified. The $19 \mathrm{Wnt}$ genes fall into 12 conserved Wnt subfamilies, which exist in most mammalian genomes, including the human genome [14]. Different combinations of individual Wnt ligands, receptors, and co-receptors allow differential activation of $\beta$-catenin-dependent/canonical Wnt signaling, $\beta \mathrm{Wnt}$ signaling, ligands, receptors, and/or Wnt signaling in a cellular context-dependent manner.

In the past 2 decades, the connection between Wnt signaling and human disease has been well established. Numerous components of the pathway have been implicated in cancer, obesity, osteoporosis, diabetes, and cardiovascular diseases [15]. Interestingly, Wnt signaling components are also critical regulators of stem and progenitor cells in various organs and tissues. A thorough understanding of the Wnt signaling pathway in the regulation of stem cells will be instrumental in translating the potential of stem cells to effective therapeutic solutions for human degenerative diseases or to the restoration of organ function after injury.

\subsection{Wnt Signaling in Embryonic Stem Cells}

Wnt signaling has been implicated in the maintenance of the pluripotency and differentiation potential of embryonic stem cells (ESCs). A number of studies have demonstrated that individual Wnt ligands can stimulate self-renewal of ESCs [16-18]. Activation of the canonical Wnt pathway complements the LIF/JAK-STAT pathway via upregulation of the Stat3 gene to inhibit ESC differentiation [16]. Paracrine and autocrine Wnt signaling is essential not only for self-renewal of mESCs but also to inhibit differentiation into epiblast stem cells (epiSCs) [7]. Accordingly, mutations in the $\beta \mathrm{n}$ of the io destruction complex APC protein increase $\beta$-catenin levels, diminishing the differentiation capacity of mouse ESCs (mESCs) into the three germ layers [19].

In contrast, whether Wnt signaling promotes self-renewal or differentiation of human ESCs (hESCs) has been rather controversial. In the presence of supportive feeder cells or a conditioned medium $(\mathrm{CM})$ rich in factors preventing differentiation, Wnt3a enhanced selfrenewing proliferation of undifferentiated hESC $\mathrm{H} 1$ cells. In the absence of $\mathrm{CM}$, however, activation of Wnt signaling accelerated both proliferation and differentiation of hESCs [20]. The canonical Wnt ligand Wnt1 displayed the same effects on hESCs as Wnt3a, while the noncanonical Wnt ligand Wnt5a did not affect the proliferation of hESCs, indicating that $\beta$ catenin-dependent canonical Wnt activation is responsible for enhanced $\mathrm{hESC}$ proliferation $[21,22]$. Activation of the canonical Wnt pathway by 6-bromoindirubin- $3^{\prime}$-oxime (BIO), a specific inhibitor of glycogen synthase kinase 3 (GSK3), produced comparable results in maintaining the undifferentiated phenotype of hESCs marked by sustained expression of the pluripotent transcription factors OCT3/4 (POU5F1), REX1, and NANOG and by prevention of the epithelial-mesenchymal transition (EMT) of hESCs [23, 24]. In complementary fashion, addition of the Wnt inhibitors Sfrp-1, Sfrp-2, and Sfrp-4, singly or in combination, promoted differentiation of hESCs [22]. 
However, other studies have reported that canonical Wnt activation disrupted hESC self-renewal and promoted differentiation. Specifically, conditional activation of stabilized $\beta$-catenin in KhES-1 and KhES-3 lines resulted in downregulation of the pluripotent markers NANOG, SOX2, and POU5F1 and upregulation of the mesodermal marker T Brachyury, followed by induction of the ventral mesodermal and endothelial marker KDR (VEGFR2) and the early cardiac marker NKX2.5 [25].

Experimental evidence also suggests that canonical Wnt signaling interacts with Activin/Nodal and bone morphogenetic protein (BMP) signaling pathways to specify differentiation lineages in hESCs. Canonical Wnt activation induced Activin/Nodal and BMP signaling to promote posterior Primitive Streak (PS) and mesoderm differentiation of hESCs. Synergistic interaction between Wnt and Activin/Nodal pathways was shown to be required for anterior PS and endoderm specification, while BMP and MAPK signaling antagonized it [25]. Recent studies have also shown that hESCs treated with the Wnt inhibitor IWP are maintained as pluripotent, with the ability to differentiate into neural cells [26]. Furthermore, Wnt3a-treated hESCs acquired PS-like characteristics and differentiated into mesodermal and endodermal cells [26].

It is noteworthy that the effects of Wnt3a on hESCs and mESCs differ significantly. In mESCs, Wnt3a prevented progression of mESCs to EpiSCs, while in hESCs Wnt3a facilitated their differentiation into mesodermal and endodermal lineages [7, 26]. In another study, the pluripotent marker OCT4 in hESCs was shown to repress $\beta$-catenin during self-renewal, whereas knockdown of OCT4 activated canonical Wnt signaling [27]. In support of these studies, Wnt1-treated hESCs displayed induced differentiation to hemogenic endothelial cells, while treatment with the Wnt inhibitor Dkk1 reduced this differentiation potential [28].

The apparently contradictory reports of Wnt signaling in hESC self-renewal and differentiation may be due to the epiblast origin of the various hESC lines tested [25]. It has also been proposed that the effects of Wnt signaling on hESCs are highly sensitive to the level of Wnt activation [26,27]. Therefore, it is likely that apparently contradictory results regarding the role of Wnt signaling in human ESC pluripotency, proliferation, and differentiation reflect the heterogeneity of the corresponding lines and their sensitivity to canonical Wnt signaling levels.

\subsection{Wnt Signaling in Cardiovascular Progenitor Cells and Cardiomyocyte Differentiation}

Wnt signaling also plays important roles in the maintenance and expansion of cardiovascular progenitor cells and in their differentiation into endothelial and cardiomyocyte lineages $[2,10,11]$. The mammalian heart is one of the first organs to form during embryogenesis and Wnt signaling has been implicated in all phases of cardiogenesis. Initially, canonical Wnt signaling is necessary for the formation of mesodermal progenitor cells, but it must then be suppressed in order for mesoderm progenitors to yield cardiac progenitor cells (CPCs) [11]. Subsequently, noncanonical Wnt signaling is necessary for the specification of CPCs. Wnt5a, a noncanonical Wnt activator, is upregulated by the mesoderm-specific transcription factor Mesp-1 to promote formation of CPCs [29]. Other studies have shown that intrinsic Wnt2 expression in mouse ES cells is essential for efficient cardiomyocyte 
differentiation and that exogenous Wnt2 promotes cardiomyocyte differentiation. Interestingly, Wnt2 induced cardiogenesis through activation of the noncanonical JNK/AP-1 pathway [30]. After CPC specification, canonical Wnt signaling activation stimulates proliferation of Is11 ${ }^{+}$CPCs, whereas subsequent Dkk1 inhibition of canonical Wnt signaling and noncanonical Wnt activation by Wnt11 are required for cardiomyocyte differentiation [31,32].

The role of Wnt signaling and the effects of Wnt signaling manipulation during heart development have been well documented in various animal models. In mouse embryos, deletion of $\beta$-catenin in the definitive endoderm led to formation of multiple hearts along the anterior-posterior (A/P) axis, as one of the earliest pieces of evidence to implicate Wnt signaling in the endoderm in the induction of precardiac mesoderm [33]. Notably, the supernumerary hearts followed the ectopic expression patterns of BMP2. Ectopic cardiac tissue also formed in zebrafish embryos with overexpression of the BMP antagonist Gremlin2, while Gremlin2 morphants exhibited a rise in the intracellular levels of $\beta$-catenin, suggesting a crosstalk between Wnt and BMP signaling during cardiac development $[33,34]$. In chick embryos, Crescent, a Frizzled-related protein that inhibits Wnt8c, is expressed in the anterior endoderm during gastrulation, while Wnt3a and Wnt8c expression is localized to the primitive streak and posterior lateral plate. Dkk1 induces cardiac gene expression in the posterior lateral plate mesoderm, while ectopic Wnt activation in the anterior mesoderm inhibits cardiac formation. Therefore, inhibition of Wnt signaling in chick embryos promoted heart formation in the anterior lateral mesoderm, supporting a model that cardiogenesis initially requires high levels of BMP but low canonical Wnt activity [35]. In Xenopus embryos, Wnt6 was found to be expressed in tissues close to and inside the developing heart and to be required to restrict heart development, since the absence of Wnt6 led to an abnormally large heart [36]. Intriguingly, Wnt6 is not required during the gastrulation stage but rather in the later stages of organogenesis that precede the differentiation of cardiogenic mesoderm into myocardium. Overexpression of Wnt6 reflected such a phenotype through the activation of $\beta$-catenin-dependent/canonical Wnt signaling, which repressed cardiogenic transcription factors such as Gata6 and Mlc2. Sfrp-1, an endogenous Wnt inhibitor, is strongly induced in differentiating cardiomyocytes and participates in a negative-feedback regulatory loop of Wnt signaling in regulating the cell fate of cardiac mesoderm [37].

Later studies reinforced a biphasic role for canonical Wnt signaling in cardiac specification in zebrafish and mice. For example, it appears that $\beta$-catenin-dependent Wnt signaling before gastrulation promotes cardiac differentiation while inhibiting heart formation during gastrulation [38]. Early treatment of mESCs with Wnt3a induced mesoderm specification, which activated a feedback loop that subsequently repressed the Wnt pathway, which in turn increased cardiac differentiation. Late activation of $\beta$-catenin in mESCs, on the other hand, repressed cardiac differentiation. Overexpression of the noncanonical Wnt ligand Wnt11, which may inhibit canonical Wnt signaling, promoted cardiac differentiation during early stages but repressed it in later ones. Moreover, canonical Wnt signaling in the early stages of embryoid body (EB) formation induced cardiac differentiation but suppressed hematopoietic and vascular cell lineages. Activation of canonical Wnt signaling in the later stages of EB formation, however, inhibited cardiac formation and induced expression of hematopoietic and vascular genes through the suppression of BMP signaling [39]. Such results highlight the strict temporal and spatial requirement of Wnt signaling 
in heart development. Current evidence suggests that $\beta$-catenin-dependent Wnt signaling promotes proliferation of mesodermal and cardiovascular progenitors but that noncanonical Wnt signaling promotes differentiation of mesodermal stem cells to CPCs and of CPCs to cardiomyocytes.

Based on the finding that Wnt signaling plays a critical role in cardiomyogenesis during development, recent studies have identified small-molecule compounds that modulate Wnt signaling, thus regulating cardiomyogenesis. Using in vivo zebrafish embryo screening, a small molecule that inhibits canonical Wnt signaling called cardionogen was shown to induce cardiogenesis during and after gastrulation but to prevent heart formation before gastrulation [40]. XAV939, a small-molecule inhibitor of canonical Wnt signaling, robustly induced cardiomyogenesis in mESCs when administered immediately following the formation of mesoderm progenitor cells [41]. Similarly, KY0211 is another example of a small molecule that promotes differentiation of human pluripotent stem cells (hPSCs) into cardiomyocytes by inhibiting Wnt signaling [42]. Small molecules inhibiting canonical Wnt signaling robustly drove the expression of red fluorescent reporter protein driven by the cardiac-specific MYH6 promoter in hESCs [43]. It was found that Wnt inhibition specifically promoted cardiomyocyte differentiation from hESC-derived mesodermal cells, without inducing other mesodermal derivatives such as endothelial cells or smooth muscle cells. Other known canonical Wnt inhibitors, such as IWR1, IWP3, and XAV939, reproduced the same phenotype, supporting the connection between timely inhibition of Wnt signaling and cardiac cell differentiation from hESCs. Such findings were reported in human induced pluripotent stem cells (hiPSCs) as well. Early treatment of hiPSCs with BMP4 followed by late treatment with small-molecule Wnt inhibitors such as IWR1 resulted in a marked increase in cardiomyocyte differentiation [44]. In summary, various small-molecule canonical Wnt inhibitors were shown to promote cardiomyocyte differentiation from several stem cell lines, including mouse and human ESCs and hiPSCs. It will be interesting to test the effect of these molecular tools on adult cardiovascular progenitor cells and cardiac tissue regeneration. So far, the canonical Wnt signaling inhibitor pyrvinium has been shown to promote wound repair and post-myocardial infarction (MI) cardiac remodeling [45].

\subsection{Wnt Signaling in Mesenchymal Stem Cells}

Mesenchymal stem cells (MSCs) have attracted considerable interest as a promising therapeutic tool for various human diseases due to their plasticity and potential for tri-lineage differentiation into osteoblasts, adipocytes, and chondrocytes [46]. Wnt signaling has a key function in the cell fate decisions of multipotent MSCs. Specifically, the canonical Wnt activator Wnt10b promotes osteoblastogenesis at the expense of adipogenesis $[47,48]$. Wnt10b enables this switch in MSC fate by inducing the osteoblastogenic transcription factors Runx2, Dlx5, and Osterix and suppressing the adipogenic transcription factors PPAR $\gamma$ and $\mathrm{C} / \mathrm{EBP} \alpha[47,49,50]$. As a result, FABP4-Wnt10b transgenic mice that overexpress the Wnt10b gene in their bone marrow exhibited increased bone mass, density, and strength, while $\mathrm{Wnt} 10 \mathrm{~b}^{-1-}$ mice had decreased trabecular bone and serum osteocalcin [47]. At the same time, FABP4-Wnt10b mice, which express Wnt10b in their white and brown adipose tissue, resisted accumulation of adipose tissue when fed a high-fat diet [51]. These results 
provide compelling evidence that Wnt10b is a cell fate regulator of MSCs, influencing the physiology of bone and adipose tissue, and serves as a potential therapeutic target for diseases such as osteoporosis and obesity.

Similarly, canonical Wnt signaling has been shown to promote osteoblastogenesis in human amniotic fluid mesenchymal stem cells (huAFMSCs). Specifically, canonical Wnt signaling activation was linked to osteogenic differentiation of huAFMSCs through induction of Dishevelled-2 (Dvl-2), and increased levels of active $\beta$-catenin and phosphorylated GSK3 $\beta$. Conversely, inhibition of Wnt signaling by Dkk-1 led to suppression of the osteogenic transcription factor Runx2 and activation of FABP4, favoring adipocyte differentiation [52]. Moreover, studies have identified Wnt6, Wnt10a, and Wnt10b as inhibitors of adipogenesis. Notably, the three canonical Wnt ligands affect MSC differentiation to varying degrees. While Wnt10a affects MSC differentiation to a similar extent as Wnt10b, knockdown of endogenous Wnt6 stimulates preadipocyte differentiation and impairs osteoblastogenesis to greater degrees than that of Wnt10a or Wnt10b, suggesting that Wnt6 is a more potent endogenous regulator of MSC fate than are the other Wnt factors [50]. The effects of Wnt6, Wnt10a, and Wnt10b on MSC differentiation are $\beta$-catenin-dependent, as knockdown of $\beta$-catenin prevents the inhibition of adipogenesis and stimulation of osteoblastogenesis by the three Wnt ligands [50].

Interestingly, mutations in the human $W N T 10 B$ gene have been found in obesity patients in various hereditary groups. Six single-nucleotide polymorphisms (SNPs) of WNT10B were identified from direct sequencing of the genomic DNA of 1029 obese Korean female subjects and four were found from 1013 obese Belgian male subjects, while a C256Y mutation in WNT10B was found in 115 obese Italian male subjects [53-55]. Although the exact molecular mechanisms connecting Wnt10b to familial obesity remain unknown, AP-1 and NF- $\kappa \mathrm{B}$ binding sites in the promoter areas are conserved among mammalian $W N T 10 B$ orthologs [56]. This finding could explain the upregulation of WNT10B by tumor necrosis factor (TNF) $\alpha$, as TNF $\alpha$ signaling via TNFR1 and TRADD/RIP/TRAF2 complex activates JNK and NF- $\mathrm{KB}$. It is likely that increased Wnt10b levels block adipogenesis in favor of osteogenic differentiation of MSCs, whereas defective Wnt10B activity leads to obesity [56,57]. It is likely that defective Wnt activity impairs these negative-feedback mechanisms, enhancing adipogenic differentiation of MSCs.

An additional connection has been described between NF- $\kappa \mathrm{B}$ and Wnt signaling pathways in the differentiation of MSCs [58]. It was found that the proinflammatory cytokines TNF and IL-17 activated the IKK/NF- $\mathrm{B}$ pathway to inhibit osteogenic differentiation of MSCs. Inhibition of IKK/NF- $\kappa \mathrm{B}$, on the other hand, promoted bone formation by suppressing $\beta$-catenin ubiquitination and degradation. Furthermore, Wnt 4 , a noncanonical Wnt ligand, also enhanced in vitro osteogenic differentiation of MSCs from human craniofacial tissues and promoted bone formation in vivo [59]. Wnt4 did not stabilize $\beta$-catenin but did activate p38 MAPK in an Axin-dependent manner. Intriguingly, Wnt4-overexpressing MSCs improved the healing of craniofacial defects in a murine model, indicating that both canonical and noncanonical Wnt signaling can promote osteogenic differentiation via seemingly independent signaling cascades. In contrast, other investigators reported that activation of $\beta$-catenin-dependent Wnt signaling suppressed the noncanonical Wnt/Ca ${ }^{+2}$ pathway, leading to increased proliferation but attenuated osteogenic differentiation of periodontal ligament stem cells (PDLSCs), a specific subpopulation of MSCs [60]. Treatment 
with Dkk1 rescued the $\mathrm{Wnt} / \mathrm{Ca}^{+2}$ signaling pathway, leading to osteogenic differentiation of PDLSCs. Taken together, these studies implicate Wnt signaling in the regulation of MSC differentiation. However, seemingly contradictory results suggest that this regulation is complex, due to feedback regulatory loops and context-dependent cellular responses to Wnt signaling.

Inhibition of Wnt signaling may also increase the therapeutic potential of MSCs. The Wnt antagonist Sfrp-2 enhanced MSC engraftment and survival, where Sfrp-2-overexpressing MSCs were shown to be more effective in wound and myocardial repair [61]. The observed Sfrp-2 effects were due to dual inhibition of Wnt and BMP signaling pathways [62]. Similarly, Akt-overexpressing MSCs (Akt-MSCs) induced high Sfrp-2 expression and promoted cardiomyocyte cell survival through an Sfrp-2-mediated reduction in caspase-3 activity $[63,64]$. The small-molecule Wnt inhibitor pyrvinium recapitulated the Sfrp2 effects on MSCs [45]. These results indicate that activation of Wnt signaling leads to senescence of MSCs, while inhibition of Wnt signaling promotes self-renewal and engraftment of MSCs [62]. Therefore, manipulation of Wnt signaling in MSCs may prove beneficial in clinical trials that transplant MSCs to injured tissues with limited regenerative capacity, such as in ischemic myocardium after acute myocardial infarction (MI).

\subsection{Wnt Signaling in Hematopoiesis and Hematopoietic Stem Cells}

Wnt signaling is essential for hematopoiesis and differentiation of hematopoietic stem cells (HSCs) [65, 66]. Various gain-of-function and loss-of-function approaches have provided insights into the role of Wnt signaling in HSC differentiation [67]. For example, constitutively active $\beta$-catenin in Bcl2-transgenic hematopoietic stem/progenitor cells led to proliferation of HSCs via induction of HoxB4 and Notch1. In parallel, overexpression of stabilized $\beta$-catenin in mice inhibited multilineage differentiation and resulted in transient expansion of HSCs [68-70]. In agreement with these studies, deletion of $\beta$ In agnin in HSCs reduced their self-renewing capacity [71], while the canonical Wnt activator Wnt3a promoted reacquisition of stem cell-like characteristics in committed lymphoid progenitor cells [72].

Although these studies underline the importance of canonical Wnt signaling in HSC proliferation and prevention of differentiation, approaches using additional Wnt ligands point to a more complex picture. For instance, the noncanonical Wnt ligand Wnt 4 was also shown to increase proliferation of hematopoietic progenitors and another noncanonical Wnt ligand, Wnt5a, promoted HSC renewal by antagonizing canonical Wnt signaling in HSCs $[73,74]$. In zebrafish, the noncanonical Wnt activator Wnt16 was identified as interacting with Notch signaling in specifying hematopoietic stem cells [65]. Wnt16 is required for somatic expression of the Notch ligands deltaC (dlc) and deltaD (dld), essential factors in definitive hematopoiesis. Through the use of a Wnt-reporter mouse, canonical Wnt signaling was shown to regulate hematopoiesis in a dosage-dependent manner, where lineage-specific Wnt dosages regulated HSCs, myeloid precursors, and T lymphoid precursors during hematopoiesis [75]. These seemingly contradictory findings from gainof-function and loss-of-function experiments may be credited to different levels of Wnt signaling expressed in individual experimental model systems. However, it is conceivable 
that controlled regulation of Wnt signaling might provide an effective solution for leukemia treatment [76].

\subsection{Wnt Signaling in Neural Stem Cells}

Various neural cell types, such as neurons, astrocytes, and oligodendrocytes, are derived from a single neural progenitor cell [77]. In adult neurogenesis, neural stem cells (NSCs) are found in the subventricular zone of the lateral ventricles and the subgranular zone of the hippocampal dentate gyrus, and evidence suggests that Wnt signaling is associated with the regulation of both populations [3]. For example, Wnt3a has been shown to locally regulate the expansion of the caudomedial cortex, the site of hippocampal development in the mouse brain [78]. Wnt3a loss of function resulted in limited proliferation of caudomedial cortical progenitor cells, indicating that canonical Wnt activation by Wnt3a is necessary for the development and growth of the hippocampus. An independent study confirmed this notion by showing that canonical Wnt signaling activation via Wnt3a overexpression increased neurogenesis from adult hippocampal stem/progenitor cells both in vivo and in vitro; in contrast, ablation of Wnt signaling blocked neurogenesis [79]. Furthermore, stabilized $\beta$-catenin in neural precursors resulted in enlarged brains with an increased cerebral cortical area in mice [80]. More recently, activation of canonical Wnt signaling selectively stimulated proliferation within the oligodendrogliogenic lineage, but not the neurogenic lineage, in adult NSCs in the mouse subependymal zone [74]. These findings underscore the role of Wnt signaling in regulating cell fate decisions in NSCs, echoing observations in other types of multipotent stem cell.

\subsection{Wnt Signaling in Endothelial-Mesenchymal Transition}

Aside from embryonic development, stem and progenitor cells are essential for maintaining homeostasis in virtually all adult organs and tissues. One common feature of stem and progenitor cells is their location in perivascular areas. Blood vessels, composed of endothelial cells and mural cells, are typically associated with stem cell niches that protect and regulate both vascular and nonvascular stem and progenitor cells [81]. Thus, blood vessels provide a niche for organ-specific stem cells [81].

Furthermore, there is mounting evidence that endothelial cells in adult tissues can transition into mesenchymal cell types under injury or stress conditions, a phenomenon known as endothelial-mesenchymal transition (EndMT), acting as a source of progenitor cells and of cells involved in tissue repair [82-86]. EndMT was originally described in embryonic heart formation, with endocardial cells in the cardiac cushions undergoing EndMT to form the cardiac valves $[11,87]$. On the other hand, in the adult heart, EndMT appears to give rise to myofibroblasts, thereby contributing to pathological fibrosis $[84,88]$. In the adult mouse heart, following myocardial infarction (MI), approximately $30 \%$ of myofibroblasts formed in the infarct tissue arise from endothelial cells [88].

The EndMT during cardiac fibrosis was shown to be mediated by transforming growth factor beta-1 (TGF $\beta 1$ ) signaling in a Smad-dependent manner and could be repressed by treatment with BMP7 [84]. Further studies revealed that mutations in ALK2 and 
treatment with TGF $\beta 2$ or BMP4 can also lead to EndMT, in which the endothelial-derived mesenchymal cells exhibit the potential to differentiate into chondrocytes and osteoblasts, much like MSCs [83]. In addition, EndMT has been shown to contribute to perivascular mural cells, such as smooth muscle cells and pericytes [89,90], and to approximately $40 \%$ of cancer-associated fibroblasts (CAFs) in the tumor stroma [85].

Wnt signaling has been implicated as a regulator of EndMT as well. EndMT-derived repair cells were found to be canonical Wnt-activated, possibly by the MI-induced Wnt ligands Wnt2 and Wnt10b [88]. In the homeostatic heart, canonical $\mathrm{Wnt}^{+}$cells were localized to vascular smooth muscle cells of the aorta and periepicardial blood vessels but not to other cell types. Upon injury, however, large numbers of endothelial cells became canonical Wnt-activated and transitioned into $\mathrm{CD} 31^{+} / \alpha \mathrm{SMA}^{+}$progenitor cells, which gave rise to myofibroblasts. Supporting the role of canonical Wnt signaling in this process, the Wnt activator BIO induced EndMT in bEnd-3 endothelial cells in vitro [88].

Another study showed however that Dkk1-mediated inhibition of Wnt signaling promoted EndMT marked by induction of myofibroblasts and osteogenic markers and inhibited cobblestone morphology and capillary-like tube formation of endothelial cells [91]. Dkk1 was shown to promote Alk-mediated Smad activation, which is responsible for triggering the EndMT and osteogenic differentiation of endothelial cells. In a complementary fashion, Wnt activation by Wnt7b maintained the endothelial phenotype and prevented EndMT [91]. Consistent with this finding, persistent expression of the Wnt receptor Leucine-rich repeatcontaining G protein-coupled receptor 5 (LGR5) inhibited EndMT in corneal endothelial cells [92]. R-spondin-1, a LGR5 ligand, similarly inhibited EndMT and promoted endothelial cell proliferation [92].

The discrepancies found in the role of Wnt signaling in EndMT are likely due to the fact that various Wnt activators and inhibitors target different nodes of the signaling pathway. For example, the Wnt chemical activator BIO targets GSK3 $\beta$, an intercellular member of the destruction complex of $\beta$-catenin, while Dkk1 and Wnt7b are extracellular proteins that interact with various cell-membrane receptors such as Frizzled, LRP5/6, LGR5, and Alk receptors [91]. It is likely that unlike chemical compounds, proteins such as Dkk1 trigger a complex crosstalk of negative and positive feedback regulatory loops between various Wnt signaling branches, as well as other pathways such as Alk/BMP/Smad, complicating straightforward interpretation of experimental results.

\subsection{Conclusion}

Wnt signaling is an essential developmental pathway. As discussed in this chapter, studies have identified it to be required for the maintenance, self-renewal, and differentiation of various types of stem and progenitor cell, as a major regulator of stemness and stem-cellfate decisions. In addition to the cases of adult stem cells described here, Wnt signaling has also been extensively studied in the biology of stem cells in gut epithelium and hair follicles [15, 93, 94]. In general, activation of canonical Wnt signaling is typically required for the proliferation and self-renewal of multi- or pluripotent stem and progenitor cells, while inhibition of canonical Wnt or activation of noncanonical Wnt signaling promotes differentiation of stem and progenitor cells. It is also noteworthy that Wnt signaling effects are complex and stage-specific, requiring the development of elaborate protocols to 
stimulate and inhibit various Wnt signaling branches in a timely fashion. The significance of controlled Wnt signaling in the stem cell maintenance is also reflected by the fact that deregulation of the Wnt pathway is linked to many forms of cancer. Currently, a large number of reagents are being developed to inhibit or stimulate Wnt signaling. Such tools may revolutionize Wnt signaling regulation for the expansion and differentiation of organspecific stem cells in the treatment of human degenerative diseases or for the regeneration of tissue after injury.

\section{References}

(1) Van Amerongen, R. and Nusse, R. (2009) Towards an integrated view of Wnt signaling in development. Development (Cambridge England), 136 (19), 3205-3214.

(2) Rao, T.P. and Kühl, M. (2010) An updated overview on Wnt signaling pathways: a prelude for more. Circulation Research, 106 (12), 1798-1806.

(3) Nusse, R. (2008) Wnt signaling and stem cell control. Cell Research, 18 (5), 523-527.

(4) MacDonald, B.T., Tamai, K., and He, X. (2009) Wnt/beta-catenin signaling: components, mechanisms, and diseases. Developmental Cell, 17 (1), 9-26.

(5) Jho, E., Zhang, T., Domon, C. et al. (2002) Wnt/beta-catenin/Tcf signaling induces the transcription of Axin2, a negative regulator of the signaling pathway. Molecular and Cellular Biology, 22 (4), 1172-1183.

(6) He, T.C., Sparks, A.B., Rago, C. et al. (1998) Identification of c-MYC as a target of the APC pathway. Science, 281 (5382), 1509-1512.

(7) Ten Berge, D., Kurek, D., Blauwkamp, T. et al. (2011) Embryonic stem cells require Wnt proteins to prevent differentiation to epiblast stem cells. Nature Cell Biology, 13 (9), 1070 1075.

(8) Liu, C., Li, Y., Semenov, M. et al. (2002) Control of beta-catenin phosphorylation/degradation by a dual-kinase mechanism. Cell, 108 (6), 837-847.

(9) Veeman, M.T., Axelrod, J.D., and Moon, R.T. (2003) A second canon: functions and mechanisms of $\beta$-catenin-independent Wnt signaling. Developmental Cell, 5 (3), 367-377.

(10) Cohen, E.D., Tian, Y., and Morrisey, E.E. (2008) Wnt signaling: an essential regulator of cardiovascular differentiation, morphogenesis and progenitor self-renewal. Development, 135 (5), 789-798.

(11) Gessert, S. and Kühl, M. (2010) The multiple phases and faces of Wnt signaling during cardiac differentiation and development. Circulation Research, 107 (2), 186-199.

(12) Pandur, P., Läsche, M., Eisenberg, L.M., and Kühl, M. (2002) Wnt-11 activation of a non-canonical Wnt signalling pathway is required for cardiogenesis. Nature, 418 (6898), 636-641.

(13) Maye, P., Zheng, J., Li, L., and Wu, D. (2004) Multiple mechanisms for Wnt11-mediated repression of the canonical Wnt signaling pathway. Journal of Biological Chemistry, 279 (23), 24659-24665.

(14) Kusserow, A., Pang, K., Sturm, C. et al. (2005) Unexpected complexity of the Wnt gene family in a sea anemone. Nature, 433 (7022), 156-160.

(15) Clevers, H. and Nusse, R. (2012) Wnt/ $\beta$-catenin signaling and disease. Cell, 149 (6), 1192-1205.

(16) Hao, J., Li, T.-G., Qi, X. et al. (2006) WNT/ $\beta$-catenin pathway up-regulates Stat 3 and converges on LIF to prevent differentiation of mouse embryonic stem cells. Developmental Biology, 290 (1), 81-91.

(17) Ogawa, K., Nishinakamura, R., Iwamatsu, Y. et al. (2006) Synergistic action of Wnt and LIF in maintaining pluripotency of mouse ES cells. Biochemical and Biophysical Research Communications, 343 (1), 159-166.

(18) Singla, D.K., Schneider, D.J., LeWinter, M.M., and Sobel, B.E. (2006) wnt3a but not wnt11 supports self-renewal of embryonic stem cells. Biochemical and Biophysical Research Communications, 345 (2), 789-795. 
(19) Kielman, M.F., Rindapää, M., Gaspar, C. et al. (2002) Apc modulates embryonic stem-cell differentiation by controlling the dosage of $\beta$-catenin signaling. Nature Genetics, 32 (4), 594 605.

(20) Dravid, G., Ye, Z., Hammond, H. et al. (2005) Defining the role of Wnt/ $\beta$-catenin signaling in the survival, proliferation, and self-renewal of human embryonic stem cells. Stem Cells, $\mathbf{2 3}$ (10), 1489-1501.

(21) Cai, L., Ye, Z., Zhou, B.Y. et al. (2007) Promoting human embryonic stem cell renewal or differentiation by modulating Wnt signal and culture conditions. Cell Research, 17 (1), 62-72.

(22) Villa-Diaz, L.G., Pacut, C., Slawny, N.A. et al. (2009) Analysis of the factors that limit the ability of feeder cells to maintain the undifferentiated state of human embryonic stem cells. Stem Cells and Development, 18 (4), 641-651.

(23) Sato, N., Meijer, L., Skaltsounis, L. et al. (2004) Maintenance of pluripotency in human and mouse embryonic stem cells through activation of Wnt signaling by a pharmacological GSK3-specific inhibitor. Nature Medicine, 10 (1), 55-63.

(24) Ullmann, U., Gilles, C., Rycke, M.D. et al. (2008) GSK-3-specific inhibitor-supplemented hESC medium prevents the epithelial-mesenchymal transition process and the up-regulation of matrix metalloproteinases in hESCs cultured in feeder-free conditions. Molecular Human Reproduction, 14 (3), 169-179.

(25) Sumi, T., Tsuneyoshi, N., Nakatsuji, N., and Suemori, H. (2008) Defining early lineage specification of human embryonic stem cells by the orchestrated balance of canonical Wnt/ $\beta$-catenin, Activin/Nodal and BMP signaling. Development, 135 (17), 2969-2979.

(26) Blauwkamp, T.A., Nigam, S., Ardehali, R. et al. (2012) Endogenous Wnt signalling in human embryonic stem cells generates an equilibrium of distinct lineage-specified progenitors. Nature Communications, 3, 1070.

(27) Davidson, K.C., Adams, A.M., Goodson, J.M. et al. (2012) Wnt/ $\beta$-catenin signaling promotes differentiation, not self-renewal, of human embryonic stem cells and is repressed by Oct 4 . Proceedings of the National Academy of Sciences of the United States of America, 109, 44854499.

(28) Woll, P.S., Morris, J.K., Painschab, M.S. et al. (2008) Wnt signaling promotes hematoendothelial cell development from human embryonic stem cells. Blood, 111 (1), 122-131.

(29) Chen, V.C., Stull, R., Joo, D. et al. (2008) Notch signaling respecifies the hemangioblast to a cardiac fate. Nature Biotechnology, 26 (10), 1169-1178.

(30) Onizuka, T., Yuasa, S., Kusumoto, D. et al. (2012) Wnt2 accelerates cardiac myocyte differentiation from ES-cell derived mesodermal cells via non-canonical pathway. Journal of Molecular and Cellular Cardiology, 52 (3), 650-659.

(31) Qyang, Y., Martin-Puig, S., Chiravuri, M. et al. (2007) The renewal and differentiation of Isl1+ cardiovascular progenitors are controlled by a Wnt/beta-catenin pathway. Cell Stem Cell, 1 (2), 165-179.

(32) Rai, M., Walthall, J.M., Hu, J., and Hatzopoulos, A.K. (2012) Continuous antagonism by Dkk1 counter activates canonical Wnt signaling and promotes cardiomyocyte differentiation of embryonic stem cells. Stem Cells and Development, 21 (1), 54-66.

(33) Lickert, H., Kutsch, S., Kanzler, B. et al. (2002) Formation of multiple hearts in mice following deletion of beta-catenin in the embryonic endoderm. Developmental Cell, 3 (2), 171-181.

(34) Müller, I.I., Melville, D.B., Tanwar, V. et al. (2012) Functional modeling in zebrafish demonstrates that the atrial-fibrillation-associated gene GREM2 regulates cardiac laterality, cardiomyocyte differentiation and atrial rhythm. Disease Models \& Mechanisms, 6 (2), 332-341.

(35) Marvin, M.J., Di Rocco, G., Gardiner, A. et al. (2001) Inhibition of Wnt activity induces heart formation from posterior mesoderm. Genes and Development, 15 (3), 316-327.

(36) Lavery, D.L., Martin, J., Turnbull, Y.D., and Hoppler, S. (2008) Wnt6 signaling regulates heart muscle development during organogenesis. Developmental Biology, 323 (2), 177-188.

(37) Gibb, N., Lavery, D.L., and Hoppler, S. (2013) sfrp1 promotes cardiomyocyte differentiation in Xenopus via negative-feedback regulation of Wnt signalling. Development, 140 (7), 1537-1549.

(38) Ueno, S., Weidinger, G., Osugi, T. et al. (2007) Biphasic role for Wnt/beta-catenin signaling in cardiac specification in zebrafish and embryonic stem cells. Proceedings of the National Academy of Sciences of the United States of America, 104 (23), 9685-9690. 
(39) Naito, A.T., Shiojima, I., Akazawa, H. et al. (2006) Developmental stage-specific biphasic roles of Wnt/beta-catenin signaling in cardiomyogenesis and hematopoiesis. Proceedings of the National Academy of Sciences of the United States of America, 103 (52), 19812-19817.

(40) Ni, T.T., Rellinger, E.J., Mukherjee, A. et al. (2011) Discovering small molecules that promote cardiomyocyte generation by modulating Wnt signaling. Chemistry and Biology, 18 (12), 1658 1668.

(41) Wang, H., Hao, J., and Hong, C.C. (2011) Cardiac induction of embryonic stem cells by a small molecule inhibitor of Wnt/ $\beta$-catenin signaling. ACS Chemical Biology, 6 (2), 192-197.

(42) Minami, I., Yamada, K., Otsuji, T.G. et al. (2012) A small molecule that promotes cardiac differentiation of human pluripotent stem cells under defined, cytokine- and xeno-free conditions. Cell Reports, 2 (5), 1448-1460.

(43) Willems, E., Spiering, S., Davidovics, H. et al. (2011) Small-molecule inhibitors of the Wnt pathway potently promote cardiomyocytes from human embryonic stem cell-derived mesoderm. Circulation Research, 109 (4), 360-364.

(44) Ren, Y., Lee, M.Y., Schliffke, S. et al. (2011) Small molecule Wnt inhibitors enhance the efficiency of BMP-4-directed cardiac differentiation of human pluripotent stem cells. Journal of Molecular and Cellular Cardiology, 51 (3), 280-287.

(45) Saraswati, S., Alfaro, M.P., Thorne, C.A. et al. (2010) Pyrvinium, a potent small molecule Wnt inhibitor, promotes wound repair and post-MI cardiac remodeling. PLoS One, 5 (11), e15521.

(46) Uccelli, A., Moretta, L., and Pistoia, V. (2008) Mesenchymal stem cells in health and disease. Nature Reviews Immunology, 8 (9), 726-736.

(47) Bennett, C.N., Longo, K.A., Wright, W.S. et al. (2005) Regulation of osteoblastogenesis and bone mass by Wnt10b. Proceedings of the National Academy of Sciences of the United States of America, 102 (9), 3324-3329.

(48) Bennett, C.N., Ouyang, H., Ma Y.L. et al. (2007) Wnt10b increases postnatal bone formation by enhancing osteoblast differentiation. The Journal of Bone and Mineral Research of the American Society for Bone and Mineral Research, 22 (12), 1924-1932.

(49) Kang, S., Bennett, C.N., Gerin, I. et al. (2007) Wnt signaling stimulates osteoblastogenesis of mesenchymal precursors by suppressing CCAAT/enhancer-binding protein alpha and peroxisome proliferator-activated receptor gamma. Journal of Biological Chemistry, 282 (19), $14515-14524$.

(50) Cawthorn, W.P., Bree, A.J., Yao, Y. et al. (2012) Wnt6, Wnt10a and Wnt10b inhibit adipogenesis and stimulate osteoblastogenesis through a $\beta$-catenin-dependent mechanism. Bone, 50 (2), 477 489.

(51) Longo, K.A., Wright, W.S., Kang, S. et al. (2004) Wnt10b inhibits development of white and brown adipose tissues. Journal of Biological Chemistry, 279 (34), 35503-35509.

(52) D'Alimonte, I., Lannutti, A., Pipino, C. et al. (2013) Wnt signaling behaves as a "master regulator" in the osteogenic and adipogenic commitment of human amniotic fluid mesenchymal stem cells. Stem Cell Reviews, 9, 642-654.

(53) Christodoulides, C., Scarda, A., Granzotto, M. et al. (2006) WNT10B mutations in human obesity. Diabetologia, 49 (4), 678-684.

(54) Kim, I.C., Cha, M.H., Kim, D.M. et al. (2011) A functional promoter polymorphism $-607 \mathrm{G}>\mathrm{C}$ of WNT10B is associated with abdominal fat in Korean female subjects. The Journal of Nutritional Biochemistry, 22 (3), 252-258.

(55) Van Camp, J.K., Beckers, S., Zegers, D. et al. (2012) Genetic association between WNT10B polymorphisms and obesity in a Belgian case-control population is restricted to males. Molecular Genetics and Metabolism, 105 (3), 489-493.

(56) Katoh, M. and Katoh, M. (2007) AP1- and NF-kappaB-binding sites conserved among mammalian WNT10B orthologs elucidate the TNFalpha-WNT10B signaling loop implicated in carcinogenesis and adipogenesis. International Journal of Molecular Medicine, 19 (4), 699 703.

(57) Saitoh, T., Kirikoshi, H., Mine, T., and Katoh, M. (2001) Proto-oncogene WNT10B is upregulated by tumor necrosis factor alpha in human gastric cancer cell line MKN45. International Journal of Oncology, 19 (6), 1187-1192. 
(58) Chang, J., Liu, F., Lee, M. et al. (2013) NF-кB inhibits osteogenic differentiation of mesenchymal stem cells by promoting $\beta$-catenin degradation. Proceedings of the National Academy of Sciences of the United States of America, 110 (23), 9469-9474.

(59) Chang, J., Sonoyama, W., Wang, Z. et al. (2007) Noncanonical Wnt-4 signaling enhances bone regeneration of mesenchymal stem cells in craniofacial defects through activation of p38 MAPK. Journal of Biological Chemistry, 282 (42), 30938-30948.

(60) Liu, N., Shi, S., Deng, M. et al. (2011) High levels of $\beta$-catenin signaling reduce osteogenic differentiation of stem cells in inflammatory microenvironments through inhibition of the noncanonical Wnt pathway. The Journal of Bone and Mineral Research of the American Society for Bone and Mineral Research, 26 (9), 2082-2095.

(61) Alfaro, M.P., Pagni, M., Vincent, A. et al. (2008) The Wnt modulator sFRP2 enhances mesenchymal stem cell engraftment, granulation tissue formation and myocardial repair. Proceedings of the National Academy of Sciences of the United States of America, 105 (47), 18366-18371.

(62) Alfaro, M.P., Vincent, A., Saraswati, S. et al. (2010) sFRP2 suppression of bone morphogenic protein (BMP) and Wnt signaling mediates mesenchymal stem cell (MSC) self-renewal promoting engraftment and myocardial repair. Journal of Biological Chemistry, 285 (46), 3564535653.

(63) Mirotsou, M., Zhang, Z., Deb, A. et al. (2007) Secreted frizzled related protein 2 (Sfrp2) is the key Akt-mesenchymal stem cell-released paracrine factor mediating myocardial survival and repair. Proceedings of the National Academy of Sciences of the United States of America, 104 (5), 1643-1648.

(64) Mirotsou, M., Jayawardena, T.M., Schmeckpeper, J. et al. (2011) Paracrine mechanisms of stem cell reparative and regenerative actions in the heart. Journal of Molecular and Cellular Cardiology, 50 (2), 280-289.

(65) Clements, W.K., Kim, A.D., Ong, K.G. et al. (2011) A somitic Wnt16/Notch pathway specifies haematopoietic stem cells. Nature, 474 (7350), 220-224.

(66) Luis, T.C. and Staal, F.J.T. (2009) WNT proteins: environmental factors regulating HSC fate in the niche. Annals of the New York Academy of Sciences, 1176, 70-76.

(67) Staal, F.J.T. and Sen, J.M. (2008) The canonical Wnt signaling pathway plays an important role in lymphopoiesis and hematopoiesis. European Journal of Immunology, 38 (7), 1788-1794.

(68) Reya, T., Duncan, A.W., Ailles, L. et al. (2003) A role for Wnt signalling in self-renewal of haematopoietic stem cells. Nature, 423 (6938), 409-414.

(69) Scheller, M., Huelsken, J., Rosenbauer, F. et al. (2006) Hematopoietic stem cell and multilineage defects generated by constitutive beta-catenin activation. Nature Immunology, 7 (10), 1037 1047.

(70) Kirstetter, P., Anderson, K., Porse, B.T. et al. (2006) Activation of the canonical Wnt pathway leads to loss of hematopoietic stem cell repopulation and multilineage differentiation block. Nature Immunology, 7 (10), 1048-1056.

(71) Zhao, C., Blum, J., Chen, A. et al. (2007) Loss of beta-catenin impairs the renewal of normal and CML stem cells in vivo. Cancer Cells, 12 (6), 528-541.

(72) Malhotra, S., Baba, Y., Garrett, K.P. et al. (2008) Contrasting responses of lymphoid progenitors to canonical and noncanonical Wnt signals. Journal of Immunology (Baltimore, MD: 1950), 181 (6), 3955-3964.

(73) Nemeth, M.J., Topol, L., Anderson, S.M. et al. (2007) Wnt5a inhibits canonical Wnt signaling in hematopoietic stem cells and enhances repopulation. Proceedings of the National Academy of Sciences of the United States of America, 104 (39), 15436-15441.

(74) Louis, I., Heinonen, K.M., Chagraoui, J. et al. (2008) The signaling protein Wnt4 enhances thymopoiesis and expands multipotent hematopoietic progenitors through beta-cateninindependent signaling. Immunity, 29 (1), 57-67.

(75) Luis, T.C., Naber, B.A.E., Roozen, P.P.C. et al. (2011) Canonical wnt signaling regulates hematopoiesis in a dosage-dependent fashion. Cell Stem Cell, 9 (4), 345-356.

(76) Luis, T.C., Ichii, M., Brugman, M.H. et al. (2012) Wnt signaling strength regulates normal hematopoiesis and its deregulation is involved in leukemia development. Leukemia, 26 (3), 414-421. 
(77) Hochedlinger, K., Yamada, Y., Beard, C., and Jaenisch, R. (2005) Ectopic expression of Oct-4 blocks progenitor-cell differentiation and causes dysplasia in epithelial tissues. Cell, 121 (3), 465-477.

(78) Lee, S.M., Tole, S., Grove, E., and McMahon, A.P. (2000) A local Wnt-3a signal is required for development of the mammalian hippocampus. Development (Cambridge England), 127 (3), 457-467.

(79) Lie, D.-C., Colamarino, S.A., Song H.-J. et al. (2005) Wnt signalling regulates adult hippocampal neurogenesis. Nature, 437 (7063), 1370-1375.

(80) Chenn, A. and Walsh, C.A. (2002) Regulation of cerebral cortical size by control of cell cycle exit in neural precursors. Science, 297 (5580), 365-369.

(81) Bautch, V.L. (2011) Stem cells and the vasculature. Nature Medicine, 17 (11), 1437-1443.

(82) Medici, D. and Kalluri, R. (2012) Endothelial-mesenchymal transition and its contribution to the emergence of stem cell phenotype. Seminars in Cancer Biology, 22 (5-6), 379-384.

(83) Medici, D., Shore, E.M., Lounev, V.Y. et al. (2010) Conversion of vascular endothelial cells into multipotent stem-like cells. Nature Medicine, 16 (12), 1400-1406.

(84) Zeisberg, E.M., Tarnavski, O., Zeisberg, M. et al. (2007) Endothelial-to-mesenchymal transition contributes to cardiac fibrosis. Nature Medicine, 13 (8), 952-961.

(85) Zeisberg, E.M., Potenta, S., Xie, L. et al. (2007) Discovery of endothelial to mesenchymal transition as a source for carcinoma-associated fibroblasts. Cancer Research, 67 (21), 10123 10128.

(86) Medici, D., Potenta, S., and Kalluri, R. (2011) Transforming growth factor- $\beta 2$ promotes Snailmediated endothelial-mesenchymal transition through convergence of Smad-dependent and Smad-independent signalling. Biochemical Journal, 437 (3), 515-520.

(87) Markwald, R.R., Fitzharris, T.P., and Smith, W.N.A. (1975) Structural analysis of endocardial cytodifferentiation. Developmental Biology, 42 (1), 160-180.

(88) Aisagbonhi, O., Rai, M., Ryzhov, S. et al. (2011) Experimental myocardial infarction triggers canonical Wnt signaling and endothelial-to-mesenchymal transition. Disease Models \& Mechanisms, 4 (4), 469-483.

(89) Armulik, A., Abramsson, A., and Betsholtz, C. (2005) Endothelial/pericyte interactions. Circulation Research, 97 (6), 512-523.

(90) Moonen, J.-R.A.J., Krenning, G., Brinker, M.G.L. et al. (2010) Endothelial progenitor cells give rise to pro-angiogenic smooth muscle-like progeny. Cardiovascular Research, 86 (3), 506-515.

(91) Cheng, S.-L., Shao, J.-S., Behrmann, A. et al. (2013) Dkk1 and Msx2-Wnt7b signaling reciprocally regulate the endothelial-mesenchymal transition in aortic endothelial cells. Arteriosclerosis, Thrombosis, and Vascular Biology, 33 (7), 1679-1689.

(92) Hirata-Tominaga, K., Nakamura, T., Okumura, N. et al. (2013) Corneal endothelial cell fate is maintained by LGR5 through the regulation of hedgehog and Wnt pathway. Stem Cells, 31 (7), 1396-1407.

(93) Das Gupta, R. and Fuchs, E. (1999) Multiple roles for activated LEF/TCF transcription complexes during hair follicle development and differentiation. Development (Cambridge England), 126 (20), 4557-4568.

(94) Gregorieff, A. and Clevers, H. (2005) Wnt signaling in the intestinal epithelium: from endoderm to cancer. Genes and Development, 19 (8), 877-890. 


\title{
2 \\ Directed Cardiomyogenesis of Pluripotent Stem Cells
}

\author{
Jeffery B. Bylund ${ }^{1}$ and Antonis K. Hatzopoulos ${ }^{2}$ \\ ${ }^{I}$ Department of Medicine and Department of Pharmacology, Vanderbilt University, USA \\ ${ }^{2}$ Department of Medicine, Vanderbilt University, USA
}

\subsection{Introduction}

Generating cardiac tissue ex vivo to replace damaged or failing myocardium has been a goal of researchers for decades. Recent advances in stem-cell biology allowing the isolation and culture of pluripotent stem cells have made this aspiration a possibility. The complexity of the processes that drive stem cells from a pluripotent state toward progenitor and then specialized cell populations has made in vitro derivation of cardiomyocytes a challenging pursuit. However, a better understanding of the processes involved in cardiac tissue formation on a cellular and molecular level has unlocked the mechanisms that transform a pool of cells in the mid-portion of the embryo into a beating tube and finally a highly efficient, four-chambered double pump. Advances in our understanding of and ability to culture pluripotent stem cells have provided a model system to which to apply our more recent understanding of cardiac developmental mechanisms in vitro. This application has further refined our understanding of the molecular mechanisms behind the cardiomyogenic process and allowed for the creation of large numbers of functional cardiomyocytes in culture. These cultured cardiomyocytes have applications in the fields of drug testing and toxicology, basic research, and eventually as novel therapeutics.

In order to illustrate the complexity and exquisite delicacy of the processes whereby heart tissue is formed, we will begin this chapter in vivo, learning from the embryo how a heart

Chemical Biology in Regenerative Medicine: Bridging Stem Cells and Future Therapies, First Edition. Edited by Charles C. Hong, Ada S. Ao, and Jijun Hao.

(C) 2014 John Wiley \& Sons, Ltd. Published 2014 by John Wiley \& Sons, Ltd. 
is made. This will include a look at the major morphological features of the developing heart and at the timing of their appearance. We will then provide a summary of the major cellular and molecular events involved in the formation of each important feature.

After establishing a biological context for the varied events that take place during cardiogenesis, we will move out of the embryo and into the cell culture dish. In this section we will review the different types of pluripotent stem cell and their basic biology, including how they differ from other types of stem cell and what features make them important for the modeling of cardiac development in vitro.

The third section will cover the approaches used to generate cardiomyocytes from pluripotent stem cells. This will include strategies for using biological molecules such as growth factors to modulate signaling pathways in an attempt to mimic key developmental signaling events. This section will also cover the use of small molecules and other approaches to modulating these same key developmental pathways.

The final section will discuss the applications for stem cell-derived cardiomyocytes. This will include a look at the use of pluripotent stem cell-derived cardiomyocytes in basic research, drug discovery, tissue engineering, and clinical applications.

\subsection{A Brief Review of Heart Development}

\subsubsection{Cellular and Morphological Movements}

While our understanding of the anatomical and morphological features of heart development spans more than a century, it is only recently that we have begun to unlock the cellular and molecular mechanisms of this amazingly complex process. In this section we will review how a small number of progenitor cells in the early embryo are able to transform into a four-chambered, life-giving pump.

Before any organs begin to form, the developing embryo must transition from a mass of cells into layers of tissue that will eventually form a complete organism. The process by which these primitive layers of tissue form is known as gastrulation. At gastrulation the embryo transforms from a sphere of dividing cells into an organized, multilayered disk (human) or tube (mouse) called a gastrula [1,2]. The position of each layer of tissue generally lends itself to the eventual positioning of the various organs. The skin will form from the topmost outer layer, the ectoderm, while the inner and bottom layers, the endoderm and mesoderm, will form the gut, the muscles, the heart, and other organs. It is after gastrulation that the heart begins to form from the mesodermal layer.

In the post-gastrulation embryo the heart is the first organ to form and is the first functional organ, pumping blood and providing nutrients to diffusion-limited cells as the embryo develops. The complete heart is made up of several cell and tissue types. Innermost is the endocardium, a layer of endothelial cells that line the chambers and major vessels coming into and stemming from the base of the heart. In the middle of the wall of the heart is the largest portion by volume and mass, the myocardium. The myocardium consists of cardiomyocytes, which are responsible for the contractile force of the heart and for the propagation of membrane depolarization that drives regular beating. The myocardium is composed of a combination of several types of cardiomyocyte, each of which is functionally and structurally distinct. These distinctions are the result of the functionally distinct role of each region of the myocardium during contraction. The atria are primarily responsible for 
regulating blood flow into and out of the ventricles, helping to maintain a proper rhythm. The ventricles provide most of the contractile force generated during a heartbeat. Connecting the atria and ventricles are bundles of mostly "nonworking" cardiomyocytes that initiate and propagate the electrical signals which cause the heart to beat. These nonworking cells are generally referred to as conduction system cells. The outermost layer of the heart is the epicardium. This is a layer of connective tissue that serves as a covering and provides mainly smooth muscle cells to support the coronary arteries. During development, each of these layers must form at the right time and in the right place in order to provide a fully formed and functionally normal heart.

While the heart is not fully grown until after birth, achieving a mostly complete heart takes a remarkably short amount of time in lower mammals. In the well-studied mouse embryo the progression from progenitor cells to a functional four-chambered heart takes only about a week [3]. Arriving at this final configuration requires passage through what may be described as four major stages: specification of early cardiac progenitors and their coalescence into cardiogenic regions called heart fields; fusion of these cells at the midline to form the heart tube; cardiac looping and primitive chamber formation; and finally cardiac remodeling, including septation and valve formation.

The first of these four stages of heart development is the specification and migration of cardiac progenitor cells from the mesoderm of the gastrula. These early cardiogenic regions appear around embryonic day 6.5 in mice, at Hamburger Hamilton stage 3 in chicks and about 22 days postfertilization in humans [4,5]. At the earliest stages, cardiac progenitors are not easily distinguished from other cells in the mesoderm. However, by day 7.5 in the mouse these progenitors have migrated into the midline of the embryo to form the first distinguishable cardiac structure, the cardiac crescent $[3,6]$. This crescent, shaped like a $\mathrm{C}$ with the open end oriented caudally, is located at the cranial portion of the embryo just below the developing head fold and neural tube. At the cardiac crescent stage cardiac progenitor cells reside in two distinct regions, called the primary and secondary heart fields (PHF and SHF, respectively) [6]. The cells in these fields will eventually become most of the heart. Cells in the PHF eventually become the left ventricle and a portion of the atria and the right ventricle. The SHF is located lateral to the PHF and contributes to the rest of the atria and right ventricle, as well as the entire outflow tract. In addition to their spatial regulation, the cells of the PHF and SHF are also temporally regulated. The cells derived from the PHF are the first to differentiate into cardiomyocytes and are known as first lineage cells. The SHF cells remain in an undifferentiated state until after the PHF cells begin to form the heart tube. During tube formation and throughout looping, SHF cells continue to differentiate and make contributions to the atria, ventricles, and outflow tract [7].

The second stage of heart development is cardiac tube formation. This process starts around embryonic day 8 in mice and is indicated morphologically as a budlike structure emerges at the cranial portion in the PHF of the cardiac crescent [3]. This budlike structure then enlarges as the crescent begins to fuse at the midline, gaining volume while expanding cranially and caudally [8]. At the caudal end of the crescent PHF, cells migrate downward in a bisymmetric fashion on the right and left sides of the emerging heart, forming an inverted Y. At the top of this inverted Y the budding PHF cells, with contributions from the SHF, expand downward to form the heart tube. This tube is a cylindrical structure composed of developing myocardium on the outside and a layer of endothelium called endocardium on the interior. While the PHF is expanding to form the anterior portions of the emerging 
heart, the SHF is also expanding cranially and posterior to the PHF. The expanding SHF cells make some contribution to the mostly PHF-derived heart tube cells but also form a distinct structure at the top of the inverted $Y$ that will eventually become the outflow tract. The cranial and caudal portions of this tube are known respectively as the arterial and venous poles. Once the heart tube is completely fused between the correctly oriented arterial and venous poles, it begins to act as a rudimentary pump. This pumping action is essential for moving blood through the developing circulatory system of the embryo, which at this point requires blood flow to provide nutrients and oxygen to diffusion-limited cellular compartments. It is also evident that myocardial cells capable of producing contractions are present even in this rudimentary heart.

Though the heart is beginning to fulfill its functional role as a pump to move blood through the embryo, it must still detach itself from the mediastinum and orient itself properly for chamber and valve formation. This reorientation process occurs during the third major stage in heart development, cardiac looping. In mice this looping process begins around embryonic day 9.0 and in humans around 5 weeks postfertilization. In mice it takes about 1-2 days to complete. As already described, the newly formed heart tube is at this point an asymmetric structure consisting of a bifurcated region at the venous pole and a straight tube with rudimentary SHF-derived outflow tract at the arterial pole. The bifurcated caudal end contains the inflow tract and what will become the atria. During looping, this pole of the heart moves dorsally and cranially while twisting to the right, bringing it to a cranial position just above the developing ventricles. While the venous pole is reorienting as described, the arterial pole containing the outflow tract is also moving, albeit caudally and ventrally in an opposite and complementary fashion. These movements have now oriented the two poles in an asymmetric dorsal-ventral configuration with the outflow tract on the right and a common atrium and inflow tract on the left. These two regions are connected by a loop of bulbous, asymmetric cardiac tissue that includes the atrioventricular (AV) canal. This bulbous loop will eventually become the left and right ventricles. The former poles will become the atria and major vessels located at the base of the heart. It is during this period of development that the ventricles begin to be separated via the interventricular septum and that the precursors to the valves, or endocardial cushions, begin to form [3, 8].

Now that the heart is positioned properly, it is ready to proceed to the final step of development, cardiac remodeling and definitive formation of the cardiac chambers. As indicated earlier, primitive chambers are already aligned and ready to form the two atria and two ventricles characteristic of the adult heart. The atria form as the common atrium expands to form two appendages. While these atrial appendages are forming, the outflow tract is imposed upon their junction by continued spiraling of the heart tube. The outflow tract and atria fuse, producing two distinct atrial chambers. At the same time the inflow region insinuates itself between the bulging ventricular regions on the dorsal side of the heart. The inflow tract then fuses with the cardiac loop, creating separate left and right ventricles. During this stage the valves and septa that separate the chambers from one another are also forming. The endocardial cushions in the AV canal give rise to the tricuspid and mitral valves, while those in the outflow tract form the septum dividing the outflow tract into the aorta and pulmonary artery [3].

These four steps give a sketch of the complexity of the heart-forming process. Indeed, the procession through these four stages is complete by around E14 in the mouse, yet the heart continues to grow and mature through the remaining week or so until birth, and even 
thereafter. For the purpose of this chapter, however, these four basic steps provide the proper context for our discussion of the cell types that populate the heart during development and the molecular mechanisms that drive them. It is this molecular understanding, gained by careful inspection of the different stages of heart development, that has allowed us to generate cardiomyocytes in vitro from pluripotent stem cells.

\subsubsection{Molecular Events in Heart Development}

In order to appreciate how our understanding of the major events in cardiogenesis has been applied to pluripotent stem-cell differentiation in vitro, it is important to describe the major cell types involved in each of the developmental stages of the heart in terms of their origin, movement, and eventual fate. Coordinating the differentiation and movement of cells during the four stages are major developmental signaling pathways. From mesoderm derivation to remodeling and chamber formation, these signaling pathways act to sequentially regulate cardiac transcription-factor networks. These transcription-factor networks regulate important structural and functional genes that eventually characterize the mature cardial cells. Thus the molecular and cellular events that drive cardiogenesis are best thought of in terms of these signaling pathways and the transcription factors under their control. In the context of cardiomyocyte differentiation from pluripotent stem cells, signals and factors regulating the origin and differentiation of early cardiac progenitor cells are most relevant. We will therefore focus most of our attention on the early events of cardiac differentiation.

\subsubsection{Molecular Events of Mesoderm Derivation}

Prior to gastrulation, stem cells from the inner cell mass (ICM) of the blastula form one of the earliest embryonic tissue types, the epiblast. Cells of the epiblast then give rise to the three primary germ layers. They first form the primitive streak and the earliest of the three germ layers, the ectoderm. The cells of the primitive streak then go on to form the endoderm and mesoderm. This transition of embryonic stem cells toward mesodermal tissue may be classified as the first important transition in vivo that guides our understanding of how to generate cardiomyocytes in vitro from pluripotent stem cells.

As gastrulation progresses, epiblast cells migrate through the primitive streak and expand outward between the ectoderm and endoderm [9]. Mesodermal cells continue to expand laterally into the developing space between the ectoderm and endoderm, eventually segmenting into four different sections [10]. Developmental pathways directing the specification, migration, and expansion of mesoderm from epiblast through the primitive streak and into its final position between ectoderm and endoderm include Activin/Nodal, Wnt, BMP, and fibroblast growth factor (FGF) [9, 11]. These pathways act in concert to regulate each otherconfining proper activation to specific regions by regulating the expression of agonists and antagonists - and to regulate the expression of mesodermal transcription factors.

Early induction of mesoderm is associated with the activity of Activin/Nodal signaling. Nodal is expressed in the epiblast, where it activates BMP signaling, which in turn activates Wnt. Later, Nodal and BMP act together to control the expression of Wnt antagonists such as Dkk1 in order to restrict Wnt signaling to the primitive streak, where it directs the formation of mesoderm. During mesoderm induction the Wnt signaling pathway acts as a fulcrum point for the interaction of Nodal and BMP signaling. As mesodermal tissue continues to form, Wnt signaling is detected toward the posterior end of the primitive streak 
around E6.5 and then expands into the entire primitive streak and much of the mesoderm by E7.5 [12]. Loss of Wnt signaling components, including Wnt ligands, co-receptors, and $\beta$-catenin (the nuclear protein mediating Wnt transcriptional control), results in a failure to form mesodermal tissue [13-17].

Wnt signaling affects the formation of mesoderm mainly via the Wnt ligand Wnt3A. Wnt3A regulates the activation of the essential mesodermal transcription factors T-brachyury (T-bry) and Eomes [18]. In fact, Wnt signaling acts directly on T-bry via translocation of $\beta$-catenin to the nucleus and activation of gene transcription [19]. These transcription factors play a critical role in cell migration during mesoderm specification and are used as definitive markers of mesodermal tissue [20].

FGF signaling also plays an essential role in the early formation of mesoderm. Loss of FGF ligands such as FGF8 and receptors such as FGFR1 leads to a loss of mesoderm by interfering with cell migration [21-23]. The effect of FGF signaling on mesoderm formation is likely mediated via its regulation of the transcription factors Snail, T-bry, and Tbx6, three genes required for mesoderm migration, specification, and patterning.

This gives a brief sketch of the molecular events required for mesoderm formation, providing context for the next section, where we will examine the formation of cardiac progenitors via the transcription factors that drive cardiac progenitors and the signaling pathways that guide them. It is important to note that mesoderm formation and cardiacprogenitor specification are not necessarily mutually exclusive processes. Many important developmental events occur in concert with one another as the heart develops. Examples of this concept will be apparent as we continue our discussion of the cellular and molecular events involved in cardiac development.

\subsubsection{Transcription Factors in Cardiac Development}

Cardiac precursors can be detected in the anterior region of the primitive streak during the early stages of mesoderm formation. These precursors are not yet cardiac cells but are a collection of cardiac progenitors that give rise to the earliest cardiac cells. These early cardiac cells are present on either side of the embryonic midline and express the transcription factors Mesp-1 and Mesp-2. Loss of Mesp expression in these cells causes a failure in the migration required for the movement of emerging cardiac progenitor cells $[8,24,25]$.

Expression of Mesp-1 in the cardiogenic mesoderm is a master signal that initiates the cardiac transcription-factor signaling network [24]. Mesp-1 expression rapidly induces the expression of a new set of transcription factors involved in cardiogenesis. These include Nkx2-5, Hand2, Myocd, Gata4, Mef2c, Tbx20, FoxH1, Foxc1, SRF, and Foxc2, among others [24]. The mechanism behind this activation lies in the ability of Mesp-1 to bind directly within the regulatory regions of its target genes, including Nkx2-5, Hand2, and Myocd. Myocd in turn activates Mef2c and SRF, which go on to activate the essential cardiac structural genes Myh6, Myl1, Myl2, Myl7, and cardiac troponin 2 (Tnnt2) [26,27].

Each of the transcriptional events in the formation of cardiac progenitors is thereby linked from Mesp-1 expression in the early mesoderm on through expression of cardiac-specific structural and functional components in mature cardiomyocytes. This point is very important to keep in mind: cardiogenesis on a cellular level occurs via the carefully orchestrated, stepwise movement of progenitor cells toward mature cardiac subtypes, directed by cardiac 
transcription factors. As we will discuss later, it is upon this principle that most protocols for production of de novo cardiomyocytes from pluripotent stem cells depend.

This summary illustrates the sequence of transcription-factor events kicked off by Mesp1 and propagated onward to drive heart formation. We will now discuss where these transcription factors are expressed and the role they play during cardiogenesis. Some are restricted temporally and spatially to a particular period of heart development, while others are expressed throughout.

Nkx2-5, Hand1 and 2, Gata4, and Tbx1,5 and 20 are expressed in the PHF. These transcription factors direct the differentiation of cells within the PHF as they populate the heart tube and differentiate during looping morphogenesis $[3,6,8] . \mathrm{Nkx} 2-5$ is important in atrial-ventricular chamber compartmentalization and the formation of ventricular tissue. Hand 1 and 2 play a direct role in the formation of the left and right ventricles, respectively. Gata4 expression is essential for looping morphogenesis, septation of the valves, and formation of ventricular myocardium. At the stage of heart-tube formation, cardiac cells must migrate and fuse at the midline. Here, Gata4 and a companion transcription factor, Foxp4, are essential for proper tube formation, as mutations in these genes lead to a failure of the cells to fuse and form a proper heart tube [3].

The Tbx transcription factors are an important class that play diverse roles in cardiogenesis, including patterning and specification of conduction system cells and sinoatrial nodal cells and distinguishing between atrial and ventricular myocardium. In fact, patterning via the Tbx genes is the major driver for distinguishing between working and nonworking myocardium during the later stages of cardiac development [28]. Tbx transcription factors are also critical to the formation of the outflow and inflow tracts and neuralization of the atrium at later stages of heart development $[29,30]$. Tbx20 plays an important role in proliferation of myocardial progenitor cells during early myocardial expansion. Mutant embryos lacking Tbx20 expression present a large decrease in the number of terminally differentiated cardiomyocytes [31]. Tbx 18 is expressed in the extreme posterior regions of the SHF [6]. Cells that express Tbx18 end up in diverse cardiac locations, from smooth muscle to myocardium and interventricular septum [6].

Isl1 is another essential cardiac transcription factor. Isl1 expression in the SHF plays a role in many cardiac development processes. Mutations that inactivate or knockdown its expression result in major cardiac defects. These include lack of septation in the atrial and ventricular compartments, abnormal atria, and a lack of outflow tract [32]. Is11, along with Gata4, can directly control the expression of Mef2c by binding a genomic enhancer element upstream of its coding sequence [33]. Incidentally, Isl1 expression has been detected in the PHF as well as the SHF (where it was originally identified), although it does not seem to be required for formation of the PHF lineages [34]. When Isl1 expression is knocked down in transgenic mice, the PHF and its associated derivatives still form as expected [32].

The Forkhead transcription factors (known as Fox) are also important for cardiac development. Foxh1 is expressed in the anterior region of the SHF and plays a role in formation of the outflow tract and right ventricle [35]. Foxh transcription factors also play a role in regulating other transcription factors, such as Mef2c [36]. Mef2c is involved in the formation of the outflow tract, inflow tract and proper development of the right ventricle [37].

Pitx transcription factors are also required for myocardial formation. Pitx $2 c$ expression is detectable throughout the left side of the SHF during early cardiogenesis and is later detected extensively in the ventricular and atrial myocardium. Pitx $2 \mathrm{c}$ is essential in the formation of 
left-right asymmetry in the heart and loss of Pitx2 expression leads to right atrial isomerism and defects in ventricular maturation [38]. Pitx2c also regulates the formation of left versus right atrial chambers by repression of right atrial identity.

Atrial and ventricular cells are being patterned at very early stages of cardiac development. Cells in the SHF that will eventually become part of the atria require the expression of the transcription factor NFAT in order to fully develop [39]. At later stages of heart development the transcription factors Irx 4 and Hey 2 are expressed in emerging ventricles and play a role in ventricular myocyte formation. The transcription factors Hey 1 and Coup-TFII are expressed in the atria, where they regulate atrial myocyte formation [28, 40, 41].

Transcription factors are a crucial component of cardiac development. Not only do they regulate the proliferation, patterning, differentiation, and final maturation of cardiomyocytes and their progenitors during development, they are also indispensable as markers of distinct differentiation stages from progenitor populations and mature cardiac subtypes. Understanding the role of transcription factors in tissue-specific compartments allows us to track the development of cardiomyocytes in vitro, thereby allowing the successful translation of developmental paradigms into the culture dish for efficient cardiomyocyte differentiation.

It is interesting to note that many regions of the heart are sensitive to perturbations of several transcription factors. In other words, no single transcription factor affects only one part of the complete heart. For example Nkx2-5, Hand1/2, and Tbx20 are all required for proper formation of the ventricles, yet their effects on ventricular tissue are not redundant. Loss of each of these transcription factors affects ventricular development in different ways [8]. This amazing interconnectedness of the transcriptional networks involved in cardiac development underscores their complexity.

\subsubsection{Major Developmental Signaling Pathways in Cardiac Development}

In order to coordinate the timing and expression of these networks, cells rely on the major developmental signaling pathways. Many of the same pathways already discussed as important for mesoderm formation are critical for the proper differentiation and maturation of cardiac progenitors. These pathways control diverse processes, including the expansion of progenitor populations and differentiation of progenitors into myocardial cells. In this section we will briefly discuss how the major developmental signaling pathways, Wnt, bone morphogenetic protein (BMP), Activin/Nodal, FGF, Retinoic acid (RA), and Shh, regulate cardiomyocyte development.

Wnt signaling is involved in almost all stages of heart development, from myocardial specification and early cardiac morphogenesis through heart-tube formation, looping, and chamber formation. Wnt ligands are expressed in the heart-forming fields during early heart development. As the heart fields merge to form the heart tube, different ligands are restricted to distinct regions of the heart, reflecting the diversity of function within even the same signaling family during differentiation [42]. Wnt receptors are also expressed in the heart fields. The expression of Wnt receptors in these tissues is just as diverse as the expression of Wnt ligands [42]. Functionally, Wnt exhibits pleiotropic effects on heart development. Canonical Wnt signaling is required for Isl1 induction and proliferation of cardiac progenitor cells but exerts an inhibitory effect on cardiac differentiation, while noncanonical JNK-mediated Wnt signaling promotes cardiac differentiation. Noncanonical 
Wnt signaling also regulates cell adhesion and cell polarity during cardiac morphogenesis [43].

As is the case with Wnt, BMP ligands and receptors are expressed in cardiac progenitor cells and the mature heart. BMP factors are secreted to cardiac progenitors by the endoderm during gastrulation as they move toward the cardiac crescent and SHF [44]. BMP2 induces the expression of Nkx2-5 in the cardiac crescent, which then directly interacts with the early BMP effector Smad to autoregulate BMP2 expression in the SHF [45]. BMP expression is also important in regulating elongation of the linear heart tube, heart-chamber formation, and cushion formation, as well as in interacting with Nodal signaling to establish leftright asymmetry [46]. These interactions between BMP and Nkx2-5 regulate early cardiac expansion by first stimulating proliferation and then limiting the number of cardiomyocytes in the heart tube to prevent overpopulation [34].

Nodal and Shh signaling regulate the activation of Pitx2c to control patterning of the left-right axis [6]. Without proper Shh signaling, the heart remains attached to the dorsal mesocardium, preventing proper detachment and looping [47]. Shh signals are expressed in cardiac progenitors that migrate through the SHF to form the subpulmonary myocardium and participate in atrial septation [48]. Shh restricts expression of Pitxc2 to the right side of the heart. Shh also interacts with Isl1, helping to regulate proliferation of progenitor cells and initiate differentiation [28].

FGF is another critical signaling pathway in various stages of cardiogenesis. FGF ligands and receptors are expressed in early cardiac progenitor cells and mature cardiac tissues [28]. FGF is also expressed in adjacent tissues and plays an inductive role during cardiomyocyte development. FGF interacts with BMP signaling to regulate the activity of Tbx transcription factors, thereby influencing the formation of both early cardiac progenitors and myocardial subtypes during differentiation. FGF and BMP also interact to regulate the differentiation of cardiac mesodermal cells between the myocardial and epicardial lineages [49, 50]. FGF is also required for proliferation of the SHF, formation of the arterial pole, and outflow-tract development [6].

RA signaling is a critical component of anterior-posterior patterning, the formation of the cardiac tube, and atrial-ventricular specification [51]. Expression of the RA receptor Raldh2 is required for heart-tube growth and outflow-tract formation. RA signaling is also important for stimulation of Mef2c expression via Gata4 [6].

The complexity of heart formation is reflected in the varied morphogenetic and cellular movements that take place during it, as well as in the transcription factors and developmental signaling pathways that drive them. While further research into the mechanisms of myocardial subtype specification and cardiac maturation and maintenance remains to be done, our current understanding of these processes allows their application toward the successful generation of cardiomyocytes in vitro from pluripotent stem cells.

\subsection{Introduction to Pluripotent Stem Cells}

\subsubsection{Unique Features of Pluripotent Stem Cells}

Stem cells in general possess two unique and defining properties that distinguish them from other cell types. The first is that they are self-renewing, able to proliferate practically 
indefinitely in their undifferentiated state to generate new pluripotent cells. The second defining property of stem cells is their ability to differentiate and form more mature, specialized cell types. In the case of adult stem cells, the capacity to differentiate into other cells types is confined to the subtypes required by the compartment in which they act (see Chapter 5 for more information). In contrast, pluripotent stem cells have a broader differentiation potential and can perhaps give rise to most tissue-specific cell types. These features make pluripotent stem cells an exciting source for the production of cardiomyocytes in vitro.

\subsubsection{Pluripotent Stem Cell Sources}

Pluripotent stem cells generally come from two major sources. The first are the embryonic stem cells (ESCs), which are derived from the inner-cell mass of blastocysts prior to implantation. These cells are both self-renewing and pluripotent, able to produce all the cell types of the body. Due to ethical issues and technical constraints, most early research conducted on ESCs in culture was focused on those isolated from mouse embryos. As technology has advanced and our ability to deal with the political and ethical concerns surrounding research using human embryos has evolved, such research has become less restricted, but it still poses many practical and ethical challenges. Research in mouse and human ESCs has identified a large network of transcription factors that are required for pluripotency, self-renewal, and suppression of differentiation [52,53]. In a seminal discovery by Yamanaka, it was shown that a forced expression of four of these factors Oct4, Klf4, Sox2, and c-Myc - is sufficient to reprogram somatic, terminally differentiated cells into pluripotent, embryonic-like stem cells. These reprogrammed cells were first derived from mouse embryonic and adult fibroblasts in 2006 using a subset of defined factors exogenously expressed in cultured cells [54]. A year later the same technique was shown to be effective at generating pluripotent stem cells from human adult fibroblasts $[55,56]$.

The second major source of pluripotent stem cells is the induced pluripotent stem cells (iPSCs), which have proven to be highly similar to ESCs in their ability both to self-renew and to differentiate into a variety of mature cell types [57]. iPSC technology offers the advantages of being both less ethically challenging and more patient-specific than the use of ESCs. Patient-specific stem cells open the way for generating cardiac tissue from patients suffering from genetic cardiac diseases in order to study these diseases in vitro, and eventually for the creation of patient-matched tissue that is resistant to immune rejection.

Since the initial reprogramming protocol, various modifications have been made to simplify and increase the efficiency of pluripotent stem-cell induction. These methods include using small molecules and proteins to aid the reprogramming process, generating new gene expression vectors that do not require viral integration, and modulating the regulation of pluripotency [58-64]. These advances have made generating iPSCs practical enough to be done in a routine fashion.

\subsubsection{Maintaining Pluripotency}

Prior to differentiation, stem cells are maintained in an undifferentiated, pluripotent state. Maintenance of this pluripotent state is critical for taking advantage of the self-renewing capacity of stem cells and for the efficient directed differentiation of cardiomyocytes in vitro 
[65]. If pluripotent stem cells begin to differentiate in culture prematurely, they rapidly lose their capacity for self-renewal and spontaneously acquire the features of differentiated cells in a more or less random fashion. In such a state they are ineffectual for use in directed differentiation. The tendency of pluripotent stem cells to spontaneously differentiate in culture must be kept in check until they are ready to be used for directed differentiation.

Both mouse and human cells have a tendency to spontaneously differentiate. Growth of pluripotent stem cells on feeder cells such as fibroblasts, also used for derivation of ESCs and iPSCs, helps to control this differentiation to some degree; however, use of feeders introduces an undefined component to the culture system, without even completely eliminating the differentiation.

The discovery that the protein LIF (leukemia inhibitory factor) was sufficient to prevent differentiation of mouse ESCs when added to the culture medium presented a major advancement to the field of ESC culture [66]. LIF is effective even in the absence of feeder cells and is therefore an essential component for the undifferentiated culture of mouse embryonic stem cells (mESCs) in feeder-free conditions. Culture of stem cells in the absence of feeders eliminates the variability associated with the undefined role they play in maintenance of pluripotency and their effect on the eventual fate of stem cells during differentiation. Use of LIF as an added factor in pluripotent stem-cell media also serves to illustrate the concept that the properties of pluripotent stem cells in culture can be directly controlled via the addition of defined factors to their culture medium.

It is critical to note that LIF is not sufficient to prevent spontaneous differentiation of cultured human pluripotent stem cells (hPSC). In order to support the feeder-independent culture of hPSCs more complex mixtures of defined factors have been developed. These formulations are based on our understanding of the signaling pathways that maintain pluripotency in vivo and include a combination of ligands such as bFGF and transforming growth factor beta-1 (TGF $\beta 1$ ) $[67,68]$. These strategies also employ artificial extracellular matrices such as matrigel or vitronectin in order to preserve pluripotency and enhance cell survival and attachment [69]. Feeder-free culture of human pluripotent cells in defined media allows for more precise control of pluripotency and differentiation [70].

In summary, the unlimited proliferative capacity and broad differentiation potential of pluripotent stem cells are defining characteristics that distinguish them from other stem cell types. These characteristics make them ideal for use as a source of cardiomyocytes in vitro. Both embryonic and adult tissues from mice and humans have been used as a source of pluripotent stem cells. Each of these stem cell types has its strengths and weaknesses in terms of both cell culture and differentiation potential for use in the production of cardiomyocytes, and it is critical that pluripotency is maintained prior to differentiation no matter the source or type.

\subsection{Cardiomyocyte Differentiation}

\subsubsection{Inducing Differentiation}

Upon induction of differentiation, cultured pluripotent stem cells proceed through a wellordered progression highly similar to that observed in the early stages of embryonic development in vivo [71]. This includes the sequential formation of cells with characteristics 
of mesodermal progenitors, mesoderm, cardiac mesoderm, cardiac progenitor cells, immature cardiomyocytes, and finally mature myocytes [70]. The differentiation of pluripotent stem cells into cardiomyocytes recapitulates the in vivo program with such fidelity it has been used to yield valuable insight into the basic mechanisms driving cardiac development $[12,72-74]$.

Tracking the expression of transcription factors characteristic of pluripotent cells, mesoderm, cardiac progenitors, and mature myocytes is an important example of how in vivo developmental paradigms aid in the derivation of cardiomyocytes from pluripotent stem cells. Over the first few days of differentiation, gene expression patterns indicate that pluripotency genes are downregulated while gastrulation gene expression is induced. Starting around day 2 and peaking at day 3 in mouse and a little later in human stem cells the mesodermal marker T-bry is expressed, indicating the formation of mesoderm $[65,75]$. After day 4 in both human and mouse stem cells, early cardiac markers such as Mesp-1 and Nkx2-5 can be detected. By day 10 more mature cardiac genes, including the light and heavy myosin chains, as well as cardiac conduction channels, are expressed. Expression of important genes for the developmental signaling pathways involved in cardiac development is also detected during this process. Thus pluripotent stem cells pass through the necessary developmental stages discussed in Section 2.1, which lead to cardiomyocyte formation [76].

In order to coax stem cells from their pluripotent state through each of these stages to yield cardiomyocytes, differentiation strategies typically focus on mimicking the in vivo differentiation process. The earliest of these techniques relies on the formation of embryoid bodies (EBs): small spheres of pluripotent cells induced to form in droplets of cell-suspension media hung on the lids of Petri dishes or forced to aggregate in microwells $[77,78]$. The strategy behind using such a system is to reconstruct the spherical nature of the early post-implantation embryo from which pluripotent stem cells are originally derived, in order to induce the process of gastrulation. In fact, differentiating EBs give rise to layers of tissue representative of mesoderm, endoderm, and ectoderm and will eventually produce cells representative of all the tissues of the body [79]. The EB method is used for the differentiation of both human and mouse pluripotent stem cells. Its effectiveness in both systems and its relative scalability have made it a popular format for induction of differentiation.

Monolayer differentiation techniques have also been used to induce the differentiation of pluripotent stem cells. These techniques, while not as scalable as EB or other suspension differentiation systems, have the advantage of being less complex, more accessible to proteins and other molecules used for cardiac induction, and more amenable for use in tissue engineering and other applications where thin films are preferable to aggregates [80,81]. Monolayer differentiation does not inherently produce embryonic germ-layer tissues as do EBs. These protocols thus rely heavily on the directed differentiation techniques discussed here in order to consistently generate significant numbers of cardiomyocytes.

\subsubsection{Directed Cardiomyogenesis}

EB formation alone is enough to induce some cardiogenesis. After induction of differentiation, small beating clusters are usually observed between days 8 and 10 of differentiation in $\mathrm{mESCs}$ and a little later in hPSCs $[80,82,83]$. However, only a small portion of cells 
in differentiating EBs will spontaneously become cardiomyocytes, usually around 5-10\% [84]. Noncardiomyocyte populations include other mesodermal lineages such as endothelial cells and smooth muscle cells and those of the other germ layers, including neurons. Thus merely forming EBs and allowing them to differentiate is not sufficient to generate large numbers of cardiomyocytes from pluripotent stem cells in vitro. As already mentioned, monolayers of pluripotent stem cells also form few if any cardiomyocytes when simply allowed to spontaneously differentiate in culture.

In order to induce more abundant populations of cardiomyocytes, further cues from embryonic development are used to drive or enhance the progression of cells to form mesoderm, cardiac progenitors, and functional cardiomyocytes. The most effective current methods employ a refined approach that relies on the addition of exogenous factors and small molecules in defined media at precise times during the course of differentiation [70]. These factors include protein and small-molecule agonists and antagonists of specific developmental signaling pathways, including Activin/Nodal, Wnt, BMP, FGF, and TGF $\beta$ $[69,70]$.

The first step in directing pluripotent stem cells toward becoming cardiomyocytes involves the induction of mesoderm. As discussed earlier in this chapter, one of the first events in the differentiation of cells into cardiomyocytes in vivo is the coordinated action of Activin/Nodal, BMP, and FGF signaling pathways to induce expression of the mesodermal transcription factors T-bry, Eomes, and SnaiI. Most successful protocols for the efficient induction of mesoderm therefore include addition of the Nodal ligand ActivinA, the BMP ligand BMP4, and the FGF ligand FGF2 [70]. Once mesoderm has been established, specification of cardiac progenitors can be induced using a combination of Wnt, TGF $\beta$, and BMP inhibitors with vascular-endothelial growth factor (VEGF). These molecules block the negative regulatory effect of their respective pathways at this stage of differentiation, allowing for the more robust transition of cardiac mesoderm to cardiac progenitor cells $[85,86]$. To drive cardiomyocyte differentiation from progenitor cells to functional, beating myocytes, VEGFA, FGF2, and the Wnt inhibitor Dkk1 are added. As when enhancing progenitor-cell specification, these factors maintain the positive regulatory activity of VEGF and FGF signaling while preventing the negative effects of Wnt on cardiomyocyte differentiation.

Other molecules outside of the traditional pathway modulators have also been empirically determined to enhance cardiomyocyte differentiation. These include vitamin $\mathrm{C}$, which enhances proliferation of cardiac progenitors, cardiogenic small molecules identified using high-throughput screening methods, and polymers such as PVA, which modify surface tension [87-89]. These molecules do not necessarily have developmental parallels but instead serve to illustrate the flexibility and utility of in vitro differentiation methods.

Despite the relatively high yield of cardiomyocytes produced using these protocols, there remains a significant amount of heterogeneity among the resultant cardiomyocytes. This heterogeneity presents a challenge for practical use of pluripotent stem cell-derived cardiomyocytes.

The first source of heterogeneity arises from the fact that even though cardiomyocyte populations of over $80 \%$ can be achieved by careful optimization of growth factor concentration and timing of treatment, the other $20 \%$ of cells are mainly mesoderm-derived cells such as endothelial cells [70]. Thus cardiomyocytes do not necessarily make up the entire population. To address this lack of homogeneity, purification strategies are employed to 
enrich the number of cardiomyocytes present in the culture by either selectively eliminating unwanted cell types by inserting antibiotic resistant cassettes behind cardiac genes or physically removing late cardiac progenitors using markers like KDR (VEGFR2) and NKX2-5 $[85,86,89]$. The advantage of using selection is that it yields the highest purity: up to $99 \%$ cardiomyocytes. Another source of heterogeneity among pluripotent stem cell-derived cardiomyocytes arises from the heterogeneous nature of cardiomyocytes themselves. Cells of the atria, ventricles, nodes, and conduction system have distinct functional and structural properties [82]. Pluripotent stem cell-derived cardiomyocyte populations contain both types of working myocardium as well as conduction-system and nodal myocardial cells [90]. In order to address this heterogeneity by directing differentiation toward specific cardiac subtypes, cues from development have guided early strategies for subtype specification. These protocols are in the early stages of research and remain to be fully exploited, but some early attempts have shown that this specification may be possible soon. Modification of differentiation protocols to modulate signaling pathways such as RA signaling can bias the differentiation protocol toward specific subpopulations of cardiomyocytes [91-93]. These protocols remain somewhat inefficient, however, and further research into the mechanisms that regulate cardiomyocyte subtype differentiation is required.

\subsection{Conclusion}

Applications for the use of hPSCs extend near and far. In the short term, use of iPSCs derived from patients with traditionally intractable cardiac illnesses will allow large numbers of disease- and patient-specific cardiomyocytes to be generated [94]. These myocytes can be used to study the pathology and development of these diseases in vitro, as well as to test drugs and other therapeutics for their efficacy. Research into the mechanisms of long QT syndrome and the effect of drugs on this phenotype, for example, has already yielded insights into the molecular mechanisms underlying this disease.

Another near-term application for hPSC-derived cardiomyocytes is in toxicology and drug screening. Most drugs fail in late stages of development and clinical trials, or are recalled after release due to unacceptable cardiac toxicity as an off-target effect [95]. By screening drugs in a human cardiomyocyte model system early in the drug development process, these off-target cardiotoxic effects can be discovered and development efforts can be shifted accordingly.

In the more long-term future, hPSC-derived cardiomyocytes hold promise as a source of cells for regenerative therapies. One can envision new hearts grown using cardiomyocytes derived from individual patient iPSCs, cardiac patches made from engineered myocytes on biopolymer scaffolds, and injection of engineered ventricular myocytes into post-ischemic tissues to replenish working myocardium.

Heart development is a complex process on the morphological, cellular, and molecular levels. Our understanding of these cellular and molecular mechanisms has improved greatly as molecular-biology and biochemical techniques have been applied to elucidate the once shadowy web of developmental signaling pathways and transcription-factor networks that drives cardiogenesis. This understanding has been applied to the directed cardiac differentiation of pluripotent stem cells in vitro. Continued research into the mechanisms behind cardiomyocyte maturation and subtype specification will combine with improved 
techniques to bring us closer to achieving the promising applications for pluripotent stem cell-derived cardiac myocytes.

\section{References}

(1) Beddington, R.S. and Robertson, E.J. (1999) Axis development and early asymmetry in mammals. Cell, 96 (2), 195-209.

(2) Hill, M.A. (2007) Early human development. Clinical Obstetrics and Gynecology, 50 (1), 2-9.

(3) Harvey, R.P. (2002) Patterning the vertebrate heart. Nature Reviews Genetics, 3 (7), 544-556.

(4) Hamburger, V. and Hamilton, H.L. (1992) A series of normal stages in the development of the chick embryo. Developmental Dynamics, 195 (4), 231-272.

(5) Sissman, N.J. (1970) Developmental landmarks in cardiac morphogenesis: comparative chronology. The American Journal of Cardiology, 25 (2), 141-148.

(6) Vincent, S.D. and Buckingham, M.E. (2010) How to make a heart: the origin and regulation of cardiac progenitor cells. Current Topics in Developmental Biology, 90, 1-41.

(7) Kelly, R.G. (2012) Chapter two - the second heart field, in Current Topics in Developmental Biology [Internet] (ed. B.G. Bruneau), Academic Press; pp. 33-65. Available from: http://www.sciencedirect.com/science/article/pii/B9780123877864000026 (last accessed March 6, 2014).

(8) Buckingham, M., Meilhac, S., and Zaffran, S. (2005) Building the mammalian heart from two sources of myocardial cells. Nature Reviews Genetics, 6 (11), 826-837.

(9) Arnold, S.J. and Robertson, E.J. (2009) Making a commitment: cell lineage allocation and axis patterning in the early mouse embryo. Nature Reviews Molecular Cell Biology, 10 (2), 91-103.

(10) Gilbert, S.F. (2010) Developmental Biology, 9th edn, Sinauer Associates, Inc., Sunderland, MA.

(11) Thiery, J.P., Acloque, H., Huang, R.Y.J., and Nieto, M.A. (2009) Epithelial-mesenchymal transitions in development and disease. Cell, 139 (5), 871-890.

(12) Ten Berge, D., Koole, W., Fuerer, C. et al. (2008) Wnt signaling mediates self-organization and axis formation in embryoid bodies. Cell Stem Cell, 3 (5), 508-518.

(13) Liu, P., Wakamiya, M., Shea, M.J. et al. (1999) Requirement for Wnt3 in vertebrate axis formation. Nature Genetics, 22 (4), 361-365.

(14) Takada, S., Stark, K.L., Shea, M.J. et al. (1994) Wnt-3a regulates somite and tailbud formation in the mouse embryo. Genes \& Development, 8 (2), 174-189.

(15) Yoshikawa, Y., Fujimori, T., McMahon, A.P., and Takada, S. (1997) Evidence that absence of Wnt-3a signaling promotes neuralization instead of paraxial mesoderm development in the mouse. Developmental Biology, 183 (2), 234-242.

(16) Kelly, O.G., Pinson, K.I., and Skarnes, W.C. (2004) The Wnt co-receptors Lrp5 and Lrp6 are essential for gastrulation in mice. Development (Cambridge, England), 131 (12), 2803-2815.

(17) Haegel, H., Larue, L., Ohsugi, M. et al. (1995) Lack of beta-catenin affects mouse development at gastrulation. Development (Cambridge, England), 121 (11), 3529-3537.

(18) Yamaguchi, T.P., Takada, S., Yoshikawa, Y. et al. (1999) T (Brachyury) is a direct target of Wnt3a during paraxial mesoderm specification. Genes \& Development, 13 (24), 3185-3190.

(19) Arnold, S.J., Stappert, J., Bauer, A. et al. (2000) Brachyury is a target gene of the Wnt//-catenin signaling pathway. Mechanisms of Development, 91 (1-2), 249-258.

(20) Tam, P.P.L. and Loebel, D.A.F. (2007) Gene function in mouse embryogenesis: get set for gastrulation. Nature Reviews Genetics, 8 (5), 368-381.

(21) Sun, X., Meyers, E.N., Lewandoski, M., and Martin, G.R. (1999) Targeted disruption of Fgf8 causes failure of cell migration in the gastrulating mouse embryo. Genes \& Development, 13 (14), 1834-1846.

(22) Yamaguchi, T.P., Harpal, K., Henkemeyer, M., and Rossant, J. (1994) fgfr-1 is required for embryonic growth and mesodermal patterning during mouse gastrulation. Genes \& Development, 8 (24), 3032-3044.

(23) Ciruna, B. and Rossant, J. (2001) FGF signaling regulates mesoderm cell fate specification and morphogenetic movement at the primitive streak. Developmental Cell, 1 (1), 37-49. 
(24) Bondue, A. and Blanpain, C. (2010) Mesp1 a key regulator of cardiovascular lineage commitment. Circulation Research, 107 (12), 1414-1427.

(25) Saga, Y., Hata, N., Kobayashi, S. et al. (1996) MesP1: a novel basic helix-loop-helix protein expressed in the nascent mesodermal cells during mouse gastrulation. Development (Cambridge, England), 122 (9), 2769-2778.

(26) Bondue, A., Lapouge, G., Paulissen, C. et al. (2008) Mesp1 acts as a master regulator of multipotent cardiovascular progenitor specification. Cell Stem Cell, 3 (1), 69-84.

(27) Lemonnier, M. and Buckingham, M.E. (2004) Characterization of a cardiac-specific enhancer, which directs $\alpha$-cardiac actin gene transcription in the mouse adult heart. The Journal of Biological Chemistry, 279 (53), 55651-55658.

(28) Evans, S.M., Yelon, D., Conlon, F.L., and Kirby, M.L. (2010) Myocardial lineage development. Circulation Research, 107 (12), 1428-1444.

(29) Plageman, T.F. and Yutzey, K.E. (2005) T-box genes and heart development: putting the "T" in heart. Developmental Dynamics, 232 (1), 11-20.

(30) Stennard, F.A. and Harvey, R.P. (2005) T-box transcription factors and their roles in regulatory hierarchies in the developing heart. Development (Cambridge, England), 132 (22), 4897-4910.

(31) Cai, C.-L., Zhou, W., Yang, L. et al. (2005) T-box genes coordinate regional rates of proliferation and regional specification during cardiogenesis. Development (Cambridge, England), 132 (10), $2475-2487$.

(32) Cai, C.-L., Liang, X., Shi, Y. et al. (2003) Isl1 identifies a cardiac progenitor population that proliferates prior to differentiation and contributes a majority of cells to the heart. Developmental Cell, 5 (6), 877-889.

(33) Dodou, E., Verzi, M.P., Anderson, J.P. et al. (2004) Mef2c is a direct transcriptional target of ISL1 and GATA factors in the anterior heart field during mouse embryonic development. Development (Cambridge, England), 131 (16), 3931-3942.

(34) Prall, O.W.J., Menon, M.K., Solloway, M.J. et al. (2007) An Nkx2-5/Bmp2/Smad1 negative feedback loop controls heart progenitor specification and proliferation. Cell, 128 (5), 947-959.

(35) Seo, S. and Kume, T. (2006) Forkhead transcription factors, Foxc1 and Foxc2, are required for the morphogenesis of the cardiac outflow tract. Developmental Biology, 296 (2), 421-436.

(36) Von Both, I., Silvestri, C., Erdemir, T. et al. (2004) Foxh1 is essential for development of the anterior heart field. Developmental Cell, 7 (3), 331-345.

(37) Lin, Q., Schwarz, J., Bucana, C., and Olson, E.N. (1997) Control of mouse cardiac morphogenesis and myogenesis by transcription factor MEF2C. Science, 276 (5317), 1404-1407.

(38) Tessari, A., Pietrobon, M., Notte, A. et al. (2008) Myocardial Pitx2 differentially regulates the left atrial identity and ventricular asymmetric remodeling programs. Circulation Research, 102 (7), 813-822.

(39) Schubert, W., Yang, X.Y., Yang, T.T.C. et al. (2003) Requirement of transcription factor NFAT in developing atrial myocardium. The Journal of Cell Biology, 161 (5), 861-874.

(40) Kokubo, H., Tomita-Miyagawa, S., Hamada, Y. and Saga, Y. (2007) Hesr1 and Hesr2 regulate atrioventricular boundary formation in the developing heart through the repression of $\mathrm{Tbx} 2$. Development (Cambridge, England), 134 (4), 747-755.

(41) Wu, S., Cheng, C.-M., Lanz, R.B. et al. (2013) Atrial identity is determined by a COUP-TFII regulatory network. Developmental Cell, 25 (4), 417-426.

(42) Brade, T., Männer, J., and Kühl, M. (2006) The role of Wnt signalling in cardiac development and tissue remodelling in the mature heart. Cardiovascular Research, 72 (2), 198-209.

(43) Lin, L., Cui, L., Zhou, W. et al. (2007) $\beta$-catenin directly regulates Islet1 expression in cardiovascular progenitors and is required for multiple aspects of cardiogenesis. Proceedings of the National Academy of Sciences of the United States of America, 104 (22), 9313-9318.

(44) Lough, J. and Sugi, Y. (2000) Endoderm and heart development. Developmental Dynamics, 217 (4), 327-342.

(45) Liberatore, C.M., Searcy-Schrick, R.D., Vincent, E.B., and Yutzey, K.E. (2002) Nkx-2.5 gene induction in mice is mediated by a Smad consensus regulatory region. Developmental Biology, 244 (2), 243-256.

(46) Van Wijk, B., Moorman, A.F.M., and van den Hoff, M.J.B. (2007) Role of bone morphogenetic proteins in cardiac differentiation. Cardiovascular Research, 74 (2), 244-255. 
(47) Hildreth, V., Webb, S., Chaudhry, B. et al. (2009) Left cardiac isomerism in the Sonic hedgehog null mouse. Journal of Anatomy, 214 (6), 894-904.

(48) Hoffmann, A.D., Peterson, M.A., Friedland-Little, J.M. et al. (2009) Sonic hedgehog is required in pulmonary endoderm for atrial septation. Development (Cambridge, England), 136 (10), 1761-1770.

(49) Kruithof, B.P.T., van Wijk, B., Somi, S. et al. (2006) BMP and FGF regulate the differentiation of multipotential pericardial mesoderm into the myocardial or epicardial lineage. Developmental Biology, 295 (2), 507-522.

(50) van Wijk, B., van den Berg, G., Abu-Issa, R. et al. (2009) Epicardium and myocardium separate from a common precursor pool by crosstalk between bone morphogenetic protein-and fibroblast growth factor-signaling pathways. Circulation Research, 105 (5), 431-441.

(51) Rhinn, M. and Dollé, P. (2012) Retinoic acid signalling during development. Development (Cambridge, England), 139 (5), 843-858.

(52) Loh, Y.-H., Wu, Q., Chew, J.-L. et al. (2006) The Oct4 and Nanog transcription network regulates pluripotency in mouse embryonic stem cells. Nature Genetics, 38 (4), 431-440.

(53) Boyer, L.A., Lee, T.I., Cole, M.F. et al. (2005) Core transcriptional regulatory circuitry in human embryonic stem cells. Cell, 122 (6), 947-956.

(54) Takahashi, K. and Yamanaka, S. (2006) Induction of pluripotent stem cells from mouse embryonic and adult fibroblast cultures by defined factors. Cell, 126 (4), 663-676.

(55) Takahashi, K., Tanabe, K., Ohnuki, M. et al. (2007) Induction of pluripotent stem cells from adult human fibroblasts by defined factors. Cell, 131 (5), 861-872.

(56) Yu, J., Vodyanik, M.A., Smuga-Otto, K. et al. (2007) Induced pluripotent stem cell lines derived from human somatic cells. Science, 318 (5858), 1917-1920.

(57) Boulting, G.L., Kiskinis, E., Croft, G.F. et al. (2011) A functionally characterized test set of human induced pluripotent stem cells. Nature Biotechnology, 29 (3), 279-286.

(58) Esteban, M.A., Wang, T., Qin, B. et al. (2010) Vitamin C enhances the generation of mouse and human induced pluripotent stem cells. Cell Stem Cell, 6 (1), 71-79.

(59) Liang, G., Taranova, O., Xia, K., and Zhang, Y. (2010) Butyrate promotes induced pluripotent stem cell generation. The Journal of Biological Chemistry, 285 (33), 25516-25521.

(60) Chen, G., Gulbranson, D.R., Hou, Z. et al. (2011) Chemically defined conditions for human iPSC derivation and culture. Nature Methods, 8 (5), 424-429.

(61) Jia, F., Wilson, K.D., Sun, N. et al. (2010) A nonviral minicircle vector for deriving human iPS cells. Nature Methods, 7 (3), 197-199.

(62) Yamanaka, S. and Blau, H.M. (2010) Nuclear reprogramming to a pluripotent state by three approaches. Nature, 465 (7299), 704-712.

(63) Okita, K., Matsumura, Y., Sato, Y. et al. (2011) A more efficient method to generate integrationfree human iPS cells. Nature Methods, 8 (5), 409-412.

(64) Rais, Y., Zviran, A., Geula, S. et al. (2013) Deterministic direct reprogramming of somatic cells to pluripotency. Nature, $\mathbf{5 0 2}$ (7469), 65-70.

(65) Ojala, M., Rajala, K., Pekkanen-Mattila, M. et al. (2012) Culture conditions affect cardiac differentiation potential of human pluripotent stem cells. PLoS One, 7 (10), e48659.

(66) Niwa, H., Burdon, T., Chambers, I., and Smith, A. (1998) Self-renewal of pluripotent embryonic stem cells is mediated via activation of STAT3. Genes \& Development, 12 (13), 20482060.

(67) Vallier, L., Alexander, M., and Pedersen, R.A. (2005) Activin/Nodal and FGF pathways cooperate to maintain pluripotency of human embryonic stem cells. Journal of Cell Science, 118 (19), 4495-4509.

(68) Ludwig, T.E., Bergendahl, V., Levenstein, M.E. et al. (2006) Feeder-independent culture of human embryonic stem cells. Nature Methods, 3 (8), 637-646.

(69) Mummery, C.L., Zhang, J., Ng, E.S. et al. (2012) Differentiation of human embryonic stem cells and induced pluripotent stem cells to cardiomyocytes a methods overview. Circulation Research, 111 (3), 344-358.

(70) Burridge, P.W., Keller, G., Gold, J.D., and Wu, J.C. (2012) Production of de novo cardiomyocytes: human pluripotent stem cell differentiation and direct reprogramming. Cell Stem Cell, 10 (1), 16-28. 
(71) Schulz, H., Kolde, R., Adler, P. et al. (2009) The FunGenES database: a genomics resource for mouse embryonic stem cell differentiation. PLoS One, 4 (9), e6804.

(72) Kattman, S.J., Huber, T.L., and Keller, G.M. (2006) Multipotent Flk-1 + cardiovascular progenitor cells give rise to the cardiomyocyte, endothelial, and vascular smooth muscle lineages. Developmental Cell, 11 (5), 723-732.

(73) Kattman, S.J., Adler, E.D., and Keller, G.M. (2007) Specification of multipotential cardiovascular progenitor cells during embryonic stem cell differentiation and embryonic development. Trends in Cardiovascular Medicine, 17 (7), 240-246.

(74) Vliet, P.V., Wu, S.M., Zaffran, S., and Pucéat, M. (2012) Early cardiac development: a view from stem cells to embryos. Cardiovascular Research, 96 (3), 352-362.

(75) Rai, M., Walthall, J., Hu, J., and Hatzopoulos, A.K. (2011) Continuous antagonism by Dkk1 counter activates canonical wnt signaling and promotes cardiomyocyte differentiation of embryonic stem cells. Vanderbilt University.

(76) Martin-Puig, S., Wang, Z., and Chien, K.R. (2008) Lives of a heart cell: tracing the origins of cardiac progenitors. Cell Stem Cell, 2, 320-331.

(77) Fuegemann, C.J., Samraj, A.K., Walsh, S. et al. (2012) Differentiation of Mouse Embryonic Stem Cells into Cardiomyocytes via the Hanging-Drop and Mass Culture Methods. Available from: http://onlinelibrary.wiley.com/doi/10.1002/9780470151808.sc01f11s15/abstract (last accessed March 6, 2014).

(78) Burridge, P.W., Thompson, S., Millrod, M.A. et al. (2011) A universal system for highly efficient cardiac differentiation of human induced pluripotent stem cells that eliminates interline variability. PLoS One, 6 (4), e18293.

(79) Evans, M.J. and Kaufman, M.H. (1981) Establishment in culture of pluripotential cells from mouse embryos. Nature, 292 (5819), 154-156.

(80) Laflamme, M.A., Chen, K.Y., Naumova, A.V. et al. (2007) Cardiomyocytes derived from human embryonic stem cells in pro-survival factors enhance function of infarcted rat hearts. Nature Biotechnology, 25 (9), 1015-1024.

(81) Shim, J., Grosberg, A., Nawroth, J.C. et al. (2012) Modeling of cardiac muscle thin films: pre-stretch, passive and active behavior. Journal of Biomechanics, 45 (5), 832-841.

(82) Maltsev, V., Wobus, A., Rohwedel, J. et al. (1994) Cardiomyocytes differentiated in vitro from embryonic stem cells developmentally express cardiac-specific genes and ionic currents. Circulation Research, 75 (2), 233-244.

(83) Mummery, C., Ward, D., van den Brink, C. et al. (2002) Cardiomyocyte differentiation of mouse and human embryonic stem cells. Journal of Anatomy, 200 (3), 233-242.

(84) Zwi, L., Caspi, O., Arbel, G. et al. (2009) Cardiomyocyte differentiation of human induced pluripotent stem cells. Circulation, 120 (15), 1513-1523.

(85) Kattman, S.J., Witty, A.D., Gagliardi, M. et al. (2011) Stage-specific optimization of activin/nodal and BMP signaling promotes cardiac differentiation of mouse and human pluripotent stem cell lines. Cell Stem Cell, 8 (2), 228-240.

(86) Yang, L., Soonpaa, M.H., Adler, E.D. et al. (2008) Human cardiovascular progenitor cells develop from a KDR + embryonic-stem-cell-derived population. Nature, 453 (7194), 524-528.

(87) Cao, N., Liu, Z., Chen, Z. et al. (2012) Ascorbic acid enhances the cardiac differentiation of induced pluripotent stem cells through promoting the proliferation of cardiac progenitor cells. Cell Research, 22 (1), 219-236.

(88) Wu, X., Ding, S., Ding, Q. et al. (2004) Small molecules that induce cardiomyogenesis in embryonic stem cells. Journal of the American Chemical Society, 126 (6), 1590-1591.

(89) Elliott, D.A., Braam, S.R., Koutsis, K. et al. (2011) NKX2-5eGFP/w hESCs for isolation of human cardiac progenitors and cardiomyocytes. Nature Methods, 8 (12), 1037-1040.

(90) Zhang, J., Wilson, G.F., Soerens, A.G. et al. (2009) Functional cardiomyocytes derived from human induced pluripotent stem cells. Circulation Research, 104 (4), e30-41.

(91) Zhang, Q., Jiang, J., Han, P. et al. (2011) Direct differentiation of atrial and ventricular myocytes from human embryonic stem cells by alternating retinoid signals. Cell Research, 21 (4), 579 587. 
(92) Kolossov, E., Lu, Z., Drobinskaya, I. et al. (2005) Identification and characterization of embryonic stem cell-derived pacemaker and atrial cardiomyocytes. The FASEB Journal, 19 (6), 577 579.

(93) Wiese, C., Nikolova, T., Zahanich, I. et al. (2011) Differentiation induction of mouse embryonic stem cells into sinus node-like cells by suramin. International Journal of Cardiology, 147 (1), 95-111.

(94) Itzhaki, I., Maizels, L., Huber, I. et al. (2011) Modelling the long QT syndrome with induced pluripotent stem cells. Nature, 471 (7337), 225-229.

(95) Wilke, R.A., Lin, D.W., Roden, D.M. et al. (2007) Identifying genetic risk factors for serious adverse drug reactions: current progress and challenges. Nature Reviews Drug Discovery, 6 (11), 904-916. 


\title{
3
}

\section{Chemical Genetics in Cardiomyocyte Generation}

\author{
Daqing $\mathrm{Jin}^{1}$, Qiao $\mathrm{Li}^{1}$, and Tao P. Zhong ${ }^{2}$ \\ ${ }^{I}$ Department of Genetics, Fudan University School of Life Sciences, China \\ ${ }^{2}$ State Key Laboratory of Genetic Engineering, Fudan University School of Life Sciences, \\ China; Department of Medicine, Vanderbilt University, USA
}

\subsection{Introduction}

Myocardial infarction and ischemic heart disease are caused by an acute reduction of blood to the myocardium, which has the direct effect of diminishing oxygen supply. This results in a loss of large numbers of cardiomyocytes, leading to hypertrophy and heart failure. Care of such patients places enormous burdens on the health care system, requiring 1 million hospitalizations every year at an annual cost of $\$ 35$ billion in the USA [1]. Development of therapeutics that can facilitate new cardiomyocyte generation would be of enormous medical and economic impact.

One option for replenishing a depleted cardiomyocyte population is transplantation of exogenous cells. Over the past decade, many cell types have been evaluated in order to find the best source for cardiac cell therapy. A wide variety of stem cells, including bone marrow mononuclear cells (BMCs), mesenchymal stem cells (MSCs), cardiac stem cells (CSCs), and embryonic stem cells (ESCs), have been examined regarding their capacity to repair the infarcted myocardium and improve functionally the diseased heart $[1,2]$. These stem cells have been transplanted in animal models of myocardial infarction and have been demonstrated to reduce infarct size, attenuate left-ventricle remodeling, increase vascular

Chemical Biology in Regenerative Medicine: Bridging Stem Cells and Future Therapies, First Edition. Edited by Charles C. Hong, Ada S. Ao, and Jijun Hao.

(C) 2014 John Wiley \& Sons, Ltd. Published 2014 by John Wiley \& Sons, Ltd. 
density, and improve myocardial function $[1,2]$. However, a recent clinical trial to test BMC therapy in patients with ischemic heart failure revealed no beneficial effects [3], raising considerable concerns that BMCs and other exogenous stem cells may have low capacities to integrate and differentiate into cardiomyocytes in the patient heart. Other trials are underway to examine the potential clinical effects of c-Kit-positive and cardiospherederived stem cells for the treatment of acute myocardial infarction. These findings highlight the importance and urgency of developing alternative therapeutic approaches.

\section{2 iPSC Generation}

The advent of induced pluripotent stem cells (iPSCs) has offered a possible solution in terms of generating functional cardiomyocytes. Somatic cells can be reprogrammed into an embryonic-like state by transferring nuclear contents into oocytes [4] or by fusion with embryonic stem cells (ESCs) [5,6]. In 2006, Yamanaka et al. hypothesized that factors that play essential roles in the maintenance of ESC identity may also play pivotal roles in the induction of pluripotency in differentiated cells [7]. They developed a clever screen in which induction of the pluripotent cell state could be selected by the expression of a neomycin/lacZ fusion gene ( $\beta$ geo). The $\beta$ geo cassette was inserted into the Fbx15 locus in the genome of mouse embryonic fibroblasts. Fbx15 can be specifically expressed in mouse ESCs and is indispensable for the maintenance of ESC pluripotency and mouse development. In the pluripotent stem cells, the Fbx15 promoter activates $\beta$ geo expression, resulting in G418 resistance. The initially selected candidate genes were pooled together and delivered to fibroblasts by retroviral transfection to test for reprogramming. Successful combinations of factors capable of G18 resistance were selected. This pool was then narrowed down by reducing factors to Oct4, Klf4, Sox2, and c-Myc (OKSM). iPSCs generate various cell types in teratoma assays and can contribute to tissues in chimeric mice after transplantation into mouse blastocysts. Human pluripotent stem cells (hPSCs) can also be generated from fibroblasts expressing OKSM or slightly different combinations, including Nanog, Lin28, Sox2, and Oct4 $[8,9]$.

Recently, it was demonstrated that OKSM factors initially interact with the genome during cell reprogramming in the first 48 hours [10]. These factors extensively access distal regulatory elements and activate transcription of many genes required for reprogramming. In both undifferentiated stem cells and fully differentiated cells, genes can exist in stable states in which they are transcriptionally silent. During initial reprogramming, a subset of transcription factors termed "pioneer factors" initially access closed chromatin and directly make it competent to be bound by other factors [10]. Oct4, Sox2, and Klf4 together bind as pioneer factors to closed chromatin sites, while c-Myc facilitates the binding of OSK to chromatin. Many of the genes that promote cell reprogramming reside in the closed chromatin regions of the genome. Megabase-scale chromatin domains are spanned by H3K9me3. Knockdown of relevant histone methyltransferases allows OSKM binding and enhances cell reprogramming. H3K9me3-containing regions of the genome are refractory to initial OSKM binding and thereby impede the efficiency of reprogramming [10]. Investigating the initial binding of OSKM to the fibroblast genome helps with understanding the mechanisms of reprogramming, leading to potential therapeutic applications. 


\subsection{The Chemical Genetics Approach in iPSC Generation}

The iPSC technique represents a breakthrough in the stem cell field and offers new perspectives on generating functional cardiomyocytes, but it comes with safety issues. For example, use of iPSCs is hindered by the potential risks of genetic mutations caused by the integration of exogenous genetic material into chromosomes. Although several nonintegrative methods (e.g. episomal plasmid, protein, and mRNA transfection) have been developed to generate iPSCs, induction efficiency is still quite low [11]. In contrast, small molecules provide several distinct advantages in controlling protein functions (e.g. temporal control, reversibility, tunability, modularity, and tractability). Hence they have attracted much interest in relation to steering reprogramming toward a more efficient and safe process [11].

To date, various strategies have been developed to generate iPSCs with fewer or no exogenous genetic manipulations. Different combinations of chemical compounds have been identified to replace some exogenous transcription factors and enhance the efficiency of reprogramming. Recent reports indicate that reprogramming efficiency can be enhanced by the presence of small molecules, such as valproic acid (VPA, a histone deacetylase inhibitor), AZA (a DNA methyltransferase inhibitor), butyrate (a histone deacetylase (HDAC) inhibitor), and vitamin C (Figure 3.1) [12-15].

Vitamin $\mathrm{C}$ improves iPSC generation mainly by reducing p53 levels and alleviating cell senescence while still maintaining an intact DNA repair machinery (basal levels of p53) [14]. It is possible that it accelerates transcriptome changes during reprogramming in other ways as well. Vitamin $\mathrm{C}$ is a cofactor in reactions driven by dioxygenases, including collagen prolyl hydroxylases, hypoxia-inducible factor, and histone demethylases. During reprogramming, vitamin $\mathrm{C}$ may increase the activity of these enzymes, promote epigenetic modifications, and enhance iPSC generation. For example, vitamin $\mathrm{C}$ may allow the reprogramming to run smoothly by activating histone demethylases, which are important for development and modulate the expression of the ESC master transcription factor Nanog [16]. It is tempting to speculate that vitamin $C$, as a compelling antioxidant, might improve the efficiency of reprogramming by suppressing reactive oxygen species (ROS) produced by cell metabolism [14].

VPA, an HDAC inhibitor, can increase reprogramming efficiency and enable efficient induction of iPSCs without introducing the oncogene c-Myc, suggesting that chromatin modification is a key step in reprogramming fibroblasts to pluripotent cells [12]. Likewise, butyrate, a small-chain fatty acid, acts as HDAC inhibitor and promotes protein acetylation at targets such as H3K9 [15]. Simultaneously, it also accelerates promoter DNA demethylation and expression of endogenous pluripotency-associated genes such as POU5F1/OCT4 and DPPA2. Furthermore, butyrate may possibly stimulate reprogramming by modulating the activities of nonhistone key regulators, as it exhibits diverse cellular effects in culture, including cell-cycle arrest and induction of protein synthesis [17]. AZA (5-aza-cytidine), acting as an inhibitor of DNA methyltransferase (DNMT), improves the overall reprogramming efficiency, indicating that epigenetic remodeling is a key step in the reprogramming process [13].

Furthermore, the small molecules SB431542 and PD0325901, acting as inhibitors of transforming growth factor beta (TGF $\beta$ ) and MAPK/ERK pathways, respectively, significantly enhance the reprogramming efficiency of human fibroblasts through the expression of the four transcriptional factors (Figure 3.1) [18]. TGF $\beta$ is a prototypical cytokine for the 


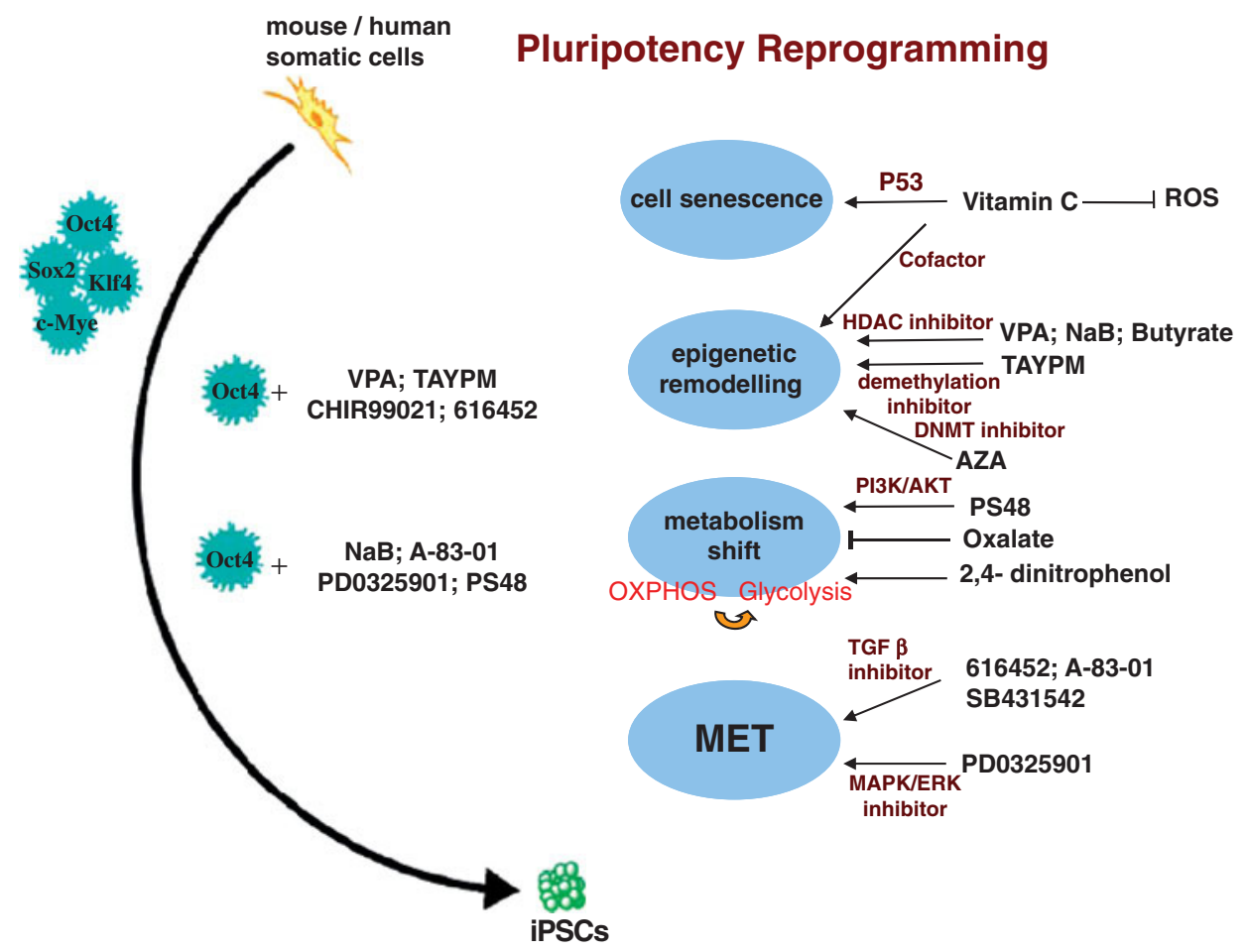

Figure 3.1 During iPSC reprogramming, a variety of small-molecule compounds have been identified as enhancing pluripotent reprogramming efficiency in the presence of four transcriptional factors (OSKM) or Oct4 through cell senescence, epigenetic remodeling, metabolism shift, and mesenchymal-epithelial transition (MET)

induction of epithelial-mesenchymal transition (EMT) and the maintenance of the mesenchymal state [19]. MAPK/ERK signaling facilitates EMT [20] and occurs downstream of TGF $\beta$ [21]. The positive effects of SB431542 and PD0325901 on reprogramming indicate that TGF $\beta$ and MAPK/ERK pathway antagonists have a direct impact on the reprogramming process, mainly by promoting the mesenchymal-epithelial transition (MET).

Ding's group was the first to report that a small-molecule combination - the G9a histone methyltransferase inhibitor BIX-01294 (BIX) and the L-type calcium channel agonist Bayk8644 (Bayk) - can enable mouse embryonic fibroblasts (MEFs) to reprogram into iPSCs through the transduction of two factors (Oct4 and Klf4) [22]. BIX treatment causes histone modification at the epigenetic level and thus enhances reprogramming activity. Bayk works synergistically with BIX to further increase reprogramming efficiency and has no impact on reprogramming in the absence of BIX. Bayk might influence the reprogramming process in specific manners rather than at general epigenetic levels [22].

Both mouse and human iPSCs can be generated by ectopic expression of Oct 4 plus different combinations of small molecules. A defined small-molecule cocktail, including the histone deacetylase inhibitor NaB, the TGF-G receptor inhibitor A-83-01, the MAPK/ERK inhibitor PD0325901, and PS48 (an activator of 3-phosphoinositide-dependent kinase-1), 
is sufficient to reprogram human primary somatic cells to iPSCs through the expression of a single transcription factor, Oct4 (Figure 3.1) [23]. It is known that many types of stem cell, including pluripotent stem cells, mainly rely on glycolysis followed by lactic acid fermentation in the cytosol to produce energy [23]. This is in contrast to most differentiated cells, in which oxidation of pyruvate in mitochondria is used to produce energy. This might be advantageous for stem cells, as glycolytic metabolism effectively produces various macromolecular precursors in order to meet metabolic and energy demands, while generating fewer ROS (which induce oxidative damage). Consistent with the hypothesis that PS48 facilitates a metabolic conversion from mitochondrial oxidation to glycolysis during the reprogramming process, treatment with PS48 activates downstream AKT/PKB, upregulates expression of several key glycolytic genes, and consequently enhances glycolysis, as measured by increased lactate production. Notably, several known small molecules that have been widely used to modulate mitochondrial oxidation, glycolytic metabolism, or hypoxia-inducible factor (HIF) pathway activation also show corresponding effects on reprogramming. For example, compounds that promote glycolytic metabolism (such as 2,4-dinitrophenol and N-oxaloylglycine) enhance reprogramming, whereas compounds that block glycolytic metabolism (such as oxalate) inhibit reprogramming (Figure 3.1) [23-25].

A study from Li et al. showed that the combination of four small molecules, VPA (an HDAC inhibitor), CHIR99021 (a glycogen synthase kinase $3 \beta$ (GSK3 $\beta$ ) inhibitor), 616452 (a TGF $\beta$ signaling inhibitor), and tranylcypromine (TAYPM, an H3K4 demethylation inhibitor) (referred to collectively as VC6T), was sufficient to generate iPSCs from mouse fibroblasts with a single transcription factor, Oct4, thus replacing Sox2, Klf4, and c-Myc (Figure 3.1) [26]. Based on previous studies, Li et al. concluded that VC6T may facilitate the reprogramming process by lowering four major barriers (two epigenetic barriers and two signaling barriers). The effects of VPA and tranylcypromine suggest that H3K4 demethylation and histone deacetylation are two critical epigenetic barriers to reprogramming that may repress the establishment of a pluripotency transcriptional network.

In embryonic stem cells, it has been revealed that Tcf3, one of the key transcriptional regulators downstream of the Wnt pathway, occupies and regulates the promoters of Oct4, Sox2, and Nanog [27,28]. In MEFs, these endogenous pluripotency transcription factors are silenced. During reprogramming, Wnt signaling can directly potentiate the effect of exogenous Oct4, Sox2, and Klf4, as it does in ESCs [27], thereby directly promoting the induction of pluripotency in the absence of c-Myc transduction. Inhibition of GSK3 $\beta$ to activate Wnt signaling could enhance mESC self-renewal and cell reprogramming, possibly by regulating the stability of the c-Myc protein $[29,30]$. As is known, the MET is a crucial initiating event in the derivation of iPSCs from fibroblasts $[31,32]$. TGF $\beta$ induces EMT by both Smad-dependent and Smad-independent signaling events. Recent studies demonstrate that full reversal of EMT morphology and patterns of gene expression can be accomplished by concurrently inhibiting T $\beta$ RI (TGF $\beta$ type I receptor) kinase. Besides promoting MET [32-34], TGF $\beta$ inhibition has also been reported to facilitate Nanog gene expression [35]. Together, these findings indicate that GSK3 $\beta$ and TGF $\beta$ signaling may be two major signaling barriers that normally repress the reprogramming process.

Inspired by the approach to iPSC generation using multiple transcription factors (TFs), researchers have devised an alternative method for directly reprogramming mouse fibroblasts into cardiomyocytes [36]. They employ the transient overexpression of Oct4, Sox2, 
and Klf4 to activate early cardiac programming in as little as 4 days and then exposed cells to leukemia inhibitory factor (LIF)-free medium containing $15 \%$ fetal bovine serum (FBS) and 5\% knockout serum replacer (KSR) for 6 days, before switching to $1 \% \mathrm{FBS}$ and $14 \%$ KSR for 3 days. During the initial 9-day period, a small-molecule Janus kinase (JAK) inhibitor, JI1, is continuously kept in the culture condition to promote cardiomyogenesis and prevent iPSC intermediates from forming. From day 9 onwards, the cells are cultured in chemically defined media, with bone morphogenetic protein 4 (BMP4) treatment for the first 5 days. This process generated a large number of spontaneously contracting patches of cardiac cells in as few as 11 days, as well as minor iPSC-like cells. Remarkably, nearly $40 \%$ of cells had become mature $(\mathrm{cTnT}+)$ cardiac populations by day 18 . The authors attributed the increased efficiency to the generation of highly proliferative progenitor cells, and further speculated that these less-mature cardiac cells may be more valuable for heart regeneration than terminally differentiated cardiomyocytes. This method potentially offers a faster and more efficient route for generation of desired cell types. Once the safety issue is essentially eliminated, it will be more attractive for eventual therapeutic use.

\subsection{Heart Regeneration}

Developing treatments that can stimulate endogenous cardiomyocyte regeneration in areas of infarction could be the new frontier of regenerative therapy. An ideal therapeutic option would be to employ small molecules that have the capacity to stimulate and accelerate endogenous cardiomyocyte regeneration after injury. In zebrafish, adult heart regenerates after partial surgical resection [37]. Approximately 3\% of cardiomyocytes in the compact myocardium of adult zebrafish hearts incorporated the thymidine analog bromodeoxyuridine (BrdU) during a 7-day pulse-labeling experiment. Two weeks after amputation of the cardiac apex, the fraction of BrdU-positive cardiomyocytes had increased by 10-fold, and this parameter remained as high as $20 \% 1$ month after injury. By 60 days post-amputation, the lost cardiomyocytes had completely regenerated. Cardiac muscle supplanted the fibrin clot without generating scar tissue, leading to restoration of vascularized cardiac muscle and normal electrical coupling with adjacent cells. Another three dissimilar injury models - genetic ablation [38], cryocauterization [39], and hypoxia/reoxygenation [40] also stimulated robust myocardial regeneration in zebrafish. The injured heart had entirely regenerated after 30,130, and 30 days, respectively. Organ-wide heart tissue is responsive to injury during zebrafish heart regeneration, not just that near the trauma. All major cardiac tissues, including epicardium, endocardium, and myocardium, appear to employ this strategy, in which endocardial cells show the earliest responses [41]. Over different time courses, depending on the cell type, these injury-activated expression signatures disappear globally and seem to localize to the injury site. Factors released during cardiac inflammation may help to trigger an organ-wide injury response, which might be a key to regenerative success. Signaling pathways that participate in zebrafish heart regeneration have been identified, including fibroblast growth factor (FGF) [42], platelet-derived growth factor (PDGF) [43], and retinoic acid (RA) pathways [44]. In mammals, the heart of 1-dayold neonatal mice can regenerate after partial surgical resection or left anterior descending artery (LAD) ligation $[45,46]$. Local injury induces global cardiomyocyte morphology changes and proliferation in the neonatal mouse heart. This regenerative capacity is lost 
7 days after birth, when resection or ligation surgery lead to the formation of a fibrotic scar. The timeline coincides with the withdrawal of cardiomyocytes from the cell cycle through the completion of mitosis without cytokinesis, resulting in formation of binucleated cells. This postnatal switch in cardiomyocyte proliferation leads to the transient presence of regenerative capacity in the neonatal mouse heart. Scar formation may also cause loss of regeneration capacity by 7 days. In humans, over the course of an average lifetime almost $45 \%$ of cardiomyocytes slowly renew into adulthood, while $55 \%$ remain after birth [47]. This turnover is very low and decreases significantly with age. Both the integration of radioactive ${ }^{14} \mathrm{C}$ (generated by nuclear bomb tests during the Cold War) into DNA and the infusion of iododeoxyuridine (IdU, a thymidine analog used as a radiosensitizer for therapy [48]) provide evidence for this measurable ability in the adult human heart. Compared with lower vertebrate heart, mammalian heart has more fibroblasts and less endocardial cells; more importantly, mammalian cardiomyocytes contain more myofibrils and have a higher DNA content (binucleated cells with diploid nucleus in rodents, mononucleated cells with polyploid nucleus in humans). These cardiac cell properties result in poor regenerative capacity when the mammalian heart is injured.

Inducible genetic fate-mapping analyses demonstrate that zebrafish heart regeneration occurs through cardiomyocyte dedifferentiation and proliferation $[49,50]$. The 4hydroxytamoxifen (4-HT)-inducible Cre/lox system in zebrafish has been established to prelabel cardiomyocytes with enhanced green fluorescent protein (EGFP) prior to regeneration tests. After amputation of the apex, the new apical myocardium is $100 \% \mathrm{EGFP}^{+}$, indicating that new cardiomyocytes arise from existing cardiomyocytes rather than the recruitment of cardiomyogenic precursors. A subpopulation of cardiomyocytes within the ventricular wall expresses Gata4 to activate an embryonic program that facilitates dedifferentiation and proliferation and contributes substantially to local muscle regeneration. This is consistent with the results of a cardiac sarcomere study, indicating that proliferating cardiomyocytes detach from one another and acquire a less organized sarcomeric structure during regeneration. Likewise, recent studies suggest that mammalian cardiomyocytes regenerate through the division of preexisting cardiomyocytes [51]. By combining genetic fate-mapping with stable isotope-labeling, the nonradioactive tracer $\left[{ }^{15} \mathrm{~N}\right]$ thymidine and DNA synthesis can be measured using multi-isotope imaging mass spectrometry (MIMS) in the adult heart of double-transgenic MerCreMer/ZEG mice. During normal mammalian myocardial homeostasis, mature resident cardiomyocytes are the dominant source of cardiomyocyte replacement, at a rate of about $0.76 \%$ per year. This process is increased fivefold during recovery from myocardial infarction. Adult cardiomyocytes retain some capacity to re-enter the cell cycle, but after injury most DNA synthesis occurs in preexisting cardiomyocytes, without completion of cell division. In contrast, cardiac progenitor cells do not make a significant contribution to myocardial homeostasis in mammals, and their roles after injury are also limited. However, when bone marrow-derived c-Kit + cells are injected into the infarcted heart, the transplanted cells stimulate cardiomyocyte regeneration some degrees removed from endogenous progenitors or unlabeled cardiomyocytes through paracrine effects [52]. The epicardium and endocardium also appear to play indispensable signaling roles in heart regeneration. Some of the activated epicardial cells can transdifferentiate into cardiomyocytes at a low reprogramming rate while preconditioning with thymosin $\beta 4$ injections [53]. Together, these findings provide strong evidence that the adult human heart is capable of regenerating cardiomyocytes in order to preserve its 
function, although the cell turnover is low throughout life. An ideal therapeutic option would be to employ small molecules that have capacity to stimulate and accelerate endogenous cardiomyocyte regeneration after injury.

\subsection{The Chemical Genetics Approach in Heart Regeneration}

Although high-throughput, high-content small-molecule heart-regeneration screens present great challenges in adult zebrafish, they can be carried out in zebrafish embryos. Compounds identified from heart developmental screens may have capacity to induce heart regeneration, considering similar signaling molecules and pathways are employed.

A newly developed fluorescent ubiquitylation-based cell-cycle indicator (FUCCI) screening system employs two fusion proteins (mCherry-zCdt1 and Venus-hGeminin) that are expressed during the G1 and S/G2/M phases. The FUCCI system enables the visualization of cell-proliferation events in live animals. After the cmlc2:FUCCI embryos have been exposed to a panel of compounds targeting various developmental signaling pathways, small molecules that activate or block Hedgehog (Hh), insulin-like growth factor (IGF), and TGF $\beta$ signaling pathways can be seen to regulate cardiomyocyte proliferation [54]. Direct examination of zebrafish heart regeneration after mechanical or genetic ablation injuries indicates that Hh, IGF, and TGF $\beta$ signaling pathways are activated in regenerating cardiomyocytes. These pathways can be pharmacologically manipulated by molecules identified from heartdevelopment screens. Since the embryonic heart size primarily reflects cardiomyocyte generation, the size of fluorescence-expressing heart can also be used as a visual screen phenotype. Three structurally related compounds with the same pharmacophore, named cardionogen- 1 to -3 , have been found to enlarge heart size via myocardial hyperplasia [55]. The increased cardiomyocyte number in cardionogen-treated embryos is due to an expansion of the cardiac progenitor cell population, and it may also promote cardiac cell proliferation. Mechanistically, cardionogen functions as an inhibitor of the Wnt/ $\beta$-catenin signaling pathway and can rescue cardiomyocyte deficiency and heart-specific phenotypes induced by Wnt8 overexpression during development. Expanding the panel of pharmacological manipulations that regulate this process will help reveal mechanisms that induce myocardial regeneration. Compounds identified from the unbiased screens in zebrafish are likely to represent promising tools for probing and potentially enhancing cardiomyocyte proliferation in the injured mammalian heart.

Mammalian ESCs have also been used to screen small molecules in inducing cardiomyocyte formation. Among 550 known pathway modulators, small-molecule IWR1 (inhibitor of Wnt response) and IWP3 (inhibitor of Wnt production) were identified as inducing cardiogenesis, demonstrating that Wnt inhibition is sufficient to drive human ESCs to form cardiomyocytes [56]. However, treating zebrafish embryos with IWR1 results in truncation of the posterior axis and disruption of the cardiac chamber formation. Notably, cardionogen treatment induces cardiac cell formation without causing tail truncation and atrium disruption in zebrafish, indicating that cardionogen has little toxicity compared to IWR1. Consistent with the in vivo activities, cardionogen blocks Wnt/ $\beta$-catenin-dependent transcription in embryos and ESCs but not in HEK293 cells, while IWR1 blocks Wnt signaling in embryos, ESCs, and HEK293 cells. These findings suggest that cardionogen selectively reduces Wnt signaling associated with certain tissues, revealing the benefits of 
whole-embryo screens by targeting specific cell types. Whole-embryo screens offer considerable advantages in drug discovery by evaluating target cell populations and organs, as well as the relevant physiological context. The identified compounds normally have efficacy with little toxicity, which can be further developed as medicine.

\subsection{Cardiac Cell Transdifferentiation}

Recent studies have revealed an advanced reprogramming technology that can directly convert one somatic cell type to another, such as fibroblasts to cardiomyocytes, representing a new approach to generating cardiomyocytes from endogenous cells. In order to accomplish this goal, an assay system was developed in which the induction of mature cardiomyocytes from fibroblasts could be analyzed quantitatively by reporter-based fluorescence-activated cell sorting (FACS) [57]. In order to have sufficient cardiac fibroblasts for transdifferentiation, cells which express not GFP but rather Thy1 (a marker of cardiac fibroblasts) were obtained from neonatal aMHC-GFP hearts by explant culture [58]. Fourteen genes were selected, using microarray analyses between cardiomyocytes and cardiac fibroblasts, as candidate factors that induce fibroblasts into cardiomyocytes [58]. All these candidate genes were introduced into cardiac fibroblasts by retrovirus-mediated transfection. A combination of three transcription factors (Gata4, Mef2c, and Tbx5) was found to be sufficient to convert cardiac fibroblasts into functional induced cardiac-like myocytes (iCLMs). To exclude the possibility that iCLMs were derived from rare cardiac progenitors or contamination of cardiomyocytes, the potential for mouse tail-tip dermal fibroblasts to produce iCLMs was tested. Thy $1+/$ GFP - tail-tip dermal fibroblasts transduced with GMT can also be reprogrammed into cardiac-like myocytes [57].

To determine whether fibroblasts directly convert into cardiomyocytes or whether they undergo dedifferentiation before further differentiation, Isl1- and Mesp-1-yellow fluorescent protein (YFP) mice were obtained by crossing Isl1-Cre and Mesp-1-Cre mice with R26R-EYFP mice, respectively [58]. Is11 is a marker of cardiac progenitors that are transiently expressed before cardiac differentiation. Mesp-1 is the earliest pan-cardiovascular progenitor cell marker that is transiently expressed in nascent mesoderm before further cardiovascular differentiation. If iCLMs generated from fibroblasts undergo a cardiac progenitor cell or early cardiac mesodermal state, YFP will permanently express in their descendants. Isl1-YFP-/Thy1+ and Mesp-1-YFP-/Thy1+ fibroblast cells are isolated and transduced with GMT. The cardiac cells expressing cTnT do not express YFP, suggesting that the transdifferentiation does not involve the cardiac progenitor or cardiac mesoderm cell state but rather directly reprograms from fibroblasts into functional cardiomyocytes [57].

The human heart contains 30\% cardiomyocytes and 70\% cardiac fibroblasts. Cardiac fibroblasts are fully differentiated cells, providing structural support for the heart [59]. Following myocardial infarction, fibroblasts become activated, migrate to the injury site, and proliferate. The vast pool of endogenous cardiac fibroblasts in the heart could serve as a potential source of cardiomyocytes for regenerative therapy if it were possible to fully reprogram the fibroblasts into functional beating cardiomyocytes in vivo. To accomplish this, lineage-tracing experiments have been performed to track the origin of putative iCLMs. To track the fibroblast cells, a mouse Fsp1-Cre transgenic line is used, in which Cre 
recombinase is expressed under the promoter of fibroblast maker genes, FSP1. When intercrossed with an R26R-lacZ reporter line, only fibroblasts and their progeny are labeled by $\beta$-galactosidase [60]. Four transcriptional factors (Gata4, Mef2C, Tbx5, Hand2), or even three of them (Gata4, Mef2C, Tbx5), are locally delivered into dividing fibroblasts using a retrovirus expression system following myocardial infarction [59,60]. After 4 weeks, cells co-expressing $\beta$-galactosidase and $\alpha$-actinin in the infarct zone are detected. These cells have well-formed sarcomeres and express sarcomeric markers. In addition, the reprogrammed iCLMs have a normal contractile potential and are electrically coupled with endogenous cardiomyocytes and other iCLMs. Injured myocardium infected with GHMT retroviruses relieves the worsening of the heart function after 3 weeks of myocardial infarction. Ejection fraction, stroke volume, and cardiac output are significantly improved 8-12 weeks after injection [59]. Co-injecting thymosin $\beta 4$ and GMT further improves ejection fraction and cardiac output 8 weeks after infarction. These results demonstrate that GMT or GMTH can reprogram cardiac fibroblasts into iCLMs, resulting in a reduction of infarct size and improved cardiac function. The strategy bypasses many of the obstacles associated with cellular transplantation, providing a potential method for cardiac regeneration using patient-derived cells.

\subsection{Conclusion}

The past decade has improved our knowledge of chemical biology and regenerative medicine. A profound understanding of cardiomyogenesis will be required for the development of advanced therapeutics to treat ischemic heart diseases. Small molecules are not only valuable in inducing cardiomyocyte generation but also provide insight into the signaling networks and molecular mechanisms underlying these processes. Future studies will likely include identification of chemical regulators capable of reprogramming fibroblasts into cardiomyocytes. It will be essential to discover and study small molecules that can induce regeneration of preexisting cardiomyocytes. Further studies of heart development and regeneration, in combination with chemical genetics, will have great potential to advance our knowledge of and therapeutics in the treatment of cardiovascular diseases.

\section{Acknowledgements}

We acknowledge members of our laboratories for comments on the manuscript and helpful discussions. This research was supported in part by grants from National Basic Research Program of China (2013CB945300 and 2012CB944500; TPZ), Shanghai Pujiang Program (11PJ1401600), and National Science Foundation of China (Grant 31172173; TPZ), as well as the National Institutes of Health of America (NS064852; TPZ).

\section{References}

(1) Bernstein, H.S. and Srivastava, D. (2012) Stem cell therapy for cardiac disease. Pediatric Research, 71 (4 Pt 2), 491-499. 
(2) Schoenhard, J.A. and Hatzopoulos, A.K. (2010) Stem cell therapy: pieces of the puzzle. Journal of Cardiovascular Translational Research, 3 (1), 49-60.

(3) Perin, E.C., Willerson, J.T., Pepine, C.J. et al. (2012) Effect of transendocardial delivery of autologous bone marrow mononuclear cells on functional capacity, left ventricular function, and perfusion in chronic heart failure: the FOCUS-CCTRN trial. JAMA: Journal of the American Medical Association, 307 (16), 1717-1726.

(4) Wilmut, I., Schnieke, A.E., McWhir, J. et al. (1997) Viable offspring derived from fetal and adult mammalian cells. Nature, 385 (6619), 810-813.

(5) Cowan, C.A., Atienza, J., Melton, D.A., and Eggan, K. (2005) Nuclear reprogramming of somatic cells after fusion with human embryonic stem cells. Science, 309 (5739), 1369-1373.

(6) Tada, M., Takahama, Y., Abe, K. et al. (2001) Nuclear reprogramming of somatic cells by in vitro hybridization with ES cells. Current Biology, 11 (19), 1553-1558.

(7) Takahashi, K. and Yamanaka, S. (2006) Induction of pluripotent stem cells from mouse embryonic and adult fibroblast cultures by defined factors. Cell, 126 (4), 663-676.

(8) Takahashi, K., Tanabe, K., Ohnuki, M. et al. (2007) Induction of pluripotent stem cells from adult human fibroblasts by defined factors. Cell, 131 (5), 861-872.

(9) Yu, J., Vodyanik, M.A., Smuga-Otto, K. et al. (2007) Induced pluripotent stem cell lines derived from human somatic cells. Science, 318 (5858), 1917-1920.

(10) Zaret, K.S. and Carroll, J.S. (2011) Pioneer transcription factors: establishing competence for gene expression. Genes \& Development, 25 (21), 2227-2241.

(11) Nie, B., Wang, H., Laurent, T., and Ding, S. (2012) Cellular reprogramming: a small molecule perspective. Current Opinion in Cell Biology, 24 (6), 784-792.

(12) Huangfu, D., Maehr, R., Guo, W. et al. (2008) Induction of pluripotent stem cells by defined factors is greatly improved by small-molecule compounds. Nature Biotechnology, 26 (7), 795 797.

(13) Mikkelsen, T.S., Hanna, J., Zhang, X. et al. (2008) Dissecting direct reprogramming through integrative genomic analysis. Nature, 454 (7200), 49-55.

(14) Esteban, M.A., Wang, T., Qin, B. et al. (2010) Vitamin C enhances the generation of mouse and human induced pluripotent stem cells. Cell Stem Cell, 6 (1), 71-79.

(15) Mali, P., Chou, B.K., Yen, J. et al. (2010) Butyrate greatly enhances derivation of human induced pluripotent stem cells by promoting epigenetic remodeling and the expression of pluripotencyassociated genes. Stem Cells, 28 (4), 713-720.

(16) Cloos, P.A., Christensen, J., Agger, K., and Helin, K. (2008) Erasing the methyl mark: histone demethylases at the center of cellular differentiation and disease. Genes \& Development, 22 (9), $1115-1140$.

(17) Miller, S.J. (2004) Cellular and physiological effects of short-chain fatty acids. Mini Reviews in Medicinal Chemistry, 4 (8), 839-845.

(18) Lin, T., Ambasudhan, R., Yuan, X. et al. (2009) A chemical platform for improved induction of human iPSCs. Nature Methods, 6 (11), 805-808.

(19) Willis, B.C. and Borok, Z. (2007) TGF-beta-induced EMT: mechanisms and implications for fibrotic lung disease. American Journal of Physiology - Lung Cellular and Molecular Physiology, 293 (3), L525-534.

(20) Thiery, J.P. and Sleeman, J.P. (2006) Complex networks orchestrate epithelial-mesenchymal transitions. Nature Reviews Molecular Cell Biology, 7 (2), 131-142.

(21) Chou, Y.F., Chen, H.H., Eijpe, M. et al. (2008) The growth factor environment defines distinct pluripotent ground states in novel blastocyst-derived stem cells. Cell, 135 (3), 449-461.

(22) Shi, Y., Desponts, C., Do, J.T. et al. (2008) Induction of pluripotent stem cells from mouse embryonic fibroblasts by Oct4 and Klf4 with small-molecule compounds. Cell Stem Cell, 3 (5), 568-574.

(23) Zhu, S., Li, W., Zhou, H. et al. (2010) Reprogramming of human primary somatic cells by OCT4 and chemical compounds. Cell Stem Cell, 7 (6), 651-655.

(24) Hewitson, K.S. and Schofield, C.J. (2004) The HIF pathway as a therapeutic target. Drug Discovery Today, 9 (16), 704-711.

(25) Pelicano, H., Martin, D.S., Xu, R.H., and Huang, P. (2006) Glycolysis inhibition for anticancer treatment. Oncogene, 25 (34), 4633-4646. 
(26) Li, Y., Zhang, Q., Yin, X. et al. (2011) Generation of iPSCs from mouse fibroblasts with a single gene, Oct4, and small molecules. Cell Research, 21 (1), 196-204.

(27) Cole, M.F., Johnstone, S.E., Newman, J.J. et al. (2008) Tcf3 is an integral component of the core regulatory circuitry of embryonic stem cells. Genes \& Development, 22 (6), 746-755.

(28) Cao, Y. (2013) Regulation of germ layer formation by pluripotency factors during embryogenesis. Cell \& Bioscience, 3 (1), 15.

(29) Marson, A., Foreman, R., Chevalier, B. et al. (2008) Wnt signaling promotes reprogramming of somatic cells to pluripotency. Cell Stem Cell, 3 (2), 132-135.

(30) Cartwright, P., McLean, C., Sheppard, A. et al. (2005) LIF/STAT3 controls ES cell self-renewal and pluripotency by a Myc-dependent mechanism. Development (Cambridge, England), 132 (5), 885-896.

(31) Samavarchi-Tehrani, P., Golipour, A., David, L. et al. (2010) Functional genomics reveals a BMP-driven mesenchymal-to-epithelial transition in the initiation of somatic cell reprogramming. Cell Stem Cell, 7 (1), 64-77.

(32) Li, R., Liang, J., Ni, S. et al. (2010) A mesenchymal-to-epithelial transition initiates and is required for the nuclear reprogramming of mouse fibroblasts. Cell Stem Cell, 7 (1), 51-63.

(33) Das, S., Becker, B.N., Hoffmann, F.M., and Mertz, J.E. (2009) Complete reversal of epithelial to mesenchymal transition requires inhibition of both ZEB expression and the Rho pathway. BMC Cell Biology [Electronic Resource], 10, 94.

(34) Polo, J.M. and Hochedlinger, K. (2010) When fibroblasts MET iPSCs. Cell Stem Cell, 7 (1), $5-6$.

(35) Ichida, J.K., Blanchard, J., Lam, K. et al. (2009) A small-molecule inhibitor of tgf-Beta signaling replaces sox 2 in reprogramming by inducing nanog. Cell Stem Cell, 5 (5), 491-503.

(36) Efe, J.A., Hilcove, S., Kim, J. et al. (2011) Conversion of mouse fibroblasts into cardiomyocytes using a direct reprogramming strategy. Nature Cell Biology, 13 (3), 215-222.

(37) Poss, K.D., Wilson, L.G., and Keating, M.T. (2002) Heart regeneration in zebrafish. Science, 298 (5601), 2188-2190.

(38) Wang, J., Panakova, D., Kikuchi, K. et al. (2011) The regenerative capacity of zebrafish reverses cardiac failure caused by genetic cardiomyocyte depletion. Development (Cambridge, England), 138 (16), 3421-3430.

(39) Gonzalez-Rosa, J.M., Martin, V., Peralta, M. et al. (2011) Extensive scar formation and regression during heart regeneration after cryoinjury in zebrafish. Development (Cambridge, England), 138 (9), 1663-1674.

(40) Parente, V., Balasso, S., Pompilio, G. et al. (2013) Hypoxia/reoxygenation cardiac injury and regeneration in zebrafish adult heart. PLoS One, 8 (1), e53748.

(41) Kikuchi, K., Gupta, V., Wang, J. et al. (2011) tcf21 + epicardial cells adopt non-myocardial fates during zebrafish heart development and regeneration. Development (Cambridge, England), 138 (14), 2895-2902.

(42) Lepilina, A., Coon, A.N., Kikuchi, K. et al. (2006) A dynamic epicardial injury response supports progenitor cell activity during zebrafish heart regeneration. Cell, 127 (3), 607-619.

(43) Kim, J., Wu, Q., Zhang, Y. et al. (2010) PDGF signaling is required for epicardial function and blood vessel formation in regenerating zebrafish hearts. Proceedings of the National Academy of Sciences of the United States of America, 107 (40), 17206-17210.

(44) Kikuchi, K., Holdway, J.E., Major, R.J. et al. (2011) Retinoic acid production by endocardium and epicardium is an injury response essential for zebrafish heart regeneration. Developmental Cell, 20 (3), 397-404.

(45) Porrello, E.R., Mahmoud, A.I., Simpson, E. et al. (2011) Transient regenerative potential of the neonatal mouse heart. Science, 331 (6020), 1078-1080.

(46) Haubner, B.J., Adamowicz-Brice, M., Khadayate, S. et al. (2012) Complete cardiac regeneration in a mouse model of myocardial infarction. Aging (Albany NY), 4 (12), 966-977.

(47) Bergmann, O., Bhardwaj, R.D., Bernard, S. et al. (2009) Evidence for cardiomyocyte renewal in humans. Science, 324 (5923), 98-102.

(48) Kajstura, J., Gurusamy, N., Ogorek, B. et al. (2010) Myocyte turnover in the aging human heart. Circulation Research, 107 (11), 1374-1386. 
(49) Jopling, C., Sleep, E., Raya, M. et al. (2010) Zebrafish heart regeneration occurs by cardiomyocyte dedifferentiation and proliferation. Nature, 464 (7288), 606-609.

(50) Kikuchi, K., Holdway, J.E., Werdich, A.A. et al. (2010) Primary contribution to zebrafish heart regeneration by gata4 ( + ) cardiomyocytes. Nature, 464 (7288), 601-605.

(51) Senyo, S.E., Steinhauser, M.L., Pizzimenti, C.L. et al. (2013) Mammalian heart renewal by pre-existing cardiomyocytes. Nature, 493 (7432), 433-436.

(52) Loffredo, F.S., Steinhauser, M.L., Gannon, J., and Lee, R.T. (2011) Bone marrow-derived cell therapy stimulates endogenous cardiomyocyte progenitors and promotes cardiac repair. Cell Stem Cell, 8 (4), 389-398.

(53) Smart, N., Bollini, S., Dube, K.N. et al. (2011) De novo cardiomyocytes from within the activated adult heart after injury. Nature, 474 (7353), 640-644.

(54) Choi, W.Y., Gemberling, M., Wang, J. et al. (2013) In vivo monitoring of cardiomyocyte proliferation to identify chemical modifiers of heart regeneration. Development (Cambridge, England), 140 (3), 660-666.

(55) Ni, T.T., Rellinger, E.J., Mukherjee, A. et al. (2011) Discovering small molecules that promote cardiomyocyte generation by modulating Wnt signaling. Chemistry \& Biology, 18 (12), 16581668.

(56) Willems, E., Spiering, S., Davidovics, H. et al. (2011) Small-molecule inhibitors of the Wnt pathway potently promote cardiomyocytes from human embryonic stem cell-derived mesoderm. Circulation Research, 109 (4), 360-364.

(57) Ieda, M., Fu, J.D., Delgado-Olguin, P. et al. (2010) Direct reprogramming of fibroblasts into functional cardiomyocytes by defined factors. Cell, 142 (3), 375-386.

(58) Ieda, M., Tsuchihashi, T., Ivey, K.N. et al. (2009) Cardiac fibroblasts regulate myocardial proliferation through beta1 integrin signaling. Developmental Cell, 16 (2), 233-244.

(59) Song, K., Nam, Y.J., Luo, X. et al. (2012) Heart repair by reprogramming non-myocytes with cardiac transcription factors. Nature, 485 (7400), 599-604.

(60) Qian, L., Huang, Y., Spencer, C.I. et al. (2012) In vivo reprogramming of murine cardiac fibroblasts into induced cardiomyocytes. Nature, 485 (7400), 593-598. 


\title{
4 \\ Challenges and New Directions for Cardiac Reprogramming
}

\author{
Young-Jae Nam ${ }^{1}$ and Nikhil Munshi ${ }^{2}$ \\ ${ }^{1}$ Department of Medicine, Division of Cardiovascular Medicine, Vanderbilt University, USA \\ ${ }^{2}$ Internal Medicine, University of Texas Southwestern Medical Center, USA
}

\subsection{Introduction}

Despite remarkable advances in cardiovascular medicine, ischemic heart disease, including myocardial infarction and subsequent heart failure, is the number one cause of morbidity and mortality worldwide [1]. The fundamental yet unresolved problem is that the adult heart irreversibly loses millions to billions of cardiomyocytes during myocardial infarction due to an inability to regenerate cardiomyocytes [2]. Injured and aging cardiomyocytes are replaced by fibrotic scar. This leads to loss of pump function and provides a substrate for life-threatening arrhythmias, a common mode of death in heart failure. Thus, the ability to generate new cardiomyocytes remains an imperative scientific focus.

\subsection{Strategies for Heart Repair}

One approach under active investigation is to transplant stem/progenitor cells, which have the potential to differentiate into cardiomyocytes, to repair the injured heart. One obvious potential source for this approach is human embryonic stem cells (ESCs), which can efficiently differentiate into cardiomyocytes and have the potential to regenerate the heart

Chemical Biology in Regenerative Medicine: Bridging Stem Cells and Future Therapies, First Edition. Edited by Charles C. Hong, Ada S. Ao, and Jijun Hao.

(C) 2014 John Wiley \& Sons, Ltd. Published 2014 by John Wiley \& Sons, Ltd. 
[3]. Besides ethical issues, however, two major problems limit this strategy from clinical application: immunological rejection (because ESCs are foreign to the body) and teratoma formation from undifferentiated cells. An alternative approach is to identify stem/progenitor cells that can differentiate into cardiomyocytes as the source for cell therapy. Although this approach has been extensively tested in animal models and numerous clinical trials over the past decade, clinical efficacy has been modest at best and is not supported by clear mechanistic insights [4]. Furthermore, transdifferentiation of transplanted stem/progenitor cells into cardiomyocytes has not been clearly and consistently demonstrated. Rather, it has been a general consensus that the beneficial effect of cell-based therapy results from paracrine effects of transplanted cells [5]. Induced pluripotent stem cells (iPSCs) or iPSC-derived cardiomyocytes are another attractive cell source for cell-replacement therapy and heart repair [6]. There is no doubt that iPSC reprogramming, followed by directed differentiation, offers a powerful approach to regenerating any cell type and permits in vitro production of personalized cell therapies. Before this strategy can be translated into therapeutic application, however, tumor formation from undifferentiated cells and unexpected immunogenicity must be resolved [7]. Poor survival rate of transplanted cells is another obstacle preventing clinical use of cell replacement-based strategies. As few as $\sim 15 \%$ of transplanted cells remain in the heart without leaking from the injection site or entering systemic circulation [8], with only $10 \%$ of the $15 \%$ surviving more than a week in the heart [9]. Overall, cell transplantation-based therapy for cardiac repair will remain inefficient unless the delivery systems and survival of transplanted cells improve. Therefore, there is a major need to develop an entirely new therapeutic strategy that will eliminate the obstacles facing current cell-based therapies for post-heart attack intervention.

More than half of the cells in the heart are fibroblasts. They are activated during injury and lead to cardiac fibrosis and scar formation, thereby impeding contractility, and contribute to adverse remodeling and conduction abnormalities. Therefore, directly targeting cardiac fibroblasts in the heart so that they transform into new cardiac muscle tissue is a particularly attractive strategy for heart repair after injury (Figure 4.1). In fact, this direct phenotypic conversion from one fully differentiated cell type into another has long been described in studies, but it was previously restricted to related cell types for which a single master transcription factor controls cell fate. Examples include (i) fibroblasts to myofibers, (ii) fibroblasts to smooth muscle cells, (iii) B lymphocytes to macrophages, and (iv) inner-ear support cells to hair cells [10-13]. The discovery that a combination of transcription factors was required to reprogram iPSCs, rather than a single master transcription factor, opened up new avenues for specific lineage conversions. As a result, lineage reprogramming has been extended to other medically useful cell types that require more complex transcriptional regulation to establish their identities, including pancreatic $\beta$-cells, neurons, hepatocytes, and cardiomyocytes [14-18].

\subsection{Direct Reprogramming Approaches}

A heart-repair strategy using direct reprogramming has multiple advantages over current stem cell-based approaches. First, there is no need for ex vivo manipulation of cells and reintroduction into the heart, since reprogramming occurs in vivo [19,20]. This eliminates the obstacles associated with efficient transplantation and integration of engrafted cells 

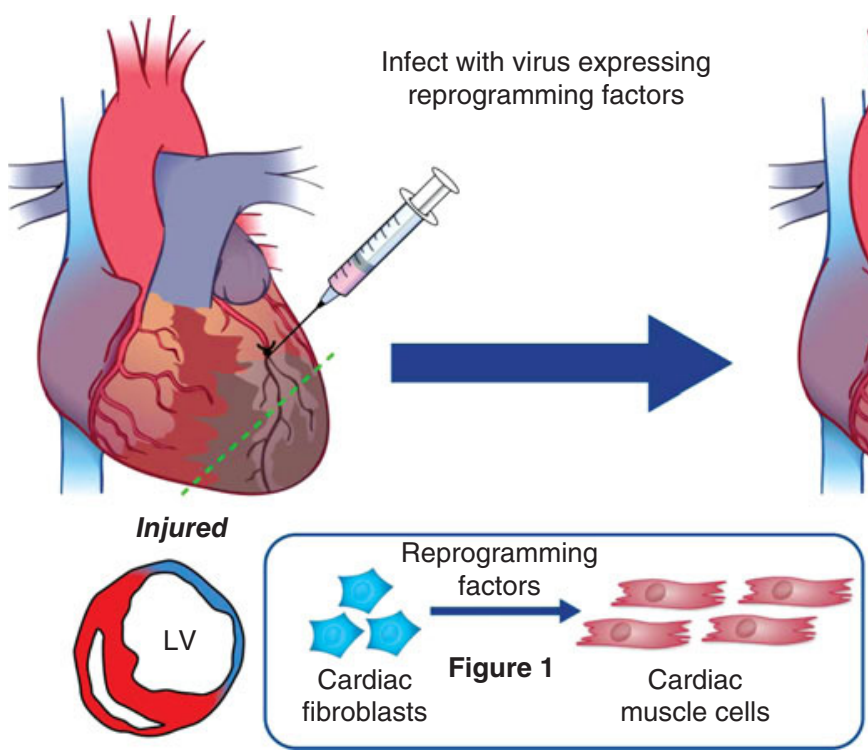

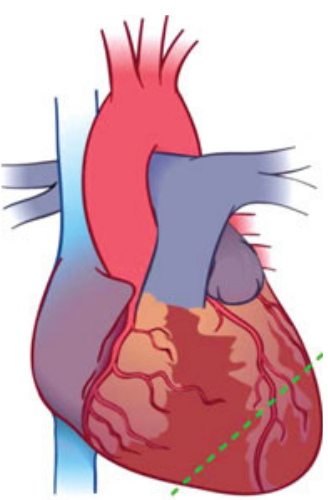

Repaired

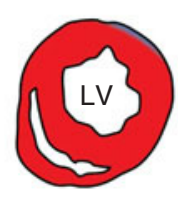

Figure 4.1 Heart repair by reprogramming of non-myocytes into cardiomyocyte-like cells in vivo. Immediately after left anterior descending (LAD) coronary artery ligation to induce myocardial infarction, a viral cocktail of reprogramming factors is directly injected into the border zone adjacent to the infarcted myocardium. The forced expression of reprogramming factors in non-myocytes in the heart after myocardial infarction induces new cardiomyocytelike cells and leads to the improvement of contractile function and reduction of scar formation. $L V$, left ventricle. (Adapted by permission from Macmillan Publishers Ltd: Nature Medicine [20], copyright (2013))

into the functioning myocardium. Second, this lineage reprogramming approach does not transverse a stem cell-like state, which reduces the likelihood of teratoma formation from undifferentiated cells. Third, this strategy is expected to reduce cardiac fibrosis by directly targeting cardiac fibroblasts, the principal mediators of cardiac fibrosis and scar formation after myocardial infarction (MI [21]. Fibrotic scar formation at the site of an infarct and interstitial fibrosis of adjacent myocardium act as additional barriers to cardiac repair and contribute to loss of pump function, pathological remodeling, and susceptibility to arrhythmias [22].

Fibroblasts can be induced to become skeletal and smooth muscle cells by ectopic expression of a single transcription factor, such as MyoD and Myocardin, respectively; however, a master transcription factor has not been identified for cardiac muscle despite exhaustive searches over the past 2 decades $[10,11]$. It is clear that cardiac cell fate is determined by a complex network of core cardiac transcription factors rather than a single transcription factor [23]. Based on this knowledge, Srivastava's group made an important first step toward direct cardiac reprogramming. Ieda et al. first reported the ability of exogenous transcription factors to direct mouse fibroblasts toward a cardiac fate [18]. First, they identified 14 candidate transcription factors that are important in heart development. Then they created a cardiac-specific $\alpha \mathrm{MHC}-\mathrm{GFP}$ transgenic mouse to isolate neonatal cardiac fibroblasts. Using these reagents, they identified three transcription factors - Gata4, Mef2c, and Tbx5 
(also known as GMT) - that were sufficient to induce cardiac phenotype in mouse fibroblasts. However, the reprogramming efficiency remains low; 5-15\% of fibroblasts express cardiac markers, and only $0.5 \%$ of cardiac marker-expressing cells contract. The Olson group advanced this reprogramming protocol, showing that inclusion of another cardiac transcription factor, Hand $2(\mathrm{H})$, in the GMT cocktail significantly increases the reprogramming efficiency. Song et al. demonstrated in side-by-side comparisons that GHMT is around 4-fold more effective than GMT in cardiac reprogramming in vitro. Importantly, they focused on adult fibroblasts (cardiac and tail-tip), which are less susceptible to reprogramming than immature cell types and more likely to represent the substrate for in vivo therapeutic reprogramming [20]. The use of adult fibroblasts for cardiac reprogramming also diminishes the likelihood that residual embryonic cardiac progenitors present in neonatal preparations might contaminate fibroblast preparations. The enhanced reprogramming power of GHMT is further demonstrated by its ability to induce a contractile phenotype in adult tail-tip fibroblasts that is not achieved by GMT. A muscle-specific microRNA, miR-1, alone or in combination with other muscle-specific microRNAs (miR-133, -208, and -499), has also been reported to activate cardiac gene expression in fibroblasts with low efficiency [24]. Although a microRNA-based reprogramming approach can induce global transcriptional changes favoring a cardiac phenotype, sarcomere-like structures, calcium transients, and spontaneous contraction of reprogrammed cells have not been reported. Other groups showed that different combinations of transcription factors (GMT Myocardin and GHMT Nkx2-5) are able to induce cardiac phenotype in mouse fibroblasts $[25,26]$. These results indicate that multiple combinations of cardiac regulators can initiate the cardiac differentiation program because they function within complex regulatory networks that involve feed-forward and autoregulatory interactions.

The most important turning point for cardiac reprogramming as a potential cardiac regenerative therapy was the unexpected success of in vivo reprogramming [19,20, 24, 27]. Srivastava and Olson's groups independently showed that in vivo reprogramming is feasible using the same reprogramming factor combinations identified in in vitro reprogramming (GMT and GHMT, respectively) [19,20]. Both groups used retroviruses to induce reprogramming of activated cardiac fibroblasts in the infarct zone of mouse infarct models, because retroviruses infect only proliferating fibroblasts rather than quiescent cardiomyocytes. To identify the origin of newly generated cardiomyocytes, both groups performed lineage-tracing experiments using FSP1 Cre or Periostin Cre/Rosa26LacZ mice, in which they could label fibroblasts with $\beta$-galactosidase following injury. There has been concern that cardiac injury or viral transduction might unintentionally activate the promoter of these reporters. To exclude this possibility, the Olson group generated a new inducible lineage marker for noncardiomyocytes, in which MerCreMer was knocked into the Tcf 21 locus by homologous recombination. Tcf21 is specific for non-myocytes within the heart, with no expression in cardiomyocytes. Using this new inducible non-myocyte lineage marker, they substantiated their conclusion that newly generated cardiomyocytes originate from nonmyocytes. However, it is still unclear whether the new myocytes were derived only from cardiac fibroblasts, rather than from other non-myocyte cell types, because a true fibroblastspecific lineage marker is unavailable. Thus, these studies cannot exclude the contribution of the other reprogrammed cell types to cardiac repair by GMT or GHMT. Interestingly, the reprogramming efficiency in vivo seems to be higher than that in vitro. This result suggests that the in vivo environment, which contains cardiomyocytes and other cell types, as well 
as extracellular factors, is more permissive for reprogramming than plastic tissue culture dishes. In addition, constant electrophysiological activation and mechanical contraction in the intact heart likely have salutary effects on cardiac reprogramming. More importantly, both studies demonstrated that introduction of reprogramming factors (GMT or GHMT) improved contractile function and reduced scar formation after myocardial infarction in a mouse model. This functional benefit seems to be greater than predicted based on the number of newly generated cardiomyocytes observed in lineage-tracing experiments. This may indicate that introduction of cardiac reprogramming factors upon injury enhances contractile function through mechanisms beyond simple directing of fibroblasts toward a cardiomyocyte cell fate. Potential mechanisms include (i) promoting neoangiogenesis, (ii) preventing cardiomyocyte death, (iii) inhibiting fibroblast activation/proliferation, and (iv) facilitating the differentiation of cardiac progenitors to cardiomyocytes following injury. Further studies to clarify these additional mechanisms are warranted prior to potential clinical application.

One common hurdle for the reprogramming approach is induction of desired cell types in human fibroblasts. As every potential clinical application of reprogramming can only be realized in human cells, reprogramming of human fibroblasts is a mandatory step to practical usage. However, human-cell reprogramming is known to be less efficient and requires longer time in culture, as indicated by human iPSC and neuron reprogramming studies [28-30]. Nam et al. first reported successful induction of cardiac phenotype in human fibroblasts with the addition of myocardin to GHMT [31]. Although this new combination of factors is able to induce multiple cardiac markers, evidence suggests that cardiac reprogramming is incomplete. For example, the global transcriptional changes in reprogrammed cells are similar but not identical to human cells, and sarcomere-like structures, calcium transients, and spontaneous contraction only occur in a very small subset of reprogrammed cells after a prolonged culture period. These results indicate that higher barriers to cardiac reprogramming are likely to exist in human fibroblasts. The protocol was modified to include two muscle-specific microRNAs, miR-1 and miR-133, making Mef2c dispensable in the reprogramming cocktail. The rationale for using these musclespecific microRNAs is based on previous findings that miR-1 and miR-133 are regulated by Mef2c [32]. Subsequent studies by two other groups also showed reprogramming of human fibroblasts toward a cardiac cell fate using a slightly different combination of transcription factors, namely GMT-Myocardin-Mesp1 with or without the nuclear hormone receptor ESRRG [33,34]. In general, conversion of human fibroblasts toward a cardiac cell fate relies on more reprogramming factors, requires a longer period of time, and is less efficient than mouse cardiac reprogramming. The different requirements for reprogramming of mouse and human fibroblasts likely reflect differences in the mouse and human fibroblast populations and the susceptibility of cardiac genes to activation in these different cells.

\subsection{Current Challenges}

The examples provided of cardiac reprogramming suggest a potentially promising and entirely new heart-repair strategy. However, like any other innovative therapeutic intervention, this new approach faces numerous biological and technical obstacles. We will discuss a number of current challenges here. 
The most rigorous criterion for confirming cardiac phenotype in reprogrammed cells is contractility. Based on this criterion, reprogramming efficiency remains low despite the development of innovative methods. Efficiency is especially important in direct reprogramming because the resulting cardiomyocyte-like cell does not proliferate and cannot be maintained indefinitely in culture. Given the fact that billions of cardiomyocytes are lost during an infarction, optimal cardiac reprogramming efficiency will be required to contribute to meaningful clinical application. Furthermore, the current cardiac-reprogramming protocol generates a wide spectrum of heterogeneous phenotypes that can be classified in multiple groups: (i) single cardiac marker-positive cells without sarcomeric structure, (ii) multiple cardiac marker-positive cells without sarcomeric structure, (iii) multiple cardiac marker-positive cells with sarcomeric structure, (iv) contractile cells with action potential upon electrical stimulation, and (v) contractile cells with spontaneous action potentials. Reprogrammed cells that develop an organized sarcomeric structure and contractility represent all three subtypes of cardiomyocyte, including atrial, ventricular, and pacemaker cells. This raises the concern that heterogeneity in cell-type identity and maturity may increase the risk of conduction abnormalities in vivo as the infarct zone is already at a higher risk for arrhythmogenesis. Thus, future efforts will need to focus on improving the efficacy of cardiac reprogramming in terms of cell-type specificity and the maturity of individual cardiomyocytes. Why is cardiac reprogramming a relatively inefficient and heterogeneous process? This may reflect a specific stoichiometry of each reprogramming factor in activating the cardiac development program, which is achieved in a very small subset of the starting cell population. Because at least three transcription factors are required for cardiac reprogramming, this lower efficiency is not unexpected when compared to other reprogramming processes dominated by a single master regulator. Another possible explanation is that heterogeneity in the starting population of fibroblasts may contribute to reprogramming inefficiency because only a subset of cells is susceptible to reprogramming. An additional factor by which to explain cardiac reprogramming inefficiency and heterogeneity is that GHMT factors play multiple roles during embryonic development and perhaps in lineage conversions. Thus, the simultaneous action of all four factors may promote multiple alternative cell fates that detract from the goal of creating cardiomyocytes. Also, the role of reprogramming repressors should be considered; their deletion may markedly enhance reprogramming efficiency. In this regard, a recent study by Hanna's group demonstrated that deletion of a single repressor, Mbd3, is able to produce iPSCs with near $100 \%$ efficiency when used in combination with Yamanaka factors [35]. The search for similar roadblocks in cardiac reprogramming will be an important research focus in this field.

Induced cardiomyocytes are relatively immature, and only a small fraction displays the strong contractility, well-developed sarcomeres, and binucleation associated with adult cardiomyocytes. In addition, none of the induced cardiomyocytes from any published studies displays rod-shaped adult cardiomyocyte morphology. This is unsurprising when we consider how current reprogramming protocols were developed. The selection of cardiac reprogramming factors has been based solely on embryonic heart development $[18,20]$. Additional factors that can induce the adult cardiac phenotype will be required, and further study on transcriptional regulation of postnatal cardiac muscle maturation is warranted to achieve this goal.

The current in vivo reprogramming strategy for myocardial infarction in a mouse model is to directly inject a viral cocktail of reprogramming factors into the infarcted myocardium 
during open-chest surgery [19,20, 24, 27]. Given the potential for teratogenic viral insertions in the genome and the potential inflammatory complications associated with viral delivery, it will be important to develop nonintegrative and nonviral methods for safe clinical application. Small molecules can be a powerful tool for nonviral reprogramming. They have enhanced transcripton factor-mediated iPSC and neuronal reprogramming [36-43]. Furthermore, iPSCs have recently been generated using a seven-molecule cocktail, without any viral transduction, suggesting that a new chemical strategy for reprogramming is possible [44]. A couple of small molecules - JAK inhibitor and transforming growth factor beta (TGF) $\beta$ - demonstrated synergistic effects with transcription factors used in cardiac reprogramming [24, 34, 45]. Although these small molecules are unable to replace transcription factors or microRNAs in achieving cardiac reprogramming, these studies provide a starting point from which to develop a virus-free cardiacreprogramming protocol in the future. MicroRNAs are another alternative to transcription factors in cardiac reprogramming. As we discussed earlier, miR-1 alone was sufficient to induce cardiac phenotype in mouse fibroblasts [24], and a combination of miR-1 and miR-133 is able to replace the transcription factor Mef2c in human cardiac reprogramming [31]. MicroRNA-based cardiac reprogramming is particularly attractive for heart repair because the expression of microRNAs is efficiently manipulated in muscle tissue in vivo using miR-mimics or anti-miRs. Reprogramming fibroblasts to cardiomyocytes using purely chemical means will be a critical step toward therapeutic application of this strategy in the future. Replacing cardiogenic transcription factors with small molecules or synthetic oligonucleotides with cardiogenic activity will have long-term therapeutic possibilities.

From a clinical standpoint, direct injection of reprogramming factors into the myocardium after a heart attack is far from realistic, and the risks associated with this procedure outweigh the potential benefits. It is also important to develop less invasive procedures for the introduction of reprogramming factors, perhaps a catheter-based delivery during percutaneous coronary intervention (PCI) after a myocardial infarction with a virus-free reprogramming protocol. Clinical translation of direct reprogramming will benefit from safe and effective delivery methods previously developed for cell transplantation. As a starting point, reprogramming factors can be directly delivered during a coronary artery bypass graft surgery (CABG) after a heart attack.

The heart is not just a muscle pump solely composed of force-generating muscle cells, but a much more complex organ system made up of distinctive cell types that are highly orchestrated for effective blood pumping. Myocytes in different regions of the heart also display distinct phenotypes. Therefore, full restoration of cardiac function after injury will require regeneration of the cell types that are substantially compromised upon cardiac injury. In this regard, smooth muscle cells, endothelial cells, and angioblast-like progenitor cells have been successfully generated using direct reprogramming strategies [11, 46, 47], and inclusion of a vascular-endothelial growth factor (VEGF)-expressing virus with GMT enhances functional recovery of the injured myocardium, possibly through neovascularization [48]. In addition, forced expression of Tbx18 or activated Notch in ventricular cardiomyocytes or Tbx3 in atrial cells is sufficient to generate conduction system cells [49-51]. However, direct reprogramming of fibroblasts toward specific cardiomyocyte subtypes, including atrial, ventricular, and pacemaker myocytes, remains to be investigated. In this regard, multiple subtypes of neurons, such as excitatory, dopaminergic, and motor, have been directly 
reprogrammed from fibroblasts using distinctive combinations of transcription factors and may inform studies in cardiomyocyte subtypes.

\subsection{Conclusion}

Over the past few years cardiac reprogramming has drawn enormous scientific attention, mainly due to an unmet need for new heart-repair strategies. As we have discussed here, there are numerous challenges in cardiac reprogramming that must be met before any potential clinical application. We have learned from numerous cell-transplantation studies in the past that simply introducing new cells into the heart has minimal benefits. Practical use of cardiac reprogramming will require efforts to address the main challenges facing this field: (i) inefficiency, (ii) heterogeneity, (iii) development of a virus-free cardiac-reprogramming protocol, (iv) provision of a safe and effective delivery method, (v) elucidation of the mechanical basis of cardiac reprogramming, and (vi) demonstration of the efficacy and safety of the procedure in a large-animal model prior to human clinical trial.

\section{Acknowledgements}

We are grateful to Jose Cabrera for excellent graphical assistance and to many members of the Olson lab for their intellectual input. YJN is supported by a K08 Award from the NHLBI and work in the lab of NVM is funded by a K08 Award from the NHLBI, a Career Award for Medical Scientists from the Burroughs Wellcome Fund, a Basil O'Connor Starter Scholar Award from the March of Dimes Foundation, and a Disease Oriented Clinical Scholar Award from UT Southwestern Medical Center.

\section{References}

(1) Go, A.S., Mozaffarian, D., Roger, V.L. et al. (2013) Heart disease and stroke statistics-2013 update: a report from the American heart association. Circulation, 127 (1), e6-e245.

(2) Murry, C.E., Reinecke, H., and Pabon, L.M. (2006) Regeneration gaps: observations on stem cells and cardiac repair. Journal of the American College of Cardiology, 47 (9), 1777-1785.

(3) Garry, D.J. and Olson, E.N. (2006) A common progenitor at the heart of development. Cell, 127 (6), 1101-1104.

(4) Segers, V.F. and Lee, R.T. (2008) Stem-cell therapy for cardiac disease. Nature, 451 (7181), 937-942.

(5) Garbern, J.C. and Lee, R.T. (2013) Cardiac stem cell therapy and the promise of heart regeneration. Cell Stem Cell, 12 (6), 689-698.

(6) Takahashi, K. and Yamanaka, S. (2006) Induction of pluripotent stem cells from mouse embryonic and adult fibroblast cultures by defined factors. Cell, 126 (4), 663-676.

(7) Zhao, T., Zhang, Z.N., Rong, Z., and Xu, Y. (2011) Immunogenicity of induced pluripotent stem cells. Nature, 474 (7350), 212-215.

(8) Muller-Ehmsen, J., Whittaker, P., Kloner, R.A. et al. (2002) Survival and development of neonatal rat cardiomyocytes transplanted into adult myocardium. Journal of Molecular and Cellular Cardiology, 34 (2), 107-116.

(9) Laflamme, M.A. and Murry, C.E. (2005) Regenerating the heart. Nature Biotechnology, 23 (7), 845-856. 
(10) Davis, R.L., Weintraub, H., and Lassar, A.B. (1987) Expression of a single transfected cDNA converts fibroblasts to myoblasts. Cell, 51 (6), 987-1000.

(11) Wang, Z., Wang, D.Z., Pipes, G.C., and Olson, E.N. (2003) Myocardin is a master regulator of smooth muscle gene expression. Proceedings of the National Academy of Sciences of the United States of America, 100 (12), 7129-7134.

(12) Xie, H., Ye, M., Feng, R., and Graf, T. (2004) Stepwise reprogramming of B cells into macrophages. Cell, 117 (5), 663-676.

(13) Zheng, J.L. and Gao, W.Q. (2000) Overexpression of Math1 induces robust production of extra hair cells in postnatal rat inner ears. Nature Neuroscience, 3 (6), 580-586.

(14) Zhou, Q., Brown, J., Kanarek, A. et al. (2008) In vivo reprogramming of adult pancreatic exocrine cells to beta-cells. Nature, 455 (7213), 627-632.

(15) Vierbuchen, T., Ostermeier, A., Pang, Z.P. et al. (2010) Direct conversion of fibroblasts to functional neurons by defined factors. Nature, 463 (7284), 1035-1041.

(16) Sekiya, S. and Suzuki, A. (2011) Direct conversion of mouse fibroblasts to hepatocyte-like cells by defined factors. Nature, 475 (7356), 390-393.

(17) Huang, P., He, Z., Ji, S. et al. (2011) Induction of functional hepatocyte-like cells from mouse fibroblasts by defined factors. Nature, 475 (7356), 386-389.

(18) Ieda, M., Fu, J.D., Delgado-Olguin, P. et al. (2010) Direct reprogramming of fibroblasts into functional cardiomyocytes by defined factors. Cell, 142 (3), 375-386. PubMed PMID: 20691899.

(19) Qian, L., Huang, Y., Spencer, C.I. et al. (2012) In vivo reprogramming of murine cardiac fibroblasts into induced cardiomyocytes. Nature, 485 (7400), 593-598.

(20) Song, K., Nam, Y.J., Luo, X. et al. (2012) Heart repair by reprogramming non-myocytes with cardiac transcription factors. Nature, 485 (7400), 599-604.

(21) Zeisberg, E.M. and Kalluri, R. (2010) Origins of cardiac fibroblasts. Circulation Research, 107 (11), 1304-1312. PubMed PMID: 21106947.

(22) Brown, R.D., Ambler, S.K., Mitchell, M.D., and Long, C.S. (2005) The cardiac fibroblast: therapeutic target in myocardial remodeling and failure. Annual Review of Pharmacology and Toxicology, 45, 657-687.

(23) Olson, E.N. (2006) Gene regulatory networks in the evolution and development of the heart. Science, 313 (5795), 1922-1927.

(24) Jayawardena, T.M., Egemnazarov, B., Finch, E.A. et al. (2012) MicroRNA-mediated in vitro and in vivo direct reprogramming of cardiac fibroblasts to cardiomyocytes. Circulation Research, 110 (11), 1465-1473.

(25) Addis, R.C., Ifkovits, J.L., Pinto, F. et al. (2013) Optimization of direct fibroblast reprogramming to cardiomyocytes using calcium activity as a functional measure of success. Journal of Molecular and Cellular Cardiology, 60, 97-106.

(26) Protze, S., Khattak, S., Poulet, C. et al. (2012) A new approach to transcription factor screening for reprogramming of fibroblasts to cardiomyocyte-like cells. Journal of Molecular and Cellular Cardiology, 53 (3), 323-332.

(27) Inagawa, K., Miyamoto, K., Yamakawa, H. et al. (2012) Induction of cardiomyocyte-like cells in infarct hearts by gene transfer of Gata4, Mef2c, and Tbx5. Circulation Research, 111 (9), $1147-1156$.

(28) Takahashi, K., Tanabe, K., Ohnuki, M. et al. (2007) Induction of pluripotent stem cells from adult human fibroblasts by defined factors. Cell, 131 (5), 861-872.

(29) Zhao, Y., Yin, X., Qin, H. et al. (2008) Two supporting factors greatly improve the efficiency of human iPSC generation. Cell Stem Cell, 3 (5), 475-479.

(30) Pang, Z.P., Yang, N., Vierbuchen, T. et al. (2011) Induction of human neuronal cells by defined transcription factors. Nature, 476 (7359), 220-223.

(31) Nam, Y.J., Song, K., Luo, X. et al. (2013) Reprogramming of human fibroblasts toward a cardiac fate. Proceedings of the National Academy of Sciences of the United States of America, 110 (14), 5588-5593.

(32) Liu, N., Bezprozvannaya, S., Williams, A.H. et al. (2008) microRNA-133a regulates cardiomyocyte proliferation and suppresses smooth muscle gene expression in the heart. Genes \& Development, 22 (23), 3242-3254. 
(33) Wada, R., Muraoka, N., Inagawa, K. et al. (2013) Induction of human cardiomyocyte-like cells from fibroblasts by defined factors. Proceedings of the National Academy of Sciences of the United States of America, 110 (31), 12667-12672.

(34) Fu, J.D., Stone, N.R., Liu, L. et al. (2013) Direct reprogramming of human fibroblasts toward a cardiomyocyte-like state. Stem Cell Reports, 1 (3), 235-247

(35) Rais, Y., Zviran, A., Geula, S. et al. (2013) Deterministic direct reprogramming of somatic cells to pluripotency. Nature, 502 (7469), 65.

(36) Huangfu, D., Maehr, R., Guo, W. et al. (2008) Induction of pluripotent stem cells by defined factors is greatly improved by small-molecule compounds. Nature Biotechnology, 26 (7), 795 797.

(37) Shi, Y., Desponts, C., Do, J.T. et al. (2008) Induction of pluripotent stem cells from mouse embryonic fibroblasts by Oct4 and Klf4 with small-molecule compounds. Cell Stem Cell, 3 (5), 568-574.

(38) Mikkelsen, T.S., Hanna, J., Zhang, X. et al. (2008) Dissecting direct reprogramming through integrative genomic analysis. Nature, 454 (7200), 49-55.

(39) Firestone, A.J. and Chen, J.K. (2010) Controlling destiny through chemistry: small-molecule regulators of cell fate. ACS Chemical Biology, 5 (1), 15-34. PubMed PMID: 20000447.

(40) Tursun, B., Patel, T., Kratsios, P., and Hobert, O. (2011) Direct conversion of C. elegans germ cells into specific neuron types. Science, 331 (6015), 304-308.

(41) Kubicek, S., O’Sullivan, R.J., August, E.M. et al. (2007) Reversal of H3K9me2 by a smallmolecule inhibitor for the G9a histone methyltransferase. Molecular Cell, 25 (3), 473-481.

(42) Zhu, S., Li, W., Zhou, H. et al. (2010) Reprogramming of human primary somatic cells by OCT4 and chemical compounds. Cell Stem Cell, 7 (6), 651-655.

(43) Huangfu, D., Osafune, K., Maehr, R. et al. (2008) Induction of pluripotent stem cells from primary human fibroblasts with only Oct4 and Sox2. Nature Biotechnology, 26 (11), 12691275.

(44) Hou, P.P., Li, Y.Q., Zhang, X. et al. (2013) Pluripotent stem cells induced from mouse somatic cells by small-molecule compounds. Science, 341 (6146), 651-654.

(45) Efe, J.A., Hilcove, S., Kim, J. et al. (2011) Conversion of mouse fibroblasts into cardiomyocytes using a direct reprogramming strategy. Nature Cell Biology, 13 (3), 215-U61.

(46) Margariti, A., Winkler, B., Karamariti, E. et al. (2012) Direct reprogramming of fibroblasts into endothelial cells capable of angiogenesis and reendothelialization in tissue-engineered vessels. Proceedings of the National Academy of Sciences of the United States of America, 109 (34), 13793-13798.

(47) Kurian, L., Sancho-Martinez, I., Nivet, E. et al. (2013) Conversion of human fibroblasts to angioblast-like progenitor cells. Nature Methods, 10 (1), 77-83.

(48) Mathison, M., Gersch, R.P., Nasser, A. et al. (2012) In vivo cardiac cellular reprogramming efficacy is enhanced by angiogenic preconditioning of the infarcted myocardium with vascular endothelial growth factor. Journal of the American Heart Association, 1 (6), e005652.

(49) Kapoor, N., Liang, W., Marban, E., and Cho, H.C. (2012) Direct conversion of quiescent cardiomyocytes to pacemaker cells by expression of Tbx18. Nature Biotechnology, 31 (1), $54-62$.

(50) Rentschler, S., Yen, A.H., Lu, J. et al. (2012) Myocardial Notch signaling reprograms cardiomyocytes to a conduction-like phenotype. Circulation, 126 (9), 1058-1066.

(51) Bakker, M.L., Boink, G.J., Boukens, B.J. et al. (2012) T-box transcription factor TBX3 reprogrammes mature cardiac myocytes into pacemaker-like cells. Cardiovascular Research, 94 (3), 439-449. 


\title{
5 \\ Comparative Analysis of Adult Stem Cell Niches
}

\author{
Bryan A. Fioret and Antonis K. Hatzopoulos \\ Department of Medicine, Vanderbilt University, USA
}

\subsection{Adult Stem Cells}

Adult stem cells (ASCs) are unique clusters of undifferentiated cells which reside in specialized tissue-specific niches within an adult organ. ASCs have the ability to selfrenew and to differentiate into some or all of the mature cells of the specific organ in which they reside [1]. They are also known as somatic stem cells, or stem cells of the body, to distinguish them from germ cells involved in reproduction.

\section{Box 5.1 Abbreviations and Acronyms}

ASC adult stem cell

BMP bone morphogenetic protein

EGF epidermal growth factor

HFSC hair follicle stem cell

HSC hematopoietic stem cell

IPC intermediate progenitor cell

ISC intestinal stem cell 


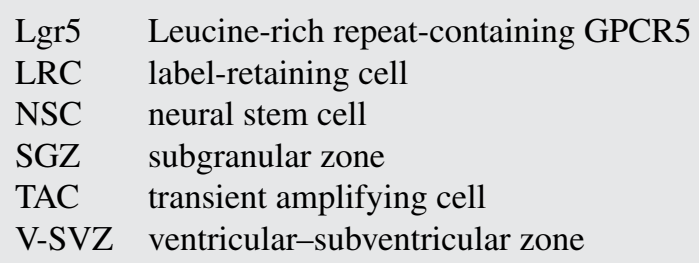

ASCs primarily differ from their embryonic stem cell (ESC) counterparts in both their origins and their lineage differentiation capabilities. ESCs, derived from the inner cell mass (ICM) of the blastocyst, are pluripotent and capable of differentiating into the three germ layers of the embryo (endoderm, mesoderm, and ectoderm) and generating all embryonic and somatic cell types [2]. Unlike ESCs, whose origins are clearly defined, the origins of various ASC populations are still unclear. The differentiation potential of ASCs is also more limited (multipotent instead of pluripotent) and they are generally lineage-restricted to the cell types of their host organ [3].

ASCs have been found in adolescent and adult animals, in tissues such as the hair follicles of the skin, intestine, bone marrow, specific regions of the brain, skeletal muscle, liver, teeth, ovaries, and testes [4]. However, ASC populations are relatively rare. Within each of these tissues, ASCs exist in specified niches, which are local microenvironments responsible for regulating their proliferation and differentiation [1,3]. ASCs often remain within their specific niche in a quiescent state until required to proliferate. ASC populations are maintained through symmetric cell divisions, in which two identical daughter stem cells are generated. They are also capable of generating multipotent progenitors with limited selfrenewal capacity through asymmetric division [3]. One of the primary characteristics of ASCs is their ability to self-renew indefinitely [3]. Consequently, they play a vital role in the maintenance of organ homeostasis by replacing dying cells lost to wear-and-tear, and also serve as a regenerative cell source after tissue damage or disease [3].

Because of their ability to self-renew and differentiate into mature cell types, ASCs are a potential resource for a wide variety of therapeutic applications. For example, various types of organ-specific ASC have been administered to animals and patients in an attempt to treat hematopoietic cancers [5], vascular disease [6], spinal cord injury [7], and cirrhosis of the liver [8]. The versatility and therapeutic potential of ASCs, as well as their need for maintenance and repair of adult tissue, warrant further study to optimize their use for treatment of tissue-specific disease or injury. The number of organs in which ASCs have been identified and characterized is continuously growing. However, numerous questions remain regarding their origins and the factors regulating their proliferation and differentiation. Consequently, significant effort has been applied toward understanding their regulatory signals and the regions in which they reside: the stem cell niche.

\subsection{Adult Stem Cell Niches}

The concept of a niche for cells was first proposed in 1978 based on studies involving hematopoietic stem cells (HSCs) [9]. Contradictory data indicated apparent limitless 
renewal of stem cells but a finite lifespan for spleen colony-forming cells, which were believed to be HSCs themselves [9]. To reconcile this discrepancy, the idea of a stem cell niche was proposed to explain how stem cells were regulated to continuously self-renew but also to generate differentiated progeny that do not persist indefinitely and have various rates of turnover.

The stem cell niche is more fully defined as a unique, tissue-specific, regulatory microenvironment responsible for enabling and controlling stem cell self-renewal while balancing internal and external molecular signals for the maintenance or repair of host tissue [1]. An increasing number of studies have identified a wide variety of tissue-specific stem cells, each with its own unique niche [3]. Studying the relationship between these stem cells and their niche environments will provide a better understanding of maintenance of organ homeostasis, or repair of damaged tissue in the adult.

The primary focus of this chapter is the study and comparison of four specific, wellcharacterized niches in mammalian adult organs. These niches are found within (i) the hair follicles of the skin, (ii) the crypts of the small intestine, (iii) marrow within the bone, and (iv) distinct neural regions of the brain. The role of the niche in providing structure and regulating resident stem-cell proliferation, maintenance, and differentiation through coordination of support cells and molecular signaling is also reviewed.

\subsection{The Hair Follicle Stem Cell (HFSC) Niche}

The skin is composed of three layers: the epidermis, the dermis, and a subcutaneous fatty layer, the hypodermis. The hair follicle, the structure which produces hair, extends through each of these layers. It is part of the pilosebaceous unit, which also includes the hair shaft, sebaceous gland, and arrector pili muscle. Each hair follicle contains a bulge region in the outer root sheath, which houses quiescent follicular stem cells [10]. The hair germ (HG) lies directly below the bulge and contains another cluster of proliferating follicular stem cells. Signals from the dermal papilla (DP), which lies at the base of each hair follicle, stimulate activation of HG stem cells. Hair follicle stem cells (HFSCs) reside in the bulge region and migrate to the HG to regenerate hair and sebaceous glands [11].

HFSCs exist in a quiescent state in the bulge region and in an active state in the HG and are capable of generating all lineages of hair $[10,12]$. Studies of HFSCs have progressed rapidly due to their location in the anatomically distinct bulge region and their ability to be isolated and studied in transplant models [13]. Their primary role is to serve as a source of cells for hair-follicle growth during the hair cycle.

The hair cycle is a three-phase process of anagen (growth), catagen (apoptosis-mediated retraction), and telogen (resting). Each hair follicle contains a permanent segment (including the bulge and sebaceous gland) and a cycling segment, which moves through the three haircycle phases $[10,14]$. Anagen begins when the DP is proximal to the bulge and HG, and stimulates HFSC differentiation from the HG through Wnt/ $\beta$-catenin signaling, fibroblast growth factor 7 (FGF7), and antagonism of bone morphogenetic protein (BMP) signaling [15]. Activated HG cells enter the hair matrix that surrounds the DP, which causes activation and migration of quiescent HFSCs in the bulge to replenish the lost HG cells. TACs derived from activated HG stem cells contribute to hair growth, which pushes the DP further from the bulge HFSCs. The physical separation of these two components minimizes the 
continued activation of HFSCs, allowing for the transition to catagen. During this phase, apoptosis of active progenitor cells then causes the hair follicle to shrink until the DP again rests close to the bulge and HG, allowing the cycle to start anew, after the resting period of telogen.

The hair follicle bulge serves as a niche for HFSCs. These cells were first hypothesized to be stem cells based on studies which identified slow-cycling cells within this anatomical compartment [16]. Labeling of HFSCs using either [3H]-thymidine or 5-bromo$2^{\prime}$-deoxyuridine (BrdU) demonstrated their existence as label-retaining cells (LRCs), but live cells could not be isolated. Subsequently, transgenic mice were generated with a keratin 5 (K5) promoter driving expression of a doxycycline-controlled, histone H2B-GFP fusion protein in the cells of the skin epithelium. Due to their slow cycling, bulge HFSCs retained the H2B-GFP after a 4-month chase and could be isolated [12]. The self-renewal and multipotency characteristics of HFSCs were further defined using keratin 14 (K14) promoter-driven GFP transgenic mice [17]. HFSCs isolated using GFP and their stem-cell marker CD34 generated the entire hair follicle after transplantation on to the back skins of nude mice.

mRNA analysis of HFSCs (isolated LRCs) compared to their differentiated progeny revealed a number of differences, which provide insight into their niche [12]. HFSCs had greater expression $(\geq 2 \times)$ of transcripts encoding cell-cycle regulatory proteins, including keratinocyte growth inhibitors, and downregulated levels of proliferation-related proteins (Ki67 and Cdc25C) [12]. Expression of both secretory and integral membrane proteins ( $\beta 1,4,6$ and $\alpha 6$ integrins, among others) were elevated in the HFSCs [12], including ephrin receptors and their ligands, which are involved in cell-cell communication and the formation of tissue boundaries. In addition, $\beta 6$ integrin is expressed only during the early anagen growth phase and may stimulate stem cell proliferation and migration through interaction with the extracellular matrix (ECM) glycoprotein tenascin C. These findings imply a level of organization within the bulge that allows for regulation of HFSC proliferation, adhesion, and interaction with neighboring cells.

The bulge HFSC niche is organized into upper and lower regions, each with a distinct subset of cells [10,17]. The stem-cell marker Leucine-rich repeat-containing GPCR5 (Lgr5) is expressed in the bulge and HG and can be used to subclassify these populations of cells. The upper bulge contains a population of $\mathrm{CD} 34^{+}, \mathrm{Lgr}^{-}$cells, while the lower bulge contains a $\mathrm{CD}_{4} 4^{+}, \mathrm{Lgr}^{+}$cell pool. Within the $\mathrm{HG}$, closer to the DP, is a population of $\mathrm{CD}^{-}{ }^{-}, \mathrm{Lgr}^{+}$cells. These are activated first by the DP during anagen to generate TACs and generate the hair shaft. The quiescent population of upper-bulge $\mathrm{CD} 34^{+}, \mathrm{Lgr}^{-}$cells is then activated to replace the HG cells. Thus, the bulge contains unique sites for maintaining quiescent and active populations of HFSCs, restricting their growth and differentiation until replenishment of cells is required.

The canonical Wnt and BMP signaling pathways are crucial regulators of the bulge HFSC niche. Transgenic mice with transiently stabilized $\beta$-catenin exhibited de novo hairfollicle morphogenesis, forming newly functioning follicles with DP, sebaceous gland, outer and inner root sheath, and hair shaft [18]. The interaction of $\beta$-catenin and the Wnt-dependent transcription factor Lef-1 was strongly implicated in mediating this new growth. Furthermore, transgenic mice with enhanced $\beta$-catenin showed decreased time spent in telogen in favor of a more rapid switch to the anagen growth phase of the hair cycle. 
Signaling through BMP receptor 1A (BMPR-1A) is necessary for the differentiation of $\mathrm{HG}$ progenitor cells within the inner root sheath and hair shaft [19]. BMPR-1A null animals exhibit defects in their hair follicles. Additionally, mice unable to express the BMP inhibitor Noggin lack expression of Lef-1, indicating an intrinsic link between canonical Wnt and BMP signaling. Crosstalk between these two pathways begins with Nogginmediated inhibition of BMP signaling, allowing for activation of $\mathrm{Wnt} / \beta$-catenin to stimulate proliferation of HFSCs. Thus, activation of $\mathrm{Wnt} / \beta$-catenin signaling leads to proliferation of HFSCs, while BMP signaling represses their activation through inhibition of Wnt [20]. Consequently, maintenance of quiescence in the HFSC niche of the bulge involves a "Wnt-off/BMP-on" balance of mediators, which is reversed at the DP to stimulate cell differentiation and follicle growth.

Interestingly, in studies of individuals with androgenetic alopecia (AGA), or common baldness, cytokeratin15 $\left(\mathrm{KRT}^{+} 5^{+}\right)$stem cells were still present in samples of bald scalp [21]. These quiescent $\mathrm{KRT}^{+} 5^{+}$cells were small in size and localized within the bulge. However, a population of larger, proliferative $\mathrm{CD} 200^{+} \mathrm{CD} 34^{+}$progenitors located next to the bulge was noticeably diminished. Within these bald-scalp samples, the size of the hair follicle was also significantly smaller, perhaps due to a defect in the conversion of HFSCs to their progenitors. The role of the HFSC niche as a factor in this situation cannot be understated, especially when the problem with generation of follicular progenitors may be a result of deficient signal transduction at the HFSC level. A better understanding of the molecular cues related to this process, which may be misregulated by the bulge HFSC niche, could directly benefit individuals with AGA.

\subsection{The Intestinal Stem Cell (ISC) Niche}

Digestion of food and absorption of nutrients occurs in the gastrointestinal (GI) tract, particularly the stomach and the small and large intestines. The mix of digestive enzymes from the stomach and pancreas and bile from the gallbladder creates an inhospitable environment for GI epithelial mucosa, which causes these cells to have some of the most rapid turnover rates of any organ in the body. Mature cells of the intestinal epithelium have an average lifespan of less than 1 week, and thus require a robust pool of intestinal stem cells (ISCs) for maintenance of intestinal homeostasis [22].

The ISC niche is located at the bottom of the intestinal Crypts of Lieberkühn (crypts), which are small recesses within the epithelial lining of the small intestine and colon. Both of these regions contain crypts, but only the small intestine contains villi [20,22]: epithelial protrusions from the intestinal wall composed of mature absorptive cells known as enterocytes and numerous secretory cells, including enteroendocrine, goblet, and tuft cells. Paneth cells are also secretory but remain in the crypt of the intestine to support maintenance of ISCs. The ISC niche generates all of the cells of the small intestine. It does so as they migrate upwards into the villi, maturing into the cell types needed to replace lost cells. The colon also contains an ISC niche within the crypts, but progenitor cells migrate apically into a smooth epithelium (instead of villi), which is optimized for absorbing water [22].

Two populations of ISCs exist within the crypt niche of the small intestine, both of which are multipotent and capable of self-renewal. The first, at the +4 position from the crypt 
base, is a quiescent population of slow-cycling LRCs [10]. This population constitutes 2-4 of 16 cells in the circumferential ring of the crypt and is marked specifically by Bmi-1, mTert, and Hopx [23,24]. The second ISC population is found at the bottom of the crypt, interspersed between supportive Paneth cells, and marked by Lgr5, CD133 (or Prom1), and Sox9 $[22,25]$. This fast-cycling group is also known as crypt basal columnar cells (CBCs), and like the first group is able to generate the entire crypt and villi.

While the full extent of the interactions between these two ISC populations (Bmi- ${ }^{+}$ LRCs and $\mathrm{Lgr}^{+} \mathrm{CBCs}$ ) is still being elucidated, it is known that they participate in a bidirectional relationship. In mice, complete ablation of $\mathrm{Lgr}^{+}$-expressing cells did not affect intestinal epithelial homeostasis [25]. In response to the loss of these cells, Bmi-1 ${ }^{+}$ cell proliferation increased, and lineage-tracing studies indicate that they compensated by generating $\mathrm{Lgr}^{+}$cells. Conversely, in vitro and in vivo studies have demonstrated that $\mathrm{Lgr}^{+}$cells can generate $\mathrm{Hopx}^{+}$cells at the +4 position of the crypt [26]. These studies also indicate that the quiescent $\mathrm{Bmi}-1^{+}$ISC population interconverts into rapidly cycling $\mathrm{CBCs}$, which further differentiate to repopulate to the villi. Conversely, the quiescent Bmi$1^{+}$cells can be replenished from the CBCs. Thus, distinct clusters of ISCs in the crypt niche appear to interact and renew one another.

Within the crypts, the ISC niche contains a number of support cells and structural components. Paneth cells are specialized daughter cells, derived from and interspersed between $\mathrm{Lgr}^{+}$CBCs. They secrete antimicrobial enzymes and peptides such as lysozyme and cryptins/defensins, respectively [27]. In addition to providing defense against foreign agents at the mucosal surface, $\mathrm{CD} 24^{+}$Paneth cells express epidermal growth factor (EGF), transforming growth factor alpha $(\mathrm{TGF} \alpha), \mathrm{Wnt} 3$, and Dll4, which promote proliferation and maintenance of ISCs through their respective signaling pathways [27]. Genetic removal of Paneth cells in vivo leads to the loss of $\mathrm{Lgr}^{+} \mathrm{CBCs}$, indicating their important supportive role in the crypts of the small intestine.

Maintaining intestinal homeostasis under a wide range of digestive conditions requires a number of signaling pathways to control cell fate and is mediated via niche support cells throughout the crypt and villus. The canonical Wnt/ $\beta$-catenin pathway is crucial for maintenance and proliferation of undifferentiated ISCs [28,29]. Paneth cells release Wnt ligands, which act on the Wnt receptors Frizzled (Frz) and LRP5/6 in neighboring cells. They also produce the Wnt agonist R-spondin, which also activates Wnt through the Lgr5 and LRP5/6 receptors.

In transgenic mice, inducible ablation of the $\beta$-catenin gene caused a loss of all intestinal epithelial cells within 6 days of induction, as well as a decrease in number and size of intestinal crypts [29]. Loss of $\beta$-catenin also inhibits ISC proliferation, partially through decreased expression of the $\mathrm{Wnt} / \beta$-catenin target gene $\mathrm{c}-m y c$, which induces ISC proliferation, and partially through increased expression of the cell-cycle inhibitor p21 [29]. In addition, loss of $T c f 7 l 2$, the gene encoding the Wnt transcription factor Tcf-4, causes death in postnatal mice [30]. Analysis of the intestine in these mice revealed the absence of proliferative crypts between villi, the entire intestinal epithelium consisting of differentiated, nonproliferative villus cells. Conversely, young transgenic mice expressing a dominant stable mutation of $\beta$-catenin revealed excessive ISC proliferation and adenomatous polyps [31]. Furthermore, loss of adenomatous polyposis coli (APC), a critical member of the $\beta$-catenin destruction complex, induces a progenitor-like state throughout the crypts of the intestine and formation of colorectal adenomas $[32,33]$. These studies indicate the strong 
influence of canonical Wnt signaling on promoting ISC proliferation and maintenance of self-renewal.

Much like canonical Wnt signaling, the Notch pathway enhances the pool of proliferative ISCs in the crypt [28]. The Notch target gene Hairy, an enhancer of split (Hes1), and the putative stem-cell marker Musashi-1 (Msi1) both mark CBCs and +4 cells within the crypt [34]. Through lineage-tracing studies of Notch signaling-positive cells, using the Notch1 Intramembrane Proteolysis ( $\mathrm{N}_{1} \mathrm{IP}-\mathrm{Cre}$ ) mouse line, Notch signaling was found to be active in ISCs [35]. Cell-fate studies in mice have also demonstrated that Notch1 and -2 receptors are specifically expressed in crypt stem cells but do not mark supportive Paneth cells [36].

Transgenic mice lacking the Notch-dependent transcription factor CSL display an expanded number of secretory goblet cells and less proliferative ISCs [22]. The same effect is observed after antibody blockade of the Notch1 receptor [37]. Furthermore, mice expressing a constitutively active Notch1 receptor (via the Notch intracellular domain, NICD) contain villi populated with immature progenitor cells and lack a differentiated epithelium [36]. These studies strongly indicate the role of Notch signaling in maintaining ISC proliferation and inhibiting differentiation to secretory cells. This maintenance of stemness via the Notch pathway is partially achieved in $\mathrm{Lgr}^{+} \mathrm{CBC}$ s through interaction with the Notch ligand Dll4 from supportive Paneth cells [27].

In addition to active Wnt and Notch signaling, the BMP pathway is active in the epithelium and mesenchyme of the small intestine [28]. Mouse models overexpressing the BMP inhibitor Noggin display ectopic crypts and have intraepithelial neoplasia commonly associated with polyposis [38]. A similar phenotype is observed in transgenic mice lacking the BMPR-1A (Alk3), which exhibit hyperproliferation of ISCs [39]. Thus, BMP signaling represses proliferation of ISCs, which reduces the number of aberrant crypts and polyp formation in the intestine. Suppression of ISC self-renewal via intestinal BMP signaling alternatively promotes ISC differentiation into secretory enteroendocrine cells.

BMP signaling also regulates the maturation of differentiated ISC progeny by counteracting the proliferative effects of the Wnt/ $\beta$-catenin pathway. BMP signaling activates the phosphatase PTEN, which then blocks AKT to inhibit activation of $\beta$-catenin [20]. This BMP-mediated suppression of canonical Wnt balances ISC self-renewal with differentiation, since both processes are equally necessary for intestinal homeostasis.

Isolated $\mathrm{Lgr}^{+} \mathrm{CBCs}$ have been successfully used to generate crypt-villus "minigut" structures in vitro [40]. Single $\mathrm{Lgr}^{+}$stem cells, isolated from the intestines of Lgr5-EGFPires-CreERT2 transgenic mice, were isolated based on expression of GFP and cultured in conditions promoting growth of intestinal epithelium. These conditions involved treatment with EGF, Noggin, and the Wnt agonist R-spondin to imitate the proliferative crypt environment. After 2 weeks of treatment, proliferative intestinal-like organoids formed from individual $\mathrm{Lgr}^{+} \mathrm{GFP}^{+}$cells. These structures naturally formed crypts with supportive Paneth cells interspersed between proliferating $\mathrm{Lgr}^{+} \mathrm{GFP}^{+}$ISCs and contained a lumen with a single-cell layer of villus-like epithelial cells. Amazingly, these organoid structures could be cultured for months while still maintaining crypt-villus morphology and proliferative ability.

The ability to generate and maintain intestinal organoids in vitro indicates our increasing knowledge of the ISC niche. A better understanding of the support cells, crypt structure, and molecular signaling pathways which regulate ISC proliferation and differentiation will help with the prevention and treatment of intestinal diseases, particularly cancerous growths. 


\subsection{The Hematopoietic Stem Cell (HSC) Niche}

In the adult human, approximately $10^{11}-10^{12}$ blood cells are generated every day [41]. Due to their short lifespan, blood cells are replenished by a constant source of multilineage progenitors, derived from self-renewing HSCs in the bone marrow. HSCs are responsible for establishing and regenerating all mature blood cells, including lymphoid cells (Band T-lymphocytes, natural killer cells) and myeloid cells (erythrocytes, megakaryocytes, monocytes, macrophages, neutrophils, etc.) [42].

Studies of HSCs have progressed rapidly due to the discovery of a plethora of stagespecific surface markers that are easily identified from monoclonal antibodies, their capacity for dye efflux, and their unique metabolic properties. Consequently, the multistep journey of HSC development and migration has been largely mapped using animal models. During development of the mammalian embryo, hematopoiesis occurs at specific sites in a temporally regulated manner. It first begins in the blood islands of the yolk sac, then moves to the aorta-gonad mesonephros (AGM) region surrounding the dorsal aorta, then to the placenta, fetal liver, spleen, and finally the bone marrow [42]. In each of these locations, the niche develops prior to seeding by the migratory HSCs, which circulate during hematopoiesis in the embryo [43]. Primitive HSCs gradually mature and expand into a definitive, quiescent HSC pool as the cells home from one niche to the next after expressing the appropriate receptors induced by the current niche $[3,44]$.

The events that occur during HSC development illustrate the diverse functions of their specific niches. Each site controls the formation of unique blood cell populations and regulates either expansion or differentiation of the corresponding HSCs [42]. The hemangioblast in the yolk sac generates endothelial cells and red blood cells (RBCs), while the AGM and placental niches induce formation of cycling HSCs from hemogenic endothelium. In the fetal liver, long-term HSCs (LT-HSCs) cycle rapidly to generate short-term HSCs (STHSCs), which give rise to all common myeloid and lymphoid progenitors. Within the adult, populations of LT-HSCs become quiescent and cycle slower but continue to form all mature hematopoietic lineages. By this stage, the adult HSC niche controls HSC self-renewal and differentiation to regulate homeostasis of the blood system.

\subsubsection{Endosteal Niche}

Within the bone marrow, the HSC niche consists of two general sites: (i) a quiescent, osteoblastic niche at the endosteum and a (ii) proliferative, vascular niche in the central marrow [45]. The osteoblast (OB) is the primary HSC support cell within the osteoblastic niche. OBs and osteoblastic lining cells reside along the endosteum, a connective tissue lining within the medullary cavity of the bone, and interact with HSCs. The positive role of OB support cells in regulating HSC proliferation becomes apparent when the transcriptional regulator of $\mathrm{OB}$ differentiation, Runx2, is removed. Runx2 -/- transgenic mice showed impaired bone marrow hematopoiesis due to reduced $\mathrm{OB}$ differentiation, resulting in a lack of niche sites for HSCs [46]. Furthermore, expansion of the number of OBs through activation of PTH/PTHrP receptors (PPRs) enhanced the expression of Jagged-1, which induced HSC proliferation through activation of Notch signaling [47]. Alternatively, OBs can release the glycoproteins osteopontin (OPN) and thrombopoietin (TPO) to block HSC proliferation and maintain quiescence within the endosteal niche [45]. 
In addition to HSC regulation by OBs, other support cells and inorganic ions serve crucial functions in the endosteal niche. CXC chemokine ligand 12 (CXCL12) is highly expressed in particular cells called CXCL12-abundant reticular (CAR) cells [48]. CAR cells in the endosteum maintain the quiescent HSC pool through signaling via its receptor, CXCR4. HSCs are also capable of sensing extracellular calcium $\left(\mathrm{Ca}^{2+}\right)$ concentrations through a $\mathrm{Ca}^{2+}$-sensing G-protein-coupled receptor (GPCR). In response to $\mathrm{Ca}^{2+}$ levels, HSCs localize next to OB support cells in the endosteal niche, which controls the location of hematopoiesis within the bone.

Osteoclasts (OCLs) and macrophages exert structural and regulatory roles within the endosteal niche. OCLs form cavities in the bone, in which HSCs reside. Mouse models with defective OCLs have greater HSC mobilization and peripheral localization, suggesting OCLs suppress HSC mobilization [49]. Similarly, a specific subset of endosteal F4/80 macrophages (termed osteomacs) are necessary for maintaining HSCs within the endosteal niche through their support of OBs. The addition of granulocyte colony-stimulating factor (G-CSF) causes a loss of osteomacs, suppresses OBs, and ultimately induces mobilization of peripheral HSCs through disruption of the CXCR4/CXCL12 pathway [50].

Maintenance of HSC quiescence within the endosteal niche is mediated by repression of the canonical Wnt signaling pathway through its inhibitor, secreted Frizzled-related protein 1 (sFRP-1) [10]. Inhibition of glycogen synthase kinase 3 (GSK3), a repressor of $\beta$-catenin, also induces HSC progenitor cell activity, indicating the proliferative effect of Wnt signaling on these cells. Alternatively, activation of BMP signaling through various pathway agonists is responsible for repressing HSC proliferation in this region of the niche.

\subsubsection{Vascular Niche}

To maintain blood cell homeostasis, quiescent LT-HSCs within the endosteal niche migrate to the sinusoids of the vascular niche in the central marrow and become rapidly cycling ST-HSCs. Sinusoids are fenestrated, capillary-like blood vessels which form the vascular component of the bone marrow and are the sites of entry and exit for circulating, HSCderived blood cells. Bone marrow sinusoidal endothelial cells (SECs) line the lumen of these vessels and express vascular-endothelial growth factor receptor-2 (VEGFR-2) [45]. Myeloablation of bone marrow through lethal irradiation destroys marrow cells. Bonemarrow transplantation is required for recovery. Blocking VEGFR-2, and thus SECs, during this time impedes regeneration and engraftment of HSCs, illustrating the direct role of bonemarrow SECs in supporting HSCs [51].

This SEC/HSC interaction is dependent, in part, on Notch signaling. Just as OBs express the Notch ligands Jagged-1 and -2, SECs regulate HSC proliferation through the same Notch-dependent pathway [45]. However, in vitro and in vivo studies suggest this pathway may only be activated under stressful conditions, such as after myeloablation. In addition, SECs regulate vascular HSCs through expression of the c-Kit receptor ligand stem-cell factor (SCF) [52]. SCF is important in maintaining vascular HSCs, since its removal from vascular endothelial cells causes the loss of HSCs. This type of SCF-mediated regulation of HSCs is specific to endothelial cells, as loss of SCF in OBs or hematopoietic cells does not affect HSC number or function. 
Further regulation in the vascular niche is achieved by a balance of canonical Wnt and BMP signaling. Activation of canonical Wnt occurs through nuclear localization of $\beta$ catenin, which induces proliferation of formerly quiescent HSCs. This process is balanced by inhibition of BMP signaling, which would otherwise repress cell division.

In addition to vascular SECs, a number of perivascular cells are required for HSC maintenance. Perivascular CAR cells, as distinct from the CAR cells of the endosteum, provide structural support to the sinusoids at the central marrow and form a barrier between the bone-marrow stroma and circulation [45]. These cells also provide a supportive role to HSCs within the perivascular space and express SCF and CXCL-12 (or stromal cell-derived factor 1).

A second type of perivascular support cell in the central marrow is the Nestin ${ }^{+}$mesenchymal stem cells (MSCs). Considered to be a subgroup of CAR cells, Nestin ${ }^{+}$MSCs localize near HSCs and express genes involved in their maintenance, such as Cxcl-12, c-kit ligand, interleukin-7, vascular cell adhesion molecule 1, and osteopontin [53]. Diptheria toxin-mediated depletion of Nestin ${ }^{+}$MSCs detrimentally affects HSC number and homing to the bone marrow, indicating these cells' supportive role for HSCs.

Nonmyelinating Schwann cells also have an important role in regulating HSC quiescence and activity within the vascular niche. These glial cells ensheath autonomic nerves that innervate the bone-marrow vasculature and modify inactive TGF $\beta$, released by a number of nearby cells, into an active form that initiates signaling through Smad [54]. Loss of Schwann cells through autonomic nerve denervation and TGF $\beta$ R-2 deficient HSCs both independently caused loss of HSCs. These losses were due to defects in TGF $\beta / \mathrm{Smad}$ signaling and the inability to regulate HSC quiescence.

\subsubsection{Progeny "Niche"}

An additional level of control over the HSC niche is provided by feedback regulation via progenitor cells. Bone-marrow $\mathrm{CD}_{169^{+}}$macrophages, derived from common myeloid progenitors (CMPs), interact with $\mathrm{Nestin}^{+}$vascular cells to retain HSCs within the niche [45]. Megakaryocytes, CMP progenitors that generate platelets, release TGF $\beta$ and Angiopoietin1 (Ang-1) to decrease HSC proliferation [45]. Finally, an abnormally high number of platelets will deplete TPO, which stimulates a negative feedback loop involving megakaryocytes to ultimately induce HSC quiescence within the bone marrow [55].

\subsection{The Neural Stem Cell (NSC) Niche}

The adult brain was initially believed to be a postmitotic organ, unable to regenerate its parenchymal cells, or neurons. However, while neurogenesis occurs during development of the embryo in transient niches, it has been unexpectedly shown to persist in specific areas of the brain after birth and throughout adult life. Adult neurogenesis has best been characterized within neural stem cell (NSC) niches located in the ventricular-subventricular zone (V-SVZ) of the lateral ventricles and the subgranular zone (SGZ) of the dentate gyrus [56]. Other regions of the brain such as the striatum, substantia nigra, amygdala, and neocortex have been proposed as sites of neurogenesis but are currently debated. NSC markers commonly used to label these populations include the intermediate filament proteins Nestin and 
glial fibrillary acidic protein (GFAP), the RNA-binding proteins Musashi-1 and -2, and the transcription factor Sox2 [56].

\subsubsection{V-SVZ Niche}

During neural development, radial glial cells give rise to a group of astroglial-like cells, known as B1 cells, which persist in the adult brain and function as NSCs [57]. Neurogenesis within the V-SVZ utilizes these B1 cells, located in the walls of the lateral ventricles, which extend through this region to contact blood vessels within the SVZ [57]. B1 cells are induced to generate a subpopulation of transient amplifying cells (TACs), also known as intermediate progenitor cells (IPCs) or type-C cells. These TACs divide briefly and then become neuroblasts (type-A cells), which, in rodents, migrate along the rostral migratory stream to the olfactory bulb and mature into interneurons [58].

Understanding the structure of V-SVZ B1 cells provides insight into the role of their support cells and regulatory factors. B1 cells have three domains (apical, intermediate, and distal), which extend from the ventricular lumen through the ventricular zone (VZ) and SVZ. The apical side of B1 cells contains a primary cilium, which projects through the ventricular wall to contact the cerebrospinal fluid (CSF) within the lateral ventricles. This cilium is surrounded in a circular manner by ependymal cells, a simple cuboidal epithelium, with apical cilia that circulate the CSF within the ventricular lumen [59]. Ependymal cells provide a supportive role by attaching to $\mathrm{B} 1$ cells at intercellular junctions and exposing them to the CSF.

Exposure of B1 cells to the CSF introduces soluble factors and signaling molecules that can regulate their proliferation. Insulin-like growth factor 2 (IGF2), circulating with the CSF, binds to the IGF1 receptor (IGF1R) on the apical surface of B1 cells and promotes proliferation [60]. B1 cell proliferation is also induced through the release of the BMP antagonist Noggin from ependymal cells and by sequestration of the BMP4 ligand [57]. In addition, Sonic hedgehog $(\mathrm{SHH})$ signaling mediators may regulate ventral specification through the primary cilium, and a number of Wnt ligands have been shown to exist near the surface of the ventricles [57]. Correspondingly, increased expression of the Wnt receptor Frizzled has been observed in ventricular progenitor cells. Thus, the CSF contains numerous secreted factors which regulate NSC survival and proliferation within the lateral ventricles.

Regulation of B1 NSC proliferation through negative-feedback mechanisms is likely to occur within the intermediate domain. In this region, B1 cells maintain cell-cell contacts with their progenitors (TACs and neuroblasts) and the neuronal terminals of support cells [57]. TACs express the bHLH transcription factor ASCL1 (Mash1), which promotes the production of Notch ligands. Activation of canonical Notch signaling then represses generation of TACs through the downstream mediator HES1, illustrating a mechanism of NSC maintenance. Furthermore, neuroblasts release the neurotransmitter gamma-aminobutyric acid (GABA), which represses TAC proliferation by blocking progression through the cell cycle (via depolarization). Alternatively, release of the neurotransmitter dopamine from neighboring neuronal terminals of the substantia nigra can stimulate TAC proliferation through release of EGF [61].

Like the primary cilium of the apical region, which receives input from the CSF, the distal domain of $\mathrm{B} 1$ cells is in contact with factors of the bloodstream. A basal extension reaches into the SVZ and surrounds the blood vessels of its vascular plexus using a unique 
"end-foot" process [57]. Interaction with both blood-vessel endothelial cells and bloodderived factors affects B1 cell proliferation. In summary, the structure of B1 NSCs of the $\mathrm{V}-\mathrm{SVZ}$ allows them to receive regulatory cues from several environments (CSF, progenitors, blood-vessel endothelial cells, and blood) which regulate maintenance and proliferation within the walls of the lateral ventricles.

\subsubsection{SGZ Niche}

A second NSC niche exists in the SGZ of the dentate gyrus, within the hippocampus [57]. The dentate gyrus is composed of several layers, with the SGZ located at the bottom, directly above the hilus (a structure where blood vessels and nerves enter). The granule cell layer (GCL) and inner molecular layer (IML) exist above the SGZ. In the SGZ, astroglial cells, known as radial astrocytes (RAs) or type-1 progenitors, serve as the NSC. When induced, RAs generate transient amplifying progenitors known as $\mathrm{IPC}_{1}$ and $\mathrm{IPC}_{2}$ (or type $2 \mathrm{a}$ and $2 \mathrm{~b}$, respectively). Once generated, $\mathrm{IPC}_{1}$ cells quickly develop into proliferating $\mathrm{IPC}_{2}$ cells, characterized by expression of the early neuronal marker doublecortin [62]. $\mathrm{IPC}_{2}$ cells then develop into immature granule cells (IGCs), which become mature granule cells (GCs) and extend axons into both the hilus and inner molecular layer of the dentate gyrus.

Like the B1 cells of the V-SVZ, the RAs of the SGZ can be segmented into three distinct domains (proximal, intermediate, and distal), each with unique functions [57]. The proximal domain of an RA is located within the SGZ and contains lateral processes that extend from the soma to contact nearby vasculature and neighboring RAs. This domain is in direct contact with the hilus and contains a primary cilium expressing smoothened receptors for regulation of proliferation through $\mathrm{SHH}$ signaling. The proximity of the RA proximal domain to the vasculature within the hilus also allows for stimulation of neuronal proliferation via release of IGF and vascular-endothelial growth factor (VEGF) [63]. These growth factors are crucial for RA neurogenesis and maintenance of synaptic plasticity, illustrating the crucial role of endothelial support cells. In addition to stimulating proliferation, supporting vascular endothelial cells release brain-derived neurotrophic factor (BDNF) to induce differentiation of RA progenitors [64].

The intermediate domain of RAs contains the soma and main axonal shaft, which projects through the granule cell layer, contacting IPCs and mature GCs [57]. These interactions allow for maintenance of RAs. Specifically, canonical Notch signaling regulates expression of SOX2, a transcription factor that prevents activation of the neuronal fate mediator NeuroD [65]. Blocking expression of NeuroD preserves the pool of RAs, preventing their generation of IPCs. This type of control over RA proliferation, as well as of ASCL1 ${ }^{+}$IPC $_{1}$ cell proliferation, is governed by Notch signaling, equivalent to the feedback mechanisms regulating $\mathrm{B} 1$ cells.

Further regulation at the intermediate domain of RAs occurs through mediation of BMP signaling via expression of Noggin. This BMP antagonist is expressed within the hilus and dentate gyrus, and its overexpression induces RA proliferation [66]. Thus, canonical BMP signaling may maintain the current RA population by promoting a quiescent state. In direct contrast to the quiescent state invoked by BMP signaling, release of Wnt3 from astrocytes stimulates differentiation of NSCs within the SGZ. In addition, contact of the RA axonal shaft with mature GCs may lead to NSC proliferation through 
detection of neuronal activity via neurotransmitters. This type of RA stimulation is involved with long-term potentiation (LTP), a process necessary for learning and memory in the hippocampus [67].

The distal domain of RAs extends into the inner molecular layer of the dentate gyrus and forms a terminal arbor. Here, the thin radial processes are influenced by neurotransmitters such as GABA and Glutamate, released from interneurons and Mossy cells, to generate IPCs from differentiated RAs [68].

Overall, precise regulation of RAs occurs through interaction of their three general domains with progenitors, hippocampal support cells, and signaling mediators. These types of refined interaction allow for maintenance of the RA NSC population within their SGZ niche, as well as formation of the mature GCs involved in neuronal plasticity.

\subsection{A Comparison between Tissue-Specific Adult Stem Cell Niches}

Each of the ASC niches discussed here incorporates similar concepts in the regulation of its respective stem cells. Several of these niche similarities are observed in different organs, and they are also a broadly conserved evolutionarily theme between vertebrates and invertebrates, such as D. melanogaster and C. elegans [1]. In general, maintenance of ASCs occurs through regulation of quiescent (slow-cycling) and active (rapidly proliferating) populations. Support cells within the niche control these opposing processes through release of various mediators, which stimulate or inhibit stem-cell proliferation and differentiation. Niche stem cells are responsive to secreted factors through various receptors and when induced to proliferate either generate identical daughter cells or precursors destined to become mature cells of the organ. This niche-mediated regulation of ASCs is crucial for maintenance of the tissue and repair after damage.

The general use of stromal support cells that release various factors to influence stem cell renewal extends throughout the mammalian niches discussed previously. Studies in both mice and rats show that a variety of niche support cells regulate their respective stem cells through a balance of Wnt and BMP pathway mediators. Interestingly, these signaling pathways are highly conserved within the skin, small intestine, bone marrow, and brain (lateral ventricle and hippocampus), and often elicit similar effects regardless of the tissuespecific niche [3]. For example, within the small intestine, at the base of the crypts, support cells release canonical Wnt signaling mediators to promote ISC proliferation [28,29]. HSCs within the bone marrow directly stimulate their self-renewal through autocrine signaling via the Wnt pathway [69]. Wnt signaling also promotes proliferation of bulge stem cells within the hair follicle $[18,70]$. However, the stimulatory effects of $\mathrm{Wnt} / \beta$-catenin also require inhibition of BMP signaling, which is often achieved through expression of Noggin. Alternatively, active BMP signaling can maintain stem cells in a quiescent state in each of these niches.

Similar structural features also exist between ASC niches. Cell-cell and cell-ECM adhesion is necessary to retain quiescent stem cells within their niche, or to orient proliferating cells in a manner which induces symmetrical or asymmetrical division. A variety of adherens junctions and integrins provide cell-cell contact or cell attachment to a basal lamina [3]. HSCs and their OB support cells anchor to each other through $\mathrm{N}$-cadherin junctions and attach to their respective basal lamina through $\beta 1$-integrins [71]. Targeted deletion of 
$\beta 1$-integrin within hair-follicle keratinocyte stem cells disrupts keratinocyte proliferation, differentiation, and hair-follicle morphogenesis [72]. Finally, lack of the ECM glycoprotein tenascin $\mathrm{C}$ in the subventricular zone of the lateral ventricles detrimentally affects the function and number of NSCs [73]. These studies of three unique ASC niches indicate the general requirement that stem cell adhesion support cells and structural components for proper maintenance of the niche.

The structural architecture of individual stem cell niches also spatially separates subpopulations of cells for distinct regulation. For example, the location of stem cells within the hair follicle bulge allows for activation of specific transcriptional programs of gene expression. Depending on their state of proliferation, HFSCs upregulate unique combinations of mRNAs for control of cell adhesion, changes to cytoskeletal structure, or induction of cell growth [17]. Interestingly, a number of these mRNAs upregulated in bulge stem cells are also expressed in NSCs and HSCs to induce similar structural and metabolic changes, as well as to regulate cell growth. Within the intestine, separation of the two ISC populations allows for regulation of cell proliferation. A similar theme is observed in the bone marrow, in which slow-cycling endosteal niche stem cells replenish more rapidly cycling vascular progenitors.

An additional level of stem cell regulation is provided through soluble mediators (proteins and small molecules) released from progenitor cells. Progenitor-cell negative feedback represses NSC proliferation in both the subventricular and subgranular zones, as well as growth of ISCs and HSCs of the intestine and central marrow, respectively. Within the hair follicle bulge, migration of activated HG cells into the hair matrix induces the proliferation of quiescent HFSCs. Signaling molecules circulating in the bloodstream are also used to regulate stem cells. The common role of vasculature within each of the niches discussed allows for precise regulation of stem-cell activity through exposure to a variety of molecular mediators. In the brain, the proximal domain of RAs and the distal domain of B1 NSCs directly contact blood vessels. Consequently, bloodborne mediators regulate their proliferation state through interaction with membrane-bound receptors on both cells. Lastly, neuronal innervation of stem or progenitor cells can be employed to control maintenance of stem cell populations, as observed in both RAs and B1 NSCs.

The information learned from studies of stem cell niches in the hair follicle, intestine, bone marrow, and regions of the brain can be extrapolated to predict characteristics of stem cell niches in other organs of the body. Historically, the adult heart was believed to be composed of terminally differentiated, postmitotic cells, but a rising number of studies suggest the existence of cardiac stem cell (CSC) populations [74]. However, each putative population has a distinct expression of surface markers, unique or unknown origins, and varied cardiogenic potential. While it is becoming increasingly evident that CSC populations generate new cardiomyocytes in the heart, there is still considerable debate about the actual regenerative ability of the myocardium over the lifetime of an individual.

A similar challenge also exists in the identification and confirmation of an ASC population in the lungs. As in the adult heart, putative lung stem cell populations are believed to contribute lung epithelial cells for maintenance of the tissue, but fail to provide adequate regenerative ability after injury [75]. Current studies suggest that potential lung stem cell niches harbor a variety of pulmonary progenitors. However, characterization of these cells based on specific surface markers and demonstration of true stem-cell properties through direct in vivo studies are still lacking. Nevertheless, studies of confirmed stem cell niches 
from other organs within the body provide a solid model for studying the origin, location, structure, and regulation of novel, putative cardiac and pulmonary niches.

\section{$5.8 \quad$ Future Challenges}

While many questions about ASCs and their respective niches have been answered, several uncertainties remain. ASC niches within the hair, intestine, and bone marrow explain the source of replenishment for the millions of these cells that are lost every day. The discovery of niches within the brain occurred later, and regions of neurogenesis in addition to the V-SVZ and SGZ are currently under debate. Increasing evidence indicates that other organs, such as the heart and lungs, may also contain resident populations of ASCs. However, these putative cell populations must be definitively located and characterized in order to establish consensus within the field. In each of these cases, it will be necessary to understand the source and location of putative stem cell populations and how the integration of different signals culminates to affect their function and turnover within the niche. Finally, studies to address how and why ASCs and their niches change during normal aging processes, after injury and disease, will provide insight into the provision of improved care for an aging population.

\section{Acknowledgements}

This work was supported by an American Heart Association Fellowship to BAF and by NIH grants U01HL100398 and R01HL083958 to AKH.

\section{References}

(1) Scadden, D.T. (2006) The stem-cell niche as an entity of action. Nature, 441 (7097), 1075-1079.

(2) Thomson, J.A., Itskovitz-Eldor, J., Shapiro, S.S. et al. (1998) Embryonic stem cell lines derived from human blastocysts. Science, 282 (5391), 1145-1147.

(3) Jones, D.L. and Wagers, A.J. (2008) No place like home: anatomy and function of the stem cell niche. Nature Reviews Molecular Cell Biology, 9 (1), 11-21.

(4) National Institutes of Health (n.d.) What are adult stem cells? Available from: http://stemcells .nih.gov/info/basics/pages/basics4.aspx (last accessed March 7, 2014).

(5) Slavin, S., Nagler, A., Naparstek, E. et al. (1998) Nonmyeloablative stem cell transplantation and cell therapy as an alternative to conventional bone marrow transplantation with lethal cytoreduction for the treatment of malignant and nonmalignant hematologic diseases. Blood, 91 (3), 756-763.

(6) Subrammaniyan, R., Amalorpavanathan, J., Shankar, R. et al. (2011) Application of autologous bone marrow mononuclear cells in six patients with advanced chronic critical limb ischemia as a result of diabetes: our experience. Cytotherapy, 13 (8), 993-999.

(7) Lu, P., Jones, L., Snyder, E., and Tuszynski, M. (2003) Neural stem cells constitutively secrete neurotrophic factors and promote extensive host axonal growth after spinal cord injury. Experimental Neurology, 181 (2), 115-129.

(8) Terai, S., Ishikawa, T., Omori, K. et al. (2006) Improved liver function in patients with liver cirrhosis after autologous bone marrow cell infusion therapy. Stem Cells, 24 (10), 2292-2298.

(9) Schofield, R. (1978) The relationship between the spleen colony-forming cell and the haemopoietic stem cell. Blood Cells, 4 (1-2), 7-25. 
(10) Li, L. and Clevers, H. (2010) Coexistence of quiescent and active adult stem cells in mammals. Science, 327 (5965), 542-545.

(11) Alonso, L. and Fuchs, E. (2003) Stem cells of the skin epithelium. Proceedings of the National Academy of Sciences of the United States of America, 100, 11830-11835.

(12) Tumbar, T. (2004) Defining the epithelial stem cell niche in skin. Science, 303 (5656), 359-363.

(13) Kaur, P. (2006) Interfollicular epidermal stem cells: identification, challenges, potential. Journal of Investigative Dermatology, 126 (7), 1450-1458.

(14) Niemann, C. and Watt, F.M. (2002) others. Designer skin: lineage commitment in postnatal epidermis. Trends in Cell Biology, 12 (4), 185-192.

(15) Greco, V., Chen, T., Rendl, M. et al. (2009) A two-step mechanism for stem cell activation during hair regeneration. Cell Stem Cell, 4 (2), 155-169.

(16) Cotsarelis, G., Sun, T.T., and Lavker, R.M. (1990) Label-retaining cells reside in the bulge area of pilosebaceous unit: implications for follicular stem cells, hair cycle, and skin carcinogenesis. Cell, 61 (7), 1329-1337.

(17) Blanpain, C., Lowry, W.E., Geoghegan, A. et al. (2004) Self-renewal, multipotency, and the existence of two cell populations within an epithelial stem cell niche. Cell, 118 (5), 635-648.

(18) Gat, U., Das Gupta, R., Degenstein, L., and Fuchs, E. (1998) De novo hair follicle morphogenesis and hair tumors in mice expressing a truncated $\beta$-catenin in skin. Cell, 95 (5), 605-614.

(19) Kobielak, K., Pasolli, H.A., Alonso, L. et al. (2003) Defining BMP functions in the hair follicle by conditional ablation of BMP receptor IA. The Journal of Cell Biology, 163 (3), 609-623.

(20) Moore, K.A. and Lemischka, I.R. (2006) Stem cells and their niches. Science, 311 (5769), $1880-1885$

(21) Garza, L.A., Yang, C.-C., Zhao, T. et al. (2011) Bald scalp in men with androgenetic alopecia retains hair follicle stem cells but lacks CD200-rich and CD34-positive hair follicle progenitor cells. Journal of Clinical Investigation, 121 (2), 613-622.

(22) Jiang, H. and Edgar, B.A. (2012) Intestinal stem cell function in Drosophila and mice. Current Opinion in Genetics \& Development, 22 (4), 354-360.

(23) Sangiorgi, E. and Capecchi, M.R. (2008) Bmil is expressed in vivo in intestinal stem cells. Nature Genetics, 40 (7), 915-920.

(24) Potten, C.S., Booth, C., and Pritchard, D.M. (1997) The intestinal epithelial stem cell: the mucosal governor. International Journal of Experimental Pathology, 78 (4), 219-243.

(25) Tian, H., Biehs, B., Warming, S. et al. (2011) A reserve stem cell population in small intestine renders Lgr5-positive cells dispensable. Nature, 478 (7368), 255-259.

(26) Takeda, N., Jain, R., LeBoeuf, M.R. et al. (2011) Interconversion between intestinal stem cell populations in distinct niches. Science, 334 (6061), 1420-1424.

(27) Sato, T., van Es, J.H., Snippert, H.J. et al. (2011) Paneth cells constitute the niche for Lgr5 stem cells in intestinal crypts. Nature, $\mathbf{4 6 9}$ (7330), 415-418.

(28) Vanuytsel, T., Senger, S., Fasano, A., and Shea-Donohue, T. (2012) Major signaling pathways in intestinal stem cells. Biochimica et Biophysica Acta, 1830 (2), 2410-2426.

(29) Fevr, T., Robine, S., Louvard, D., and Huelsken, J. (2007) Wnt/ $\beta$-catenin is essential for intestinal homeostasis and maintenance of intestinal stem cells. Molecular and Cellular Biology, 27 (21), 7551-7559.

(30) Korinek, V., Barker, N., Moerer, P. et al. (1998) Depletion of epithelial stem-cell compartments in the small intestine of mice lacking Tcf-4. Nature Genetics, 19 (4), 379-383.

(31) Harada, N., Tamai, Y., Ishikawa, T. et al. (1999) Intestinal polyposis in mice with a dominant stable mutation of the $\beta$-catenin gene. The EMBO Journal, 18 (21), 5931-5942.

(32) Sansom, O.J., Reed, K.R., Hayes, A.J. et al. (2004) Loss of Apc in vivo immediately perturbs Wnt signaling, differentiation, and migration. Genes \& Development, 18 (12), 1385-1390.

(33) Shibata, H., Toyama, K., Shioya, H. et al. (1997) Rapid colorectal adenoma formation initiated by conditional targeting of the Apc gene. Science, 278 (5335), 120-123.

(34) Kayahara, T., Sawada, M., Takaishi, S. et al. (2003) Candidate markers for stem and early progenitor cells, Musashi-1 and Hes1, are expressed in crypt base columnar cells of mouse small intestine. FEBS Letters, 535 (1-3), 131-135.

(35) Vooijs, M., Ong, C.-T., Hadland, B. et al. (2007) Mapping the consequence of Notch1 proteolysis in vivo with NIP-CRE. Development (Cambridge, England), 134 (3), 535-544. 
(36) Fre, S., Huyghe, M., Mourikis, P. et al. (2005) Notch signals control the fate of immature progenitor cells in the intestine. Nature, 435 (7044), 964-968.

(37) Wu, Y., Cain-Hom, C., Choy, L. et al. (2010) Therapeutic antibody targeting of individual Notch receptors. Nature, 464 (7291), 1052-1057.

(38) Haramis, A.-P.G., Begthel, H., van den Born, M. et al. (2004) De novo crypt formation and juvenile polyposis on BMP inhibition in mouse intestine. Science, 303 (5664), 1684-1686.

(39) He, X.C., Zhang, J., Tong, W.-G. et al. (2004) BMP signaling inhibits intestinal stem cell self-renewal through suppression of Wnt-beta-catenin signaling. Nature Genetics, 36 (10), $1117-1121$.

(40) Sato, T., Vries, R.G., Snippert, H.J. et al. (2009) Single Lgr5 stem cells build crypt-villus structures in vitro without a mesenchymal niche. Nature, 459 (7244), 262-265.

(41) Cantani, A. (2008) Chapter one - immunology, in Cantani, A., Pediatric Allergy, Asthma and Immunology, Springer; pp. 1-219. Available from: http://link.springer.com. proxy.library.vanderbilt.edu/chapter/10.1007/978-3-540-33395-1_1 (last accessed March 7, 2014).

(42) Orkin, S.H. and Zon, L.I. (2008) Hematopoiesis: an evolving paradigm for stem cell biology. Cell, 132 (4), 631-644.

(43) Wright, D.E. (2001) Physiological migration of hematopoietic stem and progenitor cells. Science, 294 (5548), 1933-1936.

(44) Kyba, M., Perlingeiro, R.C.R., and Daley, G.Q. (2002) HoxB4 confers definitive lymphoidmyeloid engraftment potential on embryonic stem cell and yolk sac hematopoietic progenitors. Cell, 109 (1), 29-37.

(45) Nakamura-Ishizu, A. and Suda T. (2012) Hematopoietic stem cell niche: an interplay among a repertoire of multiple functional niches. Biochimica et Biophysica Acta (BBA) - General Subjects. Available from: http://linkinghub.elsevier.com/retrieve/pii/S0304416512002498 (last accessed March 7, 2014).

(46) Wang, C.Q., Jacob, B., Nah, G.S.S., and Osato, M. (2010) Runx family genes, niche, and stem cell quiescence. Blood Cells, Molecules, and Diseases, 44 (4), 275-286.

(47) Calvi, L.M., Adams, G.B., Weibrecht, K.W. et al. (2003) Osteoblastic cells regulate the haematopoietic stem cell niche. Nature, 425 (6960), 841-846.

(48) Sugiyama, T., Kohara, H., Noda, M., and Nagasawa, T. (2006) Maintenance of the hematopoietic stem cell pool by CXCL12-CXCR4 chemokine signaling in bone marrow stromal cell niches. Immunity, 25 (6), 977-988.

(49) Miyamoto, K., Yoshida, S., Kawasumi, M. et al. (2011) Osteoclasts are dispensable for hematopoietic stem cell maintenance and mobilization. Journal of Experimental Medicine, 208 (11), 2175-2181.

(50) Winkler, I.G., Sims, N.A., Pettit, A.R. et al. (2010) Bone marrow macrophages maintain hematopoietic stem cell (HSC) niches and their depletion mobilizes HSCs. Blood, 116 (23), 4815-4828.

(51) Hooper, A.T., Butler, J.M., Nolan, D.J. et al. (2009) Engraftment and reconstitution of hematopoiesis is dependent on VEGFR2 mediated regeneration of sinusoidal endothelial cells. Cell Stem Cell, 4 (3), 263-274.

(52) Ding, L., Saunders, T.L., Enikolopov, G., and Morrison, S.J. (2012) Endothelial and perivascular cells maintain haematopoietic stem cells. Nature, 481 (7382), 457-462.

(53) Méndez-Ferrer, S., Michurina, T.V., Ferraro, F. et al. (2010) Mesenchymal and haematopoietic stem cells form a unique bone marrow niche. Nature, 466 (7308), 829-834.

(54) Yamazaki, S., Ema, H., Karlsson, G. et al. (2011) Nonmyelinating Schwann cells maintain hematopoietic stem cell hibernation in the bone marrow niche. Cell, 147 (5), 1146-1158.

(55) de Graaf, C.A., Kauppi, M., Baldwin, T. et al. (2010) Regulation of hematopoietic stem cells by their mature progeny. Proceedings of the National Academy of Sciences of the United States of America, 107 (50), 21689-21694.

(56) Decimo, I., Bifari, F., Krampera, M., and Fumagalli, G. (2012) Neural stem cell niches in health and diseases. Current Pharmaceutical Design, 18 (13), 1755-1783.

(57) Fuentealba, L.C., Obernier, K., and Alvarez-Buylla, A. (2012) Adult neural stem cells bridge their niche. Cell Stem Cell, 10 (6), 698-708. 
(58) Kriegstein, A. and Alvarez-Buylla, A. (2009) The glial nature of embryonic and adult neural stem cells. Annual Review of Neuroscience, 32, 149-184.

(59) Mirzadeh, Z., Merkle, F.T., Soriano-Navarro, M. et al. (2008) Neural stem cells confer unique pinwheel architecture to the ventricular surface in neurogenic regions of the adult brain. Cell Stem Cell, 3 (3), 265-278.

(60) Lehtinen, M.K., Zappaterra, M.W., Chen, X. et al. (2011) The cerebrospinal fluid provides a proliferative niche for neural progenitor cells. Neuron, 69 (5), 893-905.

(61) O'Keeffe, G.C., Tyers, P., Aarsland, D. et al. (2009) Dopamine-induced proliferation of adult neural precursor cells in the mammalian subventricular zone is mediated through EGF. Proceedings of the National Academy of Sciences of the United States of America, 106 (21), 8754-8759.

(62) Lugert, S., Vogt, M., Tchorz, J.S. et al. (2012) Homeostatic neurogenesis in the adult hippocampus does not involve amplification of Ascl1(high) intermediate progenitors. Nature Communications, 3, 670 .

(63) Licht, T., Goshen, I., Avital, A. et al. (2011) Reversible modulations of neuronal plasticity by VEGF. Proceedings of the National Academy of Sciences of the United States of America, 108 (12), 5081-5086.

(64) Chen, J., Zacharek, A., Zhang, C. et al. (2005) Endothelial nitric oxide synthase regulates brainderived neurotrophic factor expression and neurogenesis after stroke in mice. The Journal of Neuroscience, 25 (9), 2366-2375.

(65) Kuwabara, T., Hsieh, J., Muotri, A. et al. (2009) Wnt-mediated activation of NeuroD1 and retro-elements during adult neurogenesis. Nature Neuroscience, 12 (9), 1097-1105.

(66) Bonaguidi, M.A., Wheeler, M.A., Shapiro, J.S. et al. (2011) In vivo clonal analysis reveals self-renewing and multipotent adult neural stem cell characteristics. Cell, 145 (7), 1142-1155.

(67) Bruel-Jungerman, E., Davis, S., Rampon, C., and Laroche, S. (2006) Long-term potentiation enhances neurogenesis in the adult dentate gyrus. The Journal of Neuroscience, 26 (22), 5888 5893.

(68) Förster, E., Zhao, S., and Frotscher, M. (2006) Laminating the hippocampus. Nature Reviews Neuroscience, 7 (4), 259-268.

(69) Reya, T., Duncan, A.W., Ailles, L. et al. (2003) A role for Wnt signalling in self-renewal of haematopoietic stem cells. Nature, 423 (6938), 409-414.

(70) Nguyen, H., Rendl, M., and Fuchs, E. (2006) Tcf3 governs stem cell features and represses cell fate determination in skin. Cell, 127 (1), 171-183.

(71) Wagers, A.J., Allsopp, R.C., and Weissman, I.L. (2002) Changes in integrin expression are associated with altered homing properties of Lin(-/lo)Thy1.1(lo)Sca-1(+)c-kit $(+)$ hematopoietic stem cells following mobilization by cyclophosphamide/granulocyte colony-stimulating factor. Experimental Hematology, 30 (2), 176-185.

(72) Brakebusch, C., Grose, R., Quondamatteo, F. et al. (2000) Skin and hair follicle integrity is crucially dependent on $\beta 1$ integrin expression on keratinocytes. The EMBO Journal, 19 (15), 3990-4003.

(73) Garcion, E., Halilagic, A., and Faissner, A. (2004) ffrench-constant C. Generation of an environmental niche for neural stem cell development by the extracellular matrix molecule tenascin C. Development (Cambridge, England), 131 (14), 3423-3432.

(74) Anversa, P., Kajstura, J., Rota, M., and Leri, A. (2013) Regenerating new heart with stem cells. Journal of Clinical Investigation, 123 (1), 62-70.

(75) Ardhanareeswaran, K. and Mirotsou, M. (2013) Lung stem and progenitor cells. Respiration, 85 (2), 89-95. 


\title{
6 \\ Chemicals and Stem Cells in the Promotion of Regeneration
}

\author{
Dikshya Bastakoty ${ }^{1}$, Sarika Saraswati ${ }^{2}$, and Pampee P. Young ${ }^{3}$ \\ ${ }^{I}$ Department of Pathology, Vanderbilt University, USA \\ ${ }^{2}$ Department of Pathology, Microbiology and Immunology, Vanderbilt University School of \\ Medicine, USA \\ ${ }^{3}$ Department of Pathology, Microbiology and Immunology, Vanderbilt University, USA
}

\subsection{Introduction}

Living beings possess an inherent ability to repair normal wear-and-tear of tissue caused by daily activities; this attribute is known as physiological regeneration [1]. Examples of physiological regeneration include replacement of blood cells, turnover of epithelial cells of the gut and skin, and renewal of endometrium after menstrual cycle. On the other hand, reparative regeneration refers to the replenishment of cells or tissues after post-traumatic injury. Large cellular or tissue loss due to trauma leads to the activation of specific signaling pathways aimed at repairing injury, which in some cases completely heals the injury and preserves the functionality of the lost tissue and in others leaves a healed but nonfunctional (fibrotic) scar tissue. The former type of healing occurs in many vertebrates, such as zebrafish, while the latter is more common in mammals. Through a better understanding of the process of regeneration in these diverse species, the field of regenerative medicine works toward developing ways of maximizing repair and minimizing fibrosis or scarring in humans.

As the world's aging population grows, there is an increasing demand on medicine to slow down or reverse the degeneration of organs and tissues. The US National Institutes of Health

Chemical Biology in Regenerative Medicine: Bridging Stem Cells and Future Therapies, First Edition. Edited by Charles C. Hong, Ada S. Ao, and Jijun Hao.

(C) 2014 John Wiley \& Sons, Ltd. Published 2014 by John Wiley \& Sons, Ltd. 
$(\mathrm{NIH})$ reported in 2010 that the world market for replacement organ therapies is $\$ 350$ billion, and that for regenerative medicine it is $\$ 500$ billion [2]. As a result of this increasing demand, the field of regenerative medicine, which started with surgical implants and transplants in the 1950s, has grown to encompass cell therapy, novel chemicals and biologics, and increasingly sophisticated biomaterials for scaffold or delayed delivery of chemicals.

With the discovery of stem cells and their potential to replace various cells in the adult organisms, stem-cell therapy has become the mainstay of modern regenerative medicine. The potential of stem cells to regenerate lost tissue depends on several parameters, including their delivery into the injured tissue, their differentiation into the desired cell type, their cooperative integration in the injury site to form functional tissue and - most important of all - their survival in the injured microenvironment. Hence the intrinsic healing potential of stem cells is better harnessed with the use of growth factors, biologics, small molecules, and biomaterials that aid their delivery, survival, and integration into the wound. In this review we will outline the use of these approaches to promote regeneration both in conjunction with stem cells and independent of cell therapy. We will particularly focus on the use of small molecules in stem-cell therapy in modern regenerative medicine.

\subsection{Biologics in Regenerative Medicine}

Biologics, in the widest sense, are agents derived from proteins produced by organisms. Growth factors, antibodies, and vaccines are some of the most common biologics that have been used to treat or prevent illnesses of various forms. In regenerative medicine, growth factors are used extensively and successfully for a number of indications $[3,4]$. With increased understanding of the signaling pathways implicated in tissue regeneration, antibodies or proteins that antagonize or activate certain pathways have emerged as therapeutic candidates. Some common biologics-based approaches in regenerative medicine are discussed in this section.

\subsubsection{Growth Factors and Pro-Angiogenic Agents}

Ever since their Nobel Prize-winning discovery by Rita Levi-Montalcini and Stanley Cohen in the 1950s [5,6], growth factors have been reported to play increasingly diverse and important roles in cell growth and differentiation. These roles lend them importance not only in development but also in regeneration after injury [3] (clinicaltrials.org: NCT00000842). Epidermal growth factor (EGF) is found to enhance healing of wounds in the skin and cornea [7]. Nerve growth factor (NGF) and brain-derived or glial cell-derived neurotropic factors (BDNF and GDNF, respectively) have been shown in rat models of nerve damage to enhance neuronal regeneration [8,9]. Similarly, platelet-rich plasma (PRP) has been shown in numerous animal studies to enhance tissue regeneration after injuries [10]. The plasma extracted from blood is enriched in the laboratory for platelets, which contain presynthesized growth factors packaged in $\alpha$-granules. Upon activation of clotting (after infusion into the injured tissue) these growth factors are secreted into the tissue microenvironment. Autologous transplant of PRP for the treatment of various degenerative diseases of tissue or bone has reached clinical trials (clinicaltrials.gov: NCT00761423, NCT01355549). Although the results from many of the trials and existing clinical reports have been 
promising (but inconsistent or ambiguous at best [11,12]), there still seems to be anticipation that with proper standardization of the techniques for plasma isolation, enrichment, storage, and delivery, better and more consistent outcomes can be expected. Pro-angiogenic factors such as vascular-endothelial growth factor (VEGF), fibroblast growth factor (FGF), and platelet-derived growth factor (PDGF) are another subclass of growth factors that have been utilized to improve regeneration by inducing neovascularization at the site of injury $[13,14]$.

\subsubsection{Immune-Modulatory Therapies}

The immune system plays an important role in the resolution of injury. Early after injury, it clears the site of injury of debris and infectious agents, and allows repair to begin. However, persistent and uncontrolled inflammation can be detrimental to wound repair [15], so drugs that modulate the inflammatory response have long been utilized to enhance it. Thrombin peptide (THP508) was reported to enhance fracture healing through activation of tumor necrosis factor- $\alpha(\mathrm{TNF} \alpha)$ and other pro-inflammatory factors [16]. On the other hand, attenuation of inflammatory response has also been shown to enhance regenerative repair. Several reports have shown that administration of high doses of nonsteroidal antiinflammatory drugs improve regeneration of brain tissue after the ischemic injury induced by a stroke in rodents $[17,18]$. Even the ability of stem-cell therapy using mesenchymal stromal or stem cells (MSCs) to enhance tissue regeneration is attributed in part to their immune-modulatory properties [19].

\subsubsection{Extracellular Matrix-Based Approaches}

The extracellular matrix (ECM) plays a key role in each step of injury repair. Within days after injury, a fibrous matrix is synthesized to replace lost or damaged tissue. The matrix is remodeled over time as the repair progresses. All the steps, from inflammation to reepithelialization to contraction, involve very close interactions with matricellular proteins, which sequester growth factors, regulate collagen turnover, and provide scaffold and support to allow cells to grow during tissue repair [20]. ECM components, such as heparin, bind many signaling molecules and growth factors and enhance their signaling by sequestering them to the microenvironment or by stabilizing them. They have been used to enhance signaling by proteins of interest [21]. Moreover, a polymer mimetic of heparan sulfate proteoglycan itself has demonstrated remarkable healing properties in both topical wounds and internal injuries [22,23]. A clinically available formulation is already in use in Europe and the Middle East to treat corneal ulcers and diabetic wounds [24]. Since heparan sulfates are important for the signaling of growth factors and cytokines, their degradation and cleavage in a wound microenvironment impairs healing. The heparan sulfate mimetics are uncleavable by heparanases and glycanases and hence aid repair of the wounded tissue [25].

\subsection{Chemicals and Biomaterials for Healing}

Biologics such as proteins and growth factors have the potential to significantly improve regeneration. However, in order for the healing agent to be effective it is important to ensure that it is delivered to the tissue of interest at a substantial concentration and that 
it is bioavailable at the site of injury. Hence, modern regenerative medicine is integrating diverse disciplines such as chemistry, material physics, and bioengineering in order to devise strategies for optimizing regeneration. Small molecules, bioactive scaffolds, and drug-delivery tools are the major new outcomes of these multidisciplinary investigations.

\subsubsection{Small Molecules}

A small molecule is a low-molecular-weight (smaller than 500 Daltons) organic compound. Small molecules that are investigated for use as therapeutics typically bind to a protein, nucleic acid, or polysaccharide and alter its enzymatic activity or biological function [26]. Small molecules are identified by cell phenotypic, reporter-based, or organism-based screening of chemical libraries [27]. Phenotypic screening is based on the cellular phenotype or cell-surface markers in response to the chemical library. In order to identify the modulators of specific pathways, promoter-driven reporters are used as readouts for the pathways of interest. Zebrafish and xenopus are routinely used for organism-based screens [28]. There is increasing interest in finding small molecules that can modulate signaling pathways as they have a number of advantages over biologics such as proteins and antibodies. For one thing, they are cheaper to synthesize. Due to their small size, they are often orally bioavailable, and hence delivery may not require injection. Additionally, they are more likely to be cell-permeable and thus to allow modification of the pathway at multiple steps; an antibody would rely solely on the sequesterization of a ligand or the blocking of the receptor. Hence, small molecules are very useful in blocking intracellular proteinprotein interactions, which has to date been the sole domain of antisense oligos. Small molecules thus offer a safer, more effective, and easier alternative to traditional signaling pathway-modulation approaches [26].

Given their superiority in modifying molecular signals, many types of small molecule have been investigated for potential roles in regeneration. Small-molecule antioxidants such as quercetin, allopurinol retinoids, and uric acid are reported to enhance wound healing [29]. Ascorbic acid, another small molecule, commonly used for stem-cell differentiation, is also known to regulate ECM by increasing collagen synthesis [30]. In a model of combined injury (fracture/irradiation) in mice, Greenberger and colleagues showed that the small molecule GS-Nitroxide attenuates ionizing radiation-driven delay in fracture healing [31]. Another small molecule, Pirfenidone, has been evaluated in clinical trials as an antifibrotic agent for idiopathic pulmonary fibrosis. Already reported to reduce fibrosis in lung, liver, heart, and kidney through the modulation of the transforming growth factor beta (TGF $\beta$ ) pathway, the molecule has potential for therapeutic application in tissue injury, through promoting regeneration by minimizing scarring [32]. Work by our group has also shown that in a mouse myocardial infarct model, treatment with the small-molecule Wnt inhibitor Pyrvinium alleviates adverse cardiac remodeling post-infarct [33]. The same molecule, upon topical treatment on mouse-ear punch injury, caused dramatic closure of the holes with remarkable restoration of tissue structure, including hair-follicle regeneration [34]. However, careful and thorough screenings of these molecules need to be performed before they can be proposed for therapeutic purposes, since some may have toxicity and off-target effects. Nonetheless, with careful considerations of their shortcomings, the exciting prospects for small molecules in advancing regenerative medicine cannot be overlooked. 


\subsubsection{Biomaterial Scaffold and Sustained Delivery}

As the field of regenerative medicine has become more multidisciplinary, innovative approaches to drug delivery, in vitro organ regeneration, and cell therapy have been introduced. These new technologies have revolutionized the field of regenerative medicine by increasing the efficacy and versatility of the traditional methods of enhancing regeneration through cell- or drug-based approaches.

Tissue engineering has been an important part of the field of regenerative medicine for many decades now. With the design and application of sophisticated biomaterials, tissue engineering is moving even further toward improving regenerative therapy [35]. Degradable biomaterials composed of collagen, fibronectin, chitosan, and so on have been used for the delivery of cells and to provide ECM components for the survival or directed differentiation of injected cells. Alginate, an algae-derived polysaccharide, has been used in the form of sponge or gel to enhance the regeneration of peripheral nerve [36], spinal cord [37], or excised axons [38] in rodent or feline models when used alone or in combination with neural progenitor (neurosphere) cells [39]. In a remarkable study, Atala and colleagues used a cell-polymer matrix composed of a Poly (Lactide-co-Glycolide)-poly(D,L-lactideco-glycolide) (PGA-PLGA) scaffold lined with urothelial cells on one side and muscle cells on the other to show for the first time the reconstitution of an autonomous and functional hollow organ using a tissue-engineering approach [40].

Another avenue in which bionics and biomaterial science are contributing to better tissue regeneration is in the sustained delivery of stabilized growth factors or drugs. In 2003, Hyongbum Kim et al. reported successfully using PLGA scaffolds for the slow release over a month of ascorbate-2-phosphate (AsAP) and dexamethasone to induce osteogenesis from MSCs [41]. Another recent study showed that an injectable polyvalent coacervate of a polycation and heparin used as a matrix for the sustained delivery and stabilization of FGF2 increased FGF2-mediated angiogenesis in a rodent model [21].

\subsection{Stem-Cell Therapy}

Stem cells were discovered by Becker et al. when they noted that bone-marrow cells injected into irradiated mice developed clonal nodules [42]. Subsequent studies by multiple groups have shown that stem cells are, by definition, self-renewing, multipotent cells. They exist in the embryo and in specific microenvironments called stem cell niches of tissues of the adult organism [43]. Although stem cells from different sources may vary in their degree of multipotency, virtually all stem cells (or progenitor cells, which are stem cells partially committed to a specific lineage) possess some degree of multipotency and capacity for self-renewal. These fundamental properties have placed stem cells on the forefront of regenerative biology. Also, there is increasing evidence to support the hypothesis that in almost all of the regenerative approaches mentioned in the preceding sections, true regeneration is driven by stem or progenitor cells in the wound milieu [44]. Although this statement may be partly contested, stem cells have for the past 2 decades been the buzzword in the context of regenerative therapies. By the year 2000, multiple studies had shown that stem cells from various tissues could give rise to unrelated cell types under special circumstances [45]. For example, Clarke et al. observed that neural stem cells 
(NSCs) transferred to a blastocyst could contribute to tissues belonging to all three germ layers [46]. These observations pointed toward their value in the regeneration of tissues that normally do not regenerate [45]. Ethical controversies surrounding embryonic stem cells (ESCs) stalled progress in stem-cell research, but the identification of stem cells in tissues of adult organisms opened up new avenues for research. Stem cells from various organs have now been used for many diseases and multiple types of injury, from bone injury, to degenerative diseases such as Alzheimer's, Parkinson's, and Huntington's, to cardiac, renal, and lung injuries [47]. In 2003, Lin et al. reported that hematopoietic stem cells (HSCs) transplanted into kidney after ischemia/reperfusion injury contributed to the regeneration of renal tubules [48]. MSCs from the bone marrow have perhaps been used most extensively in regenerative medicine for their ease of isolation and characterization, their multipotency, and most importantly their immunosuppressive properties. The latter have made allogeneic transplantation possible, increasing the feasibility of MSC transplant therapy [49]. Multiple studies using diverse injury models have reported improved regeneration by stem-cell transplantation [50,51]. This has led to several clinical trials aimed at improving regeneration using stem-cell therapy (clinicaltrials.gov: NCT01763099, NCT00877903). Not all of these studies have unambiguously shown remarkable success. However, with improved mechanistic insight and technical standardization, there is potential for improvement. Moreover, progress in tissue engineering and chemical biology is now providing excellent tools by which we can better understand stem cell biology and enhance the efficacy of stem-cell therapy.

\subsubsection{Chemical Manipulation of Stem Cells in Regeneration}

In order to be useful for clinical purposes, stem cells should maintain their self-renewal ability and be able to differentiate into specific lineages. Several biologics and genetic manipulations have been used to control the fate of stem cells, driving their self-renewal, differentiation, survival, or reprogramming. However, modulation of these complex biological events with small molecules seems to be safer and more effective in regulating stem-cell fate. Using high-throughput screening of chemical libraries, many molecules have been identified that target specific cellular pathways to maintain stem-cell self-renewal, direct stem-cell differentiation, and enable or enhance stem-cell reprogramming [27, 52]. Here we will focus on recent interventions introduced by small molecules in affecting and/or improving the behavior of different types of stem cells commonly used in regenerative biology.

\subsubsection{Embryonic Stem Cells (ESCs)}

\subsubsection{Small Molecules for the Culture and Maintenance of ESCs}

ESCs are stem cells derived from the blastocyst stage of the embryo $[53,54]$. These cells are pluripotent, clonal, and self-renewing, and can differentiate into the cells of three germ layers. They are therefore a strong candidate for regenerative therapies [55]. However, the use of ESCs is limited due to ethical considerations, potential rejection problems following their allogeneic transplantation, and the formation of teratomas after in vivo injection. Traditionally, ESCs require feeder cells (growth-inactivated fibroblasts), fetal bovine serum, growth factors, and knockout serum replacement in order to grow in culture. 
However, there is a lack of fully defined and reproducible culture conditions that would allow them to grow in vitro. Additionally, the need for interventions such as feeder cells and other animal serum products imposes risk of contamination. To overcome this issue, Chen et al. performed a high-throughput cell-based screening on a mouse ESC line. This led to identification of a small molecule (Pluripotin) that could maintain the self-renewal status of ESCs under serum-free, feeder cell-free, and growth factor (leukemia inhibitory factor, LIF)-free conditions [52]. Pluripotin exerts its effect by inhibiting Ras GTPaseactivating protein (RasGAP) and extracellular signal-regulated kinase-1 (ERK1). This was a breakthrough in the area of ESC research, since it was thought to be impossible to culture ESCs without feeder cells or any extrinsic growth factors. Following this study, another group used a combinatorial approach in which a mitogen-activated protein kinase kinase (MEK) inhibitor, PD0325901, and a glycogen synthase kinase 3 (GSK3) inhibitor, CHIR99021, were used to maintain mESC culture under cytokine-free and feeder-free conditions [56]. One persistent hurdle in the expansion of ESCs was the induction of cell death upon single-cell dissociation; this has been overcome by using the Rho-associated coiled coil-containing protein kinase (ROCK) inhibitor Y-27632 [57].

\subsubsection{Small Molecules for ESC Differentiation}

Besides improving ESCs' culture conditions, small molecules have also been successfully utilized to drive differentiation of ESCs into different cell lineages. In order to allow them to be utilized for regenerative and other clinical applications, culture conditions have been developed that drive ESCs toward hematopoietic cells [58], pancreatic cells [59], endothelial cells [60], and other cell types [61]. However, the poor efficiency of differentiation and the lack of homogeneity in the differentiated cell population are among the major challenges still facing this endeavor. Small molecules may also provide more specific and vigorous differentiation while allowing mechanistic insight into the lineage differentiation process. The PI3Kinase inhibitor LY294002 has been shown to prime human ESCs (hESCs) toward the endoderm differentiation induced by activin-A [62]. Another chemical, stauprimide, targeted nonmetastatic cell 2 (NME2) and cMyc to prime ESCs toward endoderm lineage specification [63]. In an hESC differentiation screen, SB431542, a TGF $\beta$ receptor-I inhibitor, directed ESCs toward endothelial lineage commitment. Dualinhibition strategies have been used to obtain more precise and focused outcomes. For example, the inhibition of bone morphogenetic protein 4 (BMP4) by Noggin and that of TGF $\beta$ by SB431542 leads to more directed and efficient (larger number of differentiated cells obtained in shorter time) neural differentiation of hESCs. The idea behind this dualinhibition strategy was to drive ESCs toward neural differentiation while inhibiting ESC commitment toward other fates. A high-content screen (an approach that allows the study of several biological and morphological changes simultaneously at the single-cell level) of a chemical library by Chen et al. identified a small molecule, (-)-indolactam V (ILV), which through protein kinase $\mathrm{C}$ signaling dramatically $(>45 \%)$ enhances the number of Pdx1-expressing pancreatic progenitor cells derived from hESCs [64]. These cells express other pancreatic markers and participate in the generation of endocrine, exocrine, and duct cells, in vitro and in vivo. Efficient generation of specific cell types from ESCs could be very helpful in disease conditions such as Parkinson's and juvenile-onset diabetes mellitus that are caused by the death or abnormality of a few specific cell types [55]. Chemical 
screening for in vitro differentiation of ESCs toward specific lineages is a promising tool by which to identify targets for the development of drugs for use in regenerative therapies.

\subsubsection{Induced Pluripotent Stem Cells (iPSCs)}

\subsubsection{Generation of iPSCs}

Although ESCs have great potential in regenerative medicine, ethical considerations, the potential to form teratomas, and the possibility of rejection following transplantation limit their utilization for therapeutic purposes. Generation of induced pluripotent stem cells (iPSCs) from the patient's own somatic cells seems to bypass these problems. iPSCs are pluripotent stem cells that are generated from an adult somatic cell via the forced expression of transcription programs affecting stemcellness. Nobel Prize-winning work by Dr Yamanaka's group has shown that virus-mediated overexpression of Oct4, Sox2, Klf4, and $M y c$ can induce the conversion of mouse fibroblasts into iPSCs [65]. The gene expression patterns, epigenetic changes, and developmental potentials of these iPSCs closely matched those of the mouse ESCs (mESCs). In another study, the same group was able to reprogram human fibroblasts toward iPSCs using a similar strategy [66]. Although this is a very promising approach in regenerative medicine, the poor efficiency of iPSC generation and the use of a retrovirus for the delivery of iPSC-generating factors remain among the major hurdles. Virus integration can affect the quality of iPSCs and may cause tumorigenicity in the resulting iPSCs. Fortunately, recent alternatives for the delivery of reprogramming factors, such as reprogramming mRNA [67], proteins [68], microRNAs [69], excisable expression systems [70], and episomal plasmids [71], have provided alternatives by which to resolve the problems associated with virus-mediated delivery systems.

\subsubsection{Small Molecules that Affect iPSC Epigenomes}

Despite these advances there is still a need for more efficient ways of reprogramming iPSCs. Phenotypic screening of chemicals has been useful in identifying small molecules that can functionally replace the transcription factors required for iPSC reprogramming and enhance the efficiency of reprogramming. An inhibitor of histone methyl transferase, BIX-01294, was found to significantly enhance the Oct4-Klf4 mediated reprogramming of neural progenitor cells [72] and of mouse embryonic fibroblasts into iPSCs in two different studies [73]. Valproic acid and Trichostatin A, which are inhibitors of another histonemodifying enzyme, histone deacetylase, have also improved the reprogramming efficiency $[74,75]$. Valproic acid in particular is widely used to enhance the reprogramming of human fibroblasts [76] and mouse embryonic fibroblasts [68].

\subsubsection{Small Molecules that Affect iPSC Signaling Pathways}

During the reprogramming process, dramatic changes occur in gene expression and cell signaling due to a fundamental process known as mesenchymal-epithelial transition (MET). Small molecules specifically targeting this process have been very useful in reprogramming. Blocking of TGF $\beta$ signaling by the TGF $\beta$-receptor inhibitor SB431542 and of a downstream target MEK by its inhibitor PD0325901 both enhance MET. Simultaneously, these molecules significantly enhance reprogramming efficiency [77]. The Wnt signaling pathway has been shown to enhance reprogramming by acting on T-cell factor-3 [78]. 
A GSK3 inhibitor, CHIR99021, facilitated reprogramming of mouse embryonic fibroblasts into iPSCs by activating Wnt signaling. CHIR99021 and the lysine-specific demethylase 1 (LSD1) inhibitor Parnate enabled the reprogramming of human primary keratinocytes in the presence of Oct4-Klf4 [79]. As a positive further step, Dr Zhu's group developed a cocktail of chemicals consisting of A-83-01 (an inhibitor of the TGF $\beta 1$ receptors ALK4, ALK5, and ALK7), sodium butyrate (a histone deacetylase (HDAC) inhibitor), and PD0325901 and PS48 ( $3^{\prime}$-phosphoinositide-dependent kinase-1 activators) that facilitated the generation of human iPSCs from multiple human somatic cell lines [80]. However, in addition to the cocktail, the exogenous expression of Oct4 was necessary. Another interesting factor which contributes to reprogramming and has been targeted by small molecules is cellular metabolism. PS48, as found in this chemical cocktail, enabled a metabolic switch from mitochondrial oxidative phosphorylation toward glycolysis during the reprogramming process [80]. This switch in energy metabolism by pluripotent stem cells would favor pluripotency by stimulating proliferation with reduced generation of reactive oxygen species.

\subsubsection{Mesenchymal Stem Cells (MSCs)}

\subsubsection{Properties of MSCs}

MSCs are multipotent stem cells that have the ability to self-renew and the potential to differentiate into multiple lineages, including adipogenic, chondrogenic, and osteogenic. MSCs were first identified by Dr Friedenstein as colony-forming-unit fibroblasts (CFU-Fs) [81] isolated from the bone marrow. Other than bone marrow, MSCs can also be isolated from adipose tissue [82], blood [83], dermis [84], and muscle [85]. MSCs possess plastic adherent properties and are clonogenic. Initially, they were used as feeder monolayers for the HSC culture. MSCs have been implicated in promoting regeneration and this is largely attributed to their immune-modulating properties and their secretome [86]. MSCs home to the injury site to aid in the tissue-repair process [87]. The use of MSC-based therapies is receiving significant consideration because they are easy to isolate, can be readily expanded, and are considered immunosuppressive due to the lack of Human Leukocyte Antigen (HLA) class II antigens. Hence, MSCs have been used in various indications either alone or in combination with genetic manipulation, biomaterial scaffolds [49], growth factors, or small molecules [88].

\subsubsection{Small Molecules that affect MSC Differentiation}

Although MSCs have an inherent ability to differentiate into multiple lineages in vitro, this differentiation is aided by specific factors [89]. A synthetic agonist, purmorphamine, promotes osteogenic differentiation of MSCs by activating hedgehog or BMP signaling $[90,91]$. A PPAR- $\gamma$ activating drug, rosiglitazone, induces the generation of adipocytes from both MSCs and ESCs [92]. Since MSCs have multilineage differentiation potential, they are a very strong candidate for cartilage repair; specifically, for the treatment of degenerative diseases such as osteoarthritis (OA). According to the Centers for Disease Control and Prevention (CDC), there are currently around 27 million people suffering from OA in the United States alone [93]. An MSC-like population has been identified living in adult cartilage that has the potential to differentiate into chondrocytes and repair the damaged cartilage [94]. The identification of small molecules capable of enhancing the regenerating potential of these endogenous stem-cell populations for cartilage repair, when delivered 
locally, could be of tremendous help. Image-based screening of chemical libraries has identified a small molecule, kartogenin (KGN), that can enhance the formation of chondrogenic nodules in primary human MSCs [95]. The mechanism of KGN induction of chondrogenesis has been suggested to lie in its regulation of the RUNX family of transcription factors, which are key players in MSC-mediated chondrogenesis. Finding ways of controlling MSC fate using small molecules will have a major impact in the area of tissue engineering and regeneration. High-throughput screenings have been very useful in the identification of more potent small-molecule candidates that can enhance the osteogenic capability of MSCs $[96,97]$. Work by Park et al. has identified that an amiloride derivative, phenamil, stimulates osteoblast differentiation and mineralization in MSCs by acting on the BMP signaling pathway [98]. Another small molecule, 5-aminoimidazole-4-carboxamide-1- $\beta$ riboside (AICAR), which activates AMP-activated protein kinase (AMPK), significantly inhibits human and rabbit MSC proliferation and competently promotes the differentiation of human and rabbit MSCs toward osteogenic lineage [99].

A major hurdle in MSC-mediated regenerative therapy is the poor survival of cells after transplantation in vivo. A study performed by Chen et al. identified that cholesterol myristate suppresses apoptosis of MSCs by activating inhibitor of differentiation (ID1), a downstream target of BMP4 signaling [100]. Furthermore, our group identified that a small-molecule Wnt-signaling inhibitor, Pyrvinium, enhanced MSC proliferation in vitro and MSC engraftment in vivo while suppressing the differentiation potential of MSCs [101]. These studies are promising and are of great help in developing strategies for exploiting small molecules in the direction of enhancing and exploiting the function of MSCs for regenerative purposes.

\subsubsection{Biopolymers that affect MSC Biology}

The generation of a scaffold with small-molecule chemical functional groups for MSC delivery is emerging as a novel approach for MSC-based therapies. The functional 3D culture environment can mimic the in vivo microenvironment of a stem cell niche. The significance of using biopolymers (consisting of small molecules) for drug delivery has already been discussed earlier in this chapter. In a pilot study by Radice et al., a hyaluronanbased scaffold was identified as a safe and efficient vehicle for MSC delivery in articular cartilage defects [102]. In another study, hydrogels were made with tethered functional small molecules and human MSCs were encased and cultured in this scaffold [103]. Interestingly, small chemicals attached to the hydrogel induced MSCs to differentiate into osteogenic and adipogenic lineages. Although these studies are promising, much work is needed to develop strategies that can identify more targeted and efficient small molecules by which to enhance MSC-mediated regeneration. Utilizing simple chemistry to manipulate complex biological phenomena is an exciting way of improving MSC function for therapeutic utilization.

\subsubsection{Hematopoietic Stem Cells (HSCs)}

HSCs are bone marrow-derived progenitor cells that can regenerate all the mature blood cells and have been implicated in the regeneration of other tissues [104]. These cells can also be isolated from the placenta. Because they are immunologically naïve, they are great options for allogeneic transplantation. However, only a small number of HSCs are present 
in the bone marrow and placenta. Therefore, strategies are being developed to improve their isolation and expansion for bone-marrow transplantation and other purposes. For example, HSC growth is very sensitive to extracellular copper concentrations and the copper chelator tetraethylenepentamine increases HSC proliferation and enhances their engraftment in mice [105]. Tetraethylenepentamine has been found to be safe in phase I/II clinical trial when added to umbilical-cord blood (UCB) [106]. Chemical screening has identified a small molecule, StemReginin 1, that increases HSC number and engraftment in mice by targeting the aryl hydrocarbon receptor, the role of which is not very well understood [107]. UCB is widely used as an alternative source of HSC transplantation for patients lacking proper HLA-matched adult donors [104]. Although this is a valuable source of stem cells, the relatively small number poses a significant problem of delayed engraftment. A number of strategies have been developed to expand progenitors in UCB in order to reduce time to engraftment. Specifically, Notch signaling and hematopoietic growth factors have been implicated in enhancing the proliferation of HSCs [108]. A significant increase in human and mouse HSC number was detected when Notch ligands, Delta, and Jagged treatments were employed $[109,110]$. The safety and efficacy of Notch ligands were determined by their successful utilization in a phase I clinical trial for myeloablative UCB transplantation [109]. In addition, prostaglandin E2 has also been implicated in HSC proliferation [111]. Live animal chemical screening identified compounds that activate prostaglandin E2 and enhance HSC proliferation.

\subsection{Conclusion}

Regenerative biology has grown from its humble beginnings more than half a century ago into a rapidly expanding, multidisciplinary field of research with immense clinical impact. With the discovery of stem cells, and of their potential to accelerate tissue regeneration, stem-cell therapy has become almost synonymous with regenerative medicine. Furthermore, the integration of chemical biology, material science, and biomedical engineering with stem-cell biology has pushed back the frontiers of the field. Novel small molecules that allow the chemical modification of stem cells or modulate signaling pathways implicated in tissue regeneration are increasingly being synthesized and used for regeneration. Moreover, use of scaffolds, ECM-mimetics, nanoparticles, and the like is making delivery of the aforementioned stem cells and small molecules increasingly effective. A summary of the effects on wound repair and regeneration of biologics, small molecules, and biomaterials both alone and in conjunction with stem cells is given in Figure 6.1.

Regenerative biology is leading all other fields of biomedical research in setting new records for rapid translation into the clinic. Such rapid transition inevitably carries risks of limited efficacy or limited safety; encouragingly, however, safety issues have been minimal so far. In regards to efficacy, ongoing efforts to standardize and regulate the techniques used for the isolation, expansion, and storage of stem cells, as well as more effective chemicals and small molecules, are likely to considerably improve the outlook of this therapeutic approach in the clinic.

Stem cells have the potential to heal injury when injected/transplanted alone in the wound microenvironment. Biologics (proteins, proteoglycans, and other biological factors), small molecules, and biomaterials (scaffolds, ECM-mimetics, and nanoparticles) can also aid the 


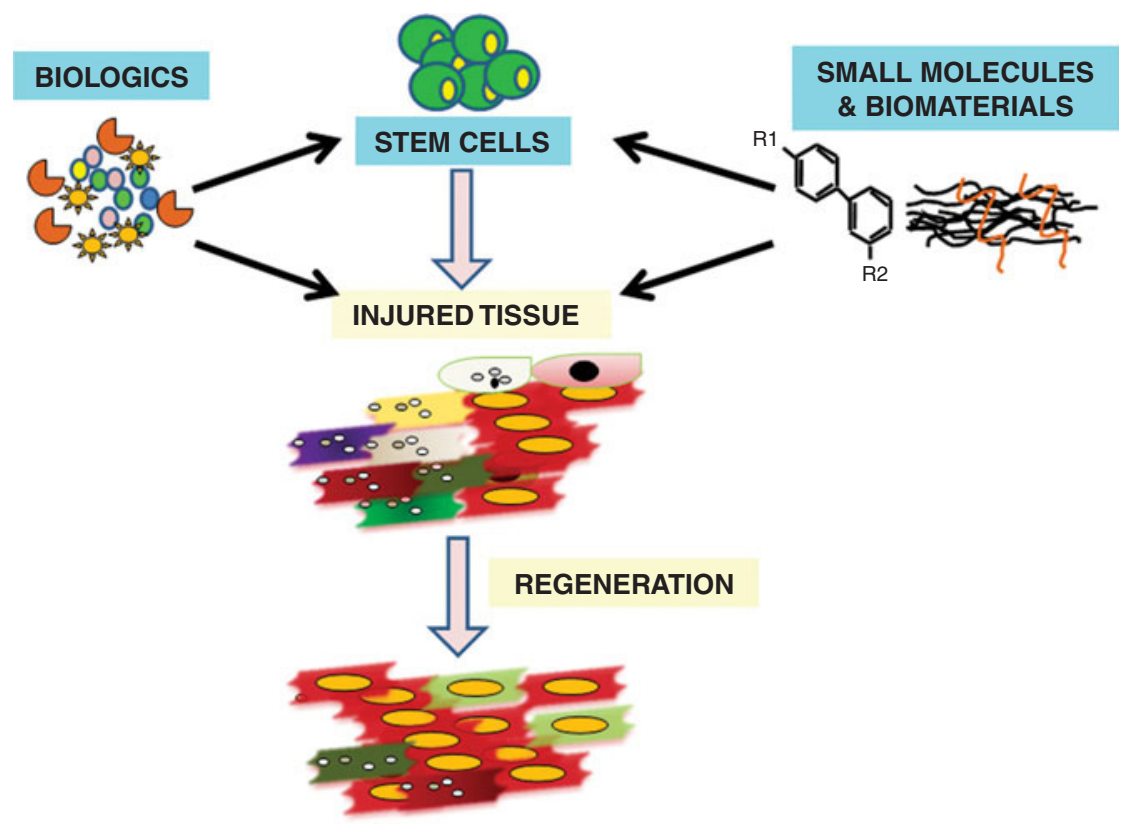

Figure 6.1 Stem cells, biologics, and chemicals aid injury repair

wound-repair process by directly affecting the injured microenvironment or enhancing the wound-healing capabilities of stem cells.

\section{References}

(1) Carlson, B.M. (2007) An Introduction to Regeneration Principles of Regenerative Biology, 1st edn, Academic Press, London, UK, pp. 1-30.

(2) Stocum, D.L. (2002) Regenerative biology and medicine. Journal of Musculoskeletal \& Neuronal Interactions, 2 (3), 270-273.

(3) De Boer, J., Wang, H.J., and Van Blitterswijk, C. (2004) Effects of Wnt signaling on proliferation and differentiation of human mesenchymal stem cells. Tissue Engineering, 10 (3-4), 393-401.

(4) Baek, S.H., Kioussi, C., Briata, P. et al. (2003) Regulated subset of G1 growth-control genes in response to derepression by the Wnt pathway. Proceedings of the National Academy of Sciences of the United States of America, 100 (6), 3245-3250.

(5) Cho, H.H., Kim, Y.J., Kim, S.J. et al. (2006) Endogenous Wnt signaling promotes proliferation and suppresses osteogenic differentiation in human adipose derived stromal cells. Tissue Engineering, 12 (1), 111-121.

(6) Cohen, S., Levi-Montalcini, R., and Hamburger, V. (1954) A nerve growth-stimulating factor isolated from Sarcom as 37 and 180. Proceedings of the National Academy of Sciences of the United States of America, 40 (10), 1014-1018.

(7) Zhang, D.Y., Wang, H.J., and Tan, Y.Z. (2011) Wnt/beta-catenin signaling induces the aging of mesenchymal stem cells through the DNA damage response and the p53/p21 pathway. PLoS One, 6 (6), e21397. 
(8) Kokai, L.E., Bourbeau, D., Weber, D. et al. (2011) Sustained growth factor delivery promotes axonal regeneration in long gap peripheral nerve repair. Tissue Engineering Part A, 17 (9-10), $1263-1275$.

(9) Lindsay, R.M. (1988) Nerve growth factors (NGF, BDNF) enhance axonal regeneration but are not required for survival of adult sensory neurons. The Journal of Neuroscience, $\mathbf{8}$ (7), 2394-2405.

(10) Marx, R.E. (2004) Platelet-rich plasma: evidence to support its use. Journal of Oral and Maxillofacial Surgery, 62 (4), 489-496.

(11) Foster, T.E., Puskas, B.L., Mandelbaum, B.R. et al. (2009) Platelet-rich plasma: from basic science to clinical applications. American Journal of Sports Medicine, 37 (11), 2259-2272.

(12) Pradeep, A.R., Pai, S., Garg, G. et al. (2009) A randomized clinical trial of autologous platelet-rich plasma in the treatment of mandibular degree II furcation defects. Journal of Clinical Periodontology, 36 (7), 581-588.

(13) Frontini, M.J., Nong, Z., Gros, R. et al. (2011) Fibroblast growth factor 9 delivery during angiogenesis produces durable, vasoresponsive microvessels wrapped by smooth muscle cells. Nature Biotechnology, 29 (5), 421-427.

(14) Chu, H. and Wang, Y. (2012) Therapeutic angiogenesis: controlled delivery of angiogenic factors. Therapeutic Delivery, 3 (6), 693-714.

(15) Stroncek, J.D. and Reichert, W.M. (2008) Overview of wound healing in different tissue types environment, in Indwelling Neural Implants: Strategies for Contending with the In Vivo Environment (ed. W.M. Reichert), CRC Press, Boca Raton, FL, Chapter 1.

(16) Ryaby, J.T., Sheller, M.R., Levine, B.P. et al. (2006) Thrombin peptide TP508 stimulates cellular events leading to angiogenesis, revascularization, and repair of dermal and musculoskeletal tissues. Journal of Bone and Joint Surgery. American Volume, 88 (Suppl. 3), 132-139.

(17) Wang, J., Liu, Y.M., Cao, W. et al. (2012) Anti-inflammation and antioxidant effect of Cordymin, a peptide purified from the medicinal mushroom Cordyceps sinensis, in middle cerebral artery occlusion-induced focal cerebral ischemia in rats. Metabolic Brain Disease, 27 (2), 159-165.

(18) Bazan, N.G., Eady, T.N., Khoutorova, L. et al. (2012) Novel aspirin-triggered neuroprotectin D1 attenuates cerebral ischemic injury after experimental stroke. Experimental Neurology, 236 (1), 122-130.

(19) English, K. and Mahon, B.P. (2011) Allogeneic mesenchymal stem cells: agents of immune modulation. Journal of Cellular Biochemistry, 112 (8), 1963-1968.

(20) Midwood, K.S., Mao, Y., Hsia, H.C. et al. (2006) Modulation of cell-fibronectin matrix interactions during tissue repair. Journal of Investigative Dermatology Symposium Proceedings, 11 (1), 73-78.

(21) Chu, H., Gao, J., Chen, C.W. et al. (2011) Injectable fibroblast growth factor-2 coacervate for persistent angiogenesis. Proceedings of the National Academy of Sciences of the United States of America, 108 (33), 13444-13449.

(22) van Neck, J., Tuk, B., Barritault, D. and Tong, M. (2012) Heparan sulfate proteoglycan mimetics promote tissue regeneration: an overview, in Tissue Regeneration - From Basic Biology to Clinical Application (ed. J. Davies), InTech, Chapter 4.

(23) Barritault, D. and Caruelle, J.P. (2006) Regenerating agents (RGTAs): a new therapeutic approach. Annales Pharmaceutiques Francaises, 64 (2), 135-144.

(24) Xiao, Y.T., Xiang, L.X., and Shao, J.Z. (2007) Bone morphogenetic protein. Biochemical and Biophysical Research Communications, 362 (3), 550-553.

(25) Tong, M., Tuk, B., Hekking, I.M. et al. (2009) Stimulated neovascularization, inflammation resolution and collagen maturation in healing rat cutaneous wounds by a heparan sulfate glycosaminoglycan mimetic, OTR4120. Wound Repair and Regeneration, 17 (6), 840-852.

(26) Arkin, M.R. and Wells, J.A. (2004) Small-molecule inhibitors of protein-protein interactions: progressing towards the dream. Nature Reviews Drug Discovery, 3 (4), 301-317.

(27) Desbordes, S.C., Placantonakis, D.G., Ciro, A. et al. (2008) High-throughput screening assay for the identification of compounds regulating self-renewal and differentiation in human embryonic stem cells. Cell Stem Cell, 2 (6), 602-612. 
(28) Thorne, C.A., Hanson, A.J., Schneider, J. et al. (2010) Small-molecule inhibition of Wnt signaling through activation of casein kinase 1alpha. Nature Chemical Biology, 6 (11), 829836.

(29) Wattamwar, P.P. and Dziubla, T.D. (2012) Modulation of the wound healing response through oxidation active materials, in Engineering Biomaterials for Regenerative Medicine: Novel Technologies for Clinical Applications (ed. S.K. Bhatia), Springer, Chapter 1.

(30) Murad, S., Grove, D., Lindberg, K.A. et al. (1981) Regulation of collagen synthesis by ascorbic acid. Proceedings of the National Academy of Sciences of the United States of America, 78 (5), 2879-2882.

(31) Gokhale, A., Rwigema, J.C., Epperly, M.W. et al. (2010) Small molecule GS-nitroxide ameliorates ionizing irradiation-induced delay in bone wound healing in a novel murine model. In Vivo (Athens, Greece), 24 (4), 377-385.

(32) Schaefer, C.J., Ruhrmund, D.W., Pan, L. et al. (2011) Antifibrotic activities of pirfenidone in animal models. European Respiratory Review, 20 (120), 85-97.

(33) Saraswati, S., Alfaro, M.P., Thorne, C.A. et al. (2010) Pyrvinium, a potent small molecule Wnt inhibitor, promotes wound repair and post-MI cardiac remodeling. PLoS One, 5 (11), e15521.

(34) Kosinski, M. Improved wound healing through topical application of a small molecule. Available from: http://www.vanderbilt.edu/cttc/execsumm/MKT11100.pdf (last accessed March 10, 2014).

(35) Furth, M.E., Atala, A., and Van Dyke, M.E. (2007) Smart biomaterials design for tissue engineering and regenerative medicine. Biomaterials, 28 (34), 5068-5073.

(36) Suzuki, Y., Tanihara, M., Ohnishi, K. et al. (1999) Cat peripheral nerve regeneration across 50 $\mathrm{mm}$ gap repaired with a novel nerve guide composed of freeze-dried alginate gel. Neuroscience Letters, 259 (2), 75-78.

(37) Suzuki, K., Suzuki, Y., Ohnishi, K. et al. (1999) Regeneration of transected spinal cord in young adult rats using freeze-dried alginate gel. NeuroReport, 10 (14), 2891-2894.

(38) Kataoka, K., Suzuki, Y., Kitada, M. et al. (2001) Alginate, a bioresorbable material derived from brown seaweed, enhances elongation of amputated axons of spinal cord in infant rats. Journal of Biomedical Materials Research, 54 (3), 373-384.

(39) Wu, S., Suzuki, Y., Kitada, M. et al. (2001) Migration, integration, and differentiation of hippocampus-derived neurosphere cells after transplantation into injured rat spinal cord. $\mathrm{Neu}$ roscience Letters, 312 (3), 173-176.

(40) Oberpenning, F., Meng, J., Yoo, J.J., and Atala, A. (1999) De novo reconstitution of a functional mammalian urinary bladder by tissue engineering. Nature Biotechnology, 17 (2), 149-155.

(41) Kim, H., Kim, H.W., and Suh, H. (2003) Sustained release of ascorbate-2-phosphate and dexamethasone from porous PLGA scaffolds for bone tissue engineering using mesenchymal stem cells. Biomaterials, 24 (25), 4671-4679.

(42) Becker, A.J., Mc, C.E., and Till, J.E. (1963) Cytological demonstration of the clonal nature of spleen colonies derived from transplanted mouse marrow cells. Nature, 197, 452-454.

(43) Bajada, S., Mazakova, I., Richardson, J.B., and Ashammakhi, N. (2008) Updates on stem cells and their applications in regenerative medicine. Journal of Tissue Engineering and Regenerative Medicine, 2 (4), 169-183.

(44) Bongso, A. and Richards, M. (2004) History and perspective of stem cell research. Best Practice \& Research Clinical Obstetrics \& Gynaecology, 18 (6), 827-842.

(45) Andrews, P.W. (2002) From teratocarcinomas to embryonic stem cells. Philosophical Transactions of the Royal Society of London. Series B, Biological Sciences, 357 (1420), 405-417.

(46) Clarke, D.L., Johansson, C.B., Wilbertz, J. et al. (2000) Generalized potential of adult neural stem cells. Science, $\mathbf{2 8 8}$ (5471), 1660-1663.

(47) Ramakrishna, V., Janardhan, P.B. and Sudarsanareddy, L. (2011) Stem cells and regenerative medicine - a review. Annual Review \& Research in Biology. [Review], 1 (4), 30.

(48) Lin, F., Cordes, K., Li, L. et al. (2003) Hematopoietic stem cells contribute to the regeneration of renal tubules after renal ischemia-reperfusion injury in mice. Journal of the American Society of Nephrology, 14 (5), 1188-1199. 
(49) Eslaminejad, M.B., Zomorodian, E., and Bagheri, F. (2013) Mesenchymal stem cells in bone and cartilage regeneration, in Regenerative Medicine and Cell Therapy (eds H. Baharvand and N. Aghdami), Humana Press, pp. 131-153.

(50) Alfaro, M.P., Pagni, M., Vincent, A. et al. (2008) A Wnt modulator sFRP2 enhances mesenchymal stem cell engraftment, granulation tissue formation and myocardial repair. Proceedings of the National Academy of Sciences of the United States of America, 105, 18366-18371.

(51) Mirotsou, M., Zhang, Z., Deb, A. et al. (2007) Secreted frizzled related protein 2 (sFRP2) is the key Akt-mesenchymal stem cell-released paracrine factor mediating myocardial survival and repair. Proceedings of the National Academy of Sciences of the United States of America, 104, 1643-1648.

(52) Chen, S., Do, J.T., Zhang, Q. et al. (2006) Self-renewal of embryonic stem cells by a small molecule. Proceedings of the National Academy of Sciences of the United States of America, 103 (46), 17266-17271.

(53) Evans, M.J. and Kaufman, M.H. (1981) Establishment in culture of pluripotential cells from mouse embryos. Nature, 292 (5819), 154-156.

(54) Martin, G.R. (1981) Isolation of a pluripotent cell line from early mouse embryos cultured in medium conditioned by teratocarcinoma stem cells. Proceedings of the National Academy of Sciences of the United States of America, 78 (12), 7634-7638.

(55) Thomson, J.A., Itskovitz-Eldor, J., Shapiro, S.S. et al. (1998) Embryonic stem cell lines derived from human blastocysts. Science, 282 (5391), 1145-1147.

(56) Buehr, M., Meek, S., Blair, K. et al. (2008) Capture of authentic embryonic stem cells from rat blastocysts. Cell, 135 (7), 1287-1298.

(57) Watanabe, K., Ueno, M., Kamiya, D. et al. (2007) A ROCK inhibitor permits survival of dissociated human embryonic stem cells. Nature Biotechnology, 25 (6), 681-686.

(58) Feng, Q., Lu, S.J., Klimanskaya, I. et al. (2010) Hemangioblastic derivatives from human induced pluripotent stem cells exhibit limited expansion and early senescence. Stem Cells, $\mathbf{2 8}$ (4), 704-712.

(59) Kroon, E., Martinson, L.A., Kadoya, K. et al. (2008) Pancreatic endoderm derived from human embryonic stem cells generates glucose-responsive insulin-secreting cells in vivo. Nature Biotechnology, 26 (4), 443-452.

(60) James, D., Nam, H.S., Seandel, M. et al. (2010) Expansion and maintenance of human embryonic stem cell-derived endothelial cells by TGFbeta inhibition is Id1 dependent. Nature Biotechnology, 28 (2), 161-166.

(61) Chambers, S.M., Fasano, C.A., Papapetrou, E.P. et al. (2009) Highly efficient neural conversion of human ES and iPS cells by dual inhibition of SMAD signaling. Nature Biotechnology, 27 (3), 275-280.

(62) McLean, A.B., D'Amour, K.A., Jones, K.L. et al. (2007) Activin a efficiently specifies definitive endoderm from human embryonic stem cells only when phosphatidylinositol 3-kinase signaling is suppressed. Stem Cells, 25 (1), 29-38.

(63) Zhu, S., Wurdak, H., Wang, J. et al. (2009) A small molecule primes embryonic stem cells for differentiation. Cell Stem Cell, 4 (5), 416-426.

(64) Chen, S., Borowiak, M., Fox, J.L. et al. (2009) A small molecule that directs differentiation of human ESCs into the pancreatic lineage. Nature Chemical Biology, 5 (4), 258-265.

(65) Takahashi, K. and Yamanaka, S. (2006) Induction of pluripotent stem cells from mouse embryonic and adult fibroblast cultures by defined factors. Cell, 126 (4), 663-676.

(66) Takahashi, K., Tanabe, K., Ohnuki, M. et al. (2007) Induction of pluripotent stem cells from adult human fibroblasts by defined factors. Cell, 131 (5), 861-872.

(67) Warren, L., Manos, P.D., Ahfeldt, T. et al. (2010) Highly efficient reprogramming to pluripotency and directed differentiation of human cells with synthetic modified mRNA. Cell Stem Cell, 7 (5), 618-630.

(68) Zhou, H., Wu, S., Joo, J.Y. et al. (2009) Generation of induced pluripotent stem cells using recombinant proteins. Cell Stem Cell, 4 (5), 381-384.

(69) Anokye-Danso, F., Trivedi, C.M., Juhr, D. et al. (2011) Highly efficient miRNA-mediated reprogramming of mouse and human somatic cells to pluripotency. Cell Stem Cell, 8 (4), $376-388$. 
(70) Soldner, F., Hockemeyer, D., Beard, C. et al. (2009) Parkinson's disease patient-derived induced pluripotent stem cells free of viral reprogramming factors. Cell, 136 (5), 964-977.

(71) Yu, J., Hu, K., Smuga-Otto, K. et al. (2009) Human induced pluripotent stem cells free of vector and transgene sequences. Science, 324 (5928), 797-801.

(72) Shi, Y., Do, J.T., Desponts, C. et al. (2008) A combined chemical and genetic approach for the generation of induced pluripotent stem cells. Cell Stem Cell, 2 (6), 525-528.

(73) Shi, Y., Desponts, C., Do, J.T. et al. (2008) Induction of pluripotent stem cells from mouse embryonic fibroblasts by Oct 4 and Klf4 with small-molecule compounds. Cell Stem Cell, 3 (5), 568-574.

(74) Huangfu, D., Maehr, R., Guo, W. et al. (2008) Induction of pluripotent stem cells by defined factors is greatly improved by small-molecule compounds. Nature Biotechnology, 26 (7), 795-797.

(75) Mikkelsen, T.S., Hanna, J., Zhang, X. et al. (2008) Dissecting direct reprogramming through integrative genomic analysis. Nature, 454 (7200), 49-55.

(76) Huangfu, D., Osafune, K., Maehr, R. et al. (2008) Induction of pluripotent stem cells from primary human fibroblasts with only Oct4 and Sox2. Nature Biotechnology, 26 (11), 12691275.

(77) Lin, T., Ambasudhan, R., Yuan, X. et al. (2009) A chemical platform for improved induction of human iPSCs. Nature Methods, 6 (11), 805-808.

(78) Niwa, H. (2011) Wnt: what's needed to maintain pluripotency? Nature Cell Biology, 13 (9), $1024-1026$.

(79) Li, W., Zhou, H., Abujarour, R. et al. (2009) Generation of human-induced pluripotent stem cells in the absence of exogenous Sox2. Stem Cells, 27 (12), 2992-3000.

(80) Zhu, S., Li, W., Zhou, H. et al. (2010) Reprogramming of human primary somatic cells by OCT4 and chemical compounds. Cell Stem Cell, 7 (6), 651-655.

(81) Friedenstein, A.J., Chailakhyan, R.K., Latsinik, N.V. et al. (1974) Stromal cells responsible for transferring the microenvironment of the hemopoietic tissues. Cloning in vitro and retransplantation in vivo. Transplantation, 17 (4), 331-340.

(82) Boquest, A.C., Shahdadfar, A., Fronsdal, K. et al. (2005) Isolation and transcription profiling of purified uncultured human stromal stem cells: alteration of gene expression after in vitro cell culture. Molecular Biology of the Cell, 16 (3), 1131-1141.

(83) Zvaifler, N.J., Marinova-Mutafchieva, L., Adams, G. et al. (2000) Mesenchymal precursor cells in the blood of normal individuals. Arthritis Research, 2 (6), 477-488.

(84) Young, H.E., Steele, T.A., Bray, R.A. et al. (2001) Human reserve pluripotent mesenchymal stem cells are present in the connective tissues of skeletal muscle and dermis derived from fetal, adult, and geriatric donors. Anatomical Record, 264 (1), 51-62.

(85) Bosch, P., Musgrave, D.S., Lee, J.Y. et al. (2000) Osteoprogenitor cells within skeletal muscle. Journal of Orthopaedic Research, 18 (6), 933-944.

(86) Caplan, A.I. and Correa, D. (2011) The MSC: an injury drugstore. Cell Stem Cell, 9(1), 11-15.

(87) Kang, S.K., Shin, I.S., Ko, M.S. et al. (2012) Journey of mesenchymal stem cells for homing: strategies to enhance efficacy and safety of stem cell therapy. Stem Cells International, 2012, 342968.

(88) Xu, Y., Shi, Y., and Ding, S. (2008) A chemical approach to stem-cell biology and regenerative medicine. Nature, 453 (7193), 338-344.

(89) Pittenger, M.F., Mackay, A.M., Beck, S.C. et al. (1999) Multilineage potential of adult human mesenchymal stem cells. Science, 284 (5411), 143-147.

(90) Wu, X., Walker, J., Zhang, J. et al. (2004) Purmorphamine induces osteogenesis by activation of the hedgehog signaling pathway. Chemistry \& Biology, 11 (9), 1229-1238.

(91) Wu, X., Ding, S., Ding, Q. et al. (2002) A small molecule with osteogenesis-inducing activity in multipotent mesenchymal progenitor cells. Journal of the American Chemical Society, 124 (49), 14520-14521.

(92) Xiong, C., Xie, C.Q., Zhang, L. et al. (2005) Derivation of adipocytes from human embryonic stem cells. Stem Cells and Development, 14 (6), 671-675.

(93) Centers for Disease Control and Prevention (2010) CDC and the Arthritis Foundation Launch the 1st National Agenda for Osteoarthritis to Help Millions. National Center for Chronic 
Disease Prevention and Health Promotion, Division of Population Health. Available from: http://www.cdc.gov/arthritis/osteoarthritis.htm (last accessed March 10, 2014).

(94) Grogan, S.P., Miyaki, S., Asahara, H. et al. (2009) Mesenchymal progenitor cell markers in human articular cartilage: normal distribution and changes in osteoarthritis. Arthritis Research \& Therapy, 11 (3), R85.

(95) Lairson, L.L., Lyssiotis, C.A., Zhu, S., and Schultz, P.G. (2013) Small molecule-based approaches to adult stem cell therapies. Annual Review of Pharmacology and Toxicology, 53, 107-125.

(96) Brey, D.M., Motlekar, N.A., Diamond, S.L. et al. (2011) High-throughput screening of a small molecule library for promoters and inhibitors of mesenchymal stem cell osteogenic differentiation. Biotechnology and Bioengineering, 108 (1), 163-174.

(97) Alves, H., Dechering, K., Van Blitterswijk, C., and DeBoer, J. (2011) High-throughput assay for the identification of compounds regulating osteogenic differentiation of human mesenchymal stromal cells. PLoS One, 6 (10), e26678.

(98) Park, K.W., Waki, H., Kim, W.K. et al. (2009) The small molecule phenamil induces osteoblast differentiation and mineralization. Molecular and Cellular Biology, 29 (14), 3905-3914.

(99) Wu, W., Ye, Z., Zhou, Y., and Tan, W.S. (2011) AICAR, a small chemical molecule, primes osteogenic differentiation of adult mesenchymal stem cells. International Journal of Artificial Organs, 34 (12), 1128-1136.

(100) Chen, D.F., Zhang, H.L., Du, S.H. et al. (2010) Cholesterol myristate suppresses the apoptosis of mesenchymal stem cells via upregulation of inhibitor of differentiation. Steroids, 75 (13-14), $1119-1126$.

(101) Saraswati, S., Deskins, D.L., Holt, G.E., and Young, P.P. (2012) Pyrvinium, a potent small molecule Wnt inhibitor, increases engraftment and inhibits lineage commitment of mesenchymal stem cells (MSCs). Wound Repair and Regeneration, 20 (2), 185-193.

(102) Radice, M., Brun, P., Cortivo, R. et al. (2000) Hyaluronan-based biopolymers as delivery vehicles for bone-marrow-derived mesenchymal progenitors. Journal of Biomedical Materials Research, $\mathbf{5 0}$ (2), 101-109.

(103) Benoit, D.S., Schwartz, M.P., Durney, A.R., and Anseth, K.S. (2008) Small functional groups for controlled differentiation of hydrogel-encapsulated human mesenchymal stem cells. Nature Materials, 7 (10), 816-823.

(104) Dahlberg, A., Delaney, C., and Bernstein, I.D. (2011) Ex vivo expansion of human hematopoietic stem and progenitor cells. Blood, 117 (23), 6083-6090.

(105) Peled, T., Landau, E., Mandel, J. et al. (2004) Linear polyamine copper chelator tetraethylenepentamine augments long-term ex vivo expansion of cord blood-derived CD34 + cells and increases their engraftment potential in NOD/SCID mice. Experimental Hematology, 32 (6), 547-555.

(106) de Lima, M., McMannis, J., Gee, A. et al. (2008) Transplantation of ex vivo expanded cord blood cells using the copper chelator tetraethylenepentamine: a phase I/II clinical trial. Bone Marrow Transplantation, 41 (9), 771-778.

(107) Boitano, A.E., Wang, J., Romeo, R. et al. (2010) Aryl hydrocarbon receptor antagonists promote the expansion of human hematopoietic stem cells. Science, 329 (5997), 1345-1348.

(108) Varnum-Finney, B., Brashem-Stein, C., and Bernstein, I.D. (2003) Combined effects of Notch signaling and cytokines induce a multiple log increase in precursors with lymphoid and myeloid reconstituting ability. Blood, 101 (5), 1784-1789.

(109) Delaney, C., Heimfeld, S., Brashem-Stein, C. et al. (2010) Notch-mediated expansion of human cord blood progenitor cells capable of rapid myeloid reconstitution. Nature Medicine, 16 (2), 232-236.

(110) Dallas, M.H., Varnum-Finney, B., Martin, P.J., and Bernstein, I.D. (2007) Enhanced T-cell reconstitution by hematopoietic progenitors expanded ex vivo using the Notch ligand Delta1. Blood, 109 (8), 3579-3587.

(111) North, T.E., Goessling, W., Walkley, C.R. et al. (2007) Prostaglandin E2 regulates vertebrate haematopoietic stem cell homeostasis. Nature, 447 (7147), 1007-1011. 


\title{
7
}

\section{Chemically Induced Pluripotent Stem Cells (CiPSCs): A Potential Chemical Biological Breakthrough in Reprogramming?}

\author{
Calvin C. Sheng ${ }^{1}$, Jijun $\mathrm{Hao}^{2}$, and Charles C. Hong ${ }^{3}$ \\ ${ }^{1}$ Department of Cardiovascular Medicine, Vanderbilt University, USA \\ ${ }^{2}$ College of Veterinary Medicine, Western University of Health Sciences, USA \\ ${ }^{3}$ Department of Medicine, Vanderbilt University, USA
}

\subsection{Searching for the "Perfect" Platform}

The concept of "stem cells" first emerged in the 1960s when transplanted mouse marrowderived cell colonies were shown to have the ability to differentiate into three hematologic lineages: erythrocytes, granulocytes, and megakaryocytes [1]. After this, researchers began searching for the progenitor cells responsible for generating all other cell types during embryonic development. It was not until the early 1980s that mouse embryonic stem cells (mESCs) were first successfully isolated and established as in vitro cultures [2,3]. In the past 3 decades, newer platforms, including human embryonic stem cells (hESCs) [4], human induced pluripotent stem cells (hiPSCs) [5], and, most recently, chemically induced pluripotent stem cells (CiPSCs) [6], have been developed as tools for disease modeling, drug discovery, and regenerative medicine.

Each platform provided a promising glimpse of what the revolutionizing field of stemcell research had to offer in terms of therapeutics and translational medicine. However,

Chemical Biology in Regenerative Medicine: Bridging Stem Cells and Future Therapies, First Edition. Edited by Charles C. Hong, Ada S. Ao, and Jijun Hao.

(C) 2014 John Wiley \& Sons, Ltd. Published 2014 by John Wiley \& Sons, Ltd. 
they all share a common set of technical challenges: how to develop a renewable and efficient source of pluripotent cells, maintain a stable population of pluripotent cells in the undifferentiated state without accumulating genetic abnormalities, and guide differentiation to yield a homogenous somatic cell population. In this chapter, we will focus on a discussion of CiPSCs, the most recent technology (reported in July 2013), and their advantages, disadvantages, and application in regenerative medicine [6].

\subsection{Defining the Advantages of Small Molecules in Reprogramming}

Pluripotent stem cells can be derived through either direct harvesting of embryonic stem cells (ESCs) or, more recently, reprogramming of somatic cells. The latter was first achieved by Yamanaka's group using a cocktail of four transcription factors (Oct3/4, Sox2, Klf4, and c-Myc) [5, 7], circumventing the ethical dilemma of destroying the fertilized embryos associated with ESCs and providing an autologous source of stem cells less prone to immune rejection after transplant. However, hiPSCs must still overcome several limitations, such as low cellular reprogramming efficiency, incomplete reprogramming resulting in heterogeneous population of cells, and risk of tumorigenesis [8]. In particular, c-Myc and Klf4 are known tumorigenic proteins, whose interactions with p53 lead to genomic instability. Within the last year, one study has reported successful reprogramming of murine somatic cells into CiPSCs using only small molecules [6].

This replacement of transcription factors with small molecules offers several distinct advantages during traditional reprogramming and differentiation methods (Table 7.1). First, small molecules are much more cost-effective than biological reagents, and exhibit consistent potency and stability [9]. Second, small molecules can act as reversible modulators, allowing for temporal regulation with high precision [10]. Genetic approaches, by contrast, involve permanent genome modifications with attendant problems of tumorigenicity and other irreversible, unintended consequences. Temporal regulation is also an issue with biological reagents in regards to the duration of effects in in vivo models. Even newer reagents, such as small interfering RNAs (siRNAs), typically function as genetic switches in an allor-none manner, leaving little room for procedural fine-tuning. The final and most crucial point, which is especially germane to the reprogramming efforts, is that cell-permeable small molecules have the potential to target every class of macromolecule in the cell. By contrast, biological reagents are best suited to targeting extracellular components. While

Table 7.1 Comparison of available stem-cell technologies

\begin{tabular}{lccccccc}
\hline & $\begin{array}{c}\text { Established } \\
\text { mouse line }\end{array}$ & $\begin{array}{c}\text { Established } \\
\text { human line }\end{array}$ & $\begin{array}{c}\text { Autologous } \\
\text { cells }\end{array}$ & $\begin{array}{c}\text { Easy to } \\
\text { obtain }\end{array}$ & $\begin{array}{c}\text { Safe, } \\
\text { robust, and } \\
\text { cost-effective }\end{array}$ & $\begin{array}{c}\text { Disease } \\
\text { modeling }\end{array}$ & $\begin{array}{c}\text { Regenerative } \\
\text { medicine }\end{array}$ \\
\hline CiPSCs & $\checkmark$ & $\times$ & $\checkmark$ & $\checkmark$ & $?$ & $?$ & $?$ \\
iPSCs & $\checkmark$ & $\checkmark$ & $\checkmark$ & $\checkmark$ & $?$ & $\checkmark$ & $?$ \\
ESCs & $\checkmark$ & $\checkmark$ & $\times$ & $\times$ & $\times$ & $\checkmark$ & $?$ \\
\hline
\end{tabular}


extracellular signals invariably influence pluripotency, modulation of extracellular signals alone is insufficient to regulate reprogramming events in the nucleus.

\subsection{Understanding the Disadvantages of Using Small Molecules}

One of the main drawbacks to using small molecules is that our current database of bioactive compounds and their cellular effects is far from comprehensive. We do not have a complete database of validated compounds that specifically target every cellular component and pathway. Furthermore, because many small molecules are not truly specific and may have multiple targets, it is more difficult to define a precise mechanism of action. Another concern is that some of the chemicals used in the reprogramming process directly impact the cells at the epigenetic level, leading to potential chemically induced mutations [11]. For instance, BayK (a specific L-type calcium channel agonist) in the presence of BIX (a G9a methyltransferase inhibitor) successfully replaced Sox 2 and c-Myc during reprogramming of mouse embryonic fibroblasts, but BIX as a key regulator of DNA methylation and transcriptional silencing may concomitantly reactivate undesirable oncogenic genes (Table 7.2) [12].

\subsection{Breakthrough: The CiPSC Paradigm}

Yamanaka's retroviral method remains the most efficient and the most widely used, with a transduction reprogramming efficiency of $0.01 \%$ [7]. The use of small molecules has had a tremendous impact in the stem-cell field for two particular reasons: (i) its mimicry of biological reagents in reprogramming, maintaining pluripotency, and guiding differentiation of cells; and (ii) its ability to significantly increase the efficiency of these processes without compromising genomic integrity or intrinsic cellular properties. Over the past several years there has been a progressive trend toward replacing Yamanaka's transcription factors with a defined small-molecule cocktail, alone or with biological reagents, to enhance the efficiency and kinetics of reprogramming [6, 8, 12-18].

A combination of three small molecules - SB431542 (an ALK5 inhibitor), PD0325901 (a mitogen-activated protein kinase kinase (MEK) inhibitor), and thiazovivin (a Rhoassociated kinase inhibitor) - was reported to improve hiPSC reprogramming efficiency by an astounding 200-fold while retaining hESC characteristics (Table 7.2) [8]. In another study, valproic acid (VPA (a histone deacetylase (HDAC) inhibitor) was shown to improve reprogramming efficiency by more than 100-fold (Table 7.2) [13]. Another study that used PS48 ( 3 '-phosphoinositide-dependent kinase-1 activator) alone enhanced reprogramming efficiency by about 15 -fold, and by over 25 -fold when it was combined with sodium butyrate (NaB, an HDAC inhibitor) (Table 7.2) [19]. It can be inferred from these results that certain small molecules not only aid in the reprogramming process but may also be essential to it. Identifying the optimal combination will be the ultimate goal.

In a recent, potentially revolutionary study, Hou et al. demonstrated that pluripotent stem cells could be generated from several mouse somatic cells with a core combination of four small molecules (FSK, CHIR99021, 616452, and DZNep) (Figure 7.1). The same group had demonstrated previously that pluripotent stem-cell induction could be achieved 
Table 7.2 Small molecules shown to have enhanced reprogramming efficiency

Small-molecule compound Chemical structure
BIX01294 [24]

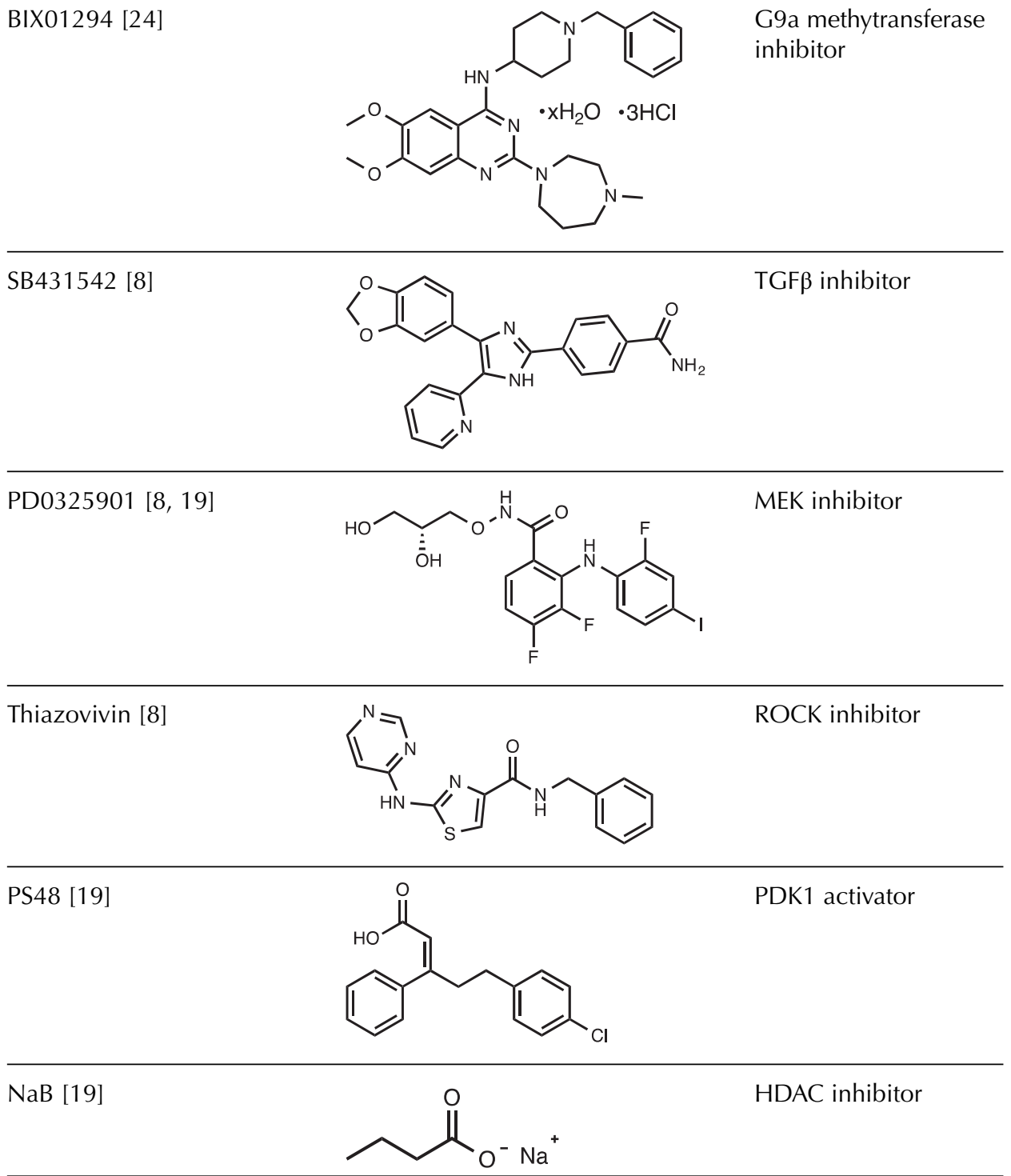

G9a methytransferase inhibitor 


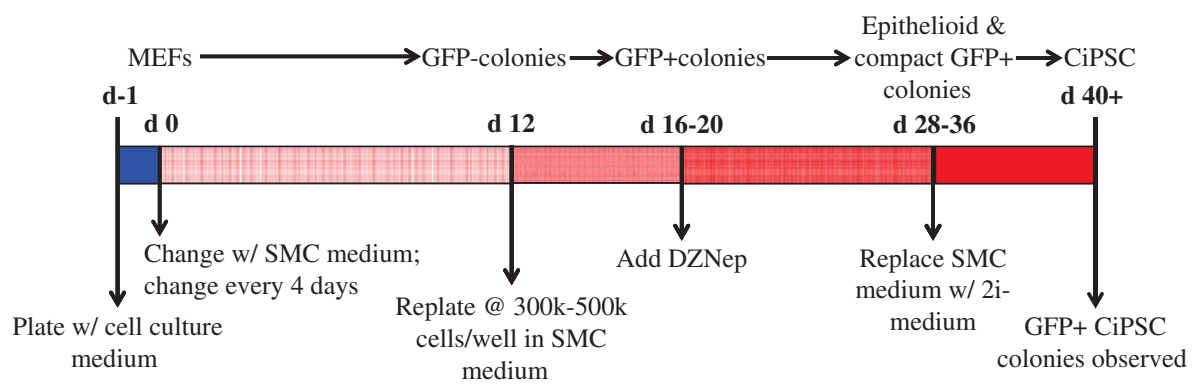

Figure 7.1 Schematic timeline of the CiPSC reprogramming protocol [6]

with a combination of VPA, CHIR99021, 616452, and Tranylcypromine, in addition to an exogenous Oct4 [20]. After screening 10 000 small molecules from multiple libraries, the reprogramming efficiency of mouse embryonic fibroblasts (MEFs) to CiPSCs was increased to $0.2 \%$, comparable to transcription factor-induced reprogramming, using a combination of seven small molecules (VPA, CHIR99021, 616452, Tranylcypromine, FSK, TTNPB, and DZNep) (Table 7.3) [6]. Using this combination, they were able to successfully generate CiPSC lines from mouse neonatal fibroblasts, mouse adult fibroblasts, adipose-derived stem cells, and MEFs.

When characterized, these CiPSCs shared many similarities with ESCs in terms of their gene-expression profile (pluripotency markers detected by immunofluorescence and RTPCR), epigenetic status, and capacity to differentiate into tissues associated with all three germ layers (endoderm, mesoderm, and ectoderm). CiPSC colonies were first isolated 44 days after induction. They maintained normal karyotype and genetic integrity for up to 13 passages [6]. Chimeric immune-deficient (severe combined immunodeficiency, SCID) mice injected with CiPSCs were 100\% viable and healthy for up to 6 months, with no signs of teratogenicity.

A key finding of Hou's study was the elucidation of the role of DZNep, which is a known S-adenosyl-L-homocysteine (SAH) hydrolase inhibitor that functions to modulate the expression of Oct4, a master switch governing pluripotency, at the epigenetic level [6]. It is notable that small molecules proved to be crucial for tight temporal control of the epigenetic modifications necessary for cellular reprogramming, an effort analogous to reverse engineering the epigenetic modifications associated with cell differentiation during embryogenesis. For instance, VPA and Tranylcypromine are two small molecules used to modify the epigenome during the initial stage of reprogramming, but by different mechanism (Figure 7.1). VPA, an HDAC inhibitor, effectively replaces the transcription factor c-Myc, whereas tranylcypromine, a H3K4 demethylation inhibitor, replaces the transcription factor Sox2 in conjunction with CHIR99021, a glycogen synthase kinase 3 (GSK3) inhibitor $[13,17]$. Then, after 16 days, DZNep, another histone methylation inhibitor, is administered to decrease chromatin methylation (Figure 7.1) [21]. DZNep specifically reduces histone $\mathrm{H} 3$ lysine 9 (H3K9) methylation in the promoter region of the Oct4 gene, which activates it [22]. By inducing endogenous Oct4 expression, DZNep effectively overcomes the final epigenetic obstacle to cellular reprogramming. Thus, a timely addition of selective small-molecule epigenetic modifiers is central to establishing pluripotency without the need for exogenous transcription factors. 
Table 7.3 Small molecules used by Hou et al. [6] to replace all four of Yamanaka's transcription factors

\begin{tabular}{|c|c|c|}
\hline Small-molecule compound & Chemical structure & Mechanism of action \\
\hline VPA [13] & & HDAC inhibitor \\
\hline CHIR99021 $[17,25,26]$ & & GSK3 inhibitor \\
\hline $616452[6]$ & & TGF $\beta$ inhibitor \\
\hline Tranylcypromine [6] & & $\begin{array}{l}\text { LSD1 inhibitor/H3K4 } \\
\text { demethylation } \\
\text { inhibitor }\end{array}$ \\
\hline FSK [6] & & cAMP agonist \\
\hline DZNep [6] & & $\begin{array}{l}\text { SAH hydrolase } \\
\text { inhibitor }\end{array}$ \\
\hline TTNPB [6] & & RAR agonist \\
\hline
\end{tabular}


A crucial issue is whether the chemicals alone can be used to generate human CiPSCs. To date, no such publication has been reported, and replicating the success observed in the mouse model may not be straightforward. For instance, an important point of divergence is the difference in pluripotency and epigenetic states between mouse and human stem cells $[4,9]$. A chemical approach incorporating the latest concepts in the difference between "naïve" pluripotent stem cells, presumably representing mESCs, and "primed" pluripotent stem cells could lead to the highly anticipated breakthrough of being able to make human CiPSCs efficiently and inexpensively. While both murine and human models can be utilized for the study of the molecular pathogenesis of diseases, establishing the latter will be imperative to advancing in the expanding field of "human biology", including the development of iPSC models of human diseases. Of course, an efficient and cost-effective approach to deriving human CiPSCs will be invaluable to advancing the regenerative medicine field, providing a renewable source of autologous stem cells.

\subsection{Conclusion}

While "regenerative medicine" is now a topic of constant media exposure and common clinical jargon, the term did not exist until 1992, when it was introduced as follows: "a new branch of medicine will develop that attempts to change the course of chronic disease and in many instances will regenerate tired and failing organ systems" [23]. The costeffectiveness and scalability of small molecules are appealing attributes in regenerative medicine, because they may solve the practical financial burden involved in generating adequate quantities of tissue for autologous transplantation. Small molecules also pave the way for in situ reprogramming and differentiation, since they are much less immunogenic than biological reagents and can be quickly discontinued after therapeutic application. Nonetheless, the use of small molecules to generate mouse CiPSCs is only the first step in decreasing the reliance on biological reagents, despite being hailed as a potential "holy grail of regenerative medicine" by some stem-cell experts and enthusiasts. Future studies are required to determine whether this approach is feasible in human cells and, if so, to define the optimal combinations of small molecules for efficient reprogramming of human cells and for directed differentiation of human CiPSCs into desired tissue types.

\section{References}

(1) Becker, A.J., McCulloch, E.A., and Till, J.E. (1963) Cytological demonstration of the clonal nature of spleen colonies derived from transplanted mouse marrow cells. Nature, 197, 452-454.

(2) Martin, G.R. (1981) Isolation of a pluripotent cell line from early mouse embryos cultured in medium conditioned by teratocarcinoma stem cells. Proceedings of the National Academy of Sciences, 78 (12), 7634-7638.

(3) Evans, M.J. and Kaufman, M.H. (1981) Establishment in culture of pluripotential cells from mouse embryos. Nature, 292 (5819), 154-156.

(4) Thomson, J.A., Itskovitz-Eldor, J., Shapiro, S.S. et al. (1998) Embryonic stem cell lines derived from human blastocysts. Science, 282 (5391), 1145-1147.

(5) Takahashi, K., Tanabe, K., Ohnuki, M. et al. (2007) Induction of pluripotent stem cells from adult human fibroblasts by defined factors. Cell, $\mathbf{1 3 1}$ (5), 861-872. 
(6) Hou, P., Li, Y., Zhang, X. et al. (2013) Pluripotent stem cells induced from mouse somatic cells by small-molecule compounds. Science, 341 (6146), 651-654.

(7) Takahashi, K. and Yamanaka, S. (2006) Induction of pluripotent stem cells from mouse embryonic and adult fibroblast cultures by defined factors. Cell, 126 (4), 663-676.

(8) Lin, T., Ambasudhan, R., Yuan, X. et al. (2009) A chemical platform for improved induction of human iPSCs. Nature Methods, 6 (11), 805-808.

(9) Ao, A., Hao, J., and Hong, C.C. (2011) Regenerative chemical biology: current challenges and future potential. Chemistry \& Biology, 18 (4), 413-424.

(10) Hao, J., Sawyer, D.B., Hatzopoulos, A.K. et al. (2011) Recent progress on chemical biology of pluripotent stem cell self-renewal, reprogramming and cardiomyogenesis. Recent Patents on Regenerative Medicine, 1 (3), 263.

(11) Solanki, A. and Lee, K.B. (2010) A step closer to complete chemical reprogramming for generating iPS cells. ChemBioChem, 11 (6), 755-757.

(12) Shi, Y., Desponts, C., Do, J.T. et al. (2008) Induction of pluripotent stem cells from mouse embryonic fibroblasts by Oct4 and Klf4 with small-molecule compounds. Cell Stem Cell, 3 (5), $568-574$.

(13) Huangfu, D., Osafune, K., Maehr, R. et al. (2008) Induction of pluripotent stem cells from primary human fibroblasts with only Oct4 and Sox2. Nature Biotechnology, 26 (11), 12691275.

(14) Lyssiotis, C.A., Foreman, R.K., Staerk, J. et al. (2009) Reprogramming of murine fibroblasts to induced pluripotent stem cells with chemical complementation of Klf4. Proceedings of the National Academy of Sciences, 106 (22), 8912-8917.

(15) Ichida, J.K., Blanchard, J., Lam, K. et al. (2009) A Small-Molecule Inhibitor of Tgf-beta Signaling Replaces Sox2 in Reprogramming by Inducing Nanog. Cell Stem Cell, 5 (5), 491503.

(16) Maherali, N. and Hochedlinger, K. (2009) Tgf-beta signal inhibition cooperates in the induction of iPSCs and replaces Sox2 and cMyc. Current Biology, 19 (20), 1718-1723.

(17) Li, W., Zhou, H., Abujarour, R. et al. (2009) Generation of human-induced pluripotent stem cells in the absence of exogenous Sox2. Stem Cells, 27 (12), 2992-3000.

(18) Esteban, M.A., Wang, T., Qin, B. et al. (2010) Vitamin C enhances the generation of mouse and human induced pluripotent stem cells. Cell Stem Cell, 6 (1), 71-79.

(19) Zhu, S., Li, W., Zhou, H. et al. (2010) Reprogramming of human primary somatic cells by OCT4 and chemical compounds. Cell Stem Cell, 7 (6), 651-655.

(20) Li, Y., Zhang, Q., Yin, X. et al. (2010) Generation of iPSCs from mouse fibroblasts with a single gene, Oct4, and small molecules. Cell Research, 21 (1), 196-204.

(21) Miranda, T.B., Cortez, C.C., Yoo, C.B. et al. (2009) DZNep is a global histone methylation inhibitor that reactivates developmental genes not silenced by DNA methylation. Molecular Cancer Therapeutics, 8 (6), 1579-1588.

(22) Chen, J., Liu, H., Liu, J. et al. (2012) H3K9 methylation is a barrier during somatic cell reprogramming into iPSCs. Nature Genetics, 45 (1), 34-42.

(23) Kaiser, L. (1992) The future of multihospital systems. Topics in Health Care Financing, 18 (4), 32.

(24) Shi, Y., Do, J.T., Desponts, C. et al. (2008) A combined chemical and genetic approach for the generation of induced pluripotent stem cells. Cell Stem Cell, 2 (6), 525-528.

(25) Ying, Q.-L., Wray, J., Nichols, J. et al. (2008) The ground state of embryonic stem cell selfrenewal. Nature, 453 (7194), 519-523.

(26) Li, W., Wei, W., Zhu, S. et al. (2009) Generation of rat and human induced pluripotent stem cells by combining genetic reprogramming and chemical inhibitors. Cell Stem Cell, 4 (1), 16-19. 


\title{
8 \\ An Introduction to Cellular Reprogramming: The Plasticity of Cell Fates and Identities
}

\author{
Kelly P. Smith ${ }^{1}$, Maria Borowski ${ }^{1}$, and Joseph C. Laning ${ }^{2}$ \\ ${ }^{1}$ Department of Cell and Developmental Biology, University of Massachusetts \\ Medical School, USA \\ ${ }^{2}$ Research and Development, Provia Laboratories, LLC, USA
}

Since the pioneering studies on nuclear transplantation by John Gurdon [1] and the identification of bone-marrow stem cells by James Till and Ernest McCulloch [2], the last 50+ years have seen remarkable progress in stem-cell science (Figure 8.1). Adult stem cells have been isolated and cultured from numerous tissues and pluripotent stem cells from early embryos (embryonic stem cells, ESCs) have been successfully isolated, cultured, and differentiated into many different cell types. These breakthroughs have allowed researchers to delve into the molecular mechanisms and pathways unique to stem cells that control their ability to develop into numerous cell types. Such fundamental studies have resulted in the current wave of technical innovations that has allowed scientists to "reprogram" adult somatic cells to a state of pluripotency similar to ESCs (induced pluripotent stem cells, iPSCs) [3] or to convert cells from one type to another (direct lineage conversion or transdifferentiation) [4]. These reprogramming technologies are transforming biomedical science, from providing tools for the study of development and disease, to enabling the creation of patient-specific cells for the treatment of a broad range of conditions.

Chemical Biology in Regenerative Medicine: Bridging Stem Cells and Future Therapies, First Edition. Edited by Charles C. Hong, Ada S. Ao, and Jijun Hao.

(C) 2014 John Wiley \& Sons, Ltd. Published 2014 by John Wiley \& Sons, Ltd. 


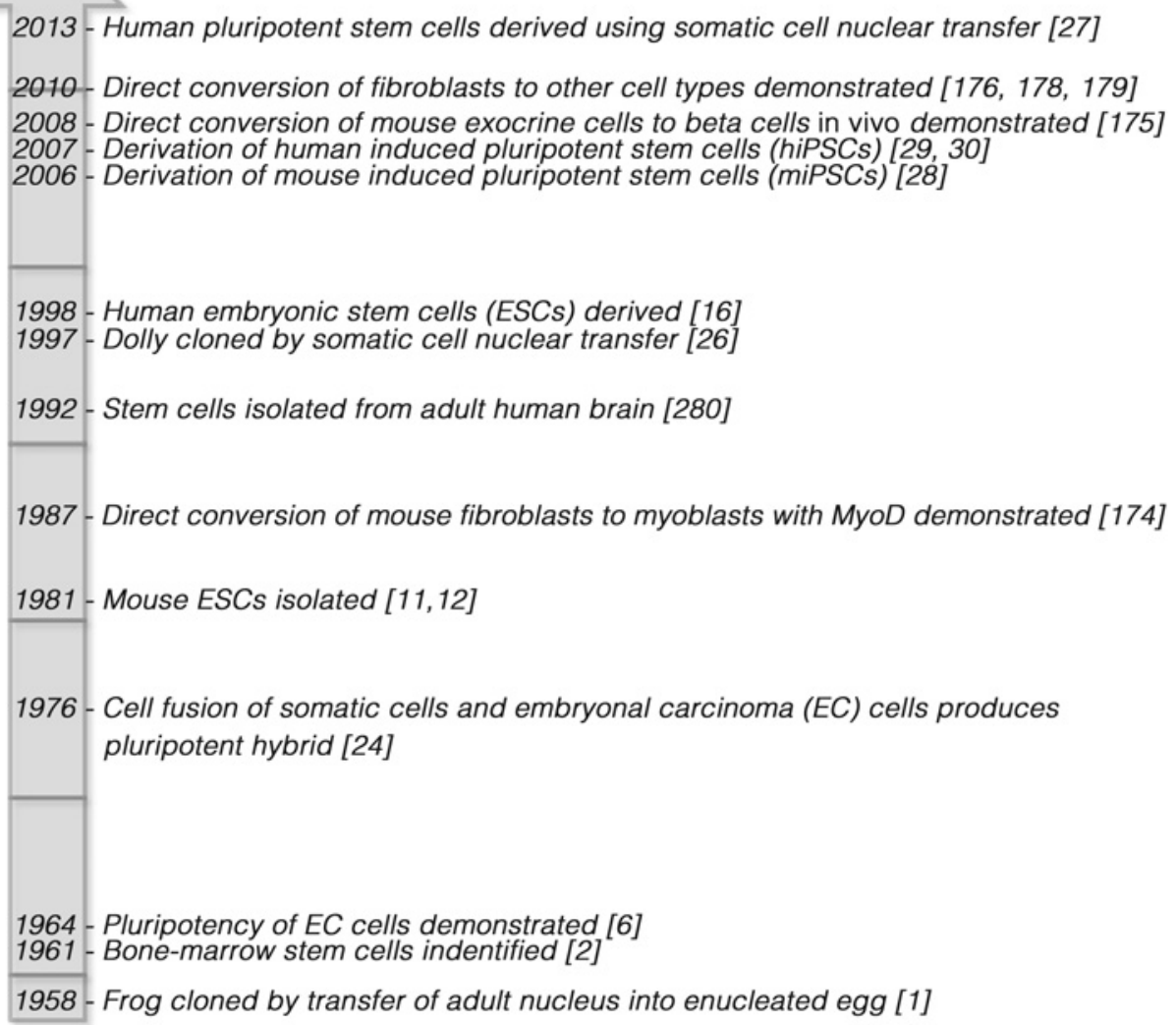

Figure 8.1 Stem cell research timeline

\subsection{Defining Cell Potency}

As an organism develops, stem cells gradually lose their differentiation capacity. The fertilized egg (zygote) and the blastomeres of the four cell-stage morula are totipotent: capable of developing into any embryonic or extraembryonic cell type [5]. Cells making up the blastocyst inner cell mass (ICM) are pluripotent, with the ability to differentiate into any of over 200 cell types found in the mature organism. Beyond this stage, fetal and adult stem cells become more lineage-restricted (multipotent) in their differentiation capacity. The final stem-cell stage is the development of precursor cells, which can change into a very limited set of related terminally differentiated somatic cell types.

In addition to their capacity for differentiation, pluripotent cells are capable of unlimited growth. These characteristics allow pluripotent cells to be expanded indefinitely and differentiated to create large quantities of any cell type. This capability makes pluripotent cells an enormously important tool. Cells can be followed through the differentiation process, allowing researchers to investigate development, in both normal and disease states, in new 
ways. Additionally, the creation of large quantities of specific cell types has applications ranging from drug and toxicity screening to cellular therapy and tissue engineering.

\subsection{Types of Pluripotent Cell}

\subsubsection{Isolated Cell Types}

\subsubsection{Embryonal Carcinoma Cells}

Embryonal carcinoma (EC) cells are derived from teratocarcinomas or malignant germ-cell tumors. These tumors possess a mixture of cell types representing all three germ layers as well as malignant undifferentiated EC cells. EC cells extracted from these tumors have been shown to have the capacity to self-renew and differentiate into multiple cell lineages, establishing the concept of the pluripotent cell [6]. EC cell lines were subsequently derived from mouse [7] and human [8]. Although considered pluripotent, EC cells are malignant in the undifferentiated state, are often karyotypically abnormal [9], and have a limited capacity to differentiate [10]. Nonetheless, study of the characteristics of EC cells, how they are cultured, how mouse and human EC cells differ, and how they differentiate has laid the foundation for subsequent advances in stem-cell and developmental biology.

\subsubsection{Embryonic Stem Cells}

ESCs are derived by the isolation of cells from the ICM of a blastocyst-stage embryo and adapting them to in vitro growth. ESCs from mouse embryos were first successfully isolated and cultured in 1981 [11,12]. These mouse ESCs were shown to have the capacity to differentiate into the three germ lineages in vitro by embryoid body formation and to form teratomas when injected into mice. More importantly, pluripotency of these cells was established by their ability to form germ-line chimeras when reintroduced into a mouse blastocyst embryo [13]. The most definitive demonstration of pluripotency was done by injection of mouse ESCs into tetraploid (4N) blastocysts, which are incapable of normal development. This system allows the generation of mice derived completely from the injected ESCs $[14,15]$.

It was not until 1998 that human embryonic stem cells (hESCs) were successfully derived [16]. Although hESCs are similar to mouse embryonic stem cells (mESCs) in many respects, they differ in colony morphology and growth conditions. hESCs differentiate into three germ layers through embryoid body formation [17] and in teratomas [16], but ethical constraints make chimera and tetraploid complementation studies impossible. As discussed later, the true state of pluripotency in hESCs has been the subject of considerable study.

\subsubsection{Embryonic Germ Cells}

Embryonic germ cells (EGCs) are derived from the primordial germ cells (PGCs) found in fetal gonads. PGCs are unipotent, in that they are progenitors of sperm and egg cells via meiosis. These cells cannot self-renew or survive in culture under standard conditions. However, under specific growth conditions mouse [18, 19] and human [20,21] EGCs have been derived from these primordial cells. Unlike PGCs, EGCs are capable of selfrenewal and of forming cell types representing the three germ layers. However, EGCs are 
difficult to maintain in an undifferentiated state in culture and spontaneously form embryoid bodies. Unlike other pluripotent cells, they cannot form teratomas in nude mice and have a gene-expression signature that is distinct from other pluripotent cell types [22]. EGCs are morphologically similar to mESCs and express the stem-cell marker SSEA-1, which is expressed in mESCs but not hESCs. Thus, it has been suggested that EGCs may exist in some unique or intermediate state of pluripotency.

\subsubsection{Reprogrammed Cell Types}

\subsubsection{Cell-Fusion Hybrids}

The plasticity of differentiated cells is evident from cell-fusion studies [23]. In 1974, it was shown that fusion of embryonic carcinoma cells and thymocytes resulted in hybrid cells in which the pluripotent phenotype was maintained [24]. Similarly, hESCs have been fused with human fibroblasts to derive stable tetraploid hybrids that retain the pluripotent characteristics of the ESCs [25]. Cell-fusion hybrids are used primarily to investigate the mechanisms of reprogramming and pluripotency.

\subsubsection{Somatic Cell Nuclear Transfer Cells}

Somatic cell nuclear transfer (SCNT) involves the transfer of a somatic cell nucleus into an unfertilized oocyte. First demonstrated as a method for cloning animals [26], this technique also offered the prospect of deriving patient-specific pluripotent cells by isolating them from the embryo developed from the hybrid SCNT egg. Despite this prospect, fears surrounding reproductive cloning and the legal and social issues around egg donation have slowed progress in this area. Nonetheless, the reprogramming of human somatic cells to pluripotency by nuclear transfer into an oocyte was recently reported, demonstrating that this technique can be used to produce human pluripotent cells [27].

\subsubsection{Induced Pluripotent Stem Cells}

Groundbreaking work first done in mice by Takahashi and Yamanaka [28] established that differentiated fibroblast cells taken from an adult mouse could be reverted to a pluripotentlike state by introduction of specific transcription factors. In this remarkable study, by homologous recombination $\beta$-galactosidase and neomycin resistance genes were incorporated into the Fbx16 gene, which is a nonessential gene expressed in pluripotent stem cells. Using retroviruses, the authors introduced and expressed various combinations of a set of 24 candidate genes identified based on their roles in the maintenance of ESC pluripotency. The result of this inquiry showed that the introduction of four specific transgenes, Oct4, Sox2, Klf4, and c-Myc, could induce fibroblasts to convert to a state similar to ESCs. This result was closely followed by the derivation of human induced pluripotent stem cells (hiPSCs) [29,30]. Since this landmark study, the methods used to reprogram cells have expanded greatly, to include different viruses, episomal delivery, plasmids, proteins, mRNA, miRNAs, and small molecules, as described later. 


\subsection{Defining Pluripotency}

Pluripotency is a complex, dynamic state that has proven difficult to precisely define and measure [31]. Pluripotent cells follow a program of self-renewal while primed to begin a differentiation program in response to specific developmental signals. As such, this state is fairly unstable and there is significant variability in differentiation potential between different pluripotent cell lines [32-34]. Furthermore, it has been found that the pluripotent states of individual ESCs within a culture can vary $[35,36]$. It has been suggested that such variation in pluripotent states could be an integral mechanism of ESCs, where selfrenewal is maintained within the colony or embryo while opportunities for differentiation are explored [37].

Recent studies have uncovered that there are at least two distinct states of pluripotency: the naïve and the primed states, which differ in their epigenetic status and differentiation capacity [38]. Although hESC and mESC are both derived from pre-implantation blastocysts, differences between the two cells in culture are evident. mESCs grow in tightly packed, three-dimensional colonies and have a relatively short doubling time (16 hours); hESCs grow in flatter colonies and their doubling time is more than twice as long (36 hours), and they are maintained with different growth-factor signaling conditions. Furthermore, while mESCs can be passaged as single cells, hESCs must be passaged as clumps, making procedures that require clonal selection, such as genetic modification, much more difficult. Finally, while female hESCs exhibit inactivation of one X chromosome, in mESCs both $\mathrm{X}$ chromosomes are active. This indicates that mESCs may be at a different epigenetic stage than hESCs.

Other studies have isolated mouse epiblast stem cells (epiSCs) from post-implantation embryos that have characteristics much more similar to hESCs than to mESCs $[39,40]$. The gold-standard in vivo functional assay for pluripotency is the development of chimeras by reintroduction of ESCs into an embryo where the ESCs contribute to all cell types in the resulting organism. Interestingly, while mESCs pass this functional test, epiSCs and nonhuman primate ESCs [41] have limited capacity for chimera formation. Thus, although all of these cell types exhibit pluripotency in vitro, it has been suggested that mESCs exist in a more potent "naïve" state, while epiSCs and hESCs are in a more "primed" pluripotent state [38, 42]. More recent studies suggest that pluripotency is a metastable state which can be determined by culture and derivation conditions $[43,44]$ and that hESCs can be derived in conditions [45] or exposed to culture conditions post-derivation [46] that allow them to exhibit functional and molecular characteristics similar to those of mESCs. These differences in pluripotent state are important in that they may influence the differentiation capacity and susceptibility to genetic modification of an ESC or iPSC line.

Demonstration of the pluripotency of ESC lines when they are derived and during continued expansion of the cultures is necessary to ensure that they retain the capacity for differentiation while spontaneous differentiation is inhibited. Determination of pluripotency in iPSCs is especially important as variability in methodologies, reagents, starting materials, and environmental conditions can lead to the development of lines that are not fully reprogrammed $[28,47]$.

There are a number of methods that are used to determine pluripotency in a cell line (Table 8.1). Molecular methods can be used to detect expression of genes known to be expressed in pluripotent cells or to define methylation patterns specific to pluripotent cells. 
Table 8.1 Methods of assessing pluripotency

\begin{tabular}{|c|c|c|}
\hline $\begin{array}{l}\text { Analysis of } \\
\text { pluripotency }\end{array}$ & Tests & Methods \\
\hline \multirow[t]{3}{*}{ Molecular } & $\begin{array}{l}\text { Expression of } \\
\text { pluripotency-specific } \\
\text { genes }\end{array}$ & $\begin{array}{l}\text { Immunocytochemical staining } \\
\text { (marker protein detection) } \\
\text { and/or quantitative RTPCR } \\
\text { (mRNA detection) }\end{array}$ \\
\hline & Whole-genome expression & Microarray hybridization \\
\hline & Epigenetic analysis & $\begin{array}{l}\text { Determination of the degree of } \\
\text { methylation of ESC-specific } \\
\text { gene promoters }\end{array}$ \\
\hline \multirow[t]{2}{*}{ In vitro } & Embryoid body formation & $\begin{array}{l}\text { Spontaneous differentiation of } \\
\text { cell clumps followed by } \\
\text { detection of lineage-specific } \\
\text { markers by ICC or qRTPCR. }\end{array}$ \\
\hline & Directed differentiation & $\begin{array}{l}\text { Differentiation of cultures toward } \\
\text { specific cell types }\end{array}$ \\
\hline \multirow[t]{3}{*}{ In vivo } & Teratoma formation & $\begin{array}{l}\text { Spontaneous differentiation of } \\
\text { cells injected into specific sites } \\
\text { in mice }\end{array}$ \\
\hline & Chimera production & $\begin{array}{l}\text { Introduction of cells into a mouse } \\
\text { blastocyst and detection of } \\
\text { differentiated progeny of the } \\
\text { introduced cells in all tissues of } \\
\text { the adult }\end{array}$ \\
\hline & $\begin{array}{l}\text { Tetraploid } \\
\text { complementation }\end{array}$ & $\begin{array}{l}\text { Introduction of cells into a mouse } \\
4 \mathrm{~N} \text { (tetraploid) blastocyst. } \\
\text { Mature mouse will develop } \\
\text { entirely from introduced cells }\end{array}$ \\
\hline
\end{tabular}

Other techniques demonstrate the cells' capacity to differentiate into specific cell types in vitro or in vivo. While the production of viable adult organisms by the introduction of pluripotent cells into an embryo for chimera formation [48] or by tetraploid (4N) complementation $[14,15]$ may be the most definitive test of pluripotency, this method is clearly not ethical for studies of human pluripotent cells.

\subsection{The Molecular Basis of Pluripotency}

The pluripotent state/s is/are reliant upon a number of interconnected processes, including the cellular environment, growth-factor signaling, transcription-factor networks, noncoding RNAs, and epigenetic modifications $[49,50]$. Current models propose that pluripotency is primarily controlled by the core transcription factors Oct4, Sox2, and Nanog [51]. Oct4 is considered the master regulator of the development and maintenance of pluripotent cells, as Oct4-deficient mouse embryos fail to produce an ICM [52] and suppression of Oct4 in ESCs leads to differentiation along a trophectodermal lineage [53]. Oct4 often 


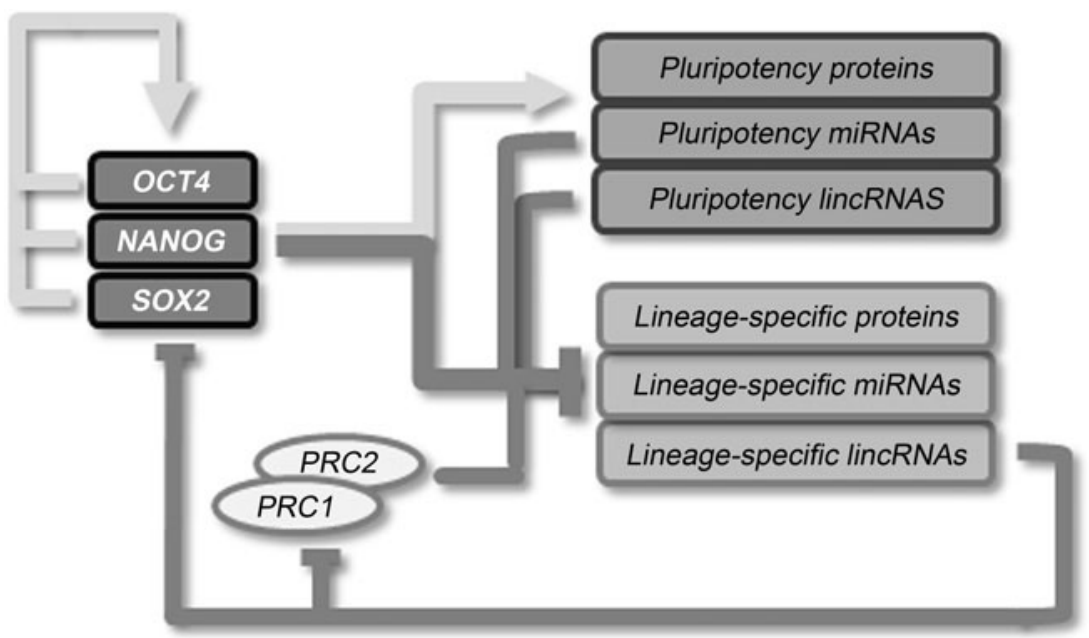

Figure 8.2 The pluripotency network

forms a heterodimer with Sox 2 to bind to the regulatory elements of many different target genes in ESCs [54, 55] and promotes an undifferentiated state in ESCs [56, 57]. Nanog, Oct4, and Sox 2 are reported to co-occupy many target genes in hESCs and function in an interconnected autoregulatory loop to regulate their own expression (Figure 8.2) [51, 53]. These factors also interact with an extended network of other transcription factors to create a regulatory web that promotes pluripotency $[58,59]$.

Interestingly, while these factors bind regulatory sequences to promote transcription, each may also be involved in lineage specification and the repression of lineage-specific genes in ESCs [49, 60]. Overexpression of each of these factors drives differentiation into specific lineages, while knockdown of each factor blocks the same lineage differentiation. While all three factors often co-occupy the regulatory elements of active genes in ESCs, individual factors are frequently bound to repressed loci. Furthermore, active genes are most often bound by multiple factors, while repressed genes are often associated with a single transcription factor $[58,59]$. Core transcription factors have been found to cooccupy repressed loci encoding important developmental regulators with the polycomb repressive complex 2 (PRC2) [61,62]. PRC2 catalyzes the trimethylation of histone H3 at lysine 27 (H3K27me3) to promote gene silencing [63]. Many of these developmental genes exist within "bivalent domains" in the genome, where genes possess histone modifications that promote gene silencing (HK27me3), but reside within larger domains that have histone modifications of active chromatin (H3K4me3) [64]. Such bivalent modifications are thought to allow the important developmental regulators to exist in a "primed" state in ESCs, where RNA polymerase II is loaded on to the promoter but is held in check by the repressive chromatin $[65,66]$. In this state, transcription of lineage-specific regulatory genes can be rapidly activated in response to specific developmental cues.

Another component of the pluripotency control circuitry involves noncoding RNAs. MicroRNAs (miRNAs) are small noncoding RNAs that regulate gene expression by binding to target mRNAs and facilitating their degradation. Numerous miRNAs have been identified 
as being expressed in ESCs, and the expression some of these appears to be regulated by binding of the core transcription factors to their gene promoters [67]. Lineage-specific miRNA genes are also bound by the core factors, but are not expressed due to the presence of polycomb repressor complexes such as PRC2, as observed for lineage-specific, proteincoding genes. In turn, several lineage-specific miRNAs can downregulate expression of the core transcription factors [68], as well as components of polycomb group complexes (Figure 8.2) [62].

The functions of large intergenic noncoding RNAs (lincRNAs) are not well understood, but these evolutionarily conserved RNAs interact with chromatin-modifying complexes and influence gene expression [69]. It has been suggested that the lincRNAs could function as flexible scaffolds, bringing together different protein complexes to regulate gene expression. Numerous ESC-specific lincRNAs have been identified whose expression is regulated by core pluripotency factors [70]. Many of these lincRNAs appear to help maintain the pluripotent state and repress expression of lineage-specific gene expression. LincRNAs may facilitate this repression by binding to chromatin-modifying complexes such as PRC2.

\subsection{Cellular Reprogramming: Altering the Epigenetic State}

The variety of cell types in the adult are created via differentiation. Differentiation is a highly complex and regulated process controlled by transcription-factor networks and epigenetic modification of the genome. Epigenetic modifications to chromatin are carried out by a number of different mechanisms, including DNA methylation, post-translational modification of histones, incorporation of histone variants, noncoding RNAs, RNA interference (RNAi, and ATP-dependent chromatin remodeling [71]. ESCs typically have an open euchromatic chromatin state with little inactive heterochromatin. As these cells undergo differentiation, the epigenetic "landscape" of the chromatin is modified [72]. Such modifications occur in very specific patterns throughout the genome, resulting in regions of DNA that are expressed or repressed, and are unique to a particular cell type. Epigenetic modifications are inherited by successive generations of the cells, ensuring that the cellular specialization is maintained.

Chromatin remodeling, particularly in the bivalent domains corresponding to important transcription-factor genes, is an essential temporal regulatory switch in the reprogramming process [47, 64, 73-77]. The work of Yamanaka's group highlighted an underappreciated aspect of genomics and epigenetic control: the extraordinary plasticity of the mammalian genome to reshape itself as part of the reprogramming process. A vital component in the reprogramming timeline is the resetting of the epigenetic landscape. Stochastic remodeling events occur as a result of transcription-factor overexpression leading to endogenous expression of stem-cell factors required for stable cell-line progression [77, 78]. Immediately upon forced expression of the transfected reprogramming factors, some proportion of cells starts to divide more quickly and continues down a path of directed somatic gene expression downregulation. However, only a subset of cells continue to upregulate the pluripotent expression profile, and this extended expression is essential in the development of a fully reprogrammed cell line $[79,80]$. Finally, continuous endogenous pluripotency 
gene expression establishes a stable ESC-like genomic profile that allows for a sustained functional stem-cell phenotype. This stepwise and multistage process involves so many interconnected pathways and mechanisms that it is not surprising that the path to induced pluripotency is an inefficient one. While several reports have indicated that in most respects human iPSCs and ESCs are globally quite similar, the true depth of the similarity is still being debated at the molecular level. It appears that as analyses become more sophisticated and the detail becomes more refined, the general similarities between ESCs and iPSCs are considerably eroded $[3,76,81-83]$. As with many experimental systems, much of the variability may be generated from differences between investigators relating to cell-culture methods, starting cell populations, reprogramming methods, and screening or characterization techniques. Being different is not necessarily bad, but it does speak to the need for standards and controls by which both research and therapeutic cell lines can be measured before meaningful comparisons can be made.

Stem cells, particularly iPSCs, are widely lauded as powerful tools in the fields of regenerative medicine and the study of developmental biology and disease. However, reprogramming of the epigenome is an inexact process and incomplete reprogramming can result in some of the identity or "epigenetic memory" of the source cell being retained during iPSC derivation [84-86]. This memory effect can influence the differentiation capacity of an iPSC line. However, it has been shown that epigenetic memory can be partially erased via extended culture and that DNA methylation inhibitors can significantly influence the differentiation paths of iPSC lines [82, 85, 87]. Whether epigenetic memory is perceived as a liability or an asset likely depends on the intended use of the reprogrammed cell product. Screening for epigenetic memory through whole-genome sequencing may allow for targeted application of specific cell lines for drug screens or in those cell therapies most suited to the differentiative predisposition of the cell line. On the other hand, one might envision the most completely reprogrammed cell lines being reserved for developmental biology investigations requiring unhindered plasticity. In either case, epigenetic memory might be viewed as an advantageous attribute when developing future tools or therapies.

\subsection{Cellular Reprogramming: Primary Regulatory Pathways}

The advent of cellular reprogramming has demonstrably changed the landscape of stemcell biology, disease modeling, and potentially even cellular therapies and personalized medicine. Simply stated, cellular reprogramming is the forced expression of several pathway-specific transcription factors in an adult somatic cell such that the cellular machinery and epigenetic profile revert to a more primitive stem-cell state. The landmark publication in 2006 of the work of Takahashi and Yamanaka found that the genes they screened (Oct3/4, Sox2, Klf4, and c-Myc) could be combined through retroviral transduction achieving prolonged factor expression in order to produce ESC-like cell lines from mouse somatic cells [28]. Although the delivery of the original four transcription factors through viral transduction remains the most commonly used method to date, work continues to improve the efficiency and ultimate safety of the reprogramming process with an eye toward therapeutics. A portion of this section will be devoted to the creative alternatives being explored to 
achieve this goal. However, our current review will be limited to the four major pathways represented by the original combination of factors.

The four main established transcription factors, Oct4, Sox2, Klf4, and c-Myc (OSKM), represent an interconnected reprogramming cocktail that, when expressed transiently, establishes an embryonic-like environment in the target cell. Once expressed, these factors activate the cell's own endogenous pluripotency pathways, driving the cell toward a stable pluripotent phenotype. Oct4 (Pou5f1), previously mentioned as the "master regulator", is well known as a transcription factor required for early embryonic development and for the maintenance of pluripotency in ESCs. In experiments limiting the number of factors used to generate iPSC lines, Oct4 was found to be essential to the occurrence of complete reprogramming [88-90].

Sox2, a transcription factor in the SRY-related HMG-box family, is directly involved in the determination of defined lineage development and the maintenance of the undifferentiated state for embryonic and neural stem cells (NSCs). Oct4 and Sox 2 can work cooperatively using Sox-Oct motifs that are frequently found within the regulatory sequences of other important pluripotency genes [54]. The expression of many pluripotency-associated genes, including Lefty 1 and Nanog, is regulated by enhancers containing Oct3/4 and Sox 2 binding motifs, which highlights the dominant regulatory role Oct4 and Sox 2 have in maintaining pluripotency [91,92]. Additionally, Sox 2 and Oct4 have been reported to bind to a complex of DNA repair proteins, facilitating the transactivation of Nanog [93]. It has been shown in mouse fibroblasts that Sox 1 and Sox 3 can replace Sox2, although this adversely affects reprogramming efficiency.

Kruppel-like factor 4 (Klf4) is required to establish asymmetrical embryo development, as well as a myriad of roles from gut epithelial polarization to tumorigenesis, immune regulation, and iPSC derivation [94-100]. Interestingly, based on studies by Nakagawa and colleagues in murine cells, Klf2 can substitute for Klf4 in reprogramming-factor combinations. Similarly, Klf1 and Klf5 can also substitute for Klf4, but with significantly lower reprogramming efficiency [88]. DNA-binding studies have revealed several shared targets for Oct4, Sox2, and Klf4 related to the control of pluripotency, including Klf4 and Oct4 co-occupying the Nanog promoter, and in the absence of ecoptopic Klf4 expression, endogenous Klf4 expression is required for the reprogramming of somatic cells [101]. This provides further evidence of the connected functional overlap between Sox 2 and Klf4 in the disruption of cellular homeostasis and activation of regulatory networks that define pluripotency.

The oncogene c-Myc, originally recognized for its role in Burkitt's lymphoma through chromosomal translocation, was also identified as a key regulator in the reprogramming platform enabling iPSC derivations. c-Myc is a key regulator of both cell growth and metabolism, playing a role in both transformation and cell-cycle entry [102,103]. Additionally, c-Myc participates in the maintenance of ESC pluripotency via the Lif-Stat3 pathway and can induce global histone acetylation, allowing Oct4 and Sox 2 to bind to their specific target loci $[104,105]$. Alternatively, it has been suggested that c-Myc amplification of existing gene profiles due to increased c-Myc abundance and favorable epigenetic access allows for the vast array of oncogenic effects seen when c-Myc is overexpressed, as is the case under many reprogramming methods [106]. Also, the specific substitution of c-Myc has been used in the successful reprogramming of human cells by replacement of c-Myc with L-Myc or N-Myc [88]. While it is widely considered that Myc, in any form, is 
important for efficient reprogramming, it has been shown that it is not required for reprogramming of mouse and human fibroblasts [30, 88, 107]. However, subsequent studies have shown that adding back c-Myc significantly augments reprogramming efficiency [108].

Numerous studies continue to explore new twists to the original four-factor combination. For instance, reprogramming has been demonstrated using only Oct4 and Sox2 [109]. Furthermore, Scholer and his colleagues [110] have demonstrated that Oct4 alone was able to convert human NSCs into iPSCs. This strongly suggests a role for the differentiative state of the reprogramming target cell type in the overall ease and efficiency with which a somatic cell converts to an iPSC. Another recent discovery has shown that although Oct4 is essential, it can be replaced with a nuclear receptor gene, Nr5a2, in the derivation of iPSCs from mouse somatic cells [111]. In addition, including the retinoic acid receptor (RAR $\gamma$ ) and $\mathrm{Nr} 5 \mathrm{a} 2$ with the four factors has been shown to greatly increase reprogramming efficiency in both mouse and human cells [112]. While these and other studies show several compelling factor combinations or reductions can be employed in the reprogramming, the primary set of factors still remains the minimally sufficient combination for efficient complete reprogramming, whether that occurs as a result of the expression of the transcription factors alone or in combination with compounds that substitute for one or more of them. Of course, the process remains influenced by the endogenous expression of the core factors or related pathways, as well as the characteristics of the target cell, such as proliferation rate [113].

\subsubsection{Temporal and Stoichiometric Considerations}

The stoichiometry and timing of transcription-factor expression significantly influence the properties of the reprogrammed cells [114] and numerous studies have investigated the temporal and stoichiometric parameters related to one or more of the original reprogramming factors, as well as the potential replacement of certain factors with proteins or small molecules [100]. More recently, studies employing a tagging system using monoclonal antibodies that could be used to analyze the expression levels of the four reprogramming factors in sorted iPSCs found that the most effective ratio (Oct3/4- $\uparrow$, Sox2- $\downarrow$, Klf4- $\uparrow$, c-Myc- $\uparrow$ ) was almost $100 \times$ more efficient at producing iPSCs than the least optimal ratio (Oct3/4- $\downarrow$, Sox2- $\uparrow$, Klf4- $\downarrow$, c-Myc- $\downarrow$ ) [115]. In addition, Tiemann et al. [116] tested 16 different stoichiometric ratios of the four factors and found that only seven combinations were successful in generating any reprogrammed colonies, suggesting that appropriate sustained levels of reprogramming factor expression are required to fully reprogram. Similarly, the length of reprogramming-factor expression is equally important. It has been shown that sustained expression for 12-16 days is needed for complete reprogramming of mouse fibroblasts to pluripotency [117]. This type of information may allow viral, episomal, and protein-based systems to be tailored to the reprogramming cell source type or for the addition of small molecules in order to increase the efficiency of one or more factors and thus achieve the optimal ratio.

\subsubsection{Target Cell Type}

Based on the transexpression of the prototypical reprogramming factors (OKSM), the importance of the type and origin of the cell to be reprogrammed cannot be overlooked. As with many scientific methods, there is a balance to be struck between ease of material 
procurement, target-cell reprogramming efficiency in relation to reprogramming method, species, and intended outcome or use of the reprogrammed cell line. Keratinocytes and peripheral blood cell types, such as T cells, are readily available and reasonably receptive to reprogramming irrespective of reprogramming method. Fibroblasts are equally easy to acquire, yet are as much as $100 \times$ less efficient in yielding iPSC lines than keratinocytes using a viral OKSM methodology [118]. The availability of cord blood as a reprogramming source material has the added advantage of potentially only requiring Oct 4 and Sox 2 to reprogram to iPSC, due in part to the relatively high proportion of proliferative progenitor phenotypes found in cord blood [119]. For some disease-specific iPSC derivation strategies, blood samples may be the most readily available source material. However, numerous biobanks are beginning to archive and make available samples from well-characterized familial disease trees based on skin fibroblast lines in order to aid investigators. It is still not entirely clear whether a strict hierarchy can be assigned to any given cell type or reprogramming method with respect to overall efficiency. Alternatively, it may be more helpful to categorize such potential hierarchies in the context of the methods and cell sources available, as well as the intended use of the derived reprogramming products. The potential use as a therapeutic will require significantly different methods than iPSC lines derived for research use, so some situational flexibility will play a role in the final choice of cell sourcing and reprogramming method. Balancing the availability of the cell source with the potential efficiency of reprogramming is not insignificant. Generally speaking, highly specialized terminally differentiated cell types like $\mathrm{T}$ lymphocytes and fibroblasts are easy to obtain yet harder to reprogram, while cord blood cells and germline cells are the reverse. While these gross generalizations may be true on the surface, new refinements and à-la-carte strategies for reprogramming may change these beliefs to some extent.

\subsection{Reprogramming Methods}

Early methods have utilized viral vectors which integrate into the genome to boost the efficiency of reprogramming to pluripotency. However, the potential for insertional mutagenesis and aberrant expression of reprogramming genes embedded in the genome impedes the path of reprogrammed cells to the clinic. So, recent efforts have turned to the development of nonintegrative reprogramming methods, including nonviral episomal plasmid systems, non-DNA methods, and nonintegrative viral systems with or without reprogramming enhancers [120-124].

\subsubsection{Viral-Driven}

The convenience and effectiveness of viral-based reprogramming methods makes them a valuable methodology for preparing iPSCs from numerous cell types from a number of species. While the lack of a "footprint"-free resultant cell line is currently problematic for downstream translational applications of these cells, the method itself, especially with the later iterations that are now widely used, make viral delivery of reprogramming factors for research-directed cell lines a viable and successful approach. Having said that, new nonintegrative viral systems such as adeno-associated virus- or sendai virus-based delivery systems are becoming more popular and replacing the traditional retroviral and lentiviral 
gene-delivery systems. However, the choice of delivery system is primarily dependent upon the target cell type and species, as well as the intended use of the reprogrammed products. The original retroviral-based reprogramming studies showed both success and ample room for improvement [29]. This work has been confirmed and extended by other groups, which showed that stable genomic integration and high expression of four factors - Oct4/Sox $2 / \mathrm{Klf} 4 / \mathrm{c}-\mathrm{Myc}$ or Oct4/Sox $/ \mathrm{Nanog} / \mathrm{Lin} 28$ - can reprogram fibroblast cells, B cells, and liver and stomach epithelial cells into iPSCs [30, 125]. The main drawback to the integrative viral methods is the unpredictable and variable nature of the genomic integration of the reprogramming genes, especially when one or more of these factors are known oncogenes. Several genetic-engineering advances have sought to minimize these unforeseen effects through polycistronic single-vector delivery systems and the use of Cre-loxP-expression systems [126, 127]. While these systems allow for more controlled integration and virtually complete excision of the reprogramming factors, respectively, neither completely resolves the potential for unforeseen insertional or mutational affects to the host genome.

Using nonintegrating adenovirus or sendai virus delivery systems, successful four-factor reprogramming has been performed on human and mouse cells with a variety of cell types $[121,122]$. These transient expression systems utilize host machinery, both cytoplasmic and nuclear, without integrating into the host genome and, like episomal transfection-based methods, the absence of specific vector sequences can be verified in fully reprogrammed cell lines through standard PCR methods. This is becoming an increasingly sought after quality with respect to the iPSC derivation process as a whole. Adenovirus and adeno-associated viral systems are relatively less efficient than integrating and sendai-based viral delivery systems, but offer increased safety characteristics [128]. The combined bump in efficiency and nonintegrative qualities for the sendai virus delivery system makes it an increasingly popular alternative to standard viral methods [121]. One drawback of this methodology is the current cost of the delivery system, putting large-panel derivations out of the reach of most investigators.

Other viral vector systems, such as lentivirus and gamma-retrovirus, require integration into the host genome for replication and can therefore disrupt the genomic integrity of the cells that are reprogrammed, rendering iPSCs and their derivatives less safe for clinical applications or altering gene-expression patterns, which can compromise compound screens or disease pathway analyses. Overall, the classic lentiviral or retroviral systems and more sophisticated constructs allowing selective removal of viral sequences still represent one of the most efficient and economical methodologies for reprogramming somatic cells of all types. Clearly, the integrating viral delivery methods can be upwards of $1000 \times$ more efficient at generating fully reprogrammed cell lines (Table 8.2), but these and other factors must be weighed when choosing a reprogramming methodology. It has been shown that residual expression of reprogramming factors can affect the transcriptional machinery and epigenetic profile of reprogrammed cells and should be factored into the overall cell-line development strategy [140-142].

\subsubsection{Nucleic Acid/Episomal-Driven}

An economical and yet relatively inefficient choice for somatic cell reprogramming is the use of nucleic acid in either linearized-DNA or episomal-vector formats $[120,132$, 


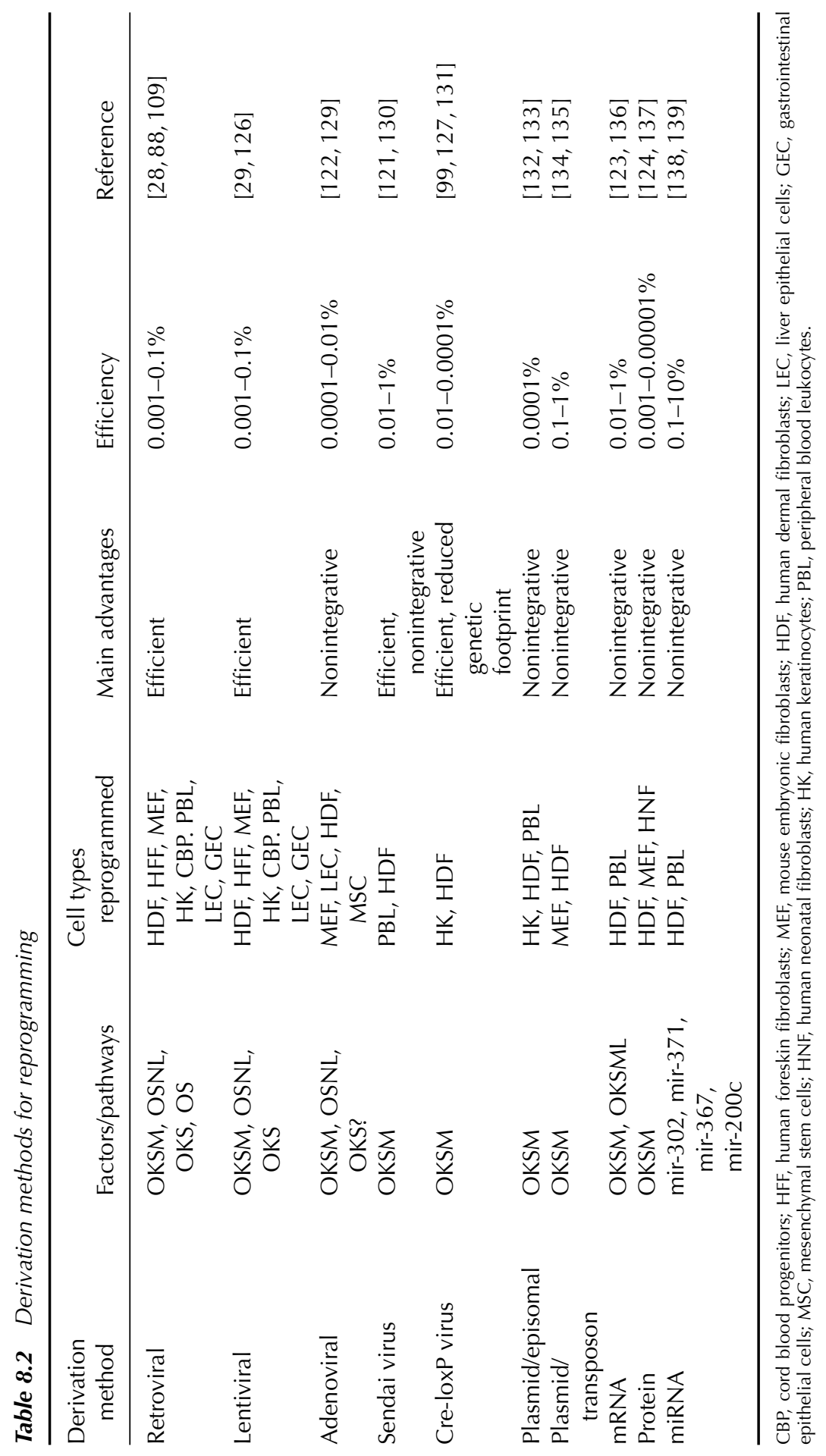


133, 143]. This method allows for the generation of iPSC lines that are free of vector or transgene sequences and have been used to reprogram a variety of somatic cell types. More recently, episomal reprogramming has been used in conjunction with a cocktail of small molecules to increase efficiencies 6-10-fold over episomal vector alone [144]. Serial transfection of multiple or single polycistronic plasmids is required for even modestly efficient reprogramming for most vector systems. Yu et al. [144] designed an EpsteinBarr virus nuclear antigen EBNA-containing episome that is self-replicating, obviating the need for multiple transfections and certainly reducing cell loss due to transfection toxicity. However, this nonintegrative episomal delivery system does not guarantee complete lack of plasmid integration and may require extensive dilution through cell replication to remove all instances of plasmid sequences [145]. Several important parameters govern success in episomal reprogramming, including but not limited to: (i) transfection method and efficiency; (ii) rapid dilution of transfected vector due to highly proliferative target cells; (iii) active silencing of plasmid sequences through host methylation; (iv) target cell type; and (v) the overall size of the minicircle vector [128]. In all episomal reprogramming methods, proper post-reprogramming screening should include molecular methods for the detection of vector sequences. Episomal delivery offers significant advantages over other methods, including an increased safety profile and compatibility with any number of external factors/enhancers that enable the tailoring of the method to research or therapeutic development requirements.

\subsection{3 mRNA-Driven}

A significant shift in nucleic acid-based reprogramming came in 2010 when Rossi and colleagues published on the highly efficient use of synthetic mRNA species to orchestrate reprogramming in multiple human cell types [123]. This method of using synthetic mRNA species to drive exogenous expression of the four canonic Yamanaka reprogramming factors proved to be a reliable (up to $10000 \times$ more efficient than standard episomal reprograming systems; see Table 8.2) defined method that gives rise to footprint-free iPSC lines. One drawback is the repeated transfections required over 12-16 days to ensure sufficiently stable factor expression to give rise to completely reprogrammed cell lines. Some recent effort has been devoted to qualitative changes in mRNA design, delivery, and screening methods that can reduce this production time, but the method still requires daily transfections for an extended period of time [146]. It is suggested that the initial investment in reagents and effort with the mRNA method can be recouped in the high frequency of fully reprogrammed colonies resulting from the method, thereby saving on screening time. As reprogramming methods push toward solutions enabling clinical use of iPSC lines derived from patients as personalized medicine, it is clear that nonintegrative methods like mRNA are setting the pace.

\subsection{4 miRNA-Driven}

miRNAs could be as instrumental in reprogramming as the transcription factors or epigenetic regulators that are so well known as drivers of somatic cell reprogramming. miRNAs are known to be key regulators of cell fate in ESCs, the development of the blastocyst, and various differentiation pathways [147-151]. Therefore, it can easily be envisioned that miRNAs could be utilized to impart cell-fate decisions in somatic reprogramming through 
the activation and repression of lineage-specific genes. The use of miRNAs as adjuvants to reprogramming through the addition of selected miRNA species to episomal or viral vector systems may prove useful. The transcription factor OSK plus miR-291-3p, miR-294, or miR-295 has been shown to consistently increase the number of Oct4- GFP + colonies as compared to controls transduced with OSK alone. The miR-294 mimic showed the greatest effects, increasing efficiency by up to 10 -fold for transduced mouse embryonic fibroblasts (MEFs) [152]. Miyoshi et al. [138] showed that the transfection of a combination of mir-200c, mir-302s, and mir-369s could reprogram both mouse and human somatic cells. It has recently been shown that the mechanism by which sodium butyrate enhances hiPSC reprogramming efficiency works through the induction of the miR302/367 miRNA cluster [153]. The blending of traditional methodologies for reprogramming and miRNAs may prove a powerful combination and afford researchers selective and increasingly less invasive methods by which to generate iPSCs [154].

\subsubsection{Protein-Driven}

Protein-based delivery of reprogramming factors has been demonstrated, but it has extremely slow kinetics, requiring significant time investment - as much as 6 weeks [124]. Another drawback to the use of proteins as factor-delivery modules is that transmembrane delivery can be challenging and therefore intracellular localization is very inefficient [155]. Amino acid segments from HIV-TAT and other basic peptides (CPPs) can significantly increase membrane translocation and allow the delivery of nucleic acid or protein elements to the nucleus $[156,157]$. Improvements in the targeting or stability of engineered proteins may make these systems more effective in the future; however, current requirements for numerous applications of the reprogramming cocktail over many weeks hampers mainstream usage of such systems.

\subsubsection{External Factors/Enhancers}

Several studies have shown that epigenetic remodeling is a rate-limiting step in reprogramming [109, 153, 158]. As shown in Table 8.3, many of the compounds that have a positive effect on reprogramming efficiency are involved in DNA methylation and chromatin remodeling. While this list is by no means exhaustive, it gives a broad sense of the pathways and fundamental approaches being taken to improve cellular reprogramming methods. By using one or more combinations of small molecules at the appropriate times during the reprogramming process, one may be able to avoid, at least partially, the permanent genome modifications engendered by the application of exogenous oncogene products for extended periods. These compounds can be roughly divided into three categories: (i) cell-cycle and metabolic regulators; (ii) epithelial-mesenchymal transition (EMT) phenotypic modulators; and (iii) epigenetic modifiers. Depending on the target cell source, a single factor or a defined cocktail can provide significant reprogramming enhancement by altering the kinetics or aiding in the progression through transitional phenotypes [159-162]. It is unclear what effects, if any, these pathway-specific or genetic modifiers might have on the genetic stability of the cell lines being generated, so exposure should be targeted and should be as brief as possible. Risk should obviously be weighed based on several criteria, not the least of which would be limiting the time of exposure to the reprogramming factors and the time spent transitioning through epigenetic remodeling. 


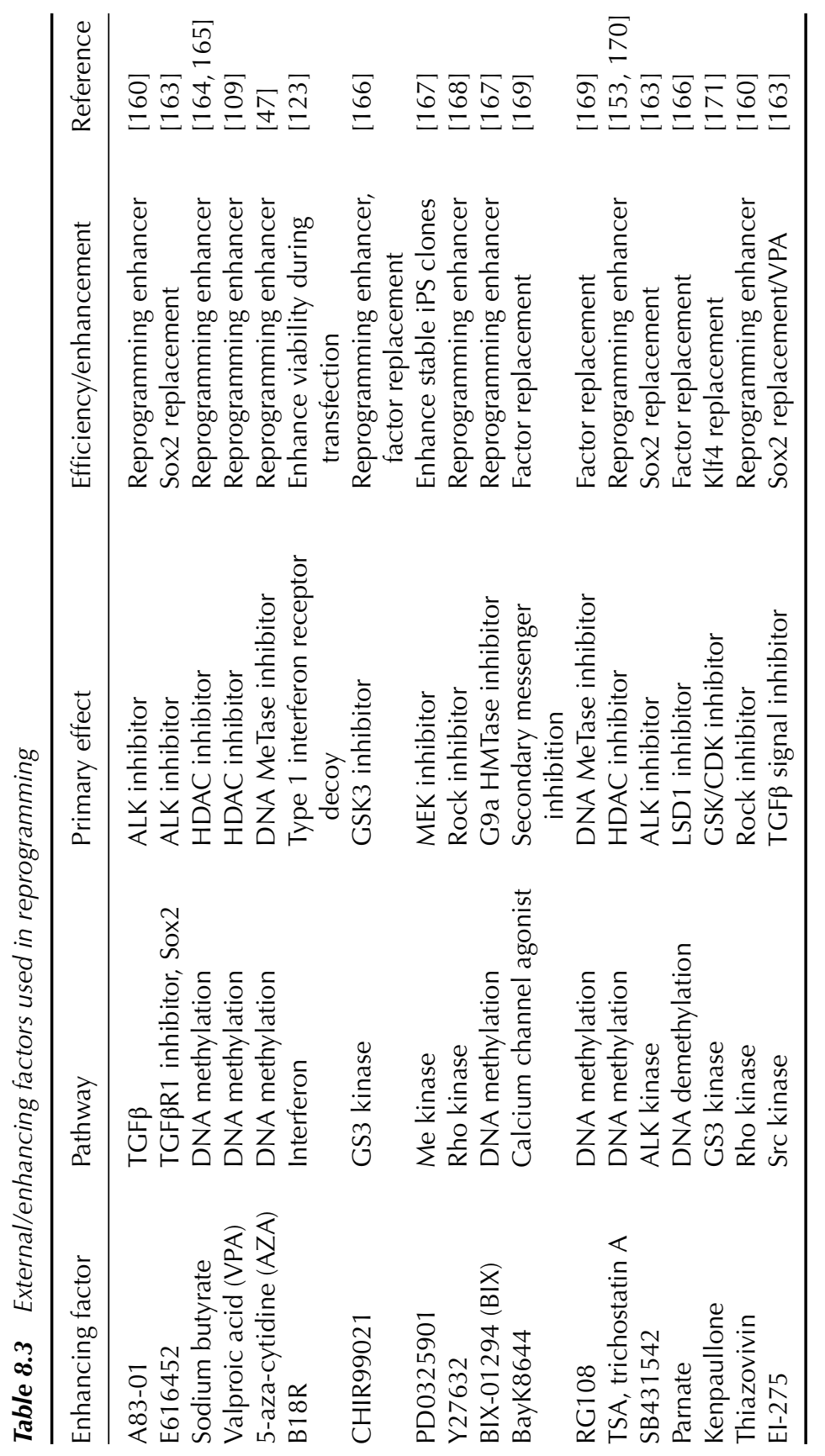




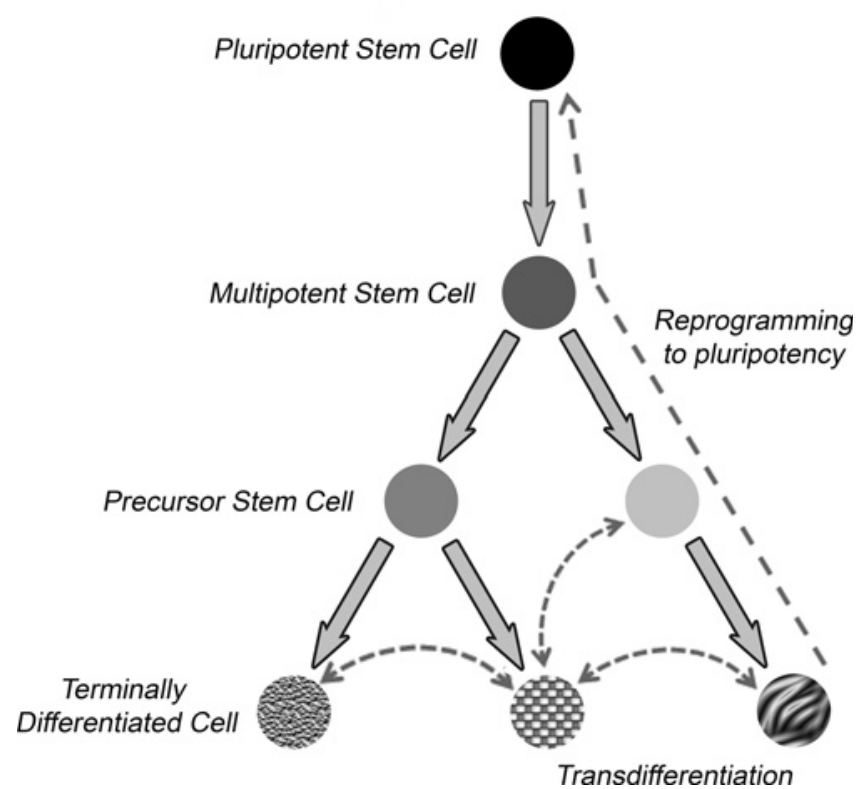

Figure 8.3 Cellular reprogramming

\subsubsection{Direct Reprogramming}

One potentially transformative advance in cellular reprogramming is the possibility of bypassing the pluripotent stem-cell intermediate and going right to the differentiated cell type of interest through a process termed "transdifferentiation" or lineage conversion. In the 1950s, Conrad Waddington described development by imagining an "epigenetic landscape" in which cells developed along specific pathways and through defined developmental states [172], similar to the branched structure seen in Figure 8.3. While this model remains an elegant means of describing development, Waddington likely did not envision the plasticity of cell states, even in terminally differentiated cells, that allows cells to be reverted to pluripotency via reprogramming. However, processes that are even more radical have emerged whereby differentiated cells can be directly converted from one type to another without passing through pluripotency (Figure 8.3). This direct conversion, or transdifferentiation, allows for an abundant and reproducible adult cell source to be directly converted to the regenerative cell type of interest, whether for research or therapeutic application $[4,173]$. The first demonstration of transdifferentiation was made in 1986 when researchers in the laboratory of Harold Weintraub showed that expression of a single transfected gene, MyoD, could convert fibroblasts to myoblasts [174]. More recently, several groups have made remarkable progress in this offshoot of reprogramming technology. In 2008, Zhou et al. [175] showed that the overexpression of Ngn3, Pdx1, and Mafa in mouse pancreatic exocrine cells induced them to convert into insulin-producing endocrine cells. Even more remarkably, this conversion was done in vivo, providing a distinct advantage to therapeutic applications of this technology. This was followed by demonstrations by several groups 
of transdifferentiation of fibroblasts into a variety of other somatic cell types representing different germ layers, including neurons, hepatocytes, and cardiomyocytes [176-178], as well as precursor cells in different lineages, such as hematopoietic progenitors [179]. In addition, it was shown recently that direct reprogramming can be accomplished, including the transdifferentiation of fibroblasts to myocytes and neuronal cells using nonviral and nonintegrative methods [180-182]. The rapidity and relatively high efficiency of these transdifferentiation protocols seems to indicate a preferred path for reprogramming technology, especially since they lack potentially oncogenic reprogramming factor-induced intermediates, making them safer for clinical applications.

\subsection{Applications and Future Trends}

Reprogramming technology has the potential to significantly impact a broad spectrum of biomedical research, from basic studies through clinical applications. As a tool for basic research, ESCs and iPSCs are already making significant contributions, which will serve as the foundation for the development of clinical uses. Studies using ESCs and iPSCs are helping to elucidate the molecular and cellular mechanisms controlling development from the early embryo to specific cell lineages [183]. As our understanding of the differentiation process evolves, the complex interactions of cell signaling, transcription factors, and epigenetic changes necessary for development are becoming clearer. To recapitulate the differentiation process in vitro, cells must often be painstakingly guided through numerous and precise intermediate cell types in order to form fully functional, terminally differentiated cells (as exemplified by the production of pancreatic $\beta$-cells [184]). Such studies can be hampered by variability in the differentiation capacity of different pluripotent stem-cell lines [33, 185], which is even more pronounced in iPSCs due to epigenetic memory as well as the genetic and epigenetic changes that occur during reprogramming and culture [186-189].

In addition to the study of normal development, cellular reprogramming technology is being used to create new model systems to study the pathology of many different diseases. Currently, reprogrammed stem-cell models have been developed for over 80 different genetic diseases (Table 8.4) [190-192]. These cell lines, coupled with ever-improving differentiation protocols, allow researchers to derive large quantities of specific target cell types affected in a disease and, importantly, to study progression of the disease through development. Disease-specific stem-cell models are of great value in high-throughput drug screening and toxicity assessment [193]. The value of these iPSC models is such that several derivation and banking initiatives are either being discussed or are underway, including a large-scale collaboration between academic institutions and pharmaceutical companies in Europe [194] and an initiative in the United States funded by the California Institute for Regenerative Medicine (CIRM).

\subsubsection{Moving Toward Clinical Applications for Cellular Reprogramming}

Researchers have long recognized the potential for the development of "patient-specific" pluripotent stem cells in the treatment of disease [262]. Shortly after the development of 
Table 8.4 Reprogrammed disease models

\begin{tabular}{|c|c|c|}
\hline Disorder & Affected gene(s) & Reference \\
\hline$\alpha$-1-antitrypsin deficiency & $\begin{array}{l}\text { SERPINA1 ( } \alpha 1 \text {-antitrypsin) gene } \\
\text { mutation (homozygous) }\end{array}$ & [195] \\
\hline Alzheimer's disease (familial) & PS1 and PS2 gene mutations & [196] \\
\hline Alzheimer's disease (familial) & APP gene duplication & [197] \\
\hline Alzheimer's disease (sporadic) & $?$ & [197] \\
\hline Amyotrophic lateral sclerosis & SOD1 gene mutation & [198] \\
\hline Amyotrophic lateral sclerosis & TARDBP gene mutation & [199] \\
\hline $\begin{array}{l}\text { Amyotrophic lateral sclerosis } \\
\text { (ALS8) }\end{array}$ & VAPB gene mutation & {$[200]$} \\
\hline Angelman syndrome & $\begin{array}{l}\text { Maternally inherited deletion of } \\
\text { chromosome } 15 q^{11}-q^{13}\end{array}$ & [201] \\
\hline $\begin{array}{l}\text { Arrhythmogenic right-ventricular } \\
\text { cardiomyopathy }\end{array}$ & PKP2 gene mutation & {$[202]$} \\
\hline Ataxia-telangiectasia & ATM gene mutation & [203] \\
\hline Atypical Werner syndrome & LMNA gene mutation & {$[204]$} \\
\hline Becker muscular dystrophy & DMD gene, unidentified mutation & [205] \\
\hline Best disease & BEST1 gene mutation & [206] \\
\hline$\beta$-thalassaemia & HBB gene mutation & [207] \\
\hline Cartilage-hair hypoplasia & RMRP gene mutation & [208] \\
\hline $\begin{array}{l}\text { Catecholaminergic polymorphic } \\
\text { ventricular tachycardia } \\
\text { (dominant) }\end{array}$ & RYR2 gene mutation & [209] \\
\hline $\begin{array}{l}\text { Catecholaminergic polymorphic } \\
\text { ventricular tachycardia } \\
\text { (recessive) }\end{array}$ & $\begin{array}{l}\text { CASQ2 gene mutation } \\
\text { (homozygous) }\end{array}$ & [210] \\
\hline $\begin{array}{l}\text { Chronic infantile neurologic } \\
\text { cutaneous and articular } \\
\text { syndrome }\end{array}$ & NLRP3 gene mutation & [211] \\
\hline Cockayne syndrome & ERCC6 gene mutation & [212] \\
\hline Crigler-Najjar syndrome & Deletion in UGT1A1 gene & [213] \\
\hline Cystic fibrosis & $\begin{array}{l}\text { CFTR gene mutation, } \\
\text { homozygous }\end{array}$ & [195] \\
\hline Diabetes, type 1 & Multifactorial & {$[214]$} \\
\hline Diabetes, type 2 & Multifactorial & {$[215]$} \\
\hline Dilated cardiomyopathy & TNNT2 gene mutation & [216] \\
\hline Dilated cardiomyopathy & LMNA gene mutation & [204] \\
\hline Down syndrome & Trisomy of chromosome 21 & [217] \\
\hline Duchenne muscular dystrophy & $\begin{array}{l}\text { DMD gene (deletion of exons } \\
45-52 \text { ) }\end{array}$ & [205] \\
\hline Dyskeratosis congenita & Deletion in DKC1 gene & {$[218]$} \\
\hline Familial dysautonomia & $I K B K A P$ gene mutation & [219] \\
\hline Familial hypercholesterolemia & LDLR gene mutation & [220] \\
\hline $\begin{array}{l}\text { Familial hypertrophic } \\
\text { cardiomyopathy }\end{array}$ & MYH7 gene mutation & [221] \\
\hline Fanconi's anemia & $\begin{array}{l}\text { FANCA and FANCD2 gene } \\
\text { mutations }\end{array}$ & [222] \\
\hline
\end{tabular}


Table 8.4 (Continued)

\begin{tabular}{|c|c|c|}
\hline Disorder & Affected gene(s) & Reference \\
\hline Facioscapulohumeral dystrophy & $\begin{array}{l}\text { Deletion of } 4 \mathrm{q} \text { D4Z4 } \\
\text { microsatellite repeats }\end{array}$ & {$[223]$} \\
\hline Fragile $X$ syndrome & $\begin{array}{l}\text { FMR1 gene CGG triplet repeat } \\
\text { expansion }\end{array}$ & {$[224]$} \\
\hline Friedreich's ataxia & $\begin{array}{l}\text { FXN gene GAA triplet repeat } \\
\text { expansion }\end{array}$ & [225] \\
\hline Gaucher disease, types 1, 2, 3 & GBA gene mutations & {$[226]$} \\
\hline Glycogen storage disease, type $1 \mathrm{a}$ & G6PC gene mutation & {$[220]$} \\
\hline Glycogen storage disease, type $1 \mathrm{~b}$ & SLC37A gene mutation & {$[213]$} \\
\hline Herpes simplex encephalitis & STAT1 or TLR3 gene mutation & [208] \\
\hline Huntington disease & $\begin{array}{l}\text { HTT gene CAG triplet repeat } \\
\text { expansion }\end{array}$ & [227] \\
\hline $\begin{array}{l}\text { Hutchinson-Gilford progeria } \\
\text { syndrome }\end{array}$ & LMNA gene mutation & {$[228]$} \\
\hline LEOPARD syndrome & PTPN11 mutation (heterozygous) & [229] \\
\hline Lesch-Nyhan syndrome & $\begin{array}{l}\text { HPRT1 gene mutation } \\
\text { (homozygous) }\end{array}$ & {$[230]$} \\
\hline $\begin{array}{l}\text { Limb-Girdle muscular dystrophy } \\
\text { 2D }\end{array}$ & SGCA gene mutation & [231] \\
\hline Long QT syndrome, type 1 & KCNQ1 gene mutation & {$[232]$} \\
\hline Long QT syndrome, type 2 & $\mathrm{KCNH} 2$ gene mutation & {$[233]$} \\
\hline $\begin{array}{l}\text { Long QT syndrome, type } 3 \\
\text { (Brugada syndrome) }\end{array}$ & SCN5A gene mutation & {$[234]$} \\
\hline $\begin{array}{l}\text { Machado-Joseph disease } \\
\quad \text { (spinocerebellar ataxia type } 3)\end{array}$ & $\begin{array}{l}\text { MJD1 (ATXN3) gene CAG triplet } \\
\text { repeat expansion }\end{array}$ & [235] \\
\hline Marfan syndrome & FBN1 gene mutation & {$[236]$} \\
\hline $\begin{array}{l}\text { Mitochondrial } \\
\text { encephalomyopathy with lactic } \\
\text { acidosis and stroke-like } \\
\text { episodes (MELAS) }\end{array}$ & Mitochondrial gene mutations & {$[237]$} \\
\hline $\begin{array}{l}\text { Mucopolysaccharidosis, type } 1 \\
\text { (Hurler syndrome) }\end{array}$ & IDUA gene & {$[238]$} \\
\hline Mucopolysaccharidosis, type IIIB & $\begin{array}{l}\text { NAGLU gene mutation } \\
\text { (homozygous) }\end{array}$ & [239] \\
\hline $\begin{array}{l}\text { Myeloproliferative disorder } \\
\text { (polycythemia vera) }\end{array}$ & JAK2 gene mutation & {$[240]$} \\
\hline $\begin{array}{l}\text { Myeloproliferative disorder } \\
\text { (primary myelofibrosis) }\end{array}$ & JAK2 gene mutation & [240] \\
\hline Omenn syndrome & RAG1 gene mutation & {$[208]$} \\
\hline Osteogenesis imperfecta & COL1A2 gene mutation & {$[241]$} \\
\hline Parkinson's disease & Multifactorial & [131] \\
\hline $\begin{array}{l}\text { Parkinson's disease, autosomal } \\
\text { recessive, young onset }\end{array}$ & PARK2 gene mutation & {$[242]$} \\
\hline Parkinson's disease, familial & PINK1 and LRRK2 gene mutations & [243] \\
\hline
\end{tabular}


Table 8.4 (Continued)

\begin{tabular}{|c|c|c|}
\hline Disorder & Affected gene(s) & Reference \\
\hline Pelizaeus-Merzbacher disease & PLP1 gene, partial duplication & [244] \\
\hline Pompe disease & GAA gene mutation & {$[245]$} \\
\hline Prader-Willi syndrome & $\begin{array}{l}\text { Paternal deletion of chromosome } \\
15 q^{11}-q_{13}\end{array}$ & {$[201]$} \\
\hline $\begin{array}{l}\text { Progressive familial hereditary } \\
\text { cholestasis }\end{array}$ & Multifactorial & [213] \\
\hline $\begin{array}{l}\text { Recessive dystrophic } \\
\text { epidermolysis bullosa }\end{array}$ & COL7A1 gene mutation & [246] \\
\hline Retinitis pigmentosa & $\begin{array}{l}\text { Mutations in RP9, RP1, PRPH2 or } \\
\text { RHO genes }\end{array}$ & {$[247]$} \\
\hline Rett syndrome & MECP2 gene mutation & {$[248]$} \\
\hline Rett syndrome (Hanefeld variant) & CDKL5 gene mutation & [249] \\
\hline Sandhoff disease (mouse model) & $\begin{array}{l}\text { HexB gene deletion } \\
\text { (homozygous) }\end{array}$ & [250] \\
\hline Severe congenital neutropenia & ELANE gene mutation & {$[251]$} \\
\hline Schizophrenia & $\begin{array}{l}\text { Complex trait, DISC1 gene } \\
\text { mutation }\end{array}$ & [252] \\
\hline Scleroderma & $?$ & [195] \\
\hline $\begin{array}{l}\text { Severe combined } \\
\text { immunodeficiency (ADA-SCID) }\end{array}$ & ADA gene mutation & {$[205]$} \\
\hline $\begin{array}{l}\text { Severe combined } \\
\text { immunodeficiency syndrome } \\
\text { (SCID) }\end{array}$ & RAG1 gene mutation & [208] \\
\hline Sickle-cell anemia & $\begin{array}{l}\text { HBB gene mutation } \\
\text { (homozygous) }\end{array}$ & [195] \\
\hline Spinal muscular atrophy, type 1 & $\begin{array}{l}\text { SMN1 gene mutation } \\
\text { (homozygous) }\end{array}$ & [253] \\
\hline Spinocerebellar ataxia, type 2 & $\begin{array}{l}\text { ATXN2 gene CAG triplet repeat } \\
\text { expansion }\end{array}$ & {$[254]$} \\
\hline $\begin{array}{l}\text { Shwachman-Bodian-Diamond } \\
\text { syndrome }\end{array}$ & $\begin{array}{l}\text { SBDS gene mutation, } \\
\text { multifactorial }\end{array}$ & [205] \\
\hline Supravalvular aortic stenosis & ELN gene mutation & {$[255]$} \\
\hline Systemic lupus erythematosus & $?$ & {$[256]$} \\
\hline Timothy syndrome & CACNA1C gene mutation & [257] \\
\hline Tyrosinemia, type 1 & FAH gene mutation & [213] \\
\hline Williams-Beuren syndrome & ELN gene microdeletion & [258] \\
\hline Wilson's disease & ATP7B gene mutation & [259] \\
\hline $\begin{array}{l}\text { X-linked adrenoleukodystrophy, } \\
\text { adrenomyeloneuropathy (AMN) }\end{array}$ & $\mathrm{ABCD} 1$ gene mutation & {$[260]$} \\
\hline $\begin{array}{l}\text { X-linked adrenoleukodystrophy, } \\
\text { childhood cerebral ALD } \\
\text { (CCALD) }\end{array}$ & ABCD1 gene mutation & {$[260]$} \\
\hline $\begin{array}{l}\text { X-linked chronic granulomatous } \\
\text { disease }\end{array}$ & CYBB gene mutation & {$[261]$} \\
\hline
\end{tabular}


cellular reprogramming, the "proof of principle" for the use of this technology was elegantly demonstrated by Rudolf Jaenisch and coworkers using a mouse model for sickle-cell anemia [263]. In this study, fibroblasts for the sickle-cell mouse were reprogrammed to a pluripotent state. These iPSCs were then modified by gene targeting to correct the mutated hemoglobin allele, and the "corrected" iPSCs were differentiated into hematopoietic progenitor cells. The mouse was then cured by transplantation of these corrected autologous progenitors. While this established the roadmap to therapeutic application of reprogramming technology, numerous obstacles remained. One of the primary issues surrounding the use of any pluripotent stem cells in humans is the risk of tumorigenesis, since pluripotent cells are capable of unlimited self-renewal. To mitigate this risk, cells introduced into a patient would need to be a pure population of differentiated cells. The processing steps required to achieve such purity specifications make clinical cell manufacturing with iPSCs or ESCs particularly challenging. In addition, the tumorigenicity risk is likely even higher for iPSCs than for ESCs, due in part to the accumulation of epigenetic and genetic abnormalities during reprogramming, which could promote tumor formation [264]. Thus, further study of the reprogramming process and development of new reprogramming methods will likely be required before the clinical application of iPSC technology is fully realized.

Despite the risks, the movement of pluripotent stem cells toward the clinic is proceeding. There is currently a phase I clinical trial in the United States for the use of hESC-derived retinal pigment epithelial cells for the treatment of macular degeneration [265, 266]. Furthermore, although there are still many unknowns regarding the use of iPSCs in the clinic, a similar trial using reprogrammed cells is underway in Japan [267].

\subsubsection{The Merging of Stem Cells and New Methods of Genetic Engineering}

To achieve the overall goal of using cellular reprogramming to treat genetic diseases, methods of correcting the relevant mutations must be developed. Although homologous recombination has been used for many years to modify the genome of mouse cells (including ESCs) [268], the process is very inefficient in human cells, hampering its utility. However, recent advances in the use of zinc finger nucleases (ZFNs) and, more recently, transcription activator-like effector nucleases (TALENs) and the RNA-guided CRISPR (clustered regularly interspaced short palindromic repeats)/Cas system, are providing new tools for human gene modification. These technologies allow the production of nucleases that target specific sequences in the genome and have been successfully used in gene modification of hESCs and iPSCs [269-271]. TALENs have also been used to correct the genetic defect in disease-specific iPSCs [272], moving one step closer to realizing stem-cell therapy in the treatment of genetic disorders. Interestingly, TALENs have also been used to introduce specific disease-causing mutations into normal iPSCs, providing a new way of generating disease models with iPSCs [273].

\subsubsection{Efficiency, Expense, and Safety}

Although great strides have been made in the development of reprogramming-method alternatives since the original report in 2006, there are still significant hurdles to overcome in order to make somatic-cell reprogramming a reliable research and diagnostic tool and a 
viable therapeutic option. As has been suggested by Gonzalez and others, the current à-lacarte reprogramming menu does and will allow purposeful innovation in the field, but it also introduces the problem of having so many variations on the theme that method adoption and standardization remain considerable challenges [128]. Significant progress has also been made toward developing safer and more efficient methods of reprogramming. However, a balance must be sought for the three-legged stool, which should include cost [121-123, 274]. As always, consideration of the intended use for the cell lines being developed is a main driver in the choice of reprogramming method, and as standards for line safety and basic characterization parameters are established these choices may narrow. Cost, as a function of the reagents and the investigator time required to perform reprogramming protocols and to fully mature and characterize the resultant cell lines, is also of interest. Many of the safer viral systems and the numerous potential enhancing reagents are currently driving expenditures per source derivation over $\$ 5000$, including personnel effort and rudimentary characterization screening. While the cost of other methods can be considerably less, one can see how the development of panels of cell lines for drug screening or disease modeling may be prohibitively expensive. For these and other reasons, it is difficult to find a onesize-fits-all reprogramming strategy. In addition, increased safety and enhanced efficiency do not always translate into decreased variation within the derived panel of cell lines [275]. This fact demands the preparation and at least partial characterization of multiple lines for a single tissue source to ensure availability of cell lines with qualified genetic stability and pluripotency, thus exacerbating the cost problem.

\subsubsection{Developing Standards}

Beginning from as early as 1998, when Thomson and colleagues published the derivation of human ESCs, a significant body of work has been devoted to creating a set of standards for the development, culture, and characterization of this powerful stem cell resource [16, $276,277]$. However, since the introduction of iPSC derivation in 2006, much of the effort of the field has been focused on refining the various methods and investigating the boundaries of their potential uses. As this field moves forward, it will be essential to be able to assess the various methodological enhancements using generally, if not universally, accepted standards of phenotype and function. Proving complete reprogramming in human cells is not trivial, and it may not be possible to show definitively to the satisfaction of all for some time. While several groups have begun to venture down this path, setting convention in a time of unprecedented growth for the field is a daunting task. Clearly detailed genomic, transcriptional, and functional evaluations make up a partial list of broad analysis groups that can be entertained as potential characterization standards. However, in a broader sense, the development of standards around tissue sourcing, screening mechanisms, data reporting, confidentiality, and banking should be a companion discussion to the former detailed individual analysis. Screening tools, robotics, and selection methods need to be developed to aid the discrimination and selection of fully reprogrammed cells and those that harbor potential defects that would affect their performance as research tools or as therapeutics. The method by which this is done is still a matter of some debate and still needs to be developed for human cell analysis [34, 278, 279]. However, previous work with ESC characterization could serve as a rough template for the development of iPSC standards. 


\subsection{Conclusion}

Our understanding of the role of stem cells in development has advanced significantly over the past 50 years. Dissection of the complex interplay between genes, proteins, and RNAs that maintain pluripotency and drive differentiation has spurred the development of revolutionary new cellular reprogramming technologies. The ability to reprogram cells to pluripotency and to "transdifferentiate" cells from one type to another emphasizes the remarkable plasticity of our cells and has opened up tantalizing new opportunities in the study of disease and in regenerative medicine. However, much remains to be done. We are still uncovering the unique properties of iPSCs and new methods for their production and differentiation using an array of viral, RNA, protein, and chemical tools that continue to be developed. Improvements in the efficiency and safety of reprogramming and differentiation protocols are critical, and considerations of an administrative nature, including the development of policies and standards, should also be taken into account as the field moves closer to the ultimate goal of clinical application.

These landmark studies have uncovered the potential of stem cells, demonstrated cellular plasticity, and shown our increasing ability to manipulate cell identities.

The core pluripotency regulators Oct4, Nanog, and Sox 2 control a network of proteincoding genes, miRNAs, and lincRNAs in order to regulate pluripotency. These three factors autoregulate their expression and upregulate expression of the genes associated with pluripotency. In turn, these pluripotency proteins and RNAs interact with the core factors and the polycomb complexes PRC1 and PRC2 to repress expression of lineage-specific proteins and RNAs. When activated, the lineage-specific factors repress the polycomb complexes and downregulate expression of the core pluripotency factors.

Where most reprogramming techniques take the differentiated cell back to pluripotency, direct conversion or transdifferentiation involves switching from one differentiated cell type to another without conversion to a pluripotent state.

\section{References}

(1) Gurdon, J.B., Elsdale, T.R., and Fischberg, M. (1958) Sexually mature individuals of Xenopus laevis from the transplantation of single somatic nuclei. Nature, 182 (4627), 64-65.

(2) Till, J.E. and McCulloch, E.A. (1961) A direct measurement of the radiation sensitivity of normal mouse bone marrow cells. Radiation Research, 14, 213-222.

(3) Stadtfeld, M. and Hochedlinger, K. (2010) Induced pluripotency: history, mechanisms, and applications. Genes \& Development, 24 (20), 2239-2263.

(4) Vierbuchen, T. and Wernig, M. (2011) Direct lineage conversions: unnatural but useful? Nature Biotechnology, 29 (10), 892-907.

(5) Van de Velde, H., Cauffman, G., Tournaye, H. et al. (2008) The four blastomeres of a 4-cell stage human embryo are able to develop individually into blastocysts with inner cell mass and trophectoderm. Human Reproduction, 23 (8), 1742-1747.

(6) Kleinsmith, L.J. and Pierce, G.B. (1964) Multipotentiality of single embryonal carcinoma cells. Cancer Research, 24, 1544-1551.

(7) Kahan, B.W. and Ephrussi, B. (1970) Developmental potentialities of clonal in vitro cultures of mouse testicular teratoma. Journal of the National Cancer Institute, 44 (5), 1015-1036.

(8) Hogan, B., Fellous, M., Avner, P., and Jacob, F. (1977) Isolation of a human teratoma cell line which expresses F9 antigen. Nature, 270 (5637), 515-518. 
(9) Fraley, E.E., Lange, P.H., Williams, R.D., and Ortlip, S.A. (1980) Staging of early nonseminomatous germ-cell testicular cancer. Cancer, 45 (Suppl. 7), 1762-1767.

(10) Przyborski, S.A., Christie, V.B., Hayman, M.W. et al. (2004) Human embryonal carcinoma stem cells: models of embryonic development in humans. Stem Cells and Development, 13 (4), 400-408.

(11) Martin, G.R. (1981) Isolation of a pluripotent cell line from early mouse embryos cultured in medium conditioned by teratocarcinoma stem cells. Proceedings of the National Academy of Sciences of the United States of America, 78 (12), 7634-7638.

(12) Evans, M.J. and Kaufman, M.H. (1981) Establishment in culture of pluripotential cells from mouse embryos. Nature, 292 (5819), 154-156.

(13) Bradley, A., Evans, M., Kaufman, M.H., and Robertson, E. (1984) Formation of germ-line chimaeras from embryo-derived teratocarcinoma cell lines. Nature, 309 (5965), 255-256.

(14) Nagy, A., Gocza, E., Diaz, E.M. et al. (1990) Embryonic stem cells alone are able to support fetal development in the mouse. Development (Cambridge, England), 110 (3), 815-821.

(15) Eggan, K., Akutsu, H., Loring, J. et al. (2001) Hybrid vigor, fetal overgrowth, and viability of mice derived by nuclear cloning and tetraploid embryo complementation. Proceedings of the National Academy of Sciences of the United States of America, 98 (11), 6209-6214.

(16) Thomson, J.A., Itskovitz-Eldor, J., Shapiro, S.S. et al. (1998) Embryonic stem cell lines derived from human blastocysts. Science, 282 (5391), 1145-1147.

(17) Itskovitz-Eldor, J., Schuldiner, M., Karsenti, D. et al. (2000) Differentiation of human embryonic stem cells into embryoid bodies compromising the three embryonic germ layers. Molecular Medicine, 6 (2), 88-95.

(18) Resnick, J.L., Bixler, L.S., Cheng, L., and Donovan, P.J. (1992) Long-term proliferation of mouse primordial germ cells in culture. Nature, 359 (6395), 550-551.

(19) Matsui, Y., Zsebo, K., and Hogan, B.L. (1992) Derivation of pluripotential embryonic stem cells from murine primordial germ cells in culture. Cell, 70 (5), 841-847.

(20) Shamblott, M.J., Axelman, J., Wang, S. et al. (1998) Derivation of pluripotent stem cells from cultured human primordial germ cells. Proceedings of the National Academy of Sciences of the United States of America, 95 (23), 13726-13731.

(21) Turnpenny, L., Brickwood, S., Spalluto, C.M. et al. (2003) Derivation of human embryonic germ cells: an alternative source of pluripotent stem cells. Stem Cells, 21 (5), 598-609.

(22) Pashai, N., Hao, H., All, A. et al. (2012) Genome-wide profiling of pluripotent cells reveals a unique molecular signature of human embryonic germ cells. PLoS One, 7 (6), e39088.

(23) Soza-Ried, J. and Fisher, A.G. (2012) Reprogramming somatic cells towards pluripotency by cellular fusion. Current Opinion in Genetics \& Development, 22 (5), 459-465.

(24) Miller, R.A. and Ruddle, F.H. (1976) Pluripotent teratocarcinoma-thymus somatic cell hybrids. Cell, 9 (1), 45-55.

(25) Cowan, C.A., Atienza, J., Melton, D.A., and Eggan, K. (2005) Nuclear reprogramming of somatic cells after fusion with human embryonic stem cells. Science, 309 (5739), 13691373.

(26) Wilmut, I., Schnieke, A.E., McWhir, J. et al. (1997) Viable offspring derived from fetal and adult mammalian cells. Nature, 385 (6619), 810-813.

(27) Tachibana, M., Amato, P., Sparman, M. et al. (2013) Human embryonic stem cells derived by somatic cell nuclear transfer. Cell, 153 (6), 1228-1238.

(28) Takahashi, K. and Yamanaka, S. (2006) Induction of pluripotent stem cells from mouse embryonic and adult fibroblast cultures by defined factors. Cell, 126 (4), 663-676.

(29) Takahashi, K., Tanabe, K., Ohnuki, M. et al. (2007) Induction of pluripotent stem cells from adult human fibroblasts by defined factors. Cell, 131 (5), 861-872.

(30) Yu, J., Vodyanik, M.A., Smuga-Otto, K. et al. (2007) Induced pluripotent stem cell lines derived from human somatic cells. Science, 318 (5858), 1917-1920.

(31) Smith, K.P., Luong, M.X., and Stein, G.S. (2009) Pluripotency: toward a gold standard for human ES and iPS cells. Journal of Cellular Physiology, 220 (1), 21-29.

(32) Burridge, P.W., Anderson, D., Priddle, H. et al. (2007) Improved human embryonic stem cell embryoid body homogeneity and cardiomyocyte differentiation from a novel V-96 plate aggregation system highlights interline variability. Stem Cells, 25 (4), 929-938. 
(33) Osafune, K., Caron, L., Borowiak, M. et al. (2008) Marked differences in differentiation propensity among human embryonic stem cell lines. Nature Biotechnology, 26 (3), 313-315.

(34) Bock, C., Kiskinis, E., Verstappen, G. et al. (2011) Reference maps of human ES and iPS cell variation enable high-throughput characterization of pluripotent cell lines. Cell, 144 (3), $439-452$.

(35) Stewart, M.H., Bosse, M., Chadwick, K. et al. (2006) Clonal isolation of hESCs reveals heterogeneity within the pluripotent stem cell compartment. Nature Methods, $\mathbf{3}$ (10), 807-815.

(36) Stewart, M.H., Bendall, S.C., Levadoux-Martin, M., and Bhatia, M. (2010) Clonal tracking of hESCs reveals differential contribution to functional assays. Nature Methods, 7 (11), 917-922.

(37) Silva, J. and Smith, A. (2008) Capturing pluripotency. Cell, 132 (4), 532-536.

(38) Nichols, J. and Smith, A. (2009) Naive and primed pluripotent states. Cell Stem Cell, 4 (6), 487-492.

(39) Brons, I.G., Smithers, L.E., Trotter, M.W. et al. (2007) Derivation of pluripotent epiblast stem cells from mammalian embryos. Nature, 448 (7150), 191-195.

(40) Tesar, P.J., Chenoweth, J.G., Brook, F.A. et al. (2007) New cell lines from mouse epiblast share defining features with human embryonic stem cells. Nature, 448 (7150), 196-199.

(41) Tachibana, M., Sparman, M., Ramsey, C. et al. (2012) Generation of chimeric rhesus monkeys. Cell 148 (1-2), 285-295.

(42) De Los Angeles, A., Loh, Y.H., Tesar, P.J., and Daley, G.Q. (2012) Accessing naive human pluripotency. Current Opinion in Genetics \& Development, 22 (3), 272-282.

(43) Najm, F.J., Chenoweth, J.G., Anderson, P.D. et al. (2011) Isolation of epiblast stem cells from preimplantation mouse embryos. Cell Stem Cell, 8 (3), 318-325.

(44) Chou, Y.F., Chen, H.H., Eijpe, M. et al. (2008) The growth factor environment defines distinct pluripotent ground states in novel blastocyst-derived stem cells. Cell, 135 (3), 449-461.

(45) Hanna, J., Cheng, A.W., Saha, K. et al. (2010) Human embryonic stem cells with biological and epigenetic characteristics similar to those of mouse ESCs. Proceedings of the National Academy of Sciences of the United States of America, 107 (20), 9222-9227.

(46) Gu, Q., Hao, J., Zhao, X.Y. et al. (2012) Rapid conversion of human ESCs into mouse ESC-like pluripotent state by optimizing culture conditions. Protein \& Cell, 3 (1), 71-79.

(47) Mikkelsen, T.S., Hanna, J., Zhang, X. et al. (2008) Dissecting direct reprogramming through integrative genomic analysis. Nature, 454 (7200), 49-55.

(48) Polejaeva, I. and Mitalipov, S. (2013) Stem cell potency and the ability to contribute to chimeric organisms. Reproduction (Cambridge, England), 145 (3), R81-88.

(49) Loh, Y.H., Yang, L., Yang, J.C. et al. (2011) Genomic approaches to deconstruct pluripotency. Annual Review of Genomics and Human Genetics, 12, 165-185.

(50) Young, R.A. (2011) Control of the embryonic stem cell state. Cell, 144 (6), 940-954.

(51) Boyer, L.A., Lee, T.I., Cole, M.F. et al. (2005) Core transcriptional regulatory circuitry in human embryonic stem cells. Cell, 122 (6), 947-956.

(52) Nichols, J., Zevnik, B., Anastassiadis, K. et al. (1998) Formation of pluripotent stem cells in the mammalian embryo depends on the POU transcription factor Oct4. Cell, 95 (3), 379-391.

(53) Ivanova, N., Dobrin, R., Lu, R. et al. (2006) Dissecting self-renewal in stem cells with RNA interference. Nature, 442 (7102), 533-538.

(54) Masui, S., Nakatake, Y., Toyooka, Y. et al. (2007) Pluripotency governed by Sox2 via regulation of Oct3/4 expression in mouse embryonic stem cells. Nature Cell Biology, 9 (6), 625-635.

(55) Chew, J.L., Loh, Y.H., and Zhang, W. (2005) Reciprocal transcriptional regulation of Pou5f1 and Sox 2 via the Oct $4 /$ Sox 2 complex in embryonic stem cells. Molecular and Cellular Biology, 25 (14), 6031-6046.

(56) Chambers, I., Silva, J., Colby, D. et al. (2007) Nanog safeguards pluripotency and mediates germline development. Nature, 450 (7173), 1230-1234.

(57) Mitsui, K., Tokuzawa, Y., Itoh, H. et al. (2003) The homeoprotein Nanog is required for maintenance of pluripotency in mouse epiblast and ES cells. Cell, 113 (5), 631-642.

(58) Chen, L. and Daley, G.Q. (2008) Molecular basis of pluripotency. Human Molecular Genetics, 17 (R1), R23-27.

(59) Kim, J., Chu, J., Shen, X. et al. (2008) An extended transcriptional network for pluripotency of embryonic stem cells. Cell, 132 (6), 1049-1061. 
(60) Wang, Z., Oron, E., Nelson, B. et al. (2012) Distinct lineage specification roles for NANOG, OCT4, and SOX2 in human embryonic stem cells. Cell Stem Cell, 10 (4), 440-454.

(61) Lee, T.I., Jenner, R.G., Boyer, L.A. et al. (2006) Control of developmental regulators by Polycomb in human embryonic stem cells. Cell, 125 (2), 301-313.

(62) Endoh, M., Endo, T.A., Endoh, T. et al. (2008) Polycomb group proteins Ring1A/B are functionally linked to the core transcriptional regulatory circuitry to maintain ES cell identity. Development (Cambridge, England), 135 (8), 1513-1524.

(63) Kirmizis, A., Bartley, S.M., Kuzmichev, A. et al. (2004) Silencing of human polycomb target genes is associated with methylation of histone H3 Lys 27. Genes \& Development, 18 (13), 1592-1605.

(64) Bernstein, B.E., Mikkelsen, T.S., Xie, X. et al. (2006) A bivalent chromatin structure marks key developmental genes in embryonic stem cells. Cell, 125 (2), 315-326.

(65) Stock, J.K., Giadrossi, S., Casanova, M. et al. (2007) Ring1-mediated ubiquitination of H2A restrains poised RNA polymerase II at bivalent genes in mouse ES cells. Nature Cell Biology, 9 (12), 1428-1435.

(66) Dillon, N. (2012) Factor mediated gene priming in pluripotent stem cells sets the stage for lineage specification. BioEssays: News and Reviews in Molecular, Cellular and Developmental Biology, 34 (3), 194-204.

(67) Marson, A., Levine, S.S., Cole, M.F. et al. (2008) Connecting microRNA genes to the core transcriptional regulatory circuitry of embryonic stem cells. Cell, 134 (3), 521-533.

(68) Tay, Y.M., Tam, W.L., Ang, Y.S. et al. (2008) MicroRNA-134 modulates the differentiation of mouse embryonic stem cells, where it causes post-transcriptional attenuation of Nanog and LRH1. Stem Cells, 26 (1), 17-29.

(69) Khalil, A.M., Guttman, M., Huarte, M. et al. (2009) Many human large intergenic noncoding RNAs associate with chromatin-modifying complexes and affect gene expression. Proceedings of the National Academy of Sciences of the United States of America, 106 (28), 1166711672 .

(70) Guttman, M., Donaghey, J., Carey, B.W. et al. (2011) lincRNAs act in the circuitry controlling pluripotency and differentiation. Nature, 477 (7364), 295-300.

(71) Tollervey, J.R. and Lunyak, V.V. (2012) Epigenetics: judge, jury and executioner of stem cell fate. Epigenetics: Official Journal of the DNA Methylation Society, 7 (8), 823-840.

(72) Meissner, A. (2010) Epigenetic modifications in pluripotent and differentiated cells. Nature Biotechnology, 28 (10), 1079-1088.

(73) O'Carroll, D., Erhardt, S., Pagani, M. et al. (2001) The polycomb-group gene Ezh2 is required for early mouse development. Molecular and Cellular Biology, 21 (13), 4330-4336.

(74) Boyer, L.A., Plath, K., Zeitlinger, J. et al. (2006) Polycomb complexes repress developmental regulators in murine embryonic stem cells. Nature, 441 (7091), 349-353.

(75) Hochedlinger, K. and Plath, K. (2009) Epigenetic reprogramming and induced pluripotency. Development (Cambridge, England), 136 (4), 509-523.

(76) Guenther, M.G., Frampton, G.M., Soldner, F. et al. (2010) Chromatin structure and gene expression programs of human embryonic and induced pluripotent stem cells. Cell Stem Cell, 7 (2), 249-257.

(77) Papp, B. and Plath, K. (2011) Reprogramming to pluripotency: stepwise resetting of the epigenetic landscape. Cell Research, 21 (3), 486-501.

(78) Vierbuchen, T. and Wernig, M. (2012) Molecular roadblocks for cellular reprogramming. Molecular Cell, 47 (6), 827-838.

(79) Maherali, N., Sridharan, R., Xie, W. et al. (2007) Directly reprogrammed fibroblasts show global epigenetic remodeling and widespread tissue contribution. Cell Stem Cell, 1 (1), $55-70$.

(80) Wernig, M., Meissner, A., Foreman, R. et al. (2007) In vitro reprogramming of fibroblasts into a pluripotent ES-cell-like state. Nature, 448 (7151), 318-324.

(81) Chin, M.H., Mason, M.J., Xie, W. et al. (2009) Induced pluripotent stem cells and embryonic stem cells are distinguished by gene expression signatures. Cell Stem Cell, 5 (1), 111123.

(82) Lister, R., Pelizzola, M., Kida, Y.S. et al. (2011) Hotspots of aberrant epigenomic reprogramming in human induced pluripotent stem cells. Nature, 471 (7336), 68-73. 
(83) Wang, A., Huang, K., Shen, Y. et al. (2011) Functional modules distinguish human induced pluripotent stem cells from embryonic stem cells. Stem Cells and Development, 20 (11), 1937-1950.

(84) Marchetto, M.C., Yeo, G.W., Kainohana, O. et al. (2009) Transcriptional signature and memory retention of human-induced pluripotent stem cells. PloS One, 4 (9), e7076.

(85) Kim, K., Doi, A., Wen, B. et al. (2010) Epigenetic memory in induced pluripotent stem cells. Nature, 467 (7313), 285-290.

(86) Polo, J.M., Liu, S., Figueroa, M.E. et al. (2010) Cell type of origin influences the molecular and functional properties of mouse induced pluripotent stem cells. Nature Biotechnology, 28 (8), 848-855.

(87) Sullivan, G.J., Bai, Y., Fletcher, J., and Wilmut, I. (2010) Induced pluripotent stem cells: epigenetic memories and practical implications. Molecular Human Reproduction, 16 (12), $880-885$.

(88) Nakagawa, M., Koyanagi, M., Tanabe, K. et al. (2008) Generation of induced pluripotent stem cells without Myc from mouse and human fibroblasts. Nature Biotechnology, 26 (1), 101-106.

(89) Kim, J.B., Greber, B., Arauzo-Bravo, M.J. et al. (2009) Direct reprogramming of human neural stem cells by OCT4. Nature, 461 (7264), 649-643.

(90) Sterneckert, J., Höing, S., and Schöler, H.R. (2012) Concise review: Oct4 and more: the reprogramming expressway. Stem Cells, 30 (1), 15-21.

(91) Kuroda, T., Tada, M., Kubota, H. et al. (2005) Octamer and Sox elements are required for transcriptional cis regulation of Nanog gene expression. Molecular and Cellular Biology, 25 (6), 2475-2485.

(92) Nakatake, Y., Fukui, N., Iwamatsu, Y. et al. (2006) Klf4 cooperates with Oct3/4 and Sox 2 to activate the Lefty 1 core promoter in embryonic stem cells. Molecular and Cellular Biology, 26 (20), 7772-7782.

(93) Fong, Y.W., Inouye, C., Yamaguchi, T. et al. (2011) A DNA repair complex functions as an Oct4/Sox2 coactivator in embryonic stem cells. Cell, 147 (1), 120-131.

(94) Cao, Q., Zhang, X., Lu, L. et al. (2012) Klf4 is required for germ-layer differentiation and body axis patterning during Xenopus embryogenesis. Development (Cambridge, England), 139 (21), 3950-3961.

(95) Ghaleb, A.M., McConnell, B.B., Kaestner, K.H., and Yang, V.W. (2011) Altered intestinal epithelial homeostasis in mice with intestine-specific deletion of the Kruppel-like factor 4 gene. Developmental Biology, 349 (2), 310-320.

(96) Zhang, W., Chen, X., Kato, Y. et al. (2006) Novel cross talk of Kruppel-like factor 4 and betacatenin regulates normal intestinal homeostasis and tumor repression. Molecular and Cellular Biology, 26 (6), 2055-2064.

(97) Yang, W.T. and Zheng, P.S. (2012) Kruppel-like factor 4 functions as a tumor suppressor in cervical carcinoma. Cancer, 118 (15), 3691-3702.

(98) Kaushik, D.K., Gupta, M., Das, S., and Basu, A. (2010) Kruppel-like factor 4, a novel transcription factor regulates microglial activation and subsequent neuroinflammation. Journal of Neuroinflammation, 7, 68.

(99) Chang, C.W., Lai, Y.S., Pawlik, K.M. et al. (2009) Polycistronic lentiviral vector for "Hit and Run" reprogramming of adult skin fibroblasts to induced pluripotent stem cells. Stem Cells, 27 (5), 1042-1049.

(100) Papapetrou, E.P., Tomishima, M.J., Chambers, S.M. et al. (2009) Stoichiometric and temporal requirements of Oct4, Sox2, Klf4, and c-Myc expression for efficient human iPSC induction and differentiation. Proceedings of the National Academy of Sciences of the United States of America, 106 (31), 12759-12764.

(101) Wei, Z., Yang, Y., Zhang, P. et al. (2009) Klf4 interacts directly with Oct4 and Sox 2 to promote reprogramming. Stem Cells, 27 (12), 2969-2978.

(102) Morrish, F., Neretti, N., Sedivy, J.M., and Hockenbery, D.M. (2008) The oncogene c-Myc coordinates regulation of metabolic networks to enable rapid cell cycle entry. Cell Cycle, 7 (8), 1054-1066.

(103) Miller, D.M., Thomas, S.D., Islam, A. et al. (2012) c-Myc and cancer metabolism. Clinical cancer Research: An Official Journal of the American Association for Cancer Research, 18 (20), 5546-5553. 
(104) Cartwright, P., McLean, C., Sheppard, A. et al. (2005) LIF/STAT3 controls ES cell self-renewal and pluripotency by a Myc-dependent mechanism. Development (Cambridge, England), 132 (5), 885-896.

(105) Fernandez, P.C., Frank, S.R., Wang, L. et al. (2003) Genomic targets of the human c-Myc protein. Genes \& Development, 17 (9), 1115-1129.

(106) Lin, C.Y., Loven, J., Rahl, P.B. et al. (2012) Transcriptional amplification in tumor cells with elevated c-Myc. Cell, 151 (1), 56-67.

(107) Wernig, M., Meissner, A., Cassady, J.P., and Jaenisch, R. (2008) c-Myc is dispensable for direct reprogramming of mouse fibroblasts. Cell Stem Cell, 2 (1), 10-12.

(108) Liao, J., Wu, Z., Wang, Y. et al. (2008) Enhanced efficiency of generating induced pluripotent stem (iPS) cells from human somatic cells by a combination of six transcription factors. Cell Research, 18 (5), 600-603.

(109) Huangfu, D., Osafune, K., Maehr, R. et al. (2008) Induction of pluripotent stem cells from primary human fibroblasts with only Oct4 and Sox2. Nature Biotechnology, 26 (11), 12691275.

(110) Kim, J.B., Zaehres, H., Arauzo-Bravo, M.J., and Scholer, H.R. (2009) Generation of induced pluripotent stem cells from neural stem cells. Nature Protocols, 4 (10), 1464-1470.

(111) Heng, J.C.D., Feng, B., Han, J.Y. et al. (2010) The nuclear receptor Nr5a2 can replace Oct4 in the reprogramming of murine somatic cells to pluripotent cells. Cell Stem Cell, 6 (2), 167-174.

(112) Wang, W., Yang, J., Liu, H. et al. (2011) Rapid and efficient reprogramming of somatic cells to induced pluripotent stem cells by retinoic acid receptor gamma and liver receptor homolog 1. Proceedings of the National Academy of Sciences of the United States of America, 108 (45), 18283-18288.

(113) Xu, Y., Wei, X., Wang, M. et al. (2013) Proliferation rate of somatic cells affects reprogramming efficiency. The Journal of Biological Chemistry, 288 (14), 9767-9778.

(114) Carey, B.W., Markoulaki, S., Hanna, J.H. et al. (2011) Reprogramming factor stoichiometry influences the epigenetic state and biological properties of induced pluripotent stem cells. Cell Stem Cell, 9 (6), 588-598.

(115) Nagamatsu, G., Saito, S., Kosaka, T. et al. (2012) Optimal ratio of transcription factors for somatic cell reprogramming. The Journal of Biological Chemistry, 287 (43), 36273-36282.

(116) Tiemann, U., Sgodda, M., Warlich, E. et al. (2011) Optimal reprogramming factor stoichiometry increases colony numbers and affects molecular characteristics of murine induced pluripotent stem cells. Cytometry. Part A: The Journal of the International Society for Analytical Cytology, 79 (6), 426-435.

(117) Brambrink, T., Foreman, R., Welstead, G.G. et al. (2008) Sequential expression of pluripotency markers during direct reprogramming of mouse somatic cells. Cell Stem Cell, 2 (2), 151-159.

(118) Aasen, T., Raya, A., Barrero, M.J. et al. (2008) Efficient and rapid generation of induced pluripotent stem cells from human keratinocytes. Nature Biotechnology, 26 (11), 1276-1284.

(119) Giorgetti, A., Montserrat, N., Aasen, T. et al. (2009) Generation of induced pluripotent stem cells from human cord blood using OCT4 and SOX2. Cell Stem Cell, 5 (4), 353-357.

(120) Okita, K., Nakagawa, M., Hyenjong, H. et al. (2008) Generation of mouse induced pluripotent stem cells without viral vectors. Science, 322 (5903), 949-953.

(121) Fusaki, N., Ban, H., Nishiyama, A. et al. (2009) Efficient induction of transgene-free human pluripotent stem cells using a vector based on Sendai virus, an RNA virus that does not integrate into the host genome. Proceedings of the Japan Academy - Series B: Physical \& Biological Sciences, 85 (8), 348-362.

(122) Stadtfeld, M., Nagaya, M., Utikal, J. et al. (2008) Induced pluripotent stem cells generated without viral integration. Science, 322 (5903), 945-949.

(123) Warren, L., Manos, P.D., Ahfeldt, T. et al. (2010) Highly efficient reprogramming to pluripotency and directed differentiation of human cells with synthetic modified mRNA. Cell Stem Cell, 7 (5), 618-630.

(124) Kim, D., Kim, C.H., Moon, J.I. et al. (2009) Generation of human induced pluripotent stem cells by direct delivery of reprogramming proteins. Cell Stem Cell, 4 (6), 472-476.

(125) Haase, A., Olmer, R., Schwanke, K. et al. (2009) Generation of induced pluripotent stem cells from human cord blood. Cell Stem Cell, 5 (4), 434-441. 
(126) Sommer, C.A., Stadtfeld, M., Murphy, G.J. et al. (2009) Induced pluripotent stem cell generation using a single lentiviral stem cell cassette. Stem Cells, 27 (3), 543-549.

(127) Sommer, C.A., Sommer, A.G., Longmire, T.A. et al. (2010) Excision of reprogramming transgenes improves the differentiation potential of iPS cells generated with a single excisable vector. Stem Cells, 28 (1), 64-74.

(128) Gonzalez, F., Boue, S. and Izpisua Belmonte, J.C. (2011) Methods for making induced pluripotent stem cells: reprogramming a la carte. Nature Reviews Genetics, 12 (4), 231-242.

(129) Zhou, W.B. and Freed, C.R. (2009) Adenoviral gene delivery can reprogram human fibroblasts to induced pluripotent stem cells. Stem Cells, 27 (11), 2667-2674.

(130) Nishimura, K., Sano, M., Ohtaka, M. et al. (2011) Development of defective and persistent Sendai virus vector: a unique gene delivery/expression system ideal for cell reprogramming. The Journal of Biological Chemistry, 286 (6), 4760-4771.

(131) Soldner, F., Hockemeyer, D., Beard, C. et al. (2009) Parkinson's disease patient-derived induced pluripotent stem cells free of viral reprogramming factors. Cell, 136 (5), 964-977.

(132) Yu, J., Hu, K., Smuga-Otto, K. et al. (2009) Human induced pluripotent stem cells free of vector and transgene sequences. Science, 324 (5928), 797-801.

(133) Jia, F., Wilson, K.D., Sun, N. et al. (2010) A nonviral minicircle vector for deriving human iPS cells. Nature Methods, 7 (3), 197-199.

(134) Yusa, K., Rad, R., Takeda, J., and Bradley, A. (2009) Generation of transgene-free induced pluripotent mouse stem cells by the piggyBac transposon. Nature Methods, 6 (5), 363-369.

(135) Woltjen, K., Hamalainen, R., Kibschull, M. et al. (2011) Transgene-free production of pluripotent stem cells using piggyBac transposons. Methods in Molecular Biology, 767, 87-103.

(136) Yakubov, E., Rechavi, G., Rozenblatt, S., and Givol, D. (2010) Reprogramming of human fibroblasts to pluripotent stem cells using mRNA of four transcription factors. Biochemical and Biophysical Research Communications, 394 (1), 189-193.

(137) Zhou, H., Wu, S., Joo, J. et al. (2009) Generation of induced pluripotent stem cells using recombinant proteins. Cell Stem Cell, 4 (5), 381-384. Epub 2009 Apr 2023.

(138) Miyoshi, N., Ishii, H., Nagano, H. et al. (2011) Reprogramming of mouse and human cells to pluripotency using mature microRNAs. Cell Stem Cell, 8 (6), 633-638.

(139) Anokye-Danso, F., Trivedi, C.M., Juhr, D. et al. (2011) Highly efficient miRNA-mediated reprogramming of mouse and human somatic cells to pluripotency. Cell Stem Cell, 8 (4), 376-388.

(140) Sommer, C.A., Christodoulou, C., Gianotti-Sommer, A. et al. (2012) Residual expression of reprogramming factors affects the transcriptional program and epigenetic signatures of induced pluripotent stem cells. PLoS One, 7 (12), e51711.

(141) Ramos-Mejia, V., Montes, R., Bueno, C. et al. (2012) Residual expression of the reprogramming factors prevents differentiation of iPSC generated from human fibroblasts and cord blood CD34 + progenitors. PLoS One, 7 (4), e35824.

(142) Lohle, M., Hermann, A., Glass, H. et al. (2012) Differentiation efficiency of induced pluripotent stem cells depends on the number of reprogramming factors. Stem Cells, 30 (3), 570-579.

(143) Chou, B.K., Mali, P., Huang, X. et al. (2011) Efficient human iPS cell derivation by a nonintegrating plasmid from blood cells with unique epigenetic and gene expression signatures. Cell Research, 21 (3), 518-529.

(144) Yu, J., Chau, K.F., Vodyanik, M.A. et al. (2011) Efficient feeder-free episomal reprogramming with small molecules. PloS One, 6 (3), e17557.

(145) Gonzalez, F., Barragan Monasterio, M., Tiscornia, G. et al. (2009) Generation of mouseinduced pluripotent stem cells by transient expression of a single nonviral polycistronic vector. Proceedings of the National Academy of Sciences of the United States of America, 106 (22), 8918-8922.

(146) Warren, L., Ni, Y., Wang, J., and Guo, X. (2012) Feeder-free derivation of human induced pluripotent stem cells with messenger RNA. Scientific Reports, $2,657$.

(147) Fish, J.E., Santoro, M.M., Morton, S.U. et al. (2008) miR-126 regulates angiogenic signaling and vascular integrity. Developmental Cell, 15 (2), 272-284.

(148) Goossens, K., Mestdagh, P., Lefever, S. et al. (2013) Regulatory microRNA network identification in bovine blastocyst development. Stem Cells and Development, 22 (13), 1907-1920. 
(149) Li, Q. and Gregory, R.I. (2008) MicroRNA regulation of stem cell fate. Cell Stem Cell, 2 (3), 195-196.

(150) O'Connell, R.M. and Baltimore, D. (2012) MicroRNAs and hematopoietic cell development. Current Topics in Developmental Biology, 99, 145-174.

(151) Cordes, K.R. and Srivastava, D. (2009) MicroRNA regulation of cardiovascular development. Circulation Research, 104 (6), 724-732.

(152) Judson, R.L., Babiarz, J.E., Venere, M., and Blelloch, R. (2009) Embryonic stem cell-specific microRNAs promote induced pluripotency. Nature Biotechnology, 27 (5), 459-461.

(153) Zhang, Z. and Wu, W.S. (2013) Sodium butyrate promotes generation of human iPS cells through induction of the miR302/367 cluster. Stem Cells and Development, 22 (16), 22682277.

(154) Chen, J., Wang, G., Lu, C. et al. (2012) Synergetic cooperation of microRNAs with transcription factors in iPS cell generation. PLoS One, 7 (7), e 40849.

(155) Belting, M., Sandgren, S., and Wittrup, A. (2005) Nuclear delivery of macromolecules: barriers and carriers. Advanced Drug Delivery Reviews, 57 (4), 505-527.

(156) Frankel, A.D. and Pabo, C.O. (1988) Cellular uptake of the tat protein from human immunodeficiency virus. Cell, 55 (6), 1189-1193.

(157) El-Sayed, A., Masuda, T., Khalil, I. et al. (2009) Enhanced gene expression by a novel stearylated INF7 peptide derivative through fusion independent endosomal escape. Journal of Controlled Release: Official Journal of the Controlled Release Society, 138 (2), 160-167.

(158) Li, W., Wei, W., Zhu, S. et al. (2009) Generation of rat and human induced pluripotent stem cells by combining genetic reprogramming and chemical inhibitors. Cell Stem Cell, 4 (1), $16-19$.

(159) Zhao, Y., Yin, X., Qin, H. et al. (2008) Two supporting factors greatly improve the efficiency of human iPSC generation. Cell Stem Cell, 3 (5), 475-479.

(160) Lin, T., Ambasudhan, R., Yuan, X. et al. (2009) A chemical platform for improved induction of human iPSCs. Nature Methods, 6 (11), 805-808.

(161) Hanna, J., Saha, K., Pando, B., and van Zon, J. (2009) Direct cell reprogramming is a stochastic process amenable to acceleration. Nature, 462 (7273), 595-601.

(162) Xu, Y., Zhu, X., Hahm, H.S. et al. (2010) Revealing a core signaling regulatory mechanism for pluripotent stem cell survival and self-renewal by small molecules. Proceedings of the National Academy of Sciences of the United States of America, 107 (18), 8129-8134.

(163) Ichida, J.K., Blanchard, J., Lam, K. et al. (2009) A small-molecule inhibitor of tgf-Beta signaling replaces sox 2 in reprogramming by inducing nanog. Cell Stem Cell, 5 (5), 491-503.

(164) Mali, P., Chou, B.K., Yen, J. et al. (2010) Butyrate greatly enhances derivation of human induced pluripotent stem cells by promoting epigenetic remodeling and the expression of pluripotency-associated genes. Stem Cells, 28 (4), 713-720.

(165) Zhang, Z., Gao, Y., Gordon, A. et al. (2011) Efficient generation of fully reprogrammed human iPS cells via polycistronic retroviral vector and a new cocktail of chemical compounds. PloS One, 6 (10), e26592.

(166) Li, W., Zhou, H., Abujarour, R. et al. (2009) Generation of human-induced pluripotent stem cells in the absence of exogenous Sox2. Stem Cells, 27 (12), 2992-3000.

(167) Shi, Y., Do, J.T., Desponts, C. et al. (2008) A combined chemical and genetic approach for the generation of induced pluripotent stem cells. Cell Stem Cell, 2 (6), 525-528.

(168) Park, I.H., Zhao, R., West, J.A. et al. (2008) Reprogramming of human somatic cells to pluripotency with defined factors. Nature, 451 (7175), 141-146.

(169) Shi, Y., Desponts, C., Do, J.T. et al. (2008) Induction of pluripotent stem cells from mouse embryonic fibroblasts by Oct4 and Klf4 with small-molecule compounds. Cell Stem Cell, 3 (5), 568-574.

(170) Yoshida, M., Kijima, M., Akita, M., and Beppu, T. (1990) Potent and specific inhibition of mammalian histone deacetylase both in vivo and in vitro by trichostatin A. The Journal of Biological Chemistry, 265 (28), 17174-17179.

(171) Lyssiotis, C.A., Foreman, R.K., Staerk, J. et al. (2009) Reprogramming of murine fibroblasts to induced pluripotent stem cells with chemical complementation of Klf4. Proceedings of the National Academy of Sciences of the United States of America, 106 (22), 8912-8917. 
(172) Waddington, C.H. (1957) The Strategy of the Genes, Allen \& Unwin.

(173) Takahashi, K. (2012) Cellular reprogramming-lowering gravity on Waddington's epigenetic landscape. Journal of Cell Science, 125 (Pt 11), 2553-2560.

(174) Davis, R.L., Weintraub, H., and Lassar, A.B. (1987) Expression of a single transfected cDNA converts fibroblasts to myoblasts. Cell, $\mathbf{5 1}$ (6), 987-1000.

(175) Zhou, Q., Brown, J., Kanarek, A. et al. (2008) In vivo reprogramming of adult pancreatic exocrine cells to beta-cells. Nature, 455 (7213), 627-632.

(176) Vierbuchen, T., Ostermeier, A., Pang, Z.P. et al. (2010) Direct conversion of fibroblasts to functional neurons by defined factors. Nature, $\mathbf{4 6 3}$ (7284), 1035-1041.

(177) Huang, P., He, Z., Ji, S. et al. (2011) Induction of functional hepatocyte-like cells from mouse fibroblasts by defined factors. Nature, 475 (7356), 386-389.

(178) Ieda, M., Fu, J.D., Delgado-Olguin, P. et al. (2010) Direct reprogramming of fibroblasts into functional cardiomyocytes by defined factors. Cell, 142 (3), 375-386.

(179) Szabo, E., Rampalli, S., Risueno, R.M. et al. (2010) Direct conversion of human fibroblasts to multilineage blood progenitors. Nature, 468 (7323), 521-526.

(180) Adler, A.F., Grigsby, C.L., Kulangara, K. et al. (2012) Nonviral direct conversion of primary mouse embryonic fibroblasts to neuronal cells. Molecular Therapy. Nucleic Acids, $\mathbf{1}$, e32.

(181) Bichsel, C., Neeld, D., Hamazaki, T. et al. (2013) Direct reprogramming of fibroblasts to myocytes via bacterial injection of MyoD protein. Cellular Reprogramming, 15 (2), 117 125.

(182) Xue, Y., Ouyang, K., Huang, J. et al. (2013) Direct conversion of fibroblasts to neurons by reprogramming PTB-regulated microRNA circuits. Cell, 152 (1-2), 82-96.

(183) Zhu, Z. and Huangfu, D. (2013) Human pluripotent stem cells: an emerging model in developmental biology. Development (Cambridge, England), 140 (4), 705-717.

(184) Nostro, M.C., Sarangi, F., Ogawa, S. et al. (2011) Stage-specific signaling through TGFbeta family members and WNT regulates patterning and pancreatic specification of human pluripotent stem cells. Development (Cambridge, England), 138 (5), 861-871.

(185) Hu, B.Y., Weick, J.P., Yu, J. et al. (2010) Neural differentiation of human induced pluripotent stem cells follows developmental principles but with variable potency. Proceedings of the National Academy of Sciences of the United States of America, 107 (9), 4335-4340.

(186) Gore, A., Li, Z., Fung, H.L. et al. (2011) Somatic coding mutations in human induced pluripotent stem cells. Nature, 471 (7336), 63-67.

(187) Hussein, S.M., Batada, N.N., Vuoristo, S. et al. (2011) Copy number variation and selection during reprogramming to pluripotency. Nature, 471 (7336), 58-62.

(188) Laurent, L.C., Ulitsky, I., Slavin, I. et al. (2011) Dynamic changes in the copy number of pluripotency and cell proliferation genes in human ESCs and iPSCs during reprogramming and time in culture. Cell Stem Cell, 8 (1), 106-118.

(189) Nazor, K.L., Altun, G., Lynch, C. et al. (2012) Recurrent variations in DNA methylation in human pluripotent stem cells and their differentiated derivatives. Cell Stem Cell, 10 (5), 620-634.

(190) Grskovic, M., Javaherian, A., Strulovici, B., and Daley, G.Q. (2011) Induced pluripotent stem cells-opportunities for disease modelling and drug discovery. Nature Reviews Drug Discovery, 10 (12), 915-929.

(191) Robinton, D.A. and Daley, G.Q. (2012) The promise of induced pluripotent stem cells in research and therapy. Nature, 481 (7381), 295-305.

(192) Bellin, M., Marchetto, M.C., Gage, F.H., and Mummery, C.L. (2012) Induced pluripotent stem cells: the new patient? Nature Reviews Molecular Cell Biology, 13 (11), 713-726.

(193) Brock, A., Goh, H.T., Yang, B. et al. (2012) Cellular reprogramming: a new technology frontier in pharmaceutical research. Pharmaceutical Research, 29 (1), 35-52.

(194) Moran, N. (2013) Banking iPS cells. Nature Biotechnology, 31 (1), 11-11.

(195) Somers, A., Jean, J.C., Sommer, C.A. et al. (2010) Generation of transgene-free lung diseasespecific human induced pluripotent stem cells using a single excisable lentiviral stem cell cassette. Stem Cells, 28 (10), 1728-1740.

(196) Yagi, T., Ito, D., Okada, Y. et al. (2011) Modeling familial Alzheimer's disease with induced pluripotent stem cells. Human Molecular Genetics, 20 (23), 4530-4539. 
(197) Israel, M.A., Yuan, S.H., Bardy, C. et al. (2012) Probing sporadic and familial Alzheimer's disease using induced pluripotent stem cells. Nature, 482 (7384), 216-220.

(198) Dimos, J.T., Rodolfa, K.T., Niakan, K.K. et al. (2008) Induced pluripotent stem cells generated from patients with ALS can be differentiated into motor neurons. Science, 321 (5893), 12181221.

(199) Bilican, B., Serio, A., Barmada, S.J. et al. (2012) Mutant induced pluripotent stem cell lines recapitulate aspects of TDP-43 proteinopathies and reveal cell-specific vulnerability. Proceedings of the National Academy of Sciences of the United States of America, 109 (15), 5803-5808.

(200) Mitne-Neto, M., Machado-Costa, M., Marchetto, M.C. et al. (2011) Downregulation of VAPB expression in motor neurons derived from induced pluripotent stem cells of ALS8 patients. Human Molecular Genetics, 20 (18), 3642-3652.

(201) Chamberlain, S.J., Chen, P.F., Ng, K.Y. et al. (2010) Induced pluripotent stem cell models of the genomic imprinting disorders Angelman and Prader-Willi syndromes. Proceedings of the National Academy of Sciences of the United States of America, 107 (41), 17668-17673.

(202) Ma, D., Wei, H., Lu, J. et al. (2013) Generation of patient-specific induced pluripotent stem cell-derived cardiomyocytes as a cellular model of arrhythmogenic right ventricular cardiomyopathy. European Heart Journal, 34 (15), 1122-1133.

(203) Nayler, S., Gatei, M., Kozlov, S. et al. (2012) Induced pluripotent stem cells from ataxiatelangiectasia recapitulate the cellular phenotype. Stem Cells Translational Medicine, 1 (7), $523-535$.

(204) Ho, J.C., Zhou, T., Lai, W.H. et al. (2011) Generation of induced pluripotent stem cell lines from 3 distinct laminopathies bearing heterogeneous mutations in lamin A/C. Aging, 3 (4), 380-390.

(205) Park, I.H., Arora, N., Huo, H. et al. (2008) Disease-specific induced pluripotent stem cells. Cell, 134 (5), 877-886.

(206) Singh, R., Shen, W., Kuai, D. et al. (2013) iPS cell modeling of Best disease: insights into the pathophysiology of an inherited macular degeneration. Human Molecular Genetics, 22 (3), 593-607.

(207) Wang, Y., Jiang, Y., Liu, S., Sun, X., and Gao, S. (2009) Generation of induced pluripotent stem cells from human beta-thalassemia fibroblast cells. Cell Research, 19 (9), 1120-1123.

(208) Pessach, I.M., Ordovas-Montanes, J., Zhang, S.Y. et al. (2011) Induced pluripotent stem cells: a novel frontier in the study of human primary immunodeficiencies. The Journal of Allergy and Clinical Immunology, 127 (6), 1400-1407, e1404.

(209) Fatima, A., Xu, G., Shao, K. et al. (2011) In vitro modeling of ryanodine receptor 2 dysfunction using human induced pluripotent stem cells. Cellular Physiology and Biochemistry, 28 (4), 579-592.

(210) Novak, A., Barad, L., Zeevi-Levin, N. et al. (2012) Cardiomyocytes generated from CPVTD307H patients are arrhythmogenic in response to beta-adrenergic stimulation. Journal of Cellular and Molecular Medicine, 16 (3), 468-482.

(211) Tanaka, T., Takahashi, K., Yamane, M. et al. (2012) Induced pluripotent stem cells from CINCA syndrome patients as a model for dissecting somatic mosaicism and drug discovery. Blood, 120 (6), 1299-1308.

(212) Andrade, L.N., Nathanson, J.L., Yeo, G.W. et al. (2012) Evidence for premature aging due to oxidative stress in iPSCs from Cockayne syndrome. Human Molecular Genetics, 21 (17), 3825-3834.

(213) Ghodsizadeh, A., Taei, A., Totonchi, M. et al. (2010) Generation of liver disease-specific induced pluripotent stem cells along with efficient differentiation to functional hepatocyte-like cells. Stem Cell Reviews, 6 (4), 622-632.

(214) Maehr, R., Chen, S., Snitow, M. et al. (2009) Generation of pluripotent stem cells from patients with type 1 diabetes. Proceedings of the National Academy of Sciences of the United States of America, 106 (37), 15768-15773.

(215) Kudva, Y.C., Ohmine, S., Greder, L.V. et al. (2012) Transgene-free disease-specific induced pluripotent stem cells from patients with type 1 and type 2 diabetes. Stem Cells Translational Medicine, 1 (6), 451-461. 
(216) Sun, N., Yazawa, M., Liu, J. et al. (2012) Patient-specific induced pluripotent stem cells as a model for familial dilated cardiomyopathy. Science Translational Medicine, 4 (130), 130ra147.

(217) Mou, X., Wu, Y., Cao, H. et al. (2012) Generation of disease-specific induced pluripotent stem cells from patients with different karyotypes of Down syndrome. Stem Cell Research \& Therapy, 3 (2), 14.

(218) Agarwal, S., Loh, Y.H., McLoughlin, E.M. et al. (2010) Telomere elongation in induced pluripotent stem cells from dyskeratosis congenita patients. Nature, 464 (7286), 292-296.

(219) Lee, G., Papapetrou, E.P., Kim, H. et al. (2009) Modelling pathogenesis and treatment of familial dysautonomia using patient-specific iPSCs. Nature, 461 (7262), 402-U100.

(220) Rashid, S.T., Corbineau, S., Hannan, N. et al. (2010) Modeling inherited metabolic disorders of the liver using human induced pluripotent stem cells. Journal of Clinical Investigation, 120 (9), 3127-3136.

(221) Lan, F., Lee, A.S., and Liang, P. (2013) Abnormal calcium handling properties underlie familial hypertrophic cardiomyopathy pathology in patient-specific induced pluripotent stem cells. Cell Stem Cell, 12 (1), 101-113.

(222) Raya, A., Rodriguez-Piza, I., Guenechea, G. et al. (2009) Disease-corrected haematopoietic progenitors from Fanconi anaemia induced pluripotent stem cells. Nature, 460 (7251), 53-59.

(223) Snider, L., Geng, L.N., Lemmers, R.J. et al. (2010) Facioscapulohumeral dystrophy: incomplete suppression of a retrotransposed gene. PLoS Genetics, 6 (10), e1001181.

(224) Urbach, A., Bar-Nur, O., Daley, G.Q., and Benvenisty, N. (2010) Differential modeling of fragile $\mathrm{X}$ syndrome by human embryonic stem cells and induced pluripotent stem cells. Cell Stem Cell, 6 (5), 407-411.

(225) Liu, J., Verma, P.J., Evans-Galea, M.V. et al. (2011) Generation of induced pluripotent stem cell lines from Friedreich ataxia patients. Stem Cell Reviews, 7 (3), 703-713.

(226) Panicker, L.M., Miller, D., Park, T.S. et al. (2012) Induced pluripotent stem cell model recapitulates pathologic hallmarks of Gaucher disease. Proceedings of the National Academy of Sciences of the United States of America, 109 (44), 18054-18059.

(227) Camnasio, S., Delli Carri, A., Lombardo, A. et al. (2012) The first reported generation of several induced pluripotent stem cell lines from homozygous and heterozygous Huntington's disease patients demonstrates mutation related enhanced lysosomal activity. Neurobiology of Disease, 46 (1), 41-51.

(228) Zhang, J., Lian, Q., Zhu, G. et al. (2011) A human iPSC model of Hutchinson Gilford Progeria reveals vascular smooth muscle and mesenchymal stem cell defects. Cell Stem Cell, 8 (1), $31-45$.

(229) Carvajal-Vergara, X., Sevilla, A., D'Souza, S.L. et al. (2010) Patient-specific induced pluripotent stem-cell-derived models of LEOPARD syndrome. Nature, 465 (7299), 808-812.

(230) Mekhoubad, S., Bock, C., de Boer, A.S. et al. (2012) Erosion of dosage compensation impacts human iPSC disease modeling. Cell Stem Cell, 10 (5), 595-609.

(231) Tedesco, F.S., Gerli, M.F., Perani, L. et al. (2012) Transplantation of genetically corrected human iPSC-derived progenitors in mice with limb-girdle muscular dystrophy. Science Translational Medicine, 4 (140), 140ra189.

(232) Moretti, A., Bellin, M., Welling, A. et al. (2010) Patient-specific induced pluripotent stem-cell models for long-QT syndrome. The New England Journal of Medicine, 363 (15), 1397-1409.

(233) Matsa, E., Rajamohan, D., Dick, E. et al. (2011) Drug evaluation in cardiomyocytes derived from human induced pluripotent stem cells carrying a long QT syndrome type 2 mutation. European Heart Journal, 32 (8), 952-962.

(234) Davis, R.P., Casini, S., vanden Berg, C.W. et al. (2012) Cardiomyocytes derived from pluripotent stem cells recapitulate electrophysiological characteristics of an overlap syndrome of cardiac sodium channel disease. Circulation, 125 (25), 3079-3091.

(235) Koch, P., Breuer, P., Peitz, M. et al. (2011) Excitation-induced ataxin-3 aggregation in neurons from patients with Machado-Joseph disease. Nature, 480 (7378), 543-546.

(236) Quarto, N., Leonard, B., Li, S. et al. (2012) Skeletogenic phenotype of human Marfan embryonic stem cells faithfully phenocopied by patient-specific induced-pluripotent stem cells. Proceedings of the National Academy of Sciences of the United States of America, 109 (1), 215-220. 
(237) Folmes, C.D., Martinez-Fernandez, A., Perales-Clemente, E. et al. (2013) Disease-causing Mitochondrial Heteroplasmy Segregated within Induced Pluripotent Stem Cell Clones Derived from A MELAS Patient. Stem Cells, 31 (7), 1298-1308.

(238) Tolar, J., Park, I.H., Xia, L. et al. (2011) Hematopoietic differentiation of induced pluripotent stem cells from patients with mucopolysaccharidosis type I (Hurler syndrome). Blood, 117 (3), 839-847.

(239) Lemonnier, T., Blanchard, S., Toli, D. et al. (2011) Modeling neuronal defects associated with a lysosomal disorder using patient-derived induced pluripotent stem cells. Human Molecular Genetics, 20 (18), 3653-3666.

(240) Ye, Z., Zhan, H., Mali, P. et al. (2009) Human-induced pluripotent stem cells from blood cells of healthy donors and patients with acquired blood disorders. Blood, 114 (27), 5473-5480.

(241) Deyle, D.R., Khan, I.F., Ren, G. et al. (2012) Normal collagen and bone production by genetargeted human osteogenesis imperfecta iPSCs. Molecular Therapy, 20 (1), 204-213.

(242) Hatano, T. (2013) Induced pluripotent stem cells can be a useful disease model for understanding the pathomechanisms of PARK2. Movement Disorders: Official Journal of the Movement Disorder Society, 28 (3), 289.

(243) Cooper, O., Seo, H., Andrabi, S. et al. (2012) Pharmacological rescue of mitochondrial deficits in iPSC-derived neural cells from patients with familial Parkinson's disease. Science Translational Medicine, 4 (141), 141ra190.

(244) Shimojima, K., Inoue, T., Imai, Y. et al. (2012) Reduced PLP1 expression in induced pluripotent stem cells derived from a Pelizaeus-Merzbacher disease patient with a partial PLP1 duplication. Journal of Human Genetics, 57 (9), 580-586.

(245) Huang, H.P., Chen, P.H., Hwu, W.L. et al. (2011) Human Pompe disease-induced pluripotent stem cells for pathogenesis modeling, drug testing and disease marker identification. Human Molecular Genetics, 20 (24), 4851-4864.

(246) Tolar, J., Xia, L., Riddle, M.J. et al. (2011) Induced pluripotent stem cells from individuals with recessive dystrophic epidermolysis bullosa. Journal of Investigative Dermatology, 131 (4), 848-856.

(247) Jin, Z.B., Okamoto, S., Osakada, F. et al. (2011) Modeling retinal degeneration using patientspecific induced pluripotent stem cells. PLoS One, 6 (2), e17084.

(248) Marchetto, M.C., Carromeu, C., Acab, A. et al. (2010) A model for neural development and treatment of Rett syndrome using human induced pluripotent stem cells. Cell, 143 (4), $527-539$.

(249) Ricciardi, S., Ungaro, F., Hambrock, M. et al. (2012) CDKL5 ensures excitatory synapse stability by reinforcing NGL-1-PSD95 interaction in the postsynaptic compartment and is impaired in patient iPSC-derived neurons. Nature Cell Biology, 14 (9), 911-923.

(250) Ogawa, Y., Tanaka, M., Tanabe, M. et al. (2013) Impaired neural differentiation of induced pluripotent stem cells generated from a mouse model of Sandhoff disease. PLoS One, 8 (1), e55856.

(251) Hiramoto, T., Ebihara, Y., Mizoguchi, Y. et al. (2013) Wnt3a stimulates maturation of impaired neutrophils developed from severe congenital neutropenia patient-derived pluripotent stem cells. Proceedings of the National Academy of Sciences of the United States of America, 110 (8), 3023-3028.

(252) Brennand, K.J., Simone, A., Jou, J. et al. (2011) Modelling schizophrenia using human induced pluripotent stem cells. Nature, 473 (7346), 221-225.

(253) Ebert, A.D., Yu, J., Rose, F.F. Jr. et al. (2009) Induced pluripotent stem cells from a spinal muscular atrophy patient. Nature, 457 (7227), 277-280.

(254) Xia, G., Santostefano, K., Hamazaki, T. et al. (2012) Generation of human-induced pluripotent stem cells to model spinocerebellar ataxia type 2 in vitro. Journal of Molecular Neuroscience, $51(2), 1-12$.

(255) Ge, X., Ren, Y., Bartulos, O. et al. (2012) Modeling supravalvular aortic stenosis syndrome with human induced pluripotent stem cells. Circulation, 126 (14), 1695-1704.

(256) Chen, Y., Luo, R., Xu, Y. et al. (2013) Generation of systemic lupus erythematosus-specific induced pluripotent stem cells from urine. Rheumatology International, Feb., 1-8. 
(257) Yazawa, M., Hsueh, B., Jia, X. et al. (2011) Using induced pluripotent stem cells to investigate cardiac phenotypes in Timothy syndrome. Nature, 471 (7337), 230-234.

(258) Kinnear, C., Chang, W.Y., Khattak, S. et al. (2013) Modeling and rescue of the vascular phenotype of Williams-Beuren syndrome in patient induced pluripotent stem cells. Stem Cells Translational Medicine, 2 (1), 2-15.

(259) Zhang, S., Chen, S., Li, W. et al. (2011) Rescue of ATP7B function in hepatocyte-like cells from Wilson's disease induced pluripotent stem cells using gene therapy or the chaperone drug curcumin. Human Molecular Genetics, 20 (16), 3176-3187.

(260) Jang, J., Kang, H.C., Kim, H.S. et al. (2011) Induced pluripotent stem cell models from X-linked adrenoleukodystrophy patients. Annals of Neurology, 70 (3), 402-409.

(261) Zou, J., Sweeney, C.L., Chou, B.K. et al. (2011) Oxidase-deficient neutrophils from $\mathrm{X}$-linked chronic granulomatous disease iPS cells: functional correction by zinc finger nuclease-mediated safe harbor targeting. Blood, 117 (21), 5561-5572.

(262) Yamanaka, S. (2007) Strategies and new developments in the generation of patient-specific pluripotent stem cells. Cell Stem Cell, 1 (1), 39-49.

(263) Hanna, J., Wernig, M., Markoulaki, S. et al. (2007) Treatment of sickle cell anemia mouse model with iPS cells generated from autologous skin. Science, 318 (5858), 1920-1923.

(264) Ben-David, U. and Benvenisty, N. (2011) The tumorigenicity of human embryonic and induced pluripotent stem cells. Nature Reviews Cancer, 11 (4), 268-277.

(265) Schwartz, S.D., Hubschman, J.P., Heilwell, G. et al. (2012) Embryonic stem cell trials for macular degeneration: a preliminary report. Lancet, 379 (9817), 713-720.

(266) Melville, H., Carpiniello, M., Hollis, K. et al. (2013) Stem cells: a new paradigm for disease modeling and developing therapies for age-related macular degeneration. Journal of Translational Medicine, 11 (1), 53.

(267) Cyranoski, D. (2013) Stem cells cruise to clinic. Nature, 494 (7438), 413.

(268) Thomas, K.R., Folger, K.R., and Capecchi, M.R. (1986) High frequency targeting of genes to specific sites in the mammalian genome. Cell, 44 (3), 419-428.

(269) Collin, J. and Lako, M. (2011) Concise review: putting a finger on stem cell biology: zinc finger nuclease-driven targeted genetic editing in human pluripotent stem cells. Stem Cells, 29 (7), 1021-1033.

(270) Hockemeyer, D., Wang, H.Y., Kiani, S. et al. (2011) Genetic engineering of human pluripotent cells using TALE nucleases. Nature Biotechnology, 29 (8), 731-734.

(271) Mali, P., Yang, L., Esvelt, K.M. et al. (2013) RNA-Guided Human Genome Engineering via Cas9. Science, 339 (6121), 823-826.

(272) Osborn, M.J., Starker, C.G., McElroy, A.N. et al. (2013) TALEN-based Gene Correction for Epidermolysis Bullosa. Molecular Therapy, 21 (6), 1151-1159.

(273) Ding, Q., Lee, Y.K., Schaefer, E.A. et al. (2013) A TALEN genome-editing system for generating human stem cell-based disease models. Cell Stem Cell, 12 (2), 238-251.

(274) Woltjen, K. and Stanford, W.L. (2009) Preview. Inhibition of Tgf-beta signaling improves mouse fibroblast reprogramming. Cell Stem Cell, 5 (5), 457-458.

(275) Yamanaka, S. (2009) Elite and stochastic models for induced pluripotent stem cell generation. Nature, 460 (7251), 49-52.

(276) Loring, J.F. and Rao, M.S. (2006) Establishing standards for the characterization of human embryonic stem cell lines. Stem Cells, 24 (1), 145-150.

(277) I. International Stem Cell, Adewumi, O., Aflatoonian, B., Ahrlund-Richter, L. et al. (2007) Characterization of human embryonic stem cell lines by the International Stem Cell Initiative. Nature Biotechnology, 25 (7), 803-816.

(278) Maherali, N., Ahfeldt, T., Rigamonti, A. et al. (2008) A high-efficiency system for the generation and study of human induced pluripotent stem cells. Cell Stem Cell, 3 (3), 340-345.

(279) Daley, G.Q., Lensch, M.W., Jaenisch, R. et al. (2009) Broader implications of defining standards for the pluripotency of iPSCs. Cell Stem Cell, 4 (3), 200-201; author reply 202.

(280) Reynolds, B.A. and Weiss, S. (1992) Generation of neurons and astrocytes from isolated cells of the adult mammalian central nervous system. Science, $\mathbf{2 5 5}$ (5052), 1707-1710. 


\title{
9 \\ Chemicals Facilitating Reprogramming
}

\author{
Zhong-Dong Shi, Federico González, and Danwei Huangfu \\ Developmental Biology Program, Memorial Sloan-Kettering Cancer Center, USA
}

\subsection{Introduction}

Human embryonic stem cells (hESCs) are derived from the inner cell mass (ICM) of cultured pre-implantation human blastocysts [1]. With their ability to proliferate indefinitely in vitro (self-renew), while maintaining the potential to differentiate into virtually any cell type of the human body (pluripotency), they provide an unlimited source of cells suitable for regenerative medicine, drug discovery, and the study of human development or disease [2]. The major limitation of hESCs for cell therapy resides in their origin. In addition to ethical controversies, transplantation of hESC-derived cells is complicated by potential immune responses to allogeneic tissues [3]. To ensure the histocompatibility needed for transplantation, it would be ideal to use pluripotent stem cells derived from the patient.

To accomplish this goal, somatic-cell nuclear transfer (SCNT) represents an attractive option. More than 3 decades separate the first tadpoles cloned by John Gurdon [4] from Dolly the Sheep, the first cloned mammal [5]. Since then, SCNT has allowed the generation of viable adult clones in many mammalian species [6-13] and permitted the creation of pluripotent stem cells from mouse [14], bovine [15], and rhesus macaque [16]. These successes suggest that the same approach could apply to humans. However, SCNT has yet to succeed in our species, due to the limited availability of human oocytes for research and potentially reflecting inherent barriers of the human model [17]. These problems have prevented the success of human therapeutic cloning up to now.

Chemical Biology in Regenerative Medicine: Bridging Stem Cells and Future Therapies, First Edition. Edited by Charles C. Hong, Ada S. Ao, and Jijun Hao.

(C) 2014 John Wiley \& Sons, Ltd. Published 2014 by John Wiley \& Sons, Ltd. 
SCNT [4] and somatic-cell fusion experiments with germ cells [18] or embryonic stem cells (ESCs) $[19,20]$ demonstrate that the somatic genome is amenable to reprogramming, and suggest that pluripotency can be restored in a terminally differentiated cell by factors present in oocytes or pluripotent stem cells. This concept led to the groundbreaking experiment by Yamanaka and colleagues, who managed to induce pluripotency in differentiated mouse and human adult fibroblasts by ectopically expressing four pluripotency-associated transcription factors: Oct4, Sox2, Klf4, and c-Myc [21,22]. This process, referred to as reprogramming, generates induced pluripotent stem cells (iPSC) that closely resemble ESCs in global gene-expression profile, epigenetic signature, and developmental potential [23-29]. Reprogramming represents a simple way of generating patient-specific human pluripotent stem cells (hPSCs) useful for a wide range of applications, including autologous cell-transplantation therapy, modeling of both monogenic and polygenic diseases, and provision of relevant cell types for drug toxicity, differentiation, and therapeutic screens.

These applications require efficient and reliable reprogramming methods, which are being optimized [30]. In most cases, the generation of human induced pluripotent stem cells (hiPSC) involves ectopic expression of a set of core pluripotency-related transcription factors (Oct4, Sox2, Klf4, and c-Myc, or other combinations) in adult fibroblasts over a course of $\sim 2-4$ weeks [22,27]. Reprogramming of human fibroblasts is a slow and inefficient process: less than $0.01 \%$ of donor cells become iPSCs in initial studies [31]. Hence, recent years have seen cohorts of publications aiming to improve the original protocol by using alternative donor cells [32-34] or identifying novel reprogramming factors [35-37]. So far, the most efficient protocols make use of constitutive- [22,27], or inducible- [38] integrative viral vectors to deliver the reprogramming factors. However, these approaches generate genetically and phenotypically heterogeneous cell lines, limiting their use for therapy due to concerns over tumorigenicity caused by potential insertional mutagenesis or reexpression of the reprogramming factors [25,39].

Various approaches have been developed to avoid potentially detrimental genetic modifications, using alternative methods to deliver reprogramming factors, such as integrationdefective viruses [40-42], episomes [43-46], transposons [47,48], cell-permeable recombinant proteins [49,50], RNAs [51], and microRNAs [52,53], often combined with treatment with chemical compounds that induce or facilitate reprogramming $[54,55]$. Beside this increasing number of alternative approaches, a mechanistic dissection of the process may reveal cell-intrinsic and -extrinsic signals governing reprogramming and enable the design of rational approaches to improve it.

In this regard, small molecules provide a powerful tool by which to address these issues. First, their biological effects are usually rapid, reversible, and dose-dependent, allowing a precise control of specific outcomes through modulation of their concentrations and combinations. Second, the structural diversity provided by synthetic chemistry facilitates functional optimization of compounds of interest. Third, compared to any genetic delivery method, the delivery of chemicals is both convenient and efficient. Using both phenotypic screening and hypothesis-driven approaches, many compounds have been identified that can functionally replace reprogramming transcription factors and enhance the efficiency and/or the kinetics of reprogramming by modulating the activity of epigenetic modifiers or signaling pathways controlling pluripotency or cell proliferation [56]. This chapter reviews recent findings that use chemical compounds to improve reprogramming. These chemical compounds and their roles in reprogramming are also summarized in Table 9.1. 
Table 9.1 Chemical compounds facilitating somatic cell reprogramming to pluripotency

\begin{tabular}{|c|c|c|}
\hline Category & $\begin{array}{l}\text { Chemical } \\
\text { compounds }\end{array}$ & Working conditions and effects \\
\hline \multirow[t]{8}{*}{$\begin{array}{l}\text { HDAC } \\
\text { inhibitor }\end{array}$} & VPA, TSA, SAHA & $\begin{array}{l}\text { Enhance iPSC formation from four-factor-infected MEFs } \\
\text { [54] }\end{array}$ \\
\hline & \multirow[t]{5}{*}{ VPA } & $\begin{array}{l}\text { Improves OSK-induced reprogramming and induces } \\
\text { iPSC generation from OS-infected human fibroblasts } \\
{[54,64]}\end{array}$ \\
\hline & & $\begin{array}{l}\text { Dramatically increases reprogramming efficiency of } \\
\text { MEFs transduced with OSKM under hypoxic } \\
\text { condition [63] }\end{array}$ \\
\hline & & $\begin{array}{l}\text { Promotes complete reprogramming and facilitates } \\
\text { expansion of fully reprogrammed iPSCs by inhibiting } \\
\text { growth of nonreprogrammed cells; generated iPSCs } \\
\text { can produce adult mice }[48,66]\end{array}$ \\
\hline & & $\begin{array}{l}\text { Enables reprogramming of MEFs to iPSCs using } \\
\text { recombinant proteins of four or three Yamanaka } \\
\text { factors (OSKM or OSK) [50] }\end{array}$ \\
\hline & & $\begin{array}{l}\text { Enables generation of iPSCs from MEFs by only } \\
\text { expressing miR302/367 [52] }\end{array}$ \\
\hline & \multirow[t]{2}{*}{$\mathrm{NaB}$} & $\begin{array}{l}\text { Enhances efficiency of iPSC generation from human } \\
\text { adult and fetal fibroblasts by retroviral delivery of four } \\
\text { or three Yamanaka factors (OSKM, OSK, OSM) [65] }\end{array}$ \\
\hline & & $\begin{array}{l}\text { Significantly improve reprogramming from MSCs } \\
\text { established from a patient with sickle-cell disease } \\
\text { using a piggyback transposon vector that contains } \\
\text { five transgenes (OSKM + Lin28) [65] }\end{array}$ \\
\hline \multirow[t]{3}{*}{$\begin{array}{l}\text { G9a HMTase } \\
\text { inhibitor }\end{array}$} & \multirow[t]{3}{*}{ BIX01294 } & $\begin{array}{l}\text { Enhances reprogramming efficiency of mouse NSCs } \\
\text { infected with OK [55] }\end{array}$ \\
\hline & & $\begin{array}{l}\text { Increases reprogramming using OK in MEFs when } \\
\text { combined with a DNMT inhibitor, RG108, and } \\
\text { L-calcium channel agonist, BayK8644 [69] }\end{array}$ \\
\hline & & $\begin{array}{l}\text { Enables reprogramming of mouse NSCs with SKM with } \\
\text { a very low efficiency [55] }\end{array}$ \\
\hline \multirow[t]{2}{*}{ LSD1 inhibitor } & \multirow[t]{2}{*}{ Parnate } & $\begin{array}{l}\text { Enables reprogramming of human keratinocytes } \\
\text { infected by KO when combined with CHIR99021 } \\
\text { [74] }\end{array}$ \\
\hline & & $\begin{array}{l}\text { Improves reprogramming of MEFs infected by Oct4 in } \\
\text { the presence of VPA, CHIR99021, and E-616452 [75] }\end{array}$ \\
\hline \multirow[t]{4}{*}{$\begin{array}{l}\text { DNMT } \\
\text { inhibitor }\end{array}$} & \multirow[t]{2}{*}{5 -aza } & $\begin{array}{l}\text { Increases the reprogramming efficiency of OSKM- or } \\
\text { OSK-infected MEFs [54] }\end{array}$ \\
\hline & & $\begin{array}{l}\text { Promotes partially reprogrammed MEFs to fully } \\
\text { reprogrammed iPSCs [24] }\end{array}$ \\
\hline & \multirow[t]{2}{*}{ RG108 } & $\begin{array}{l}\text { Enhances reprogramming efficiency of MEFs transduced } \\
\text { with OK when combined with BIX [69] }\end{array}$ \\
\hline & & $\begin{array}{l}\text { Increases reprogramming efficiency of human } \\
\text { fibroblasts transduced with OSKM [65] }\end{array}$ \\
\hline
\end{tabular}


Table 9.1 (Continued)

\begin{tabular}{|c|c|c|}
\hline Category & $\begin{array}{l}\text { Chemical } \\
\text { compounds }\end{array}$ & Working conditions and effects \\
\hline & RSC133 & $\begin{array}{l}\text { Enhances reprogramming efficiency and kinetics of } \\
\text { mouse and human fibroblasts transduced with } \\
\text { OSKM [79] }\end{array}$ \\
\hline \multirow[t]{6}{*}{ TGF $\beta$ inhibitor } & E616451 & $\begin{array}{l}\text { Replaces Sox2 to induce iPSCs from OKM-transduced } \\
\text { MEFs only in the presence of VPA [81] }\end{array}$ \\
\hline & E616452 (RepSox) & $\begin{array}{l}\text { Functionally substitutes transgenic Sox } 2 \text { and alleviates } \\
\text { the need for c-Myc for reprogramming in MEFs [81, } \\
\text { 82] }\end{array}$ \\
\hline & SB431542 & $\begin{array}{l}\text { Replaces Sox2 during reprogramming of MEFs [81] } \\
\text { Increases human fibroblast reprogramming with } \\
\text { OSKM when combined with PD0325901 [86] }\end{array}$ \\
\hline & A-83-1 & $\begin{array}{l}\text { Enhances human keratinocyte reprogramming when } \\
\text { combined with PD0325901 [106] }\end{array}$ \\
\hline & & $\begin{array}{l}\text { Improves ultimate reprogramming and maintenance o } \\
\text { iPSCs generated from rat-liver epithelial cells } \\
\text { transduced with OSK when combined with } \\
\text { PD0325901 and CHIR99021 [85] }\end{array}$ \\
\hline & & $\begin{array}{l}\text { Enables reprogramming of MEFs transduced with Oct } 4 \\
\text { alone when combined with a protein arginine } \\
\text { methyltransferase inhibitor, AMI-5; the iPSCs can } \\
\text { give rise to liveborn pups through tetraploid } \\
\text { complementation [87] }\end{array}$ \\
\hline Wnt signaling & Wnt3a & $\begin{array}{l}\text { Wnt3a-conditioned medium significantly promotes } \\
\text { reprogramming of MEFs transduced with OSK, but } \\
\text { not OSKM [94] }\end{array}$ \\
\hline \multirow[t]{4}{*}{ GSK3 inhibitor } & CHIR99021 & $\begin{array}{l}\text { Promotes "pre-iPSCs" into fully reprogrammed cells } \\
\text { under LIF treatment when combined with } \\
\text { PD0325901 [97] }\end{array}$ \\
\hline & & $\begin{array}{l}\text { Enables reprogramming of MEFs transduced with OK } \\
\text { [74] }\end{array}$ \\
\hline & & $\begin{array}{l}\text { Enables reprogramming of human keratinocytes } \\
\text { transduced with OK when combined with Parnate } \\
\text { [74] }\end{array}$ \\
\hline & Kenpaullone & $\begin{array}{l}\text { Induces iPSC generation from MEFs transduced with } \\
\text { OSM [98] }\end{array}$ \\
\hline \multirow[t]{2}{*}{ Rock inhibitor } & Y27632 & $\begin{array}{l}\text { Widely used in hiPSC generation, improving cell } \\
\text { survival and colony formation }\end{array}$ \\
\hline & Thiazovivin & $\begin{array}{l}\text { Promotes reprogramming when combined with } \\
\text { SB431542 and PD0325901 [86] }\end{array}$ \\
\hline $\begin{array}{l}\text { Aurora kinase } \\
\text { inhibitor }\end{array}$ & BIM0086660 & $\begin{array}{l}\text { Enhances reprogramming of MEFs transduced with } \\
\text { OSKM [102] }\end{array}$ \\
\hline \multirow[t]{2}{*}{$\begin{array}{l}\text { Src kinase } \\
\quad \text { inhibitor }\end{array}$} & $\mathrm{El} 275$ & $\begin{array}{l}\text { Enables reprogramming of MEFs transduced with } \\
\text { OKM when combined with VPA [81] }\end{array}$ \\
\hline & $\begin{array}{l}\text { Dasatinib, PP1, } \\
\text { and iPYrazine }\end{array}$ & $\begin{array}{l}\text { Sufficient to support reprogramming of MEFs in the } \\
\text { absence of exogenous Sox2 [104] }\end{array}$ \\
\hline
\end{tabular}


Table 9.1 (Continued)

\begin{tabular}{|c|c|c|}
\hline Category & $\begin{array}{l}\text { Chemical } \\
\text { compounds }\end{array}$ & Working conditions and effects \\
\hline MEK inhibitor & PD0325901 & $\begin{array}{l}\text { Promotes transition to mature iPSCs }[55,97] \\
\text { Enhances hiPSCs generation when combined with } \\
\text { TGF } \beta \text { inhibitors }[86]\end{array}$ \\
\hline $\begin{array}{l}\text { L-calcium- } \\
\text { channel } \\
\text { agonist }\end{array}$ & BayK8644 & $\begin{array}{l}\text { Improves reprogramming of MEFs transduced with OK } \\
\text { when combined with BIX01294 and RG108 [69] }\end{array}$ \\
\hline PKA activator & 8-Br-cAMP & $\begin{array}{l}\text { Improves reprogramming efficiency of human } \\
\text { neonatal foreskin fibroblasts [105] }\end{array}$ \\
\hline PDK1 activator & PS48 & $\begin{array}{l}\text { Promotes iPSC generation from human somatic cells } \\
\text { transduced with only Oct } 4 \text { when combined with } \\
\mathrm{NaB} \text { and A-83-01 [106] }\end{array}$ \\
\hline $\begin{array}{l}\text { E-cadherin } \\
\text { inducer }\end{array}$ & $\begin{array}{l}\text { Apigenin and } \\
\text { Luteolin }\end{array}$ & Enhance mouse iPSC generation [100] \\
\hline \multirow{3}{*}{$\begin{array}{l}\text { Senescence } \\
\text { alleviator }\end{array}$} & Resveratrol & Improves reprogramming efficiency [142] \\
\hline & Vitamin C & $\begin{array}{l}\text { Improves reprogramming efficiency of mouse and } \\
\text { human fibroblasts }[76,110]\end{array}$ \\
\hline & & $\begin{array}{l}\text { Improves quality of iPSCs reprogrammed from mouse } \\
\text { fibroblasts and B lymphocytes, allowing generation } \\
\text { of all-iPSC mice [122] }\end{array}$ \\
\hline
\end{tabular}

O, Oct4; K, Klf4; S, Sox2; M, c-Myc.

\subsection{Chemicals Modulating Epigenetic Barriers}

Reprogramming involves the genome-wide reshuffling of the transcriptome and chromatin statuses of a somatic cell to that of a pluripotent cell. At the epigenetic level, this event is reflected in the dynamic and opposite remodeling of histone marks and DNA methylation patterns present in the regulatory regions of somatic- and pluripotent-specific genes [57]. Under physiological conditions, the capacity of transcription factors to bind their target sequences is modulated by the accessibility of DNA, resulting from chromatin organization. The nucleosome, the basic subunit of eukaryotic chromatin, is composed of two molecules of each of the core histone proteins $\mathrm{H} 2 \mathrm{~A}, \mathrm{H} 2 \mathrm{~B}, \mathrm{H} 3$, and $\mathrm{H} 4$, around which DNA is wrapped. DNA accessibility is regulated at different levels by the deposition of specific histone marks on nucleosomes, the inclusion of different histone variants, the activity of ATP-dependent chromatin remodeling factors, and DNA methylation status [58].

A major mechanism controlling chromatin organization is the covalent modification of histones, the building blocks of nucleosomes. Specific enzymes such as histone acetyltransferases, histone deacetylases (HDACs), histone methyltransferases, and histone kinases can target different residues of histones and modulate their function. At the DNA level, the methylation of $\mathrm{CpG}$ islands found in the promoter regions of many genes has profound effects on their transcriptional status. Thus, chromatin-modifying enzymes play critical roles in both reactivating silenced loci and reinstating the closed domains of heterochromatin 
during the global epigenetic remodeling of differentiated cells to pluripotency, suggesting that these enzymes may act as facilitators or barriers to cell-fate transitions [59]. Therefore, by modulating these specific epigenetic barriers, one may promote reprogramming efficiency or even reduce the number of reprogramming factors. For example, inhibition of the H3K79 histone methyltransferase Dot1L by gene knockdown can accelerate reprogramming, significantly increase the yield of iPSC colonies, and substitute for Klf4 and c-Myc [59]. In principle, small molecules specifically modulating these different aspects of chromatin and DNA structure may also have significant effects on reprogramming. Indeed, a number of studies have shown that inhibitors of HDACs, histone methyltransferases, and histone demethylases or inhibitors of DNA methyltransferases can increase reprogramming efficiency and/or replace the activity of certain reprogramming factors $[54,60]$.

\subsubsection{Histone Deacetylase Inhibitors}

Although HDAC inhibitors have shown only a modest effect on the success rate of SCNT (two- to fivefold) [61,62], they have proved to significantly increase the efficiency of iPSC generation. Using mouse embryonic fibroblasts (MEFs) derived from Oct4-GFP reporter mice, Huangfu et al. [54] first showed that three HDAC inhibitors, valproic acid (VPA), trichostatin A (TSA), and suberoylanilide hydroxamic acid (SAHA) greatly improve reprogramming efficiency. Of the three, VPA exerts the most significant effect.

VPA $(2 \mathrm{mM})$ induced $>10 \%$ Oct4-GFP ${ }^{+}$cells in MEFs infected with four-factor (Oct4, Sox2, Klf4, and c-Myc) in 1 week, which amounts to $>100$-fold improvement compared to the control. Furthermore, VPA promoted reprogramming efficiency in a dose-dependent manner and its EC50 was 1.9 mM [54]. VPA has a synergistic effect with hypoxia when reprogramming. Yoshida et al. reported that hypoxia and VPA alone could enhance iPSC generation in MEFs transduced with four factors by $~ 40$-fold and 50-fold respectively, while a combination of VPA and hypoxia could increase the reprogramming efficiency more than 200-fold [63]. Notably, VPA can also increase the percentage of Oct4-GFP ${ }^{+}$ cells by 50 times when using only three factors (Oct4, Sox2, and Klf4), which is still much higher than the four-factor reprogramming without chemical treatment [54]. Further study has shown that VPA can also improve both four- and three-factor reprogramming efficiency by 10-20-fold when used in primary human fibroblasts [64, 65]. Importantly, VPA enables reprogramming of human fibroblasts with only two factors, Oct4 and Sox2, without the need for the oncogenes Klf4 or c-Myc [64]. The two-factor reprogramming efficiency with VPA treatment is comparable to that of three-factor reprogramming without chemical treatment in human fibroblasts [39,64]. Microarray analysis shows that VPA preferentially upregulates ESC-specific genes and downregulates MEF-specific genes in the uninfected MEFs, although VPA treatment alone is not sufficient to generate iPSCs from MEFs [54]. Mechanistically, VPA promotes histone acetylation, and appears to induce in MEFs a global transcriptional program that leans toward an ESC-like state.

Besides its enhancing role in reprogramming, VPA has also been suggested to promote complete reprogramming and facilitate expansion of fully reprogrammed iPSCs by inhibiting the growth of nonreprogrammed cells $[48,66]$. Addition of VPA in four-factor reprogramming generates more homogeneous iPSC colonies that highly resemble ESC colonies [66]. Furthermore, some of these iPSC lines can produce adult mice using tetraploid 
complementation assays, suggesting that fully pluripotent iPSCs can be generated by combination of VPA with four factors [66].

In addition to reprogramming using the four Yamanaka factors, VPA also promotes reprogramming using cell-permeable recombinant proteins or microRNAs. Zhou et al. showed that VPA is essential to the generation of stable iPSCs from MEFs using recombinant proteins (Oct4-11R, Sox2-11R, Klf4-11R, and c-Myc-11R), a method that circumvents genetic modifications associated with the classic approach, which introduces reprogramming genes through retroviral infections [50]. Three $\mathrm{GFP}^{+}$colonies per $5 \times 10^{4} \mathrm{MEF}$ cells were obtained when they were transduced with four proteins (Oct4-11R, Sox2-11R, Klf4-11R, and c-Myc-11R) and treated with VPA, and one GFP ${ }^{+}$colony per $5 \times 10^{4}$ cells when they were transduced with only three proteins (Oct4-11R, Sox2-11R, and Klf4-11R) and treated with VPA. However, no stable $\mathrm{GFP}^{+}$colonies were obtained from transduction of the three or four reprogramming proteins without VPA for the same period of time [50]. Anokye-Danso et al. showed that combination of VPA treatment and miR302/367 cluster expression can generate iPSCs from MEFs without using any exogenous transcription factors, while miR302/367 alone cannot efficiently reprogram MEFs [52]. Further experiments demonstrated that VPA induced degradation of Hdac2 proteins and low levels of Hdac2 permitted miR302/367 reprogramming. However, using human foreskin and dermal fibroblasts, miR302/367 expression generates iPSCs without VPA, presumably due to low levels of Hdac2 being expressed in human fibroblasts [52].

Sodium butyrate $(\mathrm{NaB})$, a naturally occurring fatty acid commonly used as a nutritional supplement and differentiation agent and an analog of VPA, has also been shown to greatly enhance the efficiency of iPSCs from human adult or fetal fibroblasts [65]. After transient butyrate treatment, the iPSC derivation efficiency is enhanced by 15-51-fold. Butyrate has a stronger effect (100-200-fold) on reprogramming in the absence of either Klf4 or c-Myc transgene. BIX01294 (see Section 9.2.2) can synergize with butyrate to further enhance the butyrate-stimulated reprogramming by three factors in human fibroblasts or mesenchymal stem cells (MSCs). By day 6-12 during reprogramming, butyrate treatment enhances histone $\mathrm{H} 3$ acetylation, promoter DNA demethylation, and the expression of endogenous pluripotency-associated genes [65]. Since NaB is an analog of VPA, their reprogramming-enhancing mechanisms might be the same.

Histone acetylation and deacetylation regulate chromatin structure and transcriptional activity. The genome-wide acetylation induced by VPA and other HDAC inhibitors could allow fibroblasts to adopt a more open chromatin structure that facilitates the binding of ectopically expressed reprogramming factors or downstream secondary factors [67], and thus promotes reprogramming efficiency. Since $\mathrm{NaB}$ and VPA appear to have stronger effects on reprogramming than other HDAC inhibitors, it is possible that these two compounds also act through additional, yet unidentified mechanisms to regulate reprogramming.

\subsubsection{Histone Methyltransferase Inhibitor and Demethylase Inhibitor}

Histone methylation and demethylation also play important roles in regulating gene expression and reprogramming. BIX01294 (BIX), an inhibitor of the G9a histone methyltransferase [68], improves the reprogramming efficiency in mouse neural stem cells (NSCs) and fibroblasts that were transduced with only two factors $(\mathrm{OK})[55,69]$. The two-factor (OK) reprogramming efficiency of BIX-treated NSCs is comparable to four-factor (OSKM) 
reprogramming [55]. Although the overall efficiency is improved, the reprogramming process is not significantly shortened by the presence of BIX. Interestingly, BIX treatment enables reprogramming of NSCs to iPSCs with KSM, without the need for Oct4, albeit at a low frequency [55]. Because G9a promotes histone H3 methylation on Lys 9 and inhibits Oct4 by attracting de novo DNA methylases to the Oct4 promoter [70], by inhibiting G9a, BIX may release repression of endogenous Oct4 and thus reduce the need for exogenous Oct4 during reprogramming of NSCs. It has been shown that Sox 2 can maintain ESC pluripotency by regulating the expression of Oct4 [71]. NSCs endogenously express Sox 2 and c-Myc, and expression levels of Sox 2 and c-Myc are comparable to those in ESCs [72,73]. Generation of iPSCs from NSCs can be achieved by expressing only Oct4 and Klf4 [73]. Therefore, it is not clear whether BIX actually functionally replaced Sox 2 and c-Myc in the cases of reprogramming of NSCs. In a further study using OK-infected MEFs, Shi et al. showed that, unlike NSCs, BIX treatment did not effectively enhance generation of iPSCs [69]. However, when combined with a DNMT inhibitor, RG108, and an L-calcium channel agonist, BayK8644, BIX significantly increased reprogramming of MEFs in the absence of Sox 2 and c-Myc [69]. The mechanism by which BayK8644 acts remains unclear, however, as BayK8644 alone or in combination with BIX did not alter cell proliferation or Sox 2 expression.

In a reprogramming study using human primary keratinocytes, the cells were transduced with two-factor combinations (Oct4/Klf4, Oct4/Sox2, and Sox2/Klf4) and treated with CHIR99021 (a glycogen synthase kinase 3 (GSK3) inhibitor) alone or combined with epigenetic modifiers, including inhibitors of DNA methyltransferase (DNMT) (RG108), histone methyltransferase (BIX01294), HDAC (VPA), and lysine-specific demethylase 1 (LSD 1) (Parnate). Using the human pluripotency cell-surface marker Tra-1-81, hESClike colonies were identified only from cells infected by Oct4 and Klf4 and treated with CHIR99021 and parnate [74]. Parnate, also known as tranylcypromine, is a monoamine oxidase inhibitor. It exhibits a potent inhibitory effect on LSD1 and inhibition of H3K4 demethylation, but it does not affect the acetylation of H3K9/K14. Parnate may facilitate the reprogramming of human keratinocytes by inhibiting H3K4 demethylation. In another study, parnate together with VPA, CHIR99021, and E-616452 (a transforming growth factor $\beta$ (TGF $\beta$ ) inhibitor) was sufficient to induce reprogramming in MEFs in combination with a single transcription factor, Oct4, without the need for Sox2, Klf4, or c-Myc [75]. However, how histone demethylase inhibitors enhance reprogramming remains unclear.

Chemical inhibitors of histone demethlyases (the iron chelator desferrioxamine or the $\alpha$-ketoglutarate analog dimethyloxalylglycine) inhibit Sox $2 / K l f 4 / O c t 4$ (SKO) reprogramming in a dose-dependent manner [76]. The combination of the histone demethylases Jhdm1a and $1 \mathrm{~b}$ can promote reprogramming of MEFs using three-factor SKO, and even more efficiently in the presence of vitamin $C$ [76]. In addition, through its histone demethylase activity, Jhdm $1 \mathrm{~b}$ enables reprogramming with only Oct4 in the presence of vitamin C [76].

In a recent study, a new class of chemical compounds that specifically inhibit histone demethylase LSD1 has been developed [77]. Unlike monoamine oxidase inhibitors, the new LSD1 inhibitors specifically interact with LSD1 and inhibit its activity without forming a covalent bond. These new inhibitors enhance H3K4 methylation and derepress epigenetically suppressed genes in vivo. Strikingly, these compounds inhibited the proliferation of pluripotent cancer cells (including teratocarcinoma, embryonic carcinoma, and seminoma) 
and ESCs that expressed the stem-cell markers Oct4 and Sox 2 while displaying minimum growth-inhibitory effects on nonpluripotent cancer or normal somatic cells [77]. It would be interesting to see whether these new LSD1 inhibitors have a different effect on cellular reprogramming to pluripotency compared with parnate, and such studies might be able to provide further mechanistic understanding of reprogramming.

\subsubsection{DNA Methyltransferase Inhibitors}

DNA methylation is one of the common epigenetic mechanisms regulating gene silencing. Pluripotent stem cells have a less methylated epigenetic signature than somatic cells. Therefore, inhibition of DNMTs can reactivate the expression of genes that have been repressed by DNA methylation, which may facilitate cellular reprogramming.

A prototypical inhibitor of DNMT, 5-Aza-cytidine (5-aza), is a chemical analog of the DNA and RNA nucleoside cytidine. 5-aza needs to be incorporated into DNA to covalently trap DNMTs [78]. 5-aza has a half-life of 17 hours and is considerably cytotoxic [78]. Not only can it enhance reprogramming efficiency, but it also improves reprogramming quality by pushing partially reprogrammed cells forward to a complete reprogrammed state. 5-aza treatment increases the percentage of Oct4-GFP ${ }^{+}$cells in both four- and three-factorinfected MEFs in a dose-dependent manner, and increases the number of ESC-like colonies formed during reprogramming [24,54]. Dexamethasone $(1 \mu \mathrm{M})$, a synthetic glucocorticoid, improves the effect of 5-aza further, although dexamethasone alone has no effect [54]. Furthermore, 5-aza induces a rapid and stable transition to a fully reprogrammed iPSC state when used on partially reprogrammed MEFs [24]. Partially reprogrammed MEFs treated with 5-aza reactivated endogenous Oct4, exhibited demethylation at the promoters of pluripotency genes, and formed teratomas when injected into severe combined immunodeficiency (SCID) mice, just like fully reprogrammed iPSCs [24]. These findings suggest that DNA demethylation is a critical step in direct reprogramming, and that inhibition of DNMT facilitates the transition to pluripotency by lowering this epigenetic barrier [24].

RG108 is a non-nucleoside DNMT inhibitor with a mean half-life of 20 days [78]. It significantly inhibits DNA methylation in cells at $10 \mu \mathrm{M}$ without detectable toxicity, distinguishing it from nucleoside-based inhibitors like 5-aza. Furthermore, RG108 can inhibit free DNMTs; the inhibition of DNMT activity is achieved by blocking the enzyme active site [78]. RG108 enhances the reprogramming efficiency of MEFs transduced with Oct4 and Klf4 in the presence of a histone methyltransferase inhibitor, BIX01294 [69]. It also increases the reprogramming efficiency of human fibroblasts transduced with all four Yamanaka factors (OSKM) by twofold [65]. Although RG108 is a more potent and noncovalent DNMT inhibitor than 5-aza, it appears to have a lesser effect on reprogramming. This could be due to the differences in experimental specifications in different studies. The mechanism by which RG108 enhances reprogramming has not been further elucidated, though it is reasonable to assume that RG108 and 5-aza both promote reprogramming by modulating DNA methylation.

More recently, Lee et al. reported that a novel indoleacrylic acid analog, named RSC133, promotes iPSC generation from both mouse and human fibroblasts [79]. Continuous treatment with RSC133 throughout reprogramming increased the reprogramming efficiency by up to fourfold. Under hypoxic conditions $\left(5 \% \mathrm{O}_{2}\right)$, RSC133 synergistically increased 
the reprogramming efficiency to five- to sixfold compared to untreated controls [79]. On treatment with RSC133, OSKM-transduced human fibroblasts displayed a rapid activation of pluripotency genes and highly proliferative hESC-like colonies in 10 days, suggesting that RSC133 significantly enhanced both the efficiency and kinetics of the reprogramming process [79]. The enhanced reprogramming efficiency was most likely due to RSC133 promotion of cell proliferation, ablation of pro-senescence phenotypes, inhibition of DNMT1 activity, and subsequent reduction of HDAC activity [79].

5-aza, RG108, and RSC133 are structurally very different DNMT inhibitors, and the ways in which they modulate DNMT activities are also different. It is not clear whether these differences have any effects in their promotion of reprogramming.

\subsection{Chemicals Targeting Signaling Pathways}

Although chemicals that modify epigenetic state have been shown to improve reprogramming efficiency, the precise molecular mechanisms remain elusive. Presumably, they facilitate the shifting of epigenetic balances among different states during reprogramming and these epigenetic modifiers have broad effects on the epigenome [80]. Studies have shown that small molecules targeting certain signaling pathways can also promote reprogramming. Many of these signaling pathways are involved in the regulation of pluripotency gene expression, cell proliferation, and pluripotency maintenance. Therefore, these studies not only show that chemical compounds that modulate signaling pathways can improve generation of iPSCs but also provide insights and direct clues about the molecular mechanisms of reprogramming.

\subsubsection{TGF $\beta$ Signaling Inhibitors}

It has been shown that TGF $\beta$ signaling plays important roles in reprogramming. In a study by Ichida et al. through a high-content chemical screen, two inhibitors of TGF $\beta$ receptor-1 kinase (E616451 and E616452) had been identified [81]. E616451 can replace Sox 2 to induce iPSCs from OKM-transduced MEFs in the presence of VPA. In contrast, E616452 can functionally replace transgenic Sox2 in the absence of VPA and c-Myc, and thus it has been named "RepSox" [81]. RepSox is not involved in direct activation of endogenous Sox2. Similarly, TGF $\beta$-neutralizing antibodies and a nonspecific inhibitor of TGF $\beta$ receptor-1 kinase (SB431542) can also replace Sox2 during reprogramming. Furthermore, inhibition of TGF $\beta$ signaling early in reprogramming also alleviates the need for transgenic c-Myc expression [81, 82]. Maherali and Hochedlinger demonstrated that inhibition of TGF $\beta$ signaling at early time points (days 1-3) during mouse fibroblast reprogramming could effectively substitute for transgenic Sox2 [82]. In contrast, Ichida et al. found that Sox 2 replacement by RepSox was a late event (beginning at day 10-11). Moreover, only a 24-hour pulse of RepSox at day 11 on OKM-transduced MEFs was able to induce detectable reprogramming, suggesting that RepSox acts by triggering an endogenous switch in partially reprogrammed cells [81,83]. In OKM-transduced partially reprogrammed MEFs, inhibition of TGF $\beta$ signaling by RepSox, SB431542, and anti-TGF $\beta$ antibodies can induce Nanog expression, leading to full reprogramming [81]. However, the mechanism by which RepSox activates Nanog remains elusive. 
Several TGF $\beta$ superfamily members represent important contributors to the pluripotent state. For instance, BMP4 signaling helps maintain mouse ESCs in an undifferentiated state [84], and use of a TGF $\beta$ inhibitor (A-83-01) in conjunction with mitogen-activated protein kinase kinase (MEK) and GSK3 inhibitors is critical for ultimate reprogramming and maintenance of rat iPSCs [85]. Lin et al. found that reprogramming efficiency was significantly improved in OSKM-transduced human primary fibroblasts when treated with a combination of the ALK5 inhibitor SB431542 $(2 \mu \mathrm{M})$ and the MEK inhibitor PD0325901 [86]. However, Maherali and Hochedlinger did not observe enhancing effects of RepSox in human fibroblasts transduced with OSKM [82]. The TGF $\beta$ inhibitor A-83-01, combined with a protein arginine methyltransferase inhibitor, AMI5, enabled reprogramming of MEFs transduced with Oct4 alone, and the derived iPSCs could give rise to liveborn pups through tetraploid complementation [87].

Reprogramming of fibroblasts to iPSCs requires a fundamental process termed mesenchymal-epithelial transition (MET) [88, 89]. During MET, the mesenchymal fibroblasts undergo dramatic morphological changes that result in iPSCs with distinct cell polarities, boundaries, and cell-cell interactions, as well as high E-cadherin expression [80]. Inhibition of TGF $\beta$ signaling promotes MET during reprogramming [88, 89]. Therefore, those TGF $\beta$ inhibitors that enhance reprogramming reported previously might also involve enhanced MET induced by the inhibitors $[81,82,86]$.

\subsubsection{Wnt Signaling and GSK3 Inhibitors}

The Wnt signaling and $\beta$-catenin pathways have been shown to play important roles in both ESC self-renewal and ESC differentiation toward lineage-committed cell types [90,91]. The Wnt signaling pathway contributes to the maintenance of self-renewal of both mouse embryonic stem cells (mESCs) and hESCs through inhibition of GSK3 and the subsequent nuclear accumulation of $\beta$-catenin [91,92]. Mechanistically, when the canonical Wnt signaling pathway is stimulated, $\beta$-catenin translocates into the nucleus and then interacts with Tcf/Lef proteins to activate target genes [93]. During reprogramming, constitutively active $\beta$-catenin may promote reprogramming of fibroblasts to pluripotency [21]. When Oct4-GFP reporter MEFs were infected with three factors (OSK), a small population $(\sim 0.3 \%)$ of $\mathrm{GFP}^{+}$ cells was detected until day 20 only when cultured in Wnt3a-conditioned medium (Wnt3aCM) [94]. The iPSC colonies induced by OSK + Wnt3a-CM morphologically appeared homogenously ESC-like. Moreover, at 4 weeks, Wnt3a-CM promoted the reprogramming efficiency up to 20-fold in OSK-infected MEFs based on endogenous Oct4-GFP ${ }^{+}$colony counting. Conditioned media from control fibroblasts also moderately improved reprogramming, suggesting that normal fibroblasts may secrete soluble factors beneficial to reprogramming [94]. In contrast, Wnt3a-CM only slightly increased reprogramming efficiency when using OSKM four factors (including c-Myc) [94]. These data suggest that Wnt3a can compensate for exogenous c-Myc gene expression in terms of reprogramming efficiency.

Besides the direct promoting effects on reprogramming, Wnt3a can also enhance somaticcell reprogramming mediated by cell fusion [95]. Deletion of Tcf3, which in turn derepresses $\beta$-catenin target genes, can enhance both cell fusion-mediated and Oct4-Klf4 (OK)-induced direct reprogramming of NSCs [96]. Interestingly, this reprogramming process appears not to be independent of $\beta$-catenin; rather, it seems to be coupled with the activity of the Wnt 
signaling pathway [96]. Furthermore, the increased reprogramming efficiency is largely attributable to genome-wide epigenome modifications that occur before the endogenous stem-cell genes are reactivated in the iPSC clones.

GSK3 is an important part of the canonical $\beta$-catenin/Wnt pathway, which signals the cell to divide and proliferate. Inhibition of GSK3 has been shown to enhance reprogramming, presumably due to activation of Wnt signaling by inhibition of GSK3. Silva et al. showed that inhibition of mitogen-activated protein kinase kinase (MEK) and GSK3 (using PD0325901 and CHIR99021 (2i), respectively) can promote "pre-iPSCs" into fully reprogrammed pluripotent cells (ground-state pluripotency) under leukemia inhibitory factor (LIF) treatment [97]. Another GSK3/cyclin-dependent kinase 2 (CDK2) inhibitor, kenpaullone, can substitute Klf4 in reprogramming of MEFs in the presence of Oct4, Sox2, and cMyc [98]. However, as a more specific GSK3 inhibitor, CHIR99021, failed in producing the same positive effects on inducing the reprogramming of MEF cells under the Oct4/Sox 2/c-Myc transduction [98], the effects of kenpaullone may not result from its GSK3 inhibition, and the precise mechanism remains elusive.

The GSK3 inhibitor CHIR99021 $(10 \mu \mathrm{M})$ can however induce the reprogramming of MEFs transduced by only two factors, Oct4 and Klf4, but not any other two-factor combination of OSKM [74]. MEFs do not express Sox 2 endogenously, and RT-qPCR analysis revealed that CHIR99021 treatment did not induce the expression of Sox2 and Oct4 in MEFs [74]. Therefore, the mechanisms by which CHIR99021 promotes the reprogramming of MEFs transduced by Oct 4 and Klf4 are independent of direct Sox 2 induction. When combined with parnate (known as tranylcypromine, $2 \mu \mathrm{M}$ ), an inhibitor of lysine-specific demethylase 1 (LSD1), CHIR99021 can cause the reprogramming of human primary keratinocyte transduced with the two factors Oct4 and Klf4 (OK) [74]. On average, about two Tra-1-81-positive colonies can be obtained from $1 \times 10^{5}$ transduced keratinocytes. Addition of PD0325901 $(0.5 \mu \mathrm{M})$ and SB431542 $(2 \mu \mathrm{M})$ can further increase the reprogramming efficiency using CHIR99021 and parnate with OK [74]. Notably, pretreatment of mESCs with a small-molecule GSK3 $\beta$ kinase inhibitor, 6-bromoindirubin-3'-oxime (BIO), can enhance the ability of ESCs to reprogram somatic cells after fusion [95]. However, BIO cannot enhance somatic-cell reprogramming to pluripotency via direct reprogramming induced by OSKM [95].

\subsubsection{Other Kinase Inhibitors and Activators}

An inhibitor of the protein kinase ROCK, known as Y27632, which allows survival and clonogenicity of single dissociated hESCs [99], has been widely used in hiPSC generation. Another ROCK inhibitor, thiazovivin, whose function is to regulate E-cadherin stability and facilitate cell-cell interactions, can also promote reprogramming when combined with SB431542 and PD0325901 [86]. Another study shows that two small molecules, apigenin and luteolin, can also enhance mouse induced pluripotent stem cell (miPSC) generation through upregulation of E-cadherin [100]. A myosin inhibitor, blebbistatin, can efficiently inhibit hiPSC apoptosis induced by ROCK-dependent hyperactivation of actomyosin during dissociation and replating, resulting in better cell survival and colony formation [101]. However, it has not been shown whether blebbistatin can also enhance reprogramming efficiency.

Inhibitors for other kinases such as p38 MAPK, inositol trisphosphate 3-kinase (IP3K), and Aurora A kinase (Aurka) can also enhance iPSC generation from MEFs. Inhibition 
of Aurka can inactivate GSK3 $\beta$ mediated by the Akt signaling pathway, resulting in an increased reprogramming efficiency [102]. Contradictorily, Lee et al. reported that during reprogramming, Aurka activity increases, while depletion of Aurka leads to increased activity of $\mathrm{p53}$, resulting in a reduced reprogramming efficiency [103].

A few other small molecules that affect many other signaling pathways also facilitate iPSC reprogramming. Pan-Src-family kinase inhibitors, including EI275, Dasatinib, PP1, and iPYrazine, support reprogramming of MEFs in the absence of exogenous Sox 2 as efficiently as TGF $\beta$ inhibitors [81, 104]. An activator of cyclic AMP-dependent protein kinase (PKA), 8-bromoadenosine 3', 5'-cyclic monophosphate (8-Br-cAMP), can improve the reprogramming efficiency of human neonatal foreskin fibroblasts [105].

These observations are consistent with the fact that the GSK3 pathway, the PKA pathway, and Src-family kinases influence ESC self-renewal and differentiation. These data again suggest that the inhibitors and activators of key signaling pathways in ESC self-renewal and differentiation are more likely to be able to regulate the efficiency of reprogramming to pluripotency. This can be used as one of the guidelines for small-scale chemical screen.

Interestingly, an activator of the 3-phosphoinositide-dependent protein kinase-1 (PDK1)PI3K/Akt pathway, PS48, can significantly induce expression of glycolytic genes and promote iPSC generation from human somatic cells transduced with only Oct 4 when combined with $\mathrm{NaB}$ and A-83-01 [106]. These data suggest that modulation of cellular metabolism by small molecules may provide an additional way of improving reprogramming.

\subsubsection{Cell Senescence Alleviators}

Cell senescence represents an irreversible arrest in the G1 phase of the cell cycle induced by replicative exhaustion or in response to a number of stresses, such as DNA damage, chemotherapeutic drugs, or ectopic expression of oncogenes. This arrest is mainly implemented through p53 activation and upregulation of the CDK inhibitors p16 ${ }^{\mathrm{INK} 4 \mathrm{a}}$ and $\mathrm{p} 21^{\mathrm{CIP} 1}$ [107]. The observation that early-passage MEFs reprogram with much higher efficiency than late passages, in which $\beta$-galactosidase-positive senescent cells accumulate, suggests a high replicative potential of the donor cell is critical for successful reprogramming to pluripotency [108]. Moreover, ectopic expression of OKSM in human fibroblasts is sufficient to increase the percentage of G1-arrested cells displaying senescent-associated $\beta$ galactosidase (SA $\beta$-Gal) activity and senescence-associated heterochromatic foci (SAHF) [109].

Several factors may contribute to the induction of senescence and/or apoptosis in reprogramming factor-expressing cells. For instance, the level of reactive oxygen species (ROS) $[109,110]$ is significantly increased in the pool of cells expressing OSKM, correlating with an overall upregulation of critical senescent effectors such as p16 ${ }^{\mathrm{INK} 4 \mathrm{a}}$ and $\mathrm{p} 21^{\mathrm{CIP}}$ [109]. Alternatively, but not exclusively, senescence and/or apoptosis can be elicited by an increase in the DNA double-strand breaks (DSBs) observed in cells expressing OSKM or OSK [111]. Consistently, genetic ablation or shRNA knockdown of the tumor suppressors p53 [109, 112-115], p2 ${ }^{\text {CIP1 }}[109,113]$, or genes belonging to the Ink4/Arf locus $[108,109,116]$ have a positive effect on the efficiency of reprogramming, suggesting the activity of these genes acts as a barrier to reprogramming.

Therefore, transient alleviation of cell senescence using chemical compounds during reprogramming may represent a simple way of increasing reprogramming efficiency, particularly in the context of nonintegrative approaches, which usually show very low 
efficiencies [117]. However, because p53, p16 $6^{\mathrm{INK} 4 \mathrm{a}}$, and $\mathrm{p} 21^{\mathrm{CIP}}$ are key players in safeguarding chromosomal stability, their manipulation should be considered very carefully in order to generate therapeutically relevant iPSC lines, since their activity during reprogramming may be essential to eliminating cells accumulating chromosomal aberrations due to high levels of DNA damage [111]. A more appealing alternative would therefore be to act upstream, on the causes leading to cell senescence during reprogramming.

As we just mentioned, one of the possible triggers of cell senescence and/or apoptosis during reprogramming is the accumulation of ROS. High ROS levels promote the modification of individual nucleotide bases, the formation of single and double strand breaks [118], and telomere shortening [119], all of which result in the activation of the p53 pathway. Consistently, a recent study shows that two antioxydants, resveratrol and curcumin, significantly improve reprogramming efficiency in MEFs [120]. Another antioxidant, vitamin $\mathrm{C}$, has also been shown to positively affect the reprogramming of MEFs using OSKM and OSK, and of human fibroblasts using OSKM [110]. Reprogramming enhancement by vitamin $\mathrm{C}$ does not however seem to result from its antioxidant activity but rather from the decreasing level of p53 [110]. Further studies have revealed that vitamin C enhances reprogramming by promoting histone demethylation of the Ink/Arf locus of Jhdm $1 \mathrm{a} / \mathrm{lb}$ and by activating the miR302-367 microRNA cluster [76]. Vitamin $\mathrm{C}$ has also been shown to influence DNA methylation patterns in hESCs [121]. Reprogramming in the presence vitamin $\mathrm{C}$ reduces the hypermethylation of the Dlk1-Dio3 locus by promoting a chromatin configuration, interfering with the binding of the de novo DNMT Dnmt3a and thus allowing all-iPSC mice to be generated from mature B cells [122].

\subsection{Chemicals Promoting Lineage Reprogramming}

In addition to reprogramming to pluripotency, it has been shown that one cell type can be directly reprogrammed into another without going through a pluripotent state by ectopically expressing lineage-specific genes. This is termed lineage reprogramming or transdifferentiation and can be achieved both in vitro and in vivo. For example, fibroblasts can be directly converted into myoblasts by MyoD [123], functional neurons by Ascl1/Brn2/Mytl1 [124], and cardiomyocytes by Gata4/Mef2c/Tbx5 [125], while exocrine cells in a mouse pancreas can be converted into islet $\beta$-like cells by Pdx1/MafA/Ngn3 [126]. It seems that lineage reprogramming may be a faster and more efficient means of generating functional cell types and essentially eliminates the teratoma risk of using pluripotent cells. However, cell proliferation rate may be limited during lineage reprogramming, because terminally differentiated cells usually have a very low proliferation rate compared to iPSCs. Recently, it has been shown that by transiently expressing all four Yamanaka factors or by expressing one of the Yamanaka factors (Sox2 or Oct4) in mouse or human fibroblasts, lineage-specific cells or precursors can be generated directly from fibroblasts without going through pluripotency [127-130]. One of the advantages of this method is that the transcription factors are universal and may be easily replaced by safer and more convenient methods without genetic modification [131].

As in reprogramming to pluripotency, there is a dramatic change in epigenetic state during lineage reprogramming; therefore, it is conceivable that we can improve lineagereprogramming efficiency using chemical molecules as epigenetic modifiers, although this 
remains to be investigated. Furthermore, we can imagine that employing lineage-specific chemical signals during transdifferentiation may facilitate lineage conversion.

Ladewig et al. showed that human fibroblasts can be efficiently converted into neuronal cells by only expressing Ascl1 and Ngn2 in the neuronal medium containing a set of small molecules (GSK3 inhibitor CHIR99021, TGF $\beta$ inhibitor SB-431542, and Noggin) [132]. Spontaneously contracting cardiomyocytes can be generated from mouse fibroblasts that transiently express all four Yamanaka factors for only 4-6 days with a JAK inhibitor (JI1) and without LIF, followed by BMP4 treatment in a chemically defined medium for an additional 5 days [127]. Similarly, mouse fibroblasts can be efficiently converted into NSCs when the transient reprogrammed cells are exposed to neural reprogramming media containing EGF, FGF2, and FGF4 [128]. Furthermore, mouse and human fibroblasts can be converted into multipotent NSCs by only expressing a single factor, Sox2 [129]. During this lineage reprogramming, the fibroblasts are cultured on gelatin-coated glass coverslips in NSC medium supplemented with EGF and FGF2 [129]. Human fibroblasts have also been directly converted to multipotent blood progenitors by prolonged overexpression of Oct4 and treatment with complete F12 media containing FGF2 and IGF-II [130]. Supplementing with Flt3 and stem-cell factor (SCF) further increases the frequency of lineage reprogramming [130].

In addition to the two lineage-reprogramming strategies discussed here, lineage-specific cells can also be efficiently generated from hPSCs by expressing a set of lineage-specific transcription factors at certain stages of differentiation and culturing them with lineageinduction media with specific chemical signals [133-136]. For example, mature white and brown adipocytes can be generated by expressing PPARG2 alone or in combination with CEBPB and/or PRDM16 in mesenchymal progenitor cells derived from hPSCs under adipogenic medium [134]; functional neurons can also be rapidly generated from hPSCs by expressing Ascl1/Brn2/Mytl1 under N3 media [135]. The media used in these studies are the same as the media used for directed differentiation from hPSCs, with the addition of lineage-specific chemical signals.

Since some of the chemicals used for pluripotency reprogramming can decondense chromatin structure and reduce epigenetic barriers for reprogramming, we would imagine that if we select and combine the chemicals used for pluripotency reprogramming together with lineage-specific chemicals, we might be able to further improve lineage conversion efficiency. Chemicals used for pluripotency reprogramming may ease the transition into other lineages, and lineage-specific chemicals may direct conversion toward a desired lineage. This concept remains to be tested at this time.

\subsection{Conclusion}

Pluripotent stem cells represent powerful platforms for basic research, disease modeling, drug screening, and regenerative medicine. The recent availability of simple approaches to the generation of patient-specific pluripotent stem cell lines through pluripotency reprogramming or specific differentiated cell types through lineage reprogramming not only brings us closer to the implementation of cell-based therapies in humans but also contributes significantly to our understanding of the mechanisms underlying lineage commitment and cell plasticity. Efficiently complementing the overexpressing of defined transcription factors 
with chemical manipulation represents an effective tool for directing changes in cell fate and facilitating reprogramming or transdifferentiation, and for dissecting the mechanisms governing these dramatic changes in cell fate.

Chemical compounds may have an effect on reprogramming at different levels by modulating epigenetic states, regulating master gene expression, controlling key signaling pathways, or preventing cell senescence. To improve reprogramming through chemical treatment, future work should focus on screening compounds that improve the erasure of the somatic gene expression signature, activate master regulators of pluripotency and selfrenewal, and enhance chromatin plasticity. Sometimes, the mechanisms by which novel small molecules identified in a chemical screen can enhance reprogramming efficiency or quality remain unknown and need to be further investigated. For example, an Oct4-activating compound was found to activate both Oct 4 and Nanog and increase reprogramming efficiency in MEFs transduced with four factors, but the underlying mechanism was unclear [137]. In addition, it seems that most chemicals can not only promote reprogramming in mouse cells but also enhance reprogramming in human cells. Whether these chemicals function in the same way and result in the same quality of iPSCs in mouse and human remains to be explored. Understanding how chemicals assist in reprogramming will allow us to use them more effectively.

For cell-replacement therapy, high-quality, safe, and large numbers of iPSCs are desired. We need not only to find chemicals that can significantly speed up the generation of iPSCs from a patient's cells but also to identify chemicals that can generate safe iPSCs. It has been shown that iPSCs can still acquire many mutations during reprogramming even when using a nonintegrated method for transduction [111,138-141]. These genetic abnormalities may originate from the oncogenic activity of the reprogramming factors and from epigenetic remodeling during reprogramming. Using chemicals to replace one or more transcription factors has been a step toward achieving safer iPSCs. So far, there have been no chemicals or chemical combinations that can completely replace the four transcription factors reported. Further studies focusing on the combination of multiple chemicals at certain reprogramming stages may be helpful in completely eliminating the need for transcription factors. In addition, most of the chemicals used to enhance reprogramming can promote global modifications and result in genetic aberrations in iPSCs. Identifying additional chemicals for the generation of safe iPSCs is critical to the therapeutic application of iPSCs.

To conclude, the development of chemical approaches to manipulating cell fate and the constant synthesis of new compounds or improvement of existing ones has placed small molecules in a central position in the design of safe patient-derived iPSC lines suitable for cell-replacement therapy. By carefully selecting and combining the chemicals used for pluripotency reprogramming with lineage-specific chemical signals, we should be able to efficiently generate safe and high-quality lineage-specific functional cells for such therapy.

\section{References}

(1) Thomson, J.A., Itskovitz-Eldor, J., Shapiro, S.S. et al. (1998) Embryonic stem cell lines derived from human blastocysts. Science, 282 (5391), 1145-1147.

(2) Zhu, Z. and Huangfu, D. (2013) Human pluripotent stem cells: an emerging model in developmental biology. Development (Cambridge, England), 140 (4), 705-717.

(3) Bradley, J.A., Bolton, E.M., and Pedersen, R.A. (2002) Stem cell medicine encounters the immune system. Nature Reviews Immunology, 2 (11), 859-871. 
(4) Gurdon, J.B. (1962) The developmental capacity of nuclei taken from intestinal epithelium cells of feeding tadpoles. Journal of Embryology and Experimental Morphology, 10, 622-640.

(5) Wilmut, I., Schnieke, A.E., Mcwhir, J. et al. (1997) Viable offspring derived from fetal and adult mammalian cells. Nature, 385 (6619), 810-813.

(6) Kato, Y., Tani, T., Sotomaru, Y. et al. (1998) Eight calves cloned from somatic cells of a single adult. Science, 282 (5396), 2095-2098.

(7) Wakayama, T., Perry, A.C., Zuccotti, M. et al. (1998) Full-term development of mice from enucleated oocytes injected with cumulus cell nuclei. Nature, 394 (6691), 369-374.

(8) Baguisi, A., Behboodi, E., Melican, D.T. et al. (1999) Production of goats by somatic cell nuclear transfer. Nature Biotechnology, 17 (5), 456-461.

(9) Polejaeva, I.A., Chen, S.H., Vaught, T.D. et al. (2000) Cloned pigs produced by nuclear transfer from adult somatic cells. Nature, 407 (6800), 86-90.

(10) Chesné, P., Adenot, P.G., Viglietta, C. et al. (2002) Cloned rabbits produced by nuclear transfer from adult somatic cells. Nature Biotechnology, 20 (4), 366-369.

(11) Shin, T., Kraemer, D., Pryor, J. et al. (2002) A cat cloned by nuclear transplantation. Nature, 415 (6874), 859.

(12) Zhou, Q., Renard, J.P., Le Friec, G. et al. (2003) Generation of fertile cloned rats by regulating oocyte activation. Science, 302 (5648), 1179.

(13) Lee, B.C., Kim, M.K., Jang, G. et al. (2005) Dogs cloned from adult somatic cells. Nature, 436 (7051), 641.

(14) Wakayama, T., Tabar, V., Rodriguez, I. et al. (2001) Differentiation of embryonic stem cell lines generated from adult somatic cells by nuclear transfer. Science, 292 (5517), 740-743.

(15) Wang, L., Duan, E., Sung, L.-Y. et al. (2005) Generation and characterization of pluripotent stem cells from cloned bovine embryos. Biology of Reproduction, 73 (1), 149-155.

(16) Byrne, J.A., Pedersen, D.A., Clepper, L.L. et al. (2007) Producing primate embryonic stem cells by somatic cell nuclear transfer. Nature, $\mathbf{4 5 0}$ (7169), 497-502.

(17) Noggle, S., Fung, H.-L., Gore, A. et al. (2011) Human oocytes reprogram somatic cells to a pluripotent state. Nature, 478 (7367), 70-75.

(18) Tada, M., Tada, T., Lefebvre, L. et al. (1997) Embryonic germ cells induce epigenetic reprogramming of somatic nucleus in hybrid cells. The EMBO Journal, 16 (21), 6510-6520.

(19) Tada, M., Takahama, Y., Abe, K. et al. (2001) Nuclear reprogramming of somatic cells by in vitro hybridization with ES cells. Current Biology, 11 (19), 1553-1558.

(20) Cowan, C.A., Atienza, J., Melton, D.A., and Eggan, K. (2005) Nuclear reprogramming of somatic cells after fusion with human embryonic stem cells. Science, 309 (5739), 1369-1373.

(21) Takahashi, K. and Yamanaka, S. (2006) Induction of pluripotent stem cells from mouse embryonic and adult fibroblast cultures by defined factors. Cell, 126 (4), 663-676.

(22) Takahashi, K., Tanabe, K., Ohnuki, M. et al. (2007) Induction of pluripotent stem cells from adult human fibroblasts by defined factors. Cell, 131 (5), 861-872.

(23) Maherali, N., Sridharan, R., Xie, W. et al. (2007) Directly reprogrammed fibroblasts show global epigenetic remodeling and widespread tissue contribution. Cell Stem Cell, 1 (1), 55-70.

(24) Mikkelsen, T.S., Hanna, J., Zhang, X. et al. (2008) Dissecting direct reprogramming through integrative genomic analysis. Nature, 454 (7200), 49-55.

(25) Okita, K., Ichisaka, T., and Yamanaka, S. (2007) Generation of germline-competent induced pluripotent stem cells. Nature, 448 (7151), 313-317.

(26) Wernig, M., Meissner, A., Foreman, R. et al. (2007) In vitro reprogramming of fibroblasts into a pluripotent ES-cell-like state. Nature, 448 (7151), 318-324.

(27) Yu, J., Vodyanik, M.A., Smugga-Otto, K. et al. (2007) Induced pluripotent stem cell lines derived from human somatic cells. Science, 318 (5858), 1917-1920.

(28) Lowry, W.E., Richter, L., Yachechko, R. et al. (2008) Generation of human induced pluripotent stem cells from dermal fibroblasts. Proceedings of the National Academy of Sciences of the United States of America, 105 (8), 2883-2888.

(29) Park, I.H., Zhao, R., West, J.A. et al. (2008) Reprogramming of human somatic cells to pluripotency with defined factors. Nature, 451 (7175), 141-146.

(30) Gonzalez, F., Boue, S., and Izpisua Belmonte, J.C. (2011) Methods for making induced pluripotent stem cells: reprogramming a la carte. Nature Reviews Genetics, 12 (4), 231-242. 
(31) Maherali, N. and Hochedlinger, K. (2008) Guidelines and techniques for the generation of induced pluripotent stem cells. Cell Stem Cell, 3 (6), 595-605.

(32) Aasen, T., Raya, A., Barrero, M.J. et al. (2008) Efficient and rapid generation of induced pluripotent stem cells from human keratinocytes. Nature Biotechnology, 26 (11), 1276-1284.

(33) Giorgetti, A., Montserrat, N., Aasen, T. et al. (2009) Generation of induced pluripotent stem cells from human cord blood using OCT4 and SOX2. Cell Stem Cell, 5 (4), 353-357.

(34) Loh, Y.H., Agarwal, S., Park, I.-H. et al. (2009) Generation of induced pluripotent stem cells from human blood. Blood, 113 (22), 5476-5479.

(35) Maekawa, M., Yamaguchi, K., Nakamura, T. et al. (2011) Direct reprogramming of somatic cells is promoted by maternal transcription factor Glis1. Nature, 474 (7350), 225-229.

(36) Han, J., Yuan, P., Yang, H. et al. (2010) Tbx3 improves the germ-line competency of induced pluripotent stem cells. Nature, 463 (7284), 1096-1100.

(37) Gao, Y., Chen, J., Li, K. et al. (2013) Replacement of Oct4 by Tet1 during iPSC induction reveals an important role of DNA methylation and hydroxymethylation in reprogramming. Cell Stem Cell, 12 (4), 453-469.

(38) Hockemeyer, D., Soldner, F., Cook, E.G. et al. (2008) A drug-inducible system for direct reprogramming of human somatic cells to pluripotency. Cell Stem Cell, 3 (3), 346-353.

(39) Nakagawa, M., Koyanagi, M., Tanabe, K. et al. (2008) Generation of induced pluripotent stem cells without Myc from mouse and human fibroblasts. Nature Biotechnology, 26 (1), 101-106.

(40) Stadtfeld, M., Nagaya, M., Utikal, J. et al. (2008) Induced pluripotent stem cells generated without viral integration. Science, 322 (5903), 945-949.

(41) Fusaki, N., Ban, H., Nishiyama, A. et al. (2009) Efficient induction of transgene-free human pluripotent stem cells using a vector based on Sendai virus, an RNA virus that does not integrate into the host genome. Proceedings of the Japan Academy. Series B, Physical and Biological Sciences, 85 (8), 348-362.

(42) Ban, H., Nishishita, N., Fusaki, N. et al. (2011) Efficient generation of transgene-free human induced pluripotent stem cells (iPSCs) by temperature-sensitive Sendai virus vectors. Proceedings of the National Academy of Sciences of the United States of America, 108 (34), 14234-14239.

(43) Okita, K., Nakagawa, M., Hyenjong, H. et al. (2008) Generation of mouse induced pluripotent stem cells without viral vectors. Science, 322 (5903), 949-953.

(44) Gonzalez, F., Barragan, M., Tiscornia, G. et al. (2009) Generation of mouse-induced pluripotent stem cells by transient expression of a single nonviral polycistronic vector. Proceedings of the National Academy of Sciences of the United States of America, 106 (22), 8918-8922.

(45) Yu, J., Hu, K., Smuga-Otto, K. et al. (2009) Human induced pluripotent stem cells free of vector and transgene sequences. Science, 324 (5928), 797-801.

(46) Jia, F., Wilson, K.D., Sun, N. et al. (2010) A nonviral minicircle vector for deriving human iPS cells. Nature Methods, 7 (3), 197-199.

(47) Woltjen, K., Michael, I.P., Mohseni, P. et al. (2009) piggyBac transposition reprograms fibroblasts to induced pluripotent stem cells. Nature, 458 (7239), 766-770.

(48) Yusa, K., Rad, R., Takeda, J., and Bradley, A. (2009) Generation of transgene-free induced pluripotent mouse stem cells by the piggyBac transposon. Nature Methods, 6 (5), 363-369.

(49) Kim, D., Kim, C., Moon, J. et al. (2009) Generation of human induced pluripotent stem cells by direct delivery of reprogramming proteins. Cell Stem Cell, 4 (6), 472-476.

(50) Zhou, H., Wu, S., Joo, J.-Y. et al. (2009) Generation of induced pluripotent stem cells using recombinant proteins. Cell Stem Cell, 4 (5), 381-384.

(51) Warren, L., Manos, P.D., Ahfeldt, T. et al. Highly efficient reprogramming to pluripotency and directed differentiation of human cells with synthetic modified mRNA. Cell Stem Cell, 7 (5), 618-630.

(52) Anokye-Danso, F., Trivedi, C.M., Juhr, D. et al. (2011) Highly efficient miRNA-mediated reprogramming of mouse and human somatic cells to pluripotency. Cell Stem Cell, 8 (4), 376-388.

(53) Miyoshi, N., Ishii, H., Nagano, H. et al. (2011) Reprogramming of mouse and human cells to pluripotency using mature microRNAs. Cell Stem Cell, 8 (6), 633-638. 
(54) Huangfu, D., Maehr, R., Guo, W. et al. (2008) Induction of pluripotent stem cells by defined factors is greatly improved by small-molecule compounds. Nature Biotechnology, 26 (7), 795-797.

(55) Shi, Y., Tae Do, J., Desponts, C. et al. (2008) A combined chemical and genetic approach for the generation of induced pluripotent stem cells. Cell Stem Cell, 2 (6), 525-528.

(56) Zhang, Y., Li, W., Laurent, T., and Ding, S. (2012) Small molecules, big roles - the chemical manipulation of stem cell fate and somatic cell reprogramming. Journal of Cell Science, 125 (Pt 23), 5609-5620.

(57) Polo, J.M., Anderssen, E., Walsh, R.M. et al. (2012) A molecular roadmap of reprogramming somatic cells into iPS cells. Cell, 151 (7), 1617-1632.

(58) Papp, B. and Plath, K. (2013) Epigenetics of reprogramming to induced pluripotency. Cell, $152(6), 1324-1343$.

(59) Onder, T.T., Kara, N., Cherry, A. et al. (2012) Chromatin-modifying enzymes as modulators of reprogramming. Nature, $\mathbf{4 8 3}$ (7391), 598-602.

(60) Zhu, S., Wei, W., and Ding, S. (2011) Chemical strategies for stem cell biology and regenerative medicine. Annual Review of Biomedical Engineering, 13, 73-90.

(61) Kishigami, S., Mizutani, E., Ohta, H. et al. (2006) Significant improvement of mouse cloning technique by treatment with trichostatin A after somatic nuclear transfer. Biochemical and Biophysical Research Communications, 340 (1), 183-189.

(62) Rybouchkin, A., Kato, Y., and Tsunoda, Y. (2006) Role of histone acetylation in reprogramming of somatic nuclei following nuclear transfer. Biology of Reproduction, 74 (6), 1083-1089.

(63) Yoshida, Y., Takahashi, K., Okita, K. et al. (2009) Hypoxia enhances the generation of induced pluripotent stem cells. Cell Stem Cell, 5 (3), 237-241.

(64) Huangfu, D., Osafune, K., Maehr, R. et al. (2008) Induction of pluripotent stem cells from primary human fibroblasts with only Oct4 and Sox2. Nature Biotechnology, 26 (11), 1269 1275.

(65) Mali, P., Chou, B.K., Yen, J. et al. (2010) Butyrate greatly enhances derivation of human induced pluripotent stem cells by promoting epigenetic remodeling and the expression of pluripotency-associated genes. Stem Cells, 28 (4), 713-720.

(66) Boland, M.J., Hazen, J.L., Nazor, K.L. et al. (2009) Adult mice generated from induced pluripotent stem cells. Nature, 461 (7260), 91-94.

(67) Feng, B., Ng, J.H., Heng, J.C., and Ng, H.H. (2009) Molecules that promote or enhance reprogramming of somatic cells to induced pluripotent stem cells. Cell Stem Cell, 4 (4), 301-312.

(68) Kubicek, S., O'Sullivan, R.J., August, E.M. et al. (2007) Reversal of H3K9me2 by a smallmolecule inhibitor for the G9a histone methyltransferase. Molecular Cell, 25 (3), 473-481.

(69) Shi, Y., Desponts, C., Do, J.T. et al. (2008) Induction of pluripotent stem cells from mouse embryonic fibroblasts by Oct 4 and Klf4 with small-molecule compounds. Cell Stem Cell, 3 (5), 568-574.

(70) Feldman, N., Gerson, A., Fang, J. et al. (2006) G9a-mediated irreversible epigenetic inactivation of Oct-3/4 during early embryogenesis. Nature Cell Biology, 8 (2), 188-194.

(71) Masui, S., Nakatake, Y., Toyooka, Y. et al. (2007) Pluripotency governed by Sox2 via regulation of Oct3/4 expression in mouse embryonic stem cells. Nature Cell Biology, 9 (6), 625-635.

(72) Ellis, P., Fagan, B.M., Magness, S.T. et al. (2004) SOX2, a persistent marker for multipotential neural stem cells derived from embryonic stem cells, the embryo or the adult. Developmental Neuroscience, 26 (2-4), 148-165.

(73) Kim, J.B., Zaehres, H., Wu, G. et al. (2008) Pluripotent stem cells induced from adult neural stem cells by reprogramming with two factors. Nature, 454 (7204), 646-650.

(74) Li, W., Zaehres, H., Wu, G. et al. (2009) Generation of human-induced pluripotent stem cells in the absence of exogenous Sox2. Stem Cells, 27 (12), 2992-3000.

(75) Li, Y., Zhou, H., Abujarour, R. et al. (2011) Generation of iPSCs from mouse fibroblasts with a single gene, Oct 4 , and small molecules. Cell Research, 21 (1), 196-204.

(76) Wang, T., Chen, K., Zeng, X. et al. (2011) The histone demethylases Jhdm1a/1b enhance somatic cell reprogramming in a vitamin-C-dependent manner. Cell Stem Cell, 9 (6), 575587. 
(77) Wang, J., Lu, F., Ren, Q. et al. (2011) Novel histone demethylase LSD1 inhibitors selectively target cancer cells with pluripotent stem cell properties. Cancer Research, 71 (23), 72387249.

(78) Brueckner, B., Garcia Boy, R., Siedlecki, P. et al. (2005) Epigenetic reactivation of tumor suppressor genes by a novel small-molecule inhibitor of human DNA methyltransferases. Cancer Research, 65 (14), 6305-6311.

(79) Lee, J., Xia, Y., Son, M.-Y. et al. (2012) A novel small molecule facilitates the reprogramming of human somatic cells into a pluripotent state and supports the maintenance of an undifferentiated state of human pluripotent stem cells. Angewandte Chemie International Edition in English, 51 (50), 12509-12513.

(80) Yuan, X., Li, W., and Ding, S. (2011) Small molecules in cellular reprogramming and differentiation. Progress in Drug Research, 67, 253-266.

(81) Ichida, J.K., Blanchard, J., Lam, K. et al. (2009) A small-molecule inhibitor of tgf-Beta signaling replaces sox2 in reprogramming by inducing nanog. Cell Stem Cell, 5 (5), 491-503.

(82) Maherali, N. and Hochedlinger, K. (2009) Tgfbeta signal inhibition cooperates in the induction of iPSCs and replaces Sox2 and cMyc. Current Biology, 19 (20), 1718-1723.

(83) Woltjen, K. and Stanford, W.L. (2009) Preview. Inhibition of Tgf-beta signaling improves mouse fibroblast reprogramming. Cell Stem Cell, 5 (5), 457-458.

(84) Ying, Q.L., Nichols, J., Chambers, I., and Smith, A. (2003) BMP induction of Id proteins suppresses differentiation and sustains embryonic stem cell self-renewal in collaboration with STAT3. Cell, 115 (3), 281-292.

(85) Li, W., Wei, W., Zhu, S. et al. (2009) Generation of rat and human induced pluripotent stem cells by combining genetic reprogramming and chemical inhibitors. Cell Stem Cell, 4 (1), $16-19$.

(86) Lin, T., Ambasudhan, R., Yuan, X. et al. (2009) A chemical platform for improved induction of human iPSCs. Nature Methods, 6 (11), 805-808.

(87) Yuan, X., Wan, H., Zhao, X. et al. (2011) Brief report: combined chemical treatment enables Oct4-induced reprogramming from mouse embryonic fibroblasts. Stem Cells, 29 (3), 549-553.

(88) Li, R., Liang, J., Ni, S. et al. (2010) A mesenchymal-to-epithelial transition initiates and is required for the nuclear reprogramming of mouse fibroblasts. Cell Stem Cell, 7 (1), 51-63.

(89) Samavarchi-Tehrani, P., Golipour, A., David, L. et al. (2010) Functional genomics reveals a BMP-driven mesenchymal-to-epithelial transition in the initiation of somatic cell reprogramming. Cell Stem Cell, 7 (1), 64-77.

(90) Davidson, K.C., Adams, A.M., Goodson, J.M. et al. (2012) Wnt/ $\beta$-catenin signaling promotes differentiation, not self-renewal, of human embryonic stem cells and is repressed by Oct4. Proceedings of the National Academy of Sciences of the United States of America, 109 (12), 4485-4490.

(91) Merrill, B.J. (2012) Wnt pathway regulation of embryonic stem cell self-renewal. Cold Spring Harbor Perspectives in Biology, 4 (9), a007971.

(92) Sato, N., Meijer, L., Skaltsounis, L. et al. (2004) Maintenance of pluripotency in human and mouse embryonic stem cells through activation of Wnt signaling by a pharmacological GSK-3-specific inhibitor. Nature Medicine, 10 (1), 55-63.

(93) Grigoryan, T., Wend, P., Klaus, A., and Birchmeier, W. (2008) Deciphering the function of canonical Wnt signals in development and disease: conditional loss- and gain-of-function mutations of beta-catenin in mice. Genes \& Development, 22 (17), 2308-2341.

(94) Marson, A., Foreman, R., Chevalier, B. et al. (2008) Wnt signaling promotes reprogramming of somatic cells to pluripotency. Cell Stem Cell, 3 (2), 132-135.

(95) Lluis, F., Pedone, E., Pepe, S., and Cosma, M.P. (2008) Periodic activation of Wnt/beta-catenin signaling enhances somatic cell reprogramming mediated by cell fusion. Cell Stem Cell, 3 (5), 493-507.

(96) Lluis, F., Ombrato, L., Pedone, E. et al. (2011) T-cell factor 3 (Tcf3) deletion increases somatic cell reprogramming by inducing epigenome modifications. Proceedings of the National Academy of Sciences of the United States of America, 108 (29), 11912-11917.

(97) Silva, J., Barrandon, O., Nichols, J. et al. (2008) Promotion of reprogramming to ground state pluripotency by signal inhibition. PLoS Biol., 6 (10), e253. 
(98) Lyssiotis, C.A., Foreman, R.K., Staerk, J. et al. (2009) Reprogramming of murine fibroblasts to induced pluripotent stem cells with chemical complementation of Klf4. Proceedings of the National Academy of Sciences of the United States of America, 106 (22), 8912-8917.

(99) Watanabe, K., Ueno, M., Kamiya, D. et al. (2007) A ROCK inhibitor permits survival of dissociated human embryonic stem cells. Nature Biotechnology, 25 (6), 681-686.

(100) Chen, T., Yuan, D., Wei, B. et al. (2010) E-cadherin-mediated cell-cell contact is critical for induced pluripotent stem cell generation. Stem Cells, 28 (8), 1315-1325.

(101) Ohgushi, M., Matsumura, M., Eiraku, M. et al. (2010) Molecular pathway and cell state responsible for dissociation-induced apoptosis in human pluripotent stem cells. Cell Stem Cell, 7 (2), 225-239.

(102) Li, Z. and Rana, T.M. (2012) A kinase inhibitor screen identifies small-molecule enhancers of reprogramming and iPS cell generation. Nature Communications, 3, 1085.

(103) Lee, D.F., Su, J., Ang, Y.S. et al. (2012) Regulation of embryonic and induced pluripotency by aurora kinase-p53 signaling. Cell Stem Cell, 11 (2), 179-194.

(104) Staerk, J., Lyssiotis, C.A., Medeiro, L.A. et al. (2011) Pan-Src family kinase inhibitors replace Sox 2 during the direct reprogramming of somatic cells. Angewandte Chemie International Edition in English, 50 (25), 5734-5736.

(105) Wang, Y. and Adjaye, J. (2011) A cyclic AMP analog, 8-Br-cAMP, enhances the induction of pluripotency in human fibroblast cells. Stem Cell Reviews, 7 (2), 331-341.

(106) Zhu, S., Li, W., Zhou, H. et al. (2010) Reprogramming of human primary somatic cells by OCT4 and chemical compounds. Cell Stem Cell, 7 (6), 651-655.

(107) Collado, M., Blasco, M.A., and Serrano, M. (2007) Cellular senescence in cancer and aging. Cell, 130 (2), 223-233.

(108) Utikal, J., Polo, J.M., Stadtfeld, M. et al. (2009) Immortalization eliminates a roadblock during cellular reprogramming into iPS cells. Nature, 460 (7259), 1145-1148.

(109) Banito, A., Rashid, S.T., Acosta, J.C. et al. (2009) Senescence impairs successful reprogramming to pluripotent stem cells. Genes \& Development, 23 (18), 2134-2139.

(110) Esteban, M.A., Wang, T., Qin, B. et al. (2010) Vitamin C enhances the generation of mouse and human induced pluripotent stem cells. Cell Stem Cell, 6 (1), 71-79.

(111) González, F., Georgieva, D., Vanoli, F. et al. (2013) Homologous recombination DNA repair genes play a critical role in reprogramming to a pluripotent state. Cell Reports, 3 (3), 651-660.

(112) Hong, H., Takahashi, K., Ichisaka, T. et al. (2009) Suppression of induced pluripotent stem cell generation by the p53-p21 pathway. Nature, 460 (7259), 1132-1135.

(113) Kawamura, T., Suzuki, J., Wang, Y.V. et al. (2009) Linking the p53 tumour suppressor pathway to somatic cell reprogramming. Nature, $\mathbf{4 6 0}$ (7259), 1140-1144.

(114) Marion, R.M., Strati, K., Li, H. et al. (2009) A p53-mediated DNA damage response limits reprogramming to ensure iPS cell genomic integrity. Nature, 460 (7259), 1149-1153.

(115) Zhao, Y., Yin, X., Qin, H. et al. (2008) Two supporting factors greatly improve the efficiency of human iPSC generation. Cell Stem Cell, 3 (5), 475-479.

(116) Li, H., Collado, M., Villasante, A. et al. (2009) The Ink4/Arf locus is a barrier for iPS cell reprogramming. Nature, $\mathbf{4 6 0}$ (7259), 1136-1139.

(117) Okita, K., Matsumura, Y., Sato, Y. et al. (2011) A more efficient method to generate integrationfree human iPS cells. Nature Methods, 8 (5), 409-412.

(118) Vafa, O., Wade, M., Kern, S. et al. (2002) c-Myc can induce DNA damage, increase reactive oxygen species, and mitigate p53 function: a mechanism for oncogene-induced genetic instability. Molecular Cell, 9 (5), 1031-1044.

(119) von Zglinicki, T. (2002) Oxidative stress shortens telomeres. Trends in Biochemical Sciences, 27 (7), 339-344.

(120) Chen, T., Shen, L., Yu, J. et al. (2011) Rapamycin and other longevity-promoting compounds enhance the generation of mouse induced pluripotent stem cells. Aging Cell, 10 (5), 908-911.

(121) Chung, T.L., Brena, R.M., Kolle, G. et al. (2010) Vitamin C promotes widespread yet specific DNA demethylation of the epigenome in human embryonic stem cells. Stem Cells, 28 (10), $1848-1855$. 
(122) Stadtfeld, M., Apostolou, E., Ferrari, F. et al. (2012) Ascorbic acid prevents loss of Dlk1-Dio3 imprinting and facilitates generation of all-iPS cell mice from terminally differentiated B cells. Nature Genetics, 44(4), 398-405, S1-2.

(123) Davis, R.L., Weintraub, H., and Lassar, A.B. (1987) Expression of a single transfected cDNA converts fibroblasts to myoblasts. Cell, 51 (6), 987-1000.

(124) Vierbuchen, T., Ostermeier, A., Pang, Z.P. et al. (2010) Direct conversion of fibroblasts to functional neurons by defined factors. Nature, 463 (7284), 1035-1041.

(125) Ieda, M., Fu, J.D., Delgado-Olquin, P. et al. (2010) Direct reprogramming of fibroblasts into functional cardiomyocytes by defined factors. Cell, 142 (3), 375-386.

(126) Zhou, Q., Brown, J., Kanarek, A. et al. (2008) In vivo reprogramming of adult pancreatic exocrine cells to beta-cells. Nature, 455 (7213), 627-632.

(127) Efe, J.A., Hilcove, S., Kim, J. et al. (2011) Conversion of mouse fibroblasts into cardiomyocytes using a direct reprogramming strategy. Nature Cell Biology, 13 (3), 215-222.

(128) Kim, J., Efe, J.A., Zhu, S. et al. (2011) Direct reprogramming of mouse fibroblasts to neural progenitors. Proceedings of the National Academy of Sciences of the United States of America, 108 (19), 7838-7843.

(129) Ring, K.L., Tong, L.M., Balestra, M.E. et al. (2012) Direct reprogramming of mouse and human fibroblasts into multipotent neural stem cells with a single factor. Cell Stem Cell, 11 (1), 100-109.

(130) Szabo, E., Rampalli, S., Risueño, R.M. et al. (2010) Direct conversion of human fibroblasts to multilineage blood progenitors. Nature, 468 (7323), 521-526.

(131) Ma, T., Xie, M., Laurent, T. et al. (2013) Progress in the reprogramming of somatic cells. Circulation Research, 112 (3), 562-574.

(132) Ladewig, J., Mertens, J., Kesavan, J. et al. (2012) Small molecules enable highly efficient neuronal conversion of human fibroblasts. Nature Methods, 9 (6), 575-578.

(133) Darabi, R., Arpke, R.W., Irion, S. et al. (2012) Human ES- and iPS-derived myogenic progenitors restore DYSTROPHIN and improve contractility upon transplantation in dystrophic mice. Cell Stem Cell, 10 (5), 610-619.

(134) Ahfeldt, T., Schinzel, R.T., Lee, Y.-K. et al. (2012) Programming human pluripotent stem cells into white and brown adipocytes. Nature Cell Biology, 14 (2), 209-219.

(135) Pang, Z.P., Yang, N., Vierbuchen, T. et al. (2011) Induction of human neuronal cells by defined transcription factors. Nature, 476 (7359), 220-223.

(136) Hester, M.E., Murtha, M.J., Song, S.W. et al. (2011) Rapid and efficient generation of functional motor neurons from human pluripotent stem cells using gene delivered transcription factor codes. Molecular Therapy, 19 (10), 1905-1912.

(137) Li, W., Tian, E., Chen, Z.-X. et al. (2012) Identification of Oct4-activating compounds that enhance reprogramming efficiency. Proceedings of the National Academy of Sciences of the United States of America, 109 (51), 20853-20858.

(138) Gore, A., Li, Z., Fung, H.L. et al. (2011) Somatic coding mutations in human induced pluripotent stem cells. Nature, 471 (7336), 63-67.

(139) Hussein, S.M., Batada, N.N., Vuoristo, S. et al. (2011) Copy number variation and selection during reprogramming to pluripotency. Nature, 471 (7336), 58-62.

(140) Laurent, L.C., Ulitsky, I., Slavin, I. et al. (2011) Dynamic changes in the copy number of pluripotency and cell proliferation genes in human ESCs and iPSCs during reprogramming and time in culture. Cell Stem Cell, 8 (1), 106-118.

(141) Mayshar, Y., Ben-David, U., Lavon, N. et al. (2010) Identification and classification of chromosomal aberrations in human induced pluripotent stem cells. Cell Stem Cell, 7 (4), 521-531.

(142) Lee, Y.L., Peng, Q., Fong, S.W. et al. (2012) Sirtuin 1 facilitates generation of induced pluripotent stem cells from mouse embryonic fibroblasts through the miR-34a and p53 pathways. PLoS One, 7 (9), e45633. 


\title{
10
}

\section{Chemicals Facilitating Reprogramming: Targeting the SAM Binding Site to Identify Novel Methyltransferase Inhibitors}

\author{
Jeong-Do Kim ${ }^{1}$, Jong S. Rim ${ }^{1}$, Robert B. Crochet ${ }^{2}$, Yong-Hwan Lee ${ }^{2}$, \\ Jaroslaw Staszkiewicz, Ru Gao ${ }^{1}$, and Kenneth J. Eilertsen ${ }^{1,3}$ \\ ${ }^{I}$ NuPotential, Inc., USA \\ ${ }^{2}$ Department of Biological Science, Louisiana State University, USA \\ ${ }^{3}$ Pennington Biomedical Research Center, LSU System, USA
}

\subsection{Introduction}

A variety of reprogramming strategies are being developed that address the safety concerns associated with protocols dependent on genetic alterations such as virus-based reprogramming. Approaches include the use of nonintegrating plasmids and mRNA [1-6], microRNA [7], excisable transposons [8,9], and proteins $[10,11]$. An alternative method to enhance reprogramming is the use of a small-molecule inhibitor, or a cocktail of small-molecule inhibitors. Several chemicals have been reported to either improve reprogramming efficiency or substitute for specific factors. DNA and histone methyltransferase inhibitors, in particular, have shown tremendous promise in improving reprogramming efficiencies and generating safer induced pluripotent stem cells (iPSCs). These enzymes play important roles in controlling gene expression and are gaining attention in drug discovery and medicinal chemistry due to their association with a variety of pathologies. The objective

Chemical Biology in Regenerative Medicine: Bridging Stem Cells and Future Therapies, First Edition. Edited by Charles C. Hong, Ada S. Ao, and Jijun Hao.

(C) 2014 John Wiley \& Sons, Ltd. Published 2014 by John Wiley \& Sons, Ltd. 
of our studies is to identify novel lead compounds for human DNMT1 and histone methyltransferase inhibition targeting the S-adenosylmethionine (SAM) binding pocket of these enzymes, utilizing computer-based approaches.

\subsection{DNA Methyltransferases, Inhibition, and Reprogramming}

Reprogramming of mouse and human fibroblasts is an inefficient process. For mouse embryonic fibroblasts (MEFs), Mikkelson and colleagues reported that only a small percentage of cells transduced with defined transcription factors reactivated endogenous Nanog. Moreover, the majority of infected cells appeared to be trapped in a partially reprogrammed state [12-14]. They further demonstrated that partially reprogrammed cells could be induced to become fully reprogrammed by treatment with the DNA methyltransferase inhibitor 5-aza-cytidine (5-AZA). In addition, treatment with 5-AZA improved embryonic stem cell (ESC)-like colony formation by fourfold. We have proposed that epigenetic modification increases somatic cell reprogramming efficiency through (i) direct activation of pluripotent gene expression and (ii) activation of a pluripotent transcription factor network that is unique in pluripotent stem cells, including ESCs [15]. To test this hypothesis, we used a combination of two epigenetic modifying agents: a DNMT inhibitor and a histone-modifying enzyme inhibitor [15]. Primary cultures from three different origins of human dermal fibroblast (adult, neonatal, and fetal) were treated with epigenetic modifying agents with and without ectopic expression of Oct 4 and Nanog genes. As shown in Figure 10.1, inhibition of DNMT in human dermal fibroblast cultures induced the expression of Oct4, Nanog, and Sox 2 through auto- (Figure 10.1a) and reciprocal activation of the endogenous pluripotent transcription factor network (Figure 10.1b). In addition, DNMT inhibitor treatment, both before and after lentiviral-induced pluripotent factor expression, increased the efficiency of colony maturation by measuring co-expression of Sox 2 (an early pluripotent marker) and TRA1-60 (a late pluripotent marker) from alkaline phosphatase (AP)-positive colonies (Figure 10.2). These results indicate that DNA methylation is an important epigenetic barrier that can limit the production of fully reprogrammed cells and that reprogramming efficiency can be improved by inhibiting specific repressive epigenetic regulatory components at the level of ESC-like colony formation and maturation. DNA methylation in mammalian cells occurs through the transfer of a methyl group from the universal cofactor SAM to cytosine of CpG dinucleotides $[16,17]$ and is catalyzed by two types of DNA methyltransferase: a maintenance methyltransferase, DNMT1, and de novo methyltransferases, DNMT3a and 3b $[18,19]$.

\subsection{DNMT Inhibitors}

DNMT inhibitors are categorized into two groups: (i) nucleoside analogs, which incorporate into DNA and/or RNA and inhibit DNA methylation by trapping DNMTs, and (ii) nonnucleoside analogs, which directly inhibit DNMT activity by blocking the SAM pocket of the enzyme [20,21]. Only two DNMT inhibitors derived from the nucleoside cytidine, 5-azacytidine, and 5-aza-2'-deoxycytidine (decitabine) are currently in clinical trials for anticancer therapy $[22,23]$. However, despite their use in the successful treatment of 

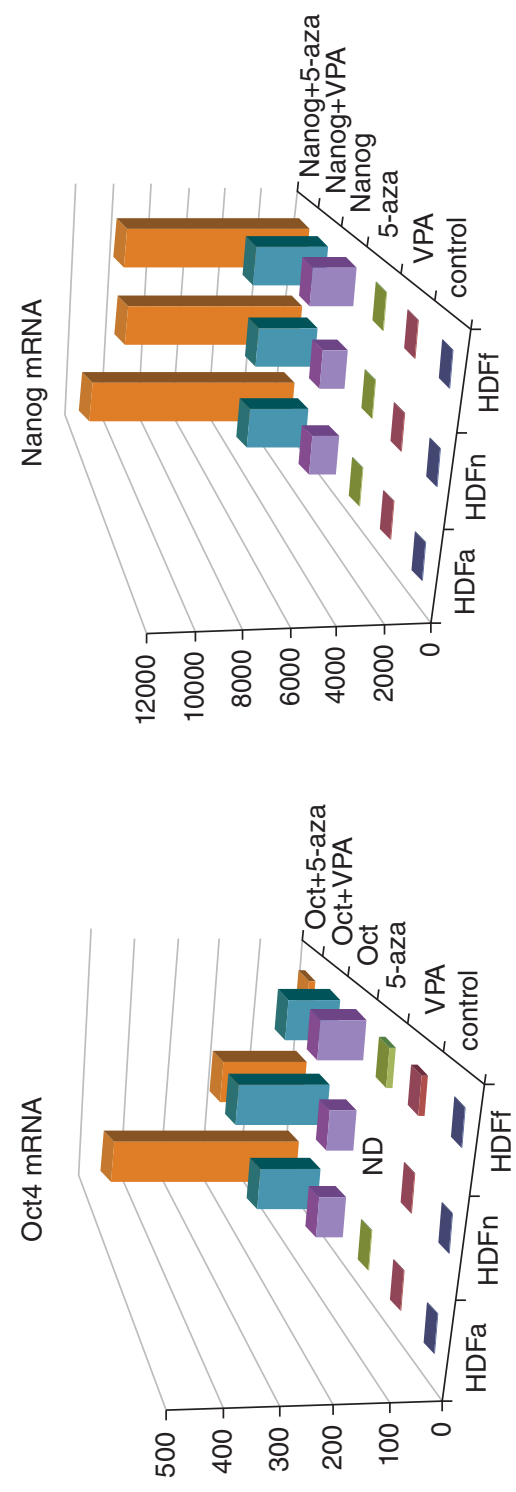

త్ర

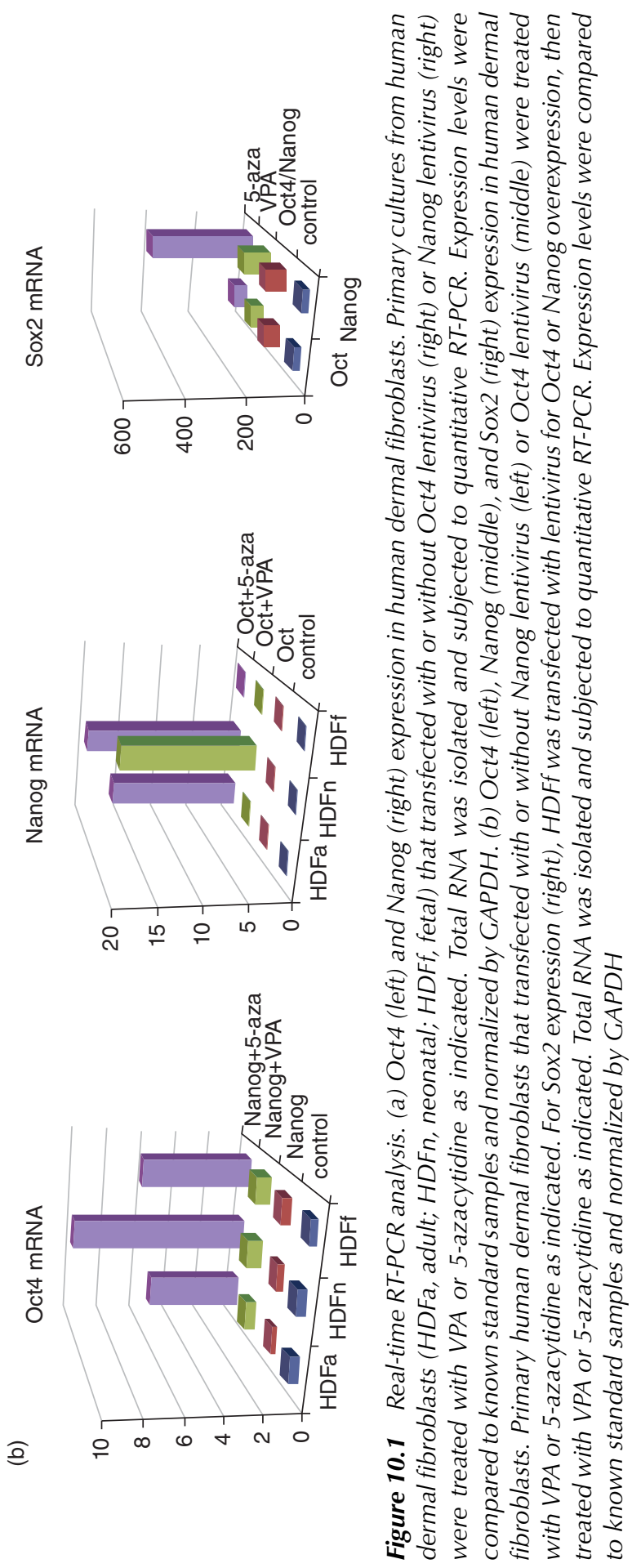



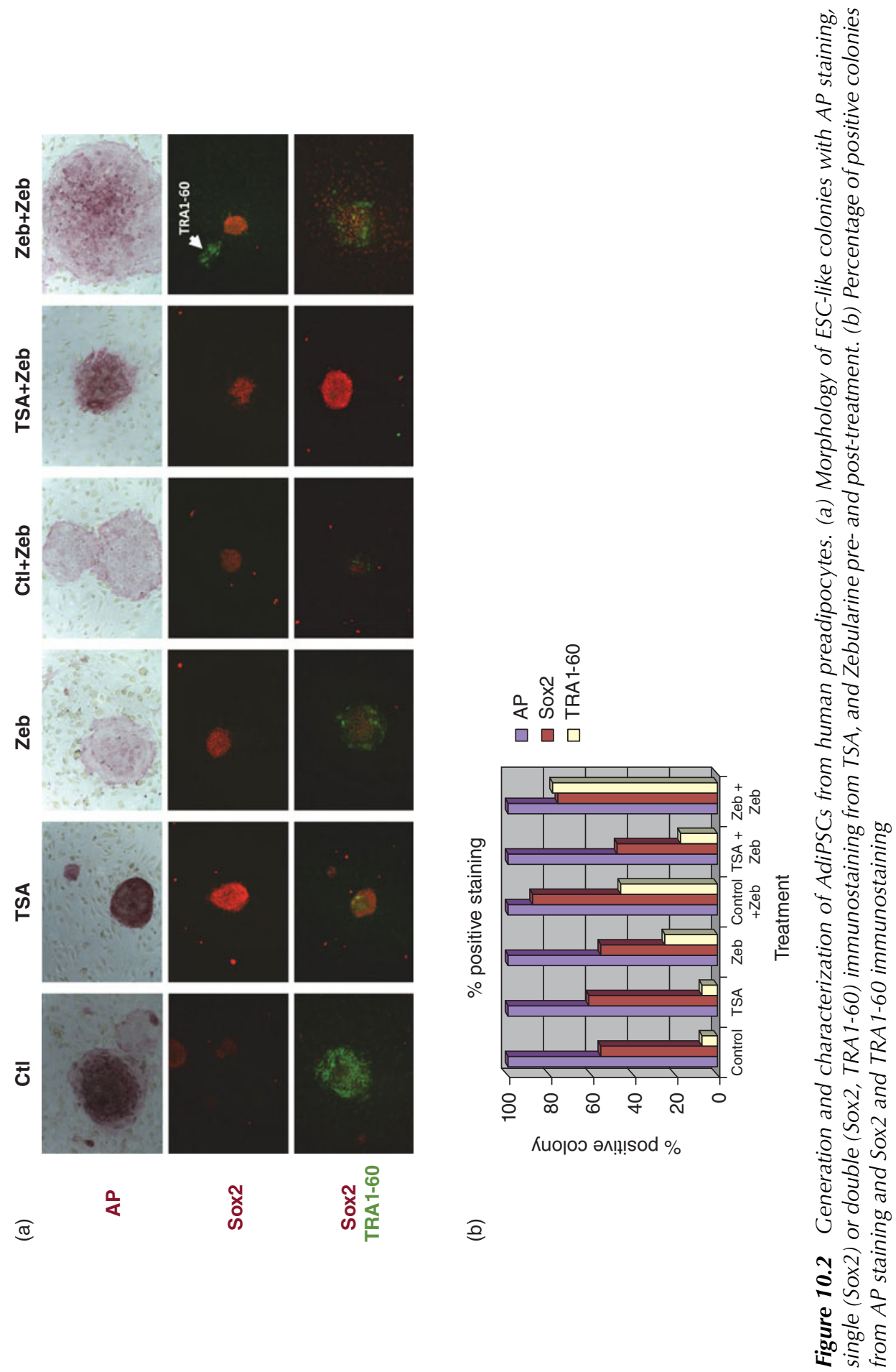
cancers such as acute lymphoblastic leukemia and myelodysplastic syndrome, these drugs have shown severe cytotoxicity in patients due to random incorporation into all deoxycytidine and cytidine sites of DNA and/or RNA, followed by DNMT depletion [24-26]. Most DNMT inhibitors were identified by serendipity. In contrast, RG108 was the first DNMT inhibitor identified and developed by virtual screening using 3D homology modeling of the catalytic domain of DNMT1. RG108 has shown comparatively low cytotoxicity in human cell lines, but the inhibition rate of RG108 for the purified DNMT1 is low $(<20 \%)$ compared to that of S-adenosylhomocysteine (SAH) (> 70\%), a demethylated form of SAM $[27,28]$. There is a need to develop next-generation DNMT inhibitors for therapeutic and reprogramming applications. Our efforts to develop a new class of DNMT inhibitor are centered on the use of computer-based approaches with an experimental crystal structure (3SWR).

\subsection{Histone Methyltransferases, Inhibition, and Reprogramming}

Protein lysine methylation is a key signaling mechanism in eukaryotic cells. Protein lysine methyltransferases (PKMTs) catalyze the transfer of a methyl group from SAM to the $\varepsilon$-amino group of lysine residues of proteins, including histones [29]. PKMTs show substantial variations in protein substrate selectivity and the degree of methylation on lysine from mono- to di- to trimethylation. At present few small-molecule probes exist to dissect the functionality of PKMTs.

Histone methyltransferases can be subdivided into three classes: the SET domaincontaining lysine methyltransferases and the non-SET-domain lysine methyltransferases and arginine methyltransferases (PRMTs). The focus of this chapter is on histone lysine methyltransferases. All of them use SAM as a cosubstrate for the methylation reaction and generate $\mathrm{SAH}$ as a byproduct. Histone methylation does not alter the charge of the lysine residue in question. However, it does affect the basicity, hydrophobicity, and size of the amino acid side-chain group, which affects the affinity of proteins that recognize such side chains [30]. Although these changes are subtle, there are proteins that are able to bind selectively to certain methylated residues based on specific structural motifs [31] (for review see [32]). Chromodomains recognize mono-, di-, or trimethylated lysine residues; Tudor domains bind trimethylated lysines; plant homeo domain (PHD) fingers bind di- or trimethylated lysines; malignant brain tumor (MBT) domains bind mono- and dimethylated lysines.

Histone lysine methylation is associated with both transcriptional activation and repression. For example, methylation of the tumor-suppressor protein p53 on K370 by Smyd2 [33] or on K382 by SET8 [34] leads to inhibition of transcriptional activity, whereas methylation on K372 by Set9 results in transcriptional activation [35]. The lysine methyltransferase G9a has recently been shown to dimethylate p53 on K373. This was correlated with inactivity of p53 [36]. Its impact on apoptotic processes and its overexpression in various cancer types suggest that $\mathrm{G} 9 \mathrm{a}$ is a putative oncogene.

G9a is a Histone3 Lysine9 (H3K9) methyltransferase and has been reported to be overexpressed in a variety of cancers [36-38]. G9a has also been shown to be involved in cocaine addiction [39], mental retardation [40], and DNA methylation in mouse ESCs. GLP is a closely related protein which shares $80 \%$ sequence identity with G9a and forms a 
heterodimer with it. Both G9a and GLP catalyze mono- and dimethylation of H3K9. Inhibition of G9a and GLP using small-molecule inhibitors has been demonstrated to facilitate the reprogramming of mouse neural precursor cells (NPCs) into iPSCs. Specfically, the G9a methyltransferase inhibitor BIX-01294 (BIX) was shown to improve the reprogramming efficiencies of OK (Oct4, Klf4)-infected NPCs by nearly eightfold [41]. G9a has been reported to mediate the repression of Oct4 [42] and Nanog [43]. Thus, it is possible that G9a inhibition could facilitate the reactivation of silenced Oct4 and Nanog and promote reprogramming.

The beneficial effect of BIX on reprogramming was further substantiated using MEFs [44]. BIX treatment in combination with the DNA methyltransferase inhibitor RG108 and L-calcium channel agonist BayK8644 (BayK) signficantly enhanced the reprogramming of BIX-treated MEFs transduced with Oct4 and Klf4. Thus, the combination of epigenetic inhibitors with other target inhibitors may be required to overcome resistance to reprogramming and induce responsiveness. The development of selective, potent, and cell-active chemical inhibitors would be extremely valuable in furthering the efficiency and elucidating the mechanisms of somatic cell reprogramming.

In addition, suppression of the histone methyltransferases DOT1L and SUV39H1 improved reprogramming. In particular, inhibition of the H3K79 histone methyltransferase DOT1L by shRNA or a small-molecule inhibitor increased the number of iPSC colonies, while substituting for KLF4 and MYC [45]. Onder et al. further reported that fibroblastspecific genes associated with the epithelial-mesenchymal transition lost H3K79me 2 and that DOT1L inhibition facilitated the loss of the mark from genes typically repressed in the pluripotent state. These studies further suggest that specific chromatin-modifying enzymes can be modulated to improve the efficiency of reprogramming and that the production of iPSCs using only small molecules may be possible.

\subsection{Inhibitors of Lysine Methyltransferases}

Methyltransferases can be inhibited by analogs of the methyl group donor SAM. There are three known cosubstrate analogs that inhibit a variety of methyltransferases: (i) sinefungin, an antibiotic compound that is structurally similar to SAM; (ii) SAH, the demethylated cosubstrate of SAM, which serves as a feedback inhibitor; and (iii) methylthioadenosine. All of these inhibitors lack selectivity [46]. There are only a few known drug-like inhibitors of lysine methyltransferases, most of which were discovered by random screening approaches. The first inhibitor was chaetocin, a fungal mycotoxin. It inhibits both the Drosophila melanogaster enzyme Su(var)3-9 with an IC50 of $0.8 \mathrm{mM}$ and G9a with an IC50 of $2.5 \mathrm{mM}$ [47]. The compound BIX-01294 was identified in a combined virtual and high-throughput screening approach and shown to be an inhibitor of G9a with an IC50 of $3 \mathrm{mM}$. Cultured cells treated with BIX-01294 showed a reduction of histone H3K9 dimethylation and a decrease in cell number. In the same screening procedure, the compound BIX-01338 was discovered as a nonselective inhibitor of methyltransferases. It did not show any selectivity between lysine and arginine methyltransferases with an IC50 of $5 \mathrm{mM}$ for G9a [48].

Recently, UNC0224 has been identified as a new inhibitor for G9a with an IC50 of $15 \mathrm{nM}$. UNC0224 (2,4-diamino-7-aminoalkoxyquinazoline) was developed by modifying BIX-01294 in a structure-guided manner. It demonstrated a 1000-fold increase in selectivity 
for G9a compared to SET7/9 and Set8. The crystal structure of G9a in complex with BIX01294 reveals the interactions of the inhibitor with the protein at the molecular level [49]. BIX-01294 forms two hydrogen bonds to Asp1131 and Asp1140 at the entrance of the substrate-binding pocket and two methoxy groups show van der Waals interactions with the deeper part of the pocket. Interestingly, the inhibitor is not involved in direct interaction with the residues of the catalytic site or the cofactor SAM. Based on such structural reasoning, the dimethylaminopropoxy side chain in UNC0224 was attached in order to obtain binding to the lysine tunnel, thereby increasing inhibitory activity.

\subsection{Identification of DNMT1 Inhibitor Candidates Using Virtual Screening}

In order to identify novel compounds with a potential DNMT1 inhibition activity, a small molecule library of $\sim 18$ million $2 \mathrm{D}$ structures was downloaded from ZINC (http://zinc.docking.org). Subsequently, an approximately 1.6 million compound library was prepared by clustering the 18 million compounds into 1.6 million groups and then enumerating conformers, tautomers, protomers, and stereoisomers. Ligand-based screenings were performed using SAM as a template for a 2D ligand similarity search and the 3D coordinates for SFG, taken from the DNMT1 complex structure, for a 3D similarity search. Using these two compound libraries, the top 256 and 17000 compounds were selected from 2D and 3D searches, respectively, based on the Tanimoto scores, which represent the similarity between the query compound and the target compound. Next, structure-based docking was carried out on the selected compounds using the widely distributed molecular docking software Vina, an improved version of AutoDock, which is an open-source program [50]. The human DNMT1 in complex with SFG (3SWR) were extracted from the Protein Data Bank (RCSB PDB) and a grid map was generated around the SAM site to cover the entire catalytic pocket using AutoDockTools. After docking, the top-scoring binding pose from each of the top-scoring compounds was visually evaluated using PyMOL [51]. Figure 10.3 shows the surface map of human DNMT1 with SFG (a), an entire SAM binding pocket (b), an original SFG binding mode (c), and the predicted binding modes for four of the top 46 "hit" compounds. From visualization of the docking results, two distinct binding modes were predicted. Of the 46 compounds, $30 \%$ favored the standard substrate binding mode (d-f) mimicking SFG (c). Meanwhile, $70 \%$ favored a new, alternative binding mode $(\mathrm{g}-\mathrm{i})$ in which the hydrophobic groups of inhibitors occupy the presumed site for cytosine from $\mathrm{CpG}$, the methyl group acceptor. Analysis of the two binding modes revealed that the catalytic pocket contains one hydrophilic region and two hydrophobic regions (d, g), of which SAM, SAH, and sinefungin occupy only two, suggesting that compounds able to cover all three binding regions may demonstrate increased target-selectivity for DNMT1.

\subsubsection{Functional Screening Using a DNMT1 Activity Assay}

The top 46 potential inhibitor candidates identified by docking were obtained and tested for DNMT1 inhibition activity using a DNMT1 ELISA (enzyme-linked immunosorbent assay BPS Bioscience) (Figure 10.4a) at concentrations of 10 and $100 \mu \mathrm{M}$ for each compound. 
(a)

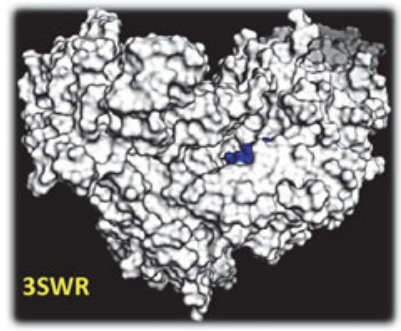

(d)

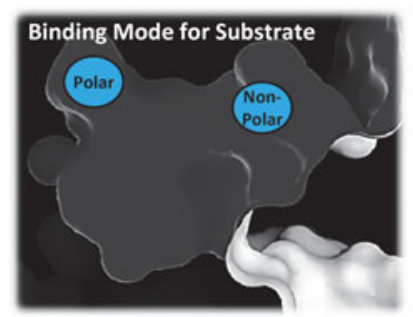

(g)

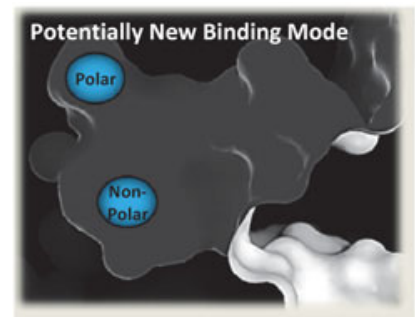

(b)

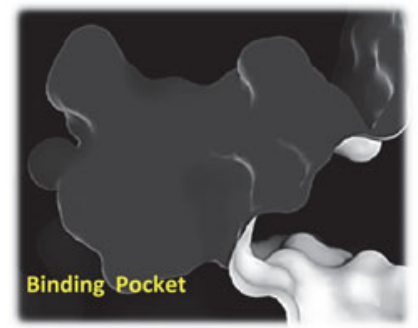

(e)

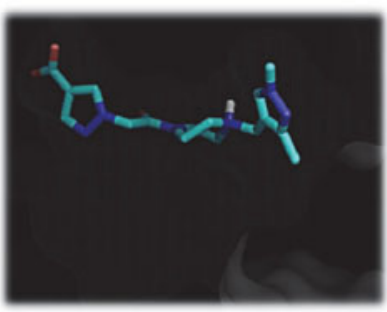

(h)

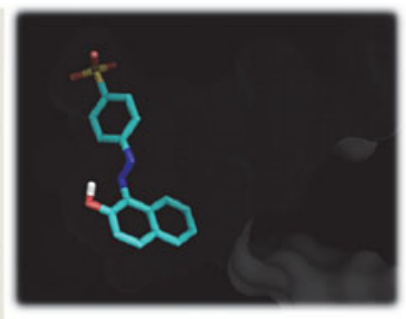

(c)

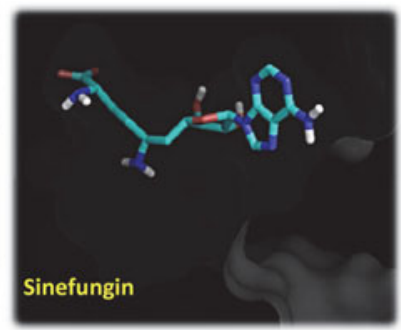

(f)

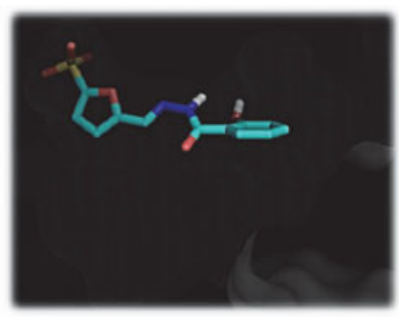

(i)

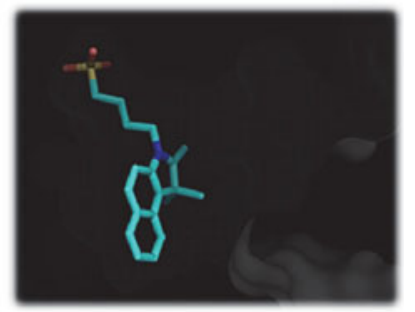

Figure 10.3 Binding-mode predictions of virtual docking with human DNMT1. (a) Diagram of DNMT1 in complex with SFG. SFG is occupying the SAM site of DNMT1, which is the target site for our screening. (b) SAM binding pocket. (c) An SFG binding pose in complex structure. $(d-i)$ Two types of predicted binding mode from docking and examples

The immobilized duplex DNAs containing several $\mathrm{CpG}$ sites were methylated by purified DNMT1 in the presence of each test compound $(10$ and $100 \mu \mathrm{M})$. Methylated cytosines were recognized by an anti-5-methylcytosine antibody. After adding HRP (horseradish peroxidase)-labeled secondary antibody, the plate was measured by luminometer, which detects chemiluminescence produced by HRP substrate reaction. The relative activity, as a percentage for each compound, was determined via comparison with the full activity of the enzyme. RG108 and SAH were used as reference compounds. Eight out of forty-six compounds showed higher DNMT1 inhibition rates than RG108, a known nonnucleoside DNMT inhibitor (Figure 10.4c). Surprisingly, one of them, NuP_0148, showed $\sim 77$ and $\sim 90 \%$ inhibition rates at concentrations of 10 and $100 \mu \mathrm{M}$, respectively. This finding validates our computer-based screening approach for identification of new lead molecules for DNMT1 inhibition. 


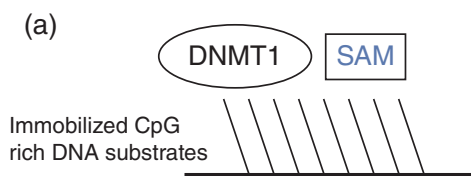

96 well plate

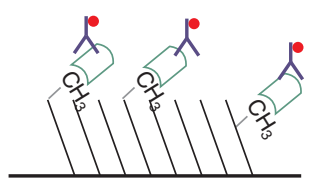

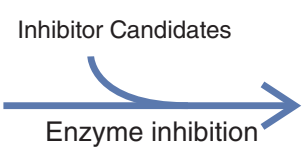
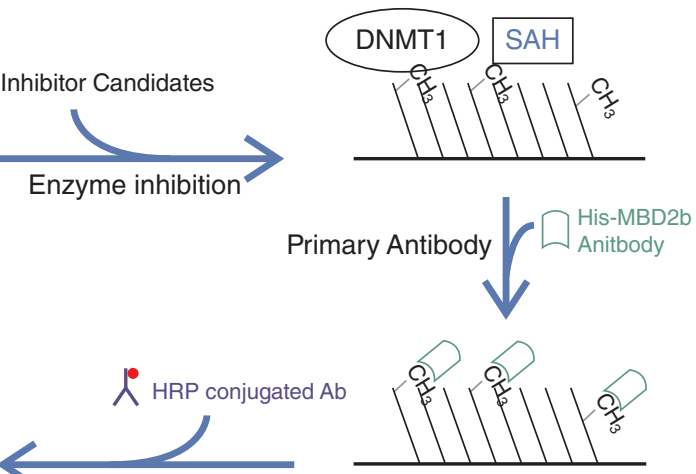

Secondary Antibody (b)

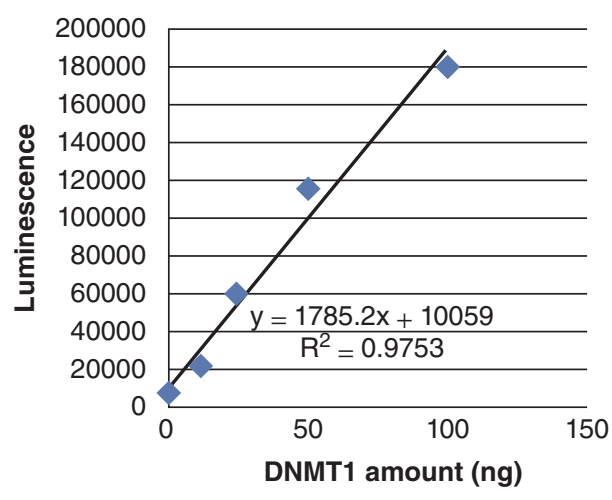

(c)

\begin{tabular}{|c|c|c|}
\hline ID & $10 u M$ & $100 u M$ \\
\hline NSC363232 & $23.1 \%$ & $10.2 \%$ \\
\hline NSC114103 & $87.9 \%$ & $68.9 \%$ \\
\hline ZINC47034061 & $84.4 \%$ & $83.6 \%$ \\
\hline ZINC40108600 & $94.3 \%$ & $80.6 \%$ \\
\hline ZINC04702530 & $83.4 \%$ & $85.3 \%$ \\
\hline ZINC10163939 & $90.5 \%$ & $72.0 \%$ \\
\hline ZINC25170704 & $90.4 \%$ & $79.2 \%$ \\
\hline ZINC03412030 & $87.9 \%$ & $75.6 \%$ \\
\hline RG108 & $87.5 \%$ & $85.8 \%$ \\
\hline SAH & $12.2 \%$ & $4.7 \%$ \\
\hline
\end{tabular}

Figure 10.4 Functional screening of DNMT1 inhibitor candidates. Scheme (a) and standard curve (b) of DNMT1 ELISA. (c) Relative DNMT1 activity of the inhibitor candidates

\subsection{Targeting the SAM Binding Site to Identify Novel HMT Inhibitors}

Prior to virtual screening approaches that targetted the SAM binding site of HMTs, the binding modes of the cofactor, SAM or SAH, were visualized from the protein crystal structures of HMTs using the programs AutoDockTools and PyMOL. Although these three enzymes, G9a, GLP, and SUV39H2, mediate the same H3K9-specific methylation, the shape of each binding pocket occupied by SAM or SAH looks different in the complex structures. Furthermore, even in the same enzyme (GLP in complex with SAH), binding of the different substrates (without H3K9 peptide, with H3K9 monomethyl and dimethyl peptides) induced a change in the shape of the SAM binding pocket. In the GLP complex with H3K9me1 (3HNA) or H3K9me2 (2RFI) peptides, the entrance of the SAM binding pockets was narrower than that of the complex without H3K9 peptide (2IGQ) (Figure 10.5). In subsequent studies, ligand similarity and subsequent virtual docking 

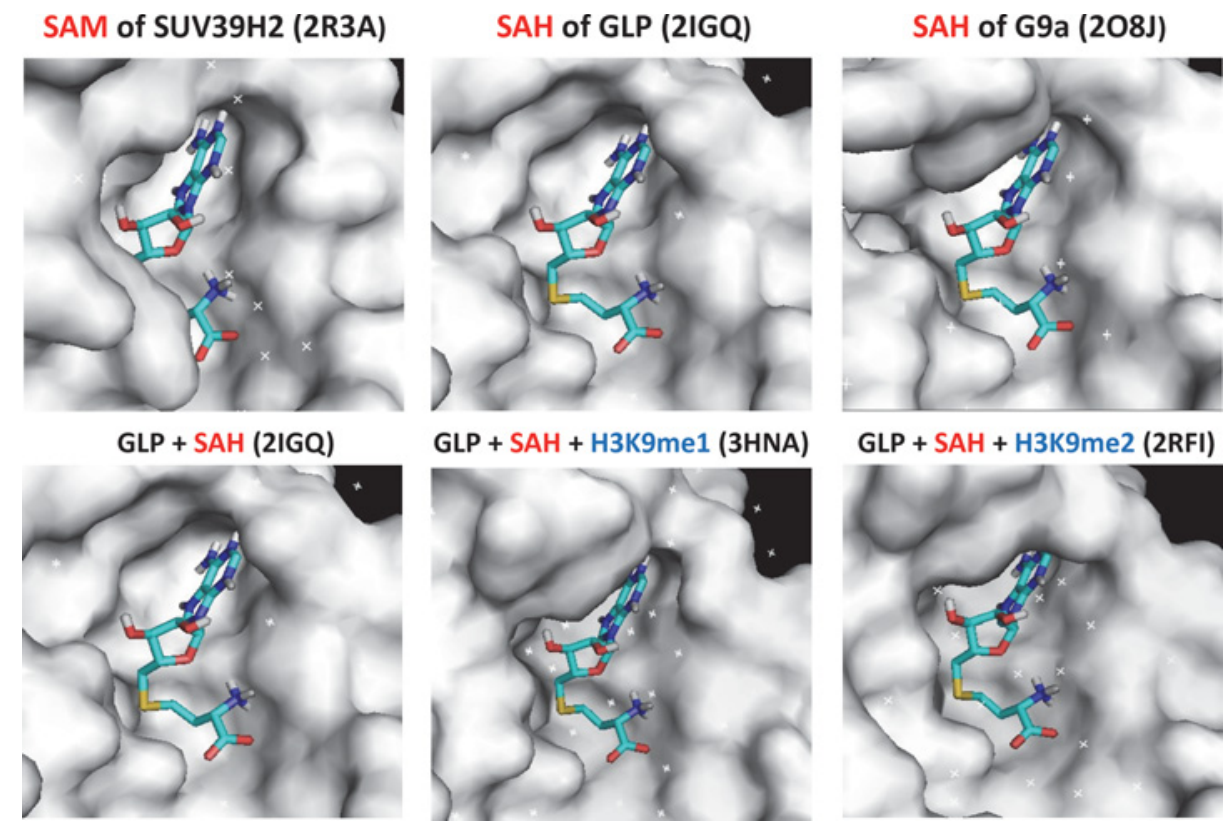

Figure 10.5 Surface maps of the SAM binding pocket of H3K9 methyltransferases in complex with SAM or SAH. The PDB ID of each complex is shown in parentheses

screenings with the five protein structures of three H3K9 methyltransferases were performed in the same way as the DNMT1 inhibitor screening approach. Docking results were evaluated by a comparison of the top 100 "hit" compounds identified from each docking to present a variability of SAM binding pockets of the structures. Surprisingly, about $60 \%$ of the compounds were unique among the three different HMTs, and over $60 \%$ were unique between even the same enzymes: GLPs with different types of substrate bound (Figure 10.6). These observations suggest that even though HMTs use a universal methyl
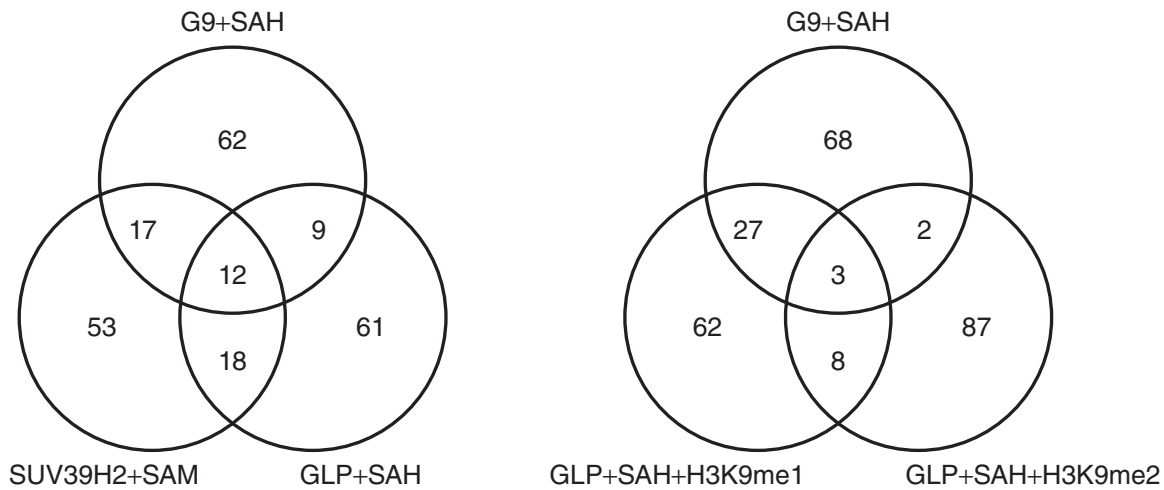

Figure 10.6 Comparison of each top-100 compound derived from virtual docking. Names of the enzyme, cofactor, and substrate are shown around the circles 
donor SAM as a cofactor, the SAM binding sites of HMTs with a high degree of conservation can be targeted for the development of specific inhibitors to each HMT. Furthermore, it might be possible to develop inhibitors with specificity to the mono-, di-, or trimethyl activities of each HMT. To test this possibility, over 300 compounds selected from five virtual dockings were obtained and tested using G9a ELISA. The ELISA scheme is shown in Figure 10.7a. Briefly, the biotinylated and unmethylated H3K9 peptides were methylated by purified human G9a in the presence of $10 \mu \mathrm{M}$ of each test compound. Monomethylated $\mathrm{H} 3 \mathrm{~K} 9$ peptides were recognized by antihistone $\mathrm{H} 3 \mathrm{~K} 9$ monomethyl-specific monoclonal antibodies and followed by HRP-labeled secondary antibody and HRP substrate reactions. Signal intensity on the plate was measured by a luminometer. To investigate H3K9 dimethyl and trimethyl inhibition of G9a in the presence of each test compound, we employed (i) the biotinylated $\mathrm{H} 3 \mathrm{~K} 9$ monomethyl peptide and antidimethyl-specific antibody and (ii) anti-H3K9 dimethyl peptide and trimethyl-specific antibody, respectively. From the initial screening, 25 compounds were selected based on the criterion of less than 70\% G9a activity. This initial screening result shows that it is possible to develop specific inhibitors targeting the SAM binding site of each HMT.

\subsubsection{SAM Competitive Assay}

To confirm direct competition of SAM by 25 hit compounds, we used a fluorescence polarization-based SAM GLP Screener Assay (Cayman), or modified versions of the assay developed in-house for other methyltransferases such as G9a and DNMT1. The fluorescence polarization assay is based upon a proprietary small-molecule fluorescent probe that binds to the SAM binding pocket. Binding of the small-molecule probe induces an increase in fluorescence polarization. When the SAM-binding-site probe is bound, the probe displays a dramatic increase in fluorescence polarization as compared to the free probe. Small molecules such as the positive control compound sinefungin compete directly with the SAM-binding-site probe for SAM binding on the enzyme. Displacement of the SAMbinding-site probe by a small molecule causes a detectable loss of fluorescence polarization in the assay in a concentration-dependent manner. Figure 10.8 demonstrates a loss of fluorescence polarization for several compounds identified by targeting the SAM binding pocket for GLP (c) and G9a (d).

\subsubsection{SAM Binding Site is Unique and Selective across Multiple Epigenetic Targets}

There are over 500 kinases encoded in the human genome that utilize ATP as a substrate, and they all share a high degree of conservation in their ATP binding sites. Despite this, highly selective small molecules with favorable pharmaceutical properties have been developed that selectively target the ATP binding sites of particular kinases, which has allowed them to serve as important anticancer drugs. Similarly, all methyltransferases use SAM as a universal methyl donor. Even though all methyltransferases share a high degree of conservation in the SAM binding site, we believe that highly selective PMT inhibitors can be developed by targeting the SAM binding site landscape as we have described. This idea is supported by other evidence. Fluorescence polarization assays were performed with G9a and GLP methyltransferases in the presence of each histone substrate (H3K9 mono-, di-, and trimethyl peptides). Figure 10.9 shows that binding of each substrate induced a 


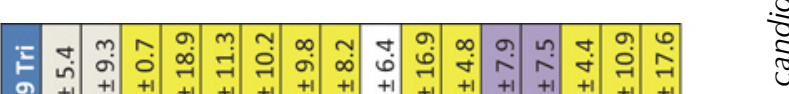

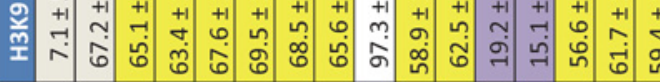

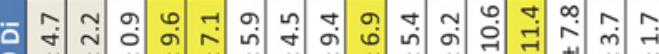

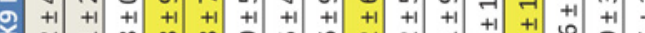

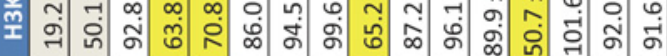

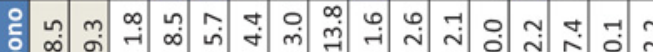

E

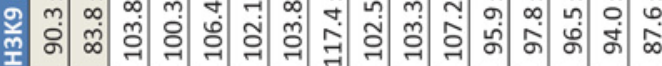

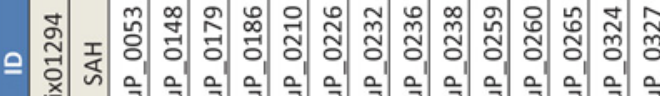

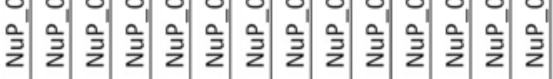

a
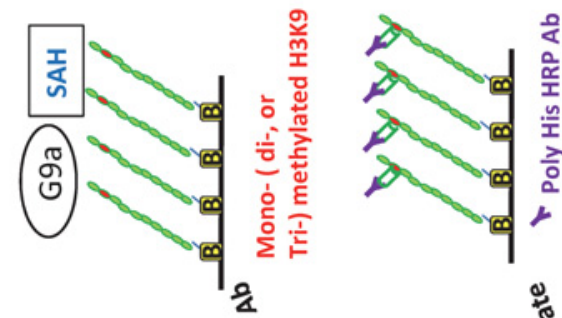

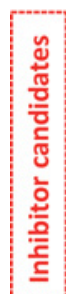

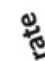



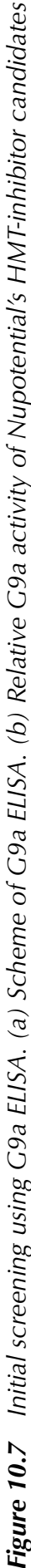




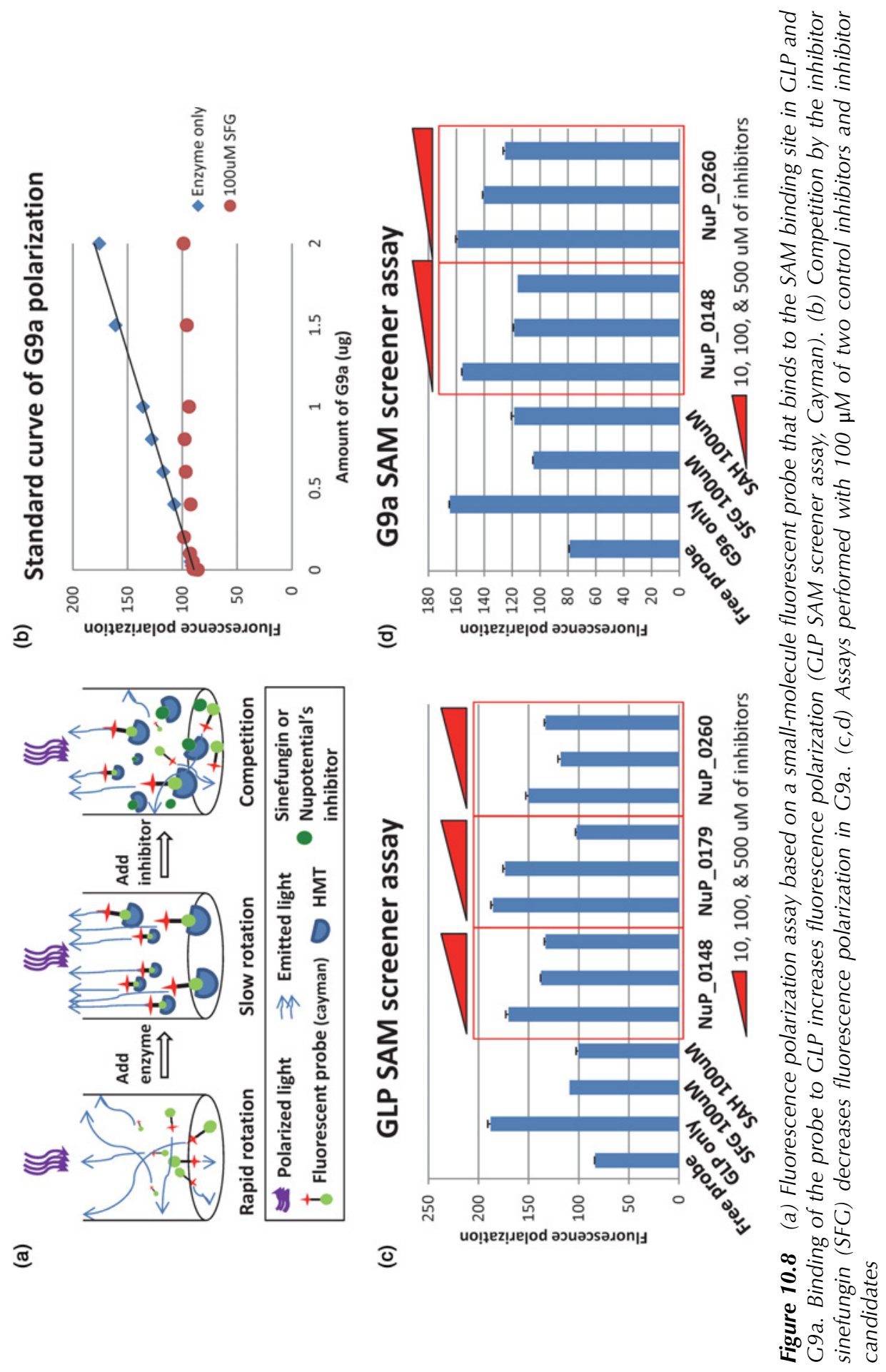




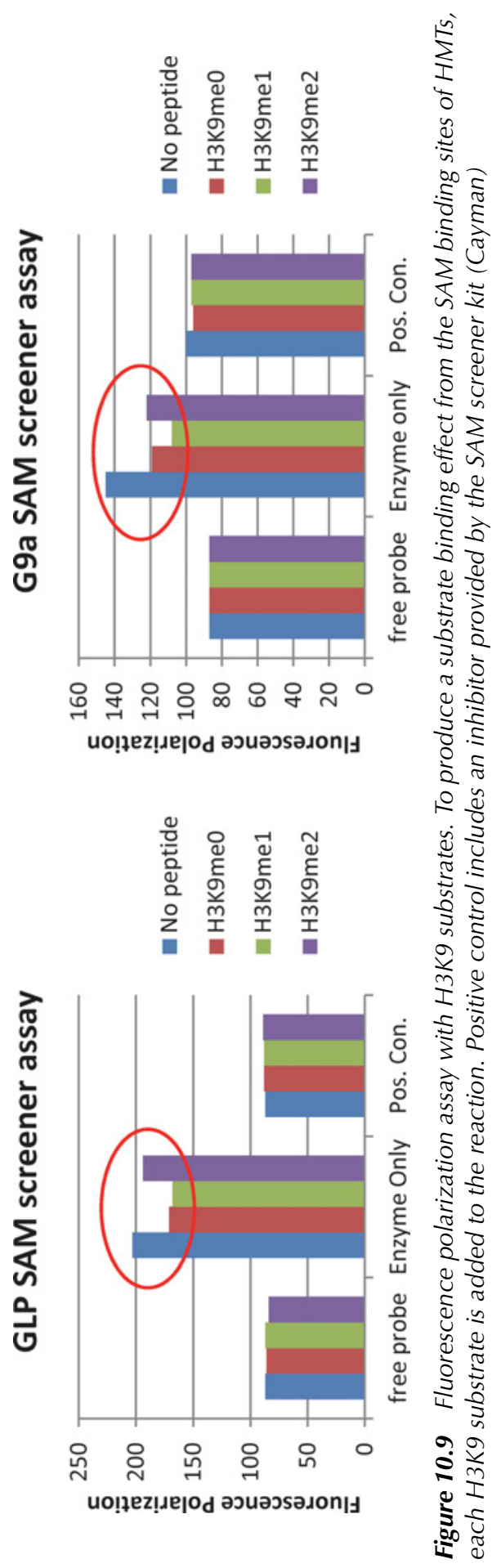


change of fluorescence polarization signals (red circles), which means that the different types of substrate binding induced a conformational change of the SAM binding site in GLP and G9a.

\subsection{Conclusion}

There is experimental evidence that small-moleclue inhibitors can be developed for methyltransferases based on recent advances in our knowledge of their biochemistry, substrate selectivity, and 3D structure. The generation of a sufficient supply of stem cells that can be differentiated into specific tissues would advance the development of regenerative and cell-based therapies. Considerable efforts are now underway to discover small-molecule substitutes for transcription factors in order to make the reprogramming process more efficient. Identification of inhibitors that are increasingly target-selective should help to realize the production of iPSCs without the need for transgenes. In addition, there is a developing body of evidence that methyltransferases have critical pathogenic roles in human diseases, including cancer, and small-molecule inhibitors are likely to be effective therapuetics. Our efforts have focused on the identification and development of methyltransferase inhibitors using a virtual screening approach that exploits the SAM binding sites of these enzymes.

\section{References}

(1) Yu, J.Y., Hu, K.J., Smuga-Otto, K. et al. (2009) Human induced pluripotent stem cells free of vector and transgene se- quences. Science, 10, 797-801. doi: 10.1126/science.1172482.

(2) Sommer, C.A., Stadtfeld, M., Murphy, G.J. et al. (2009) Induced pluripotent stem cell generation using a single lentiviral stem cell cassette. Stem Cells, 27, 543-549. doi: 10.1634/stemcells.20081075 .

(3) Huangfu, D.W., Maehr, R., Guo, W.J. et al. (2008) Induction of pluripotent stem cells by defined factors is greatly improved by small-molecule compounds. Nature Biotechnology, 26, 795-797.

(4) Okita, K., Nakagawa, M., Hong, H. et al. (2008) Generation of mouse induced pluri- potent stem cells without viral vectors. Science, 322, 949-953. doi: 10.1126/science.1164270.

(5) Warren, L., Manos, P.D., Ahfeldt, T. et al. (2010) Highly efficient reprogramming to pluripotency and directed differentiation of human cells with synthetic modified mRNA. Cell Stem Cell, 7, 618-630. doi: 10.1016/j.stem.2010.08.012.

(6) Yakubov, E., Rechavi, G., Rozenblatt, S., and Givol, D. (2010) Reprogramming of human fibroblasts to pluripotent stem cells using mRNA of four transcription factors. Biochemical and Biophysical Research Communications, 394, 189-193. doi: 10.1016/j.bbrc.2010.02.150.

(7) Anokye-Danso, F., Trivedi, C.M., Juhr, D. et al. (2011) Highly efficient miRNA-mediated reprogramming of mouse and human somatic cells to pluri- potency. Cell Stem Cell, 8, 376-388. doi: 10.1016/j.stem.2011.03.001.

(8) Woltjen, K., Michael, I.P., Mohseni, P. et al. (2009) piggyBac transposition reprograms fibroblasts to induced pluripotent stem cells. Nature, 458, 766-770.

(9) Kaji, K., Norrby, K., Paca, A. et al. (2009) Virus-Free induction of pluripotency and subsequent excision of reprogramming factors. Nature, 458, 771-775.

(10) Kim, D., Kim, C.-H, Moon, J.-I. et al. (2009) Generation of human induced pluripotent stem cells by direct delivery of reprogramming proteins. Cell Stem Cell, 4, 472-476. doi: 10.1016/j.stem.2009.05.005

(11) Zhou, H.Y., Wu, S.L., Joo, J.Y. et al. (2009) Generation of induced pluripotent stem cells using recombinant proteins. Cell Stem Cell, 4, 381-384. 
(12) Mikkelsen, T.S., Hanna, J., Zhang, X. et al. (2008) Dissecting direct reprogramming through integrative genomic analysis. Nature, 454 (7200), 49-55. doi: 10.1038/nature07056. Epub 2008 May 28.

(13) Okita K., Ichisaka T., and Yamanaka S. (2007) Generation of germline-competent induced pluripotent stem cells. Nature, 448 (7151), 313-317. Epub 2007 Jun 6.

(14) Silva, J., Barrandon, O., Nichols, J. et al. (2008) Promotion of reprogramming to ground state pluripotency by signal inhibition. PLoS Biology, 6 (10), e253. doi: 10.1371/journal.pbio.0060253.

(15) Rim, J.S., Strickler, K.L., Barnes, C.W. et al. (2012) Temporal epigenetic modifications differentially regulate ES cell-like colony formation and maturation. Stem Cell Discovery, 2 (2), 45-57. doi: 10.4236/scd.2012.22008

(16) Turnbull, J.F. and Adams, R.L. (1976) DNA methylase: purification from ascites cells and the effect of various DNA substrates on its activity. Nucleic Acids Research, 3 (3), 677-695.

(17) Pradhan, S. and Esteve, P.O. (2003) Mammalian DNA (cytosine-5) methyltransferases and their expression. Clinical Immunology, 109 (1), 6-16.

(18) Razin, A. and Riggs, A.D. (1980) DNA methylation and gene function. Science, 210 (4470), 604-610.

(19) Xie, S., Wang, Z., Okano, M. et al. (1999) Cloning, expression and chromosome locations of the human DNMT3 gene family. Gene, 236 (1), 87-95.

(20) Gravina, G.L., Festuccia, C., Marampon, F. et al. (2010) Biological rationale for the use of DNA methyltransferase inhibitors as new strategy for modulation of tumor response to chemotherapy and radiation. Molecular Cancer [Electronic Resource], 9, 305.

(21) Stresemann, C. and Lyko, F. (2008) ModesofactionoftheDNAmethyltransferase inhibitors azacytidineanddecitabine. International Journal of Cancer, 123, 8-13

(22) Silverman, L.R., Demakos, E.P., Peterson, B.L. et al. (2002) Randomized controlled trial of azacitidine in patients with the myelodysplastic syndrome: a study of the cancer and leukemia group B. Journal of Clinical Oncology, 20, 2429-2440.

(23) Kantarjian, H., Issa, J.P., Rosenfeld, C.S. et al. (2006.) Decitabine improves patient outcomes in myelodysplastic syndromes: results of a phase III randomized study. Cancer, 106, 1794-1803.

(24) Raj, K. and Mufti, G.J. (2006) Azacytidine $\left(\right.$ Vidaza $\left.^{\circledR}\right)$ in the treatment of myelodysplastic syndromes. Therapeutics and Clinical Risk Management, 2 (4), 377-388.

(25) Silverman, L.R., Demakos, E.P., Peterson, B.L. et al. (2002) Randomized controlled trial of azacitidine in patients with the myelodysplastic syndrome: a study of the cancer and leukemia group B. Journal of Clinical Oncology, 20, 2429-2440.

(26) Saba, H.I. (2007) Decitabine in the treatment of myelodysplastic syndromes. Therapeutics and Clinical Risk Management, 3 (5), 807-817.

(27) Kuck, D., Singh, N., Lyko, F., and Medina-Franco, J.L. (2010) Novel and selective DNA methyltransferase inhibitors: Docking-based virtual screening and experimental evaluation. Bioorganic \& Medicinal Chemistry, 18 (2), 822-829.

(28) Siedlecki, P., Garcia, B.R., Musch, T. et al. (2006) Discovery of two novel, small-molecule inhibitors of DNA methylation. Journal of Medicinal Chemistry, 49 (2), 678-683.

(29) Martin, C. and Zhang, Y. (2005) The diverse functions of histone lysine methylation. Nature Reviews Molecular Cell Biology, 6 (11), 838-849.

(30) Trojer, P., Li, G., Sims, R.J. 3rd et al. (2007) L3MBTL1, a histone-methylation-dependent chromatin lock. Cell, 129 (5), 915-928.

(31) Nielsen, P.R., Nietlispach, D., Mott, H.R. et al. (2002) Structure of the HP1 chromodomain bound to histone H3 methylated at lysine 9. Nature, 416 (6876), 103-107. Epub 2002 Feb 20.

(32) Bissinger, E.-A., Heinke, R., Sippl, W., and Jung, M. (2010) Targeting epigenetic modifiers: inhibitors of histone methyltransferases. Medicinal Chemistry Communications, 1, 114-124.

(33) Huang, J., Sengupta, R., Espejo, A.B. et al. (2007) p53 is regulated by the lysine demethylase LSD1. Nature, $\mathbf{4 4 9}$ (7158), 105-108.

(34) Shi, X., Kachirskaia, I., Yamaguchi, H. et al. (2007) Modulation of p53 function by SET8mediated methylation at lysine 382. Molecular Cell, 27 (4), 636-646.

(35) Chuikov, S., Kurash, J.K., Wilson, J.R. et al. (2004) Regulation of p53 activity through lysine methylation. Nature, 432 (7015), 353-360. Epub 2004 Nov 3. 
(36) Huang, J., Dorsey, J., Chuikov, S. et al. (2010) G9a and Glp methylate lysine 373 in the tumor suppressor p53. Journal of Biological Chemistry, 285 (13), 9636-9641. doi: 10.1074/jbc.M109.062588. Epub 2010 Jan 29.

(37) Kondo, Y., Shen, L., Ahmed, S. et al. (2008) Downregulation of histone H3 lysine 9 methyltransferase G9a induces centrosome disruption and chromosome instability in cancer cells. PLoS One, 3 (4), e2037. doi: 10.1371/journal.pone.0002037.

(38) Kondo, Y., Shen, L., Suzuki, S. et al. (2007) Alterations of DNA methylation and histone modifications contribute to gene silencing in hepatocellular carcinomas. Hepatology Research, 37 (11), 974-983. Epub 2007 Jun 20.

(39) Maze, I., Covington, H.E. 3rd, Dietz, D.M. et al. (2010) Essential role of the histone methyltransferase G9a in cocaine-induced plasticity. Science, 327 (5962), 213-216. doi: 10.1126/science.1179438.

(40) Schaefer, A., Sampath, S.C., Intrator, A. et al. (2009) Control of cognition and adaptive behavior by the GLP/G9a epigenetic suppressor complex. Neuron, 64 (5), 678-691. doi: 10.1016/j.neuron.2009.11.019.

(41) Shi, Y., Do, J.T., Desponts, C. et al. (2008) A combined chemical and genetic approach for the generation of induced pluripotent stem cells. Cell Stem Cell, 2 (6), 525-528. doi: 10.1016/j.stem.2008.05.011.

(42) Feldman, N., Gerson, A., Fang, J. et al. (2006) G9a-mediated irreversible epigenetic inactivation of Oct-3/4 during early embryogenesis. Nature Cell Biology, 8 (2), 188-194. Epub 2006 Jan 15.

(43) Epsztejn-Litman, S., Feldman, N., Abu-Remaileh, M. et al. (2008) De novo DNA methylation promoted by G9a prevents reprogramming of embryonically silenced genes. Nature Structural \& Molecular Biology, 15 (11), 1176-1183. doi: 10.1038/nsmb.1476. Epub 2008 Oct 26.

(44) Shi, Y., Desponts, C., Do, J.T. et al. (2008) Induction of pluripotent stem cells from mouse embryonic fibroblasts by Oct4 and Klf4 with small-molecule compounds. Cell Stem Cell, 3 (5), 568-574. doi: 10.1016/j.stem.2008.10.004

(45) Onder, T.T., Kara, N., Cherry, A. et al. (2012) Chromatin-modifying enzymes as modulators of reprogramming. Nature, 483 (7391), 598-602. doi: 10.1038/nature10953

(46) Vedel, M., Lawrence, F., Robert-Gero, M., and Lederer, E. (1978) The antifungal antibiotic sinefungin as a very active inhibitor of methyltransferases and of the transformation of chick embryo fibroblasts by Rous sarcoma virus. Biochemical and Biophysical Research Communications, $\mathbf{8 5}$ (1), 371-376.

(47) Qian, C. and Zhou, M.M. (2006) SET domain protein lysine methyltransferases: Structure, specificity and catalysis. Molecular and Cellular Life Sciences, 63 (23), 2755-2763.

(48) Kubicek, S., O'Sullivan, R.J., August, E.M. et al. (2007) Reversal of H3K9me2 by a smallmolecule inhibitor for the G9a histone methyltransferase. Molecular Cell, 25 (3), 473-481.

(49) Liu, F., Chen, X., Allali-Hassani, A. et al. (2009) Discovery of a 2,4-diamino-7aminoalkoxyquinazoline as a potent and selective inhibitor of histone lysine methyltransferase G9a. Journal of Medicinal Chemistry, 52 (24), 7950-7953. doi: 10.1021/jm901543m

(50) Trott, O. and Olson, A.J. (2010) AutoDock Vina: improving the speed and accuracy of docking with a new scoring function, efficient optimization, and multithreading. Journal of Computational Chemistry, 31 (2), 455-461.

(51) DeLano, W.L. (2008.) The PyMOL Molecular Graphics System, DeLano Scientific LLC, Palo Alto, CA. 


\title{
11
}

\section{Biomaterials for Directed Differentiation}

\author{
Xintong Wang ${ }^{1}$, Angela L. Zachman ${ }^{1}$, Simon Maltais ${ }^{2}$, and Hak-Joon Sung ${ }^{3}$ \\ ${ }^{1}$ Department of Biomedical Engineering, Vanderbilt University, USA \\ ${ }^{2}$ Department of Cardiac Surgery, Vanderbilt University, USA \\ ${ }^{3}$ Department of Biomedical Engineering, School of Medicine, Vanderbilt University, USA
}

\section{Box 11.1 Abbreviations and Acronyms}

ALP alkaline phosphatease

BMP bone morphogenic protein

EB embryoid body

ECM extracellular matrix

EPC endothelial progenitor cell

ESC embryonic stem cell

FAK focal adhesion kinase

iPSC induced pluripotent stem cell

MSC mesenchymal stem cell

NSC neural stem cell

NPC neural progenitor cell

PCL poly( $\varepsilon$-caprolactone)

PDGF platelet-derived growth factor

PDL poly(D-lysine) 


$\begin{array}{ll}\text { PDMS } & \text { poly(dimethylsiloxane) } \\ \text { PEG } & \text { poly(ethylene glycol) } \\ \text { PEGDA } & \text { poly(ethylene glycol) diacrylate } \\ \text { PGA } & \text { poly(glycolic acid) } \\ \text { PHEMA } & \text { poly(2-hydroxyethyl methacrylate) } \\ \text { PLA } & \text { poly(lactic acid) } \\ \text { PLLA } & \text { poly(L-lactic acid) } \\ \text { PLGA } & \text { poly(lactic-co-glycolic acid) } \\ \text { RGD } & \text { arginine-glycine-aspartic acid } \\ \text { SMC } & \text { smooth muscle cell } \\ \text { VEGF } & \text { vascular endothelial growth factor } \\ \text { TGF } & \text { transforming growth factor } \\ \text { UC } & \text { umbilical cord }\end{array}$

\subsection{Introduction}

Stem cells and progenitor cells reside in a complex microenvironment consisting of multiple types of extracellular matrix (ECM) components, including matrix proteins, proteoglycans, hyaluronic acid, and growth factors deposited on or tethered to ECM. These ECM components present a great variety of ligands and provide mechanical and topographical cues to stem cells. The extracellular cues are sensed by stem cells and trigger intracellular signaling pathways, thereby determining the self-renewal or differentiation of the stem cells. Working closely with surrounding cells through direct cell-cell contact or via paracrine pathways, these extracellular cues form "niches" and help to define stem-cell fate.

As an important field of regenerative medicine, tissue engineering is becoming increasingly attractive with the fast-paced development of biomaterials. Stem cells are in a unique position to satisfy the needs of regenerative medicine due to their potential to differentiate into multiple lineages. The tremendous progress in stem-cell research provides greater opportunities to regenerate tissues and organs than we could ever imagine. In addition to using biomaterial scaffolds to improve the growth and proliferation of fully differentiated somatic cells, stem cell-based regenerative medicine requires the scaffolds to support and control the differentiation of stem cells. Therefore, understanding the interactions between stem cells and biomaterials ("structure-function relationships") is critical to the design and fabrication of functional products. On the other hand, elucidating how ECM ligands and the physical properties of substrates regulate stem-cell fate will provide insight into the development of new biomaterials for successful tissue regeneration.

In this chapter, we introduce the roles of ECM-derived biomaterials in regulating stemcell differentiation, which include Matrigel, collagen, laminin, and fibrin, followed by the applications of typical non-ECM-derived natural and synthetic biomaterials in the control of stem-cell fate. The progress of this area within the past 10 years is discussed. We aim to provide a comprehensive but concise review of the biomaterials being applied to direct stem-cell differentiation for regenerative medicine. 


\subsection{Natural Biomaterials}

Natural biomaterials, including both ECM- and non-ECM-derived components, have been used to produce scaffolds for tissue regeneration. Many ECM components, such as collagen, fibronectin, hyaluronic acid, proteoglycans, and hydroxyapatite (HA), are widely applied. Non-ECM components, such as chitosan, alginate, and silk, have also been used to fabricate scaffolds. The advantage of using these natural materials is that they mimic the physiological environment that stem cells encounter in vivo by providing ligands similar to those found in the stem cell niche. However, the disadvantages of using natural materials in regenerative medicine include: (i) the difficulty in controlling the chemical and physical properties of the material for different applications; (ii) batch-to-batch variation; (iii) the possibility of pathogen contamination; and (iv) in vivo immunogenicity with materials of animal origin. In spite of these drawbacks, natural materials are still being extensively used in tissue engineering due to their similarity to the stem cell niche and the ease with which they can be obtained from commercial sources, with minimal manipulation needed before application.

\subsubsection{ECM-Derived Materials}

\subsubsection{Matrigel}

Matrigel is a superior substrate with which to maintain and differentiate stem cells. It is derived from murine sarcoma cells and consists of type IV collagen, laminin, a small amount of heparan sulfate proteoglycan, and growth factors [1]. It is commercialized by BD Biosciences. Matrigel has been used as a feeder-free substrate for a wide selection of stem cells, especially the pluripotent stem cells, such as embryonic stem cells (ESCs) and induced pluripotent stem cells (iPSCs) [2-19]. It has also been used in the differentiation of pluripotent stem cells to all three germ lines. For example, mouse embryonic stem cells (mESCs) were grown on Matrigel and were differentiated to thyroid follicular cells [18]; pancreatic insulin-producing cells were obtained from ESC-derived endoderms on Matrigel $[3,8]$; hepatic cells were differentiated from ESCs that were encapsulated in Matrigel and loaded into a poly(lactic-co-glycolic acid) (PLGA) scaffold [10]. Interestingly, Matrigel and type I collagen supported the hepatic differentiation of human embryonic stem cells (hESCs), but hESCs could not attach to laminin, even though laminin is one of the major components of Matrigel [12]. The growth factor-reduced Matrigel efficiently supported survival and neurite outgrowth of neural precursor cells in vitro, unlike collagen and laminin. It also promoted the proliferation and differentiation of neural precursor cells in vivo, resulting in an increased number of neurons through suppression of the inflammatory response [20].

Besides supporting pluripotent stem cells to differentiate along the endoderm direction, Matrigel also aids in the differentiation of pluripotent cells to mesodermal lineages, such as vascular [4, 9, 14, 16], cardiac [5, 19], and mesenchymal stromal cells [21]. Matrigel has traditionally been used as a substrate to demonstrate the vascular-tube formation of stem cells. Moreover, Nakagami et al. have reported that sprouting blood vessels could be differentiated from murine ESC-derived embryoid bodies (EBs) on Matrigel, but not on collagen or gelatin [16]. Remarkably, a Matrigel sandwich method was developed to 
differentiate human induced pluripotent stem cells (hiPSCs) to cardiomyocytes. By seeding iPSCs on Matrigel and subsequently overlaying another layer of Matrigel on top, this method promoted epithelial-mesenchymal transition (EMT) and substantially increased the differentiation of iPSCs to cardiomyocytes in vitro with high efficiency ( 98\%). A pool of mixed cardiomyocytes, including embryonic nodal, atrial, and ventricular cardiomycoytes, was obtained after 30 days [19]. Although Matrigel is a wonderful substrate for supporting the growth and differentiation of many types of stem cell, its complex and heterogeneous composition makes isolating the effect of individual components very challenging, indicating that biomaterials with a simple composition are required to better predict the stem-cell fate.

\subsubsection{Fibrin}

Fibrin gels or fibrin networks are created during the natural wound-healing process when fibrinogen is converted to fibrin by thrombin and the fibrin strands are then crosslinked in the presence of factor XIIIa. Thus, fibrin is considered another type of natural material that can be applied to support stem-cell differentiation.

The effects of fibrin on endothelial progenitor cell (EPC) growth and differentiation were studied at different fibrinogen-thrombin ratios. The viability, differentiation, and angiogenic capabilities of EPCs were evaluated and compared to EPCs grown on fibronectin. Fibrin formed a porous network in vitro, and the stiffness significantly influenced the growth of EPCs. Cell viability was found to be higher on fibrin than on fibronectin. EPCs on fibrin differentiated to vascular cells and released cytokines to recruit other cells, indicating that fibrin could serve as a suitable matrix for EPC growth, differentiation, and angiogenesis [22]. In another study, fibrin was mixed with fibronectin, gelatin, and growth factors, which successfully induced the differentiation of EPCs and smooth muscle progenitor cells to endothelial and smooth muscle cells (SMCs), respectively [23].

Fibrin gels can be used in bone regeneration. Osteogenic differentiation of human mesenchymal stem cells (hMSC) seeded in a fibrin sealant was analyzed by altering the fibrin gel composition. Cell proliferation was higher with a low concentration of fibrinogen, but when the fibrinogen concentration was increased the alkaline phosphatease (ALP) activity, bone sialoprotein gene expression, and small nodules of mineralization were enhanced after 3 weeks. However, there was no significant increase in osteocalcin expression after 4 weeks. These results suggest the osteogenic differentiation of hMSCs is dependent on the fibrinogen-thrombin ratio of the fibrin sealant, but hMSCs did not fully differentiate to mature osteoblasts [24]. Autologous platelet-rich fibrin glue containing canine bone marrow-derived mesenchymal stem cells (BM-MSCs) was used to regenerate bone defects with or without MEDPOR. Radiographic analysis 4 months post-transplantation showed an average of $72.8 \pm 8.02 \%$ new bone formation with MSC-containing fibrin glue guided by MEDPOR and a $53.34 \pm 6.87 \%$ new bone formation in the group with MSC-containing fibrin glue without MEDPOR, compared to $15.14 \pm 2.37 \%$ in a control group with fibrin gel alone. Histological analysis demonstrated the defect was repaired by typical bone tissue in MSC-containing fibrin glue both with and without MEDPOR, whereas only minimal bone formation was observed in the control group, showing the critical role of fibrin in supporting osteogenesis by MSCs [25]. Growth factor-loaded particles can be mixed in fibrin scaffolds to direct osteogenic differentiation via controlled release of growth factors. For example, 
a system combining fibrin and bone morphogenic protein 2 (BMP2)-loaded nanocarriers was applied for the osteogenic differentiation of hMSCs. The controlled release of BMP2 by nanocarriers significantly promoted the osteogenic differentiation of hMSCs embedded in fibrin scaffolds as compared to non-BMP2 scaffold or BMP2-loaded fibrin constructs without nanoparticles [26].

Porcine MSCs were able to differentiate into adipogenic, osteogenic, and chondrogenic lineages when cultured on blood-derived autologous fibrin scaffolds [27]. Interestingly, fibrin microthreads were developed to embed hMSCs, and these could be used as a suture to enhance the efficiency and localization of stem-cell delivery. The cells were viable and proliferative in microthreads. Moreover, the hMSCs in the microthreads retained their multipotency and could differentiate to adipocytes and osteocytes, providing a potential platform for the efficient delivery of stem cells in vivo [28].

Fibrin scaffolds have also been applied in neurogenesis of stem cells. A fibrin scaffold was produced to release vascular endothelial growth factor (VEGF) in order to control the migration of murine neural stem cells (NSCs) (C17.2) for neuroregeneration. The stem cells embedded in collagen hydrogels were printed close to the VEGF-releasing fibrin gel. The C17.2 cells printed within $1 \mathrm{~mm}$ of the border of fibrin gel changed their morphology in the presence of VEGF and migrated toward the fibrin gel for a total distance of $102.4 \pm 76.1 \mu \mathrm{m}$ over 3 days [29]. Other growth factors were also incorporated in fibrin scaffolds to direct the differentiation of ESC-derived neural progenitor cells. Examples include neurotrophin 3 (NT3), Sonic hedgehog (SHH), and platelet-derived growth factor (PDGF). Mouse EBs containing neural progenitors were seeded in these fibrin scaffolds. The simultaneous delivery of NT3 and PDGF from fibrin increased the fraction of neural progenitors, neurons, and oligodendrocytes and decreased the fraction of astrocytes compared to EBs in fibrin scaffolds without growth factors [30].

\subsubsection{Collagen}

As the most abundant ECM protein, collagen favors stem-cell commitment toward many lineages. Its excellent and adjustable mechanical properties make it ideal for directing the differentiation of stem cells to myogenic lineages. The effects of collagen I and IV on mouse BM-MSC differentiation to SMCs were evaluated [31,32]. BM-MSCs cultured on collagen I and IV reduced the expression of SMC markers such as smooth muscle actin (SMA) and myosin heavy chain (MHC) compared to cells on tissue culture plastic. Growth factors including transforming growth factor $\beta 1$ (TGF $\beta 1$ ) and PDGF-BB enhanced SMC differentiation on collagen. These results suggest that, unlike plastic, collagen tends to maintain the undifferentiated state of BM-MSCs in the absence of exogenous growth factors [31]. Human BM-MSCs seeded on collagen-coated silicon membranes underwent myogenic differentiation with the addition of insulin-like growth factor 1 (IGF1). The combination of mechanical loading and growth-factor treatment led to enhanced expression of myogenic markers such as Myf5, MyoD, MyoG, and Myf6, indicating the supportive role of collagen in myogenesis of MSCs [33]. Type I collagen was micropatterned on a polyacrylamide gel with a stiffness of $10.2 \mathrm{kPa}$, which efficiently modulated elongated focal adhesion complexes of hMSCs, and in turn preferentially recruited the $\beta 3$ integrin cluster, regulated RhoA downstream signaling, and induced specific myogenic differentiation at both transcriptional and translational levels [34]. 
To mimic the stem cell niche of collagen-rich tissues such as cartilage and bone, collagen has been combined with other structural biomaterials and growth factors to direct stemcell fate toward chondrogenic and osteogenic lineages. Human umbilical cord-derived MSCs (UC-MSCs) seeded in collagen hydrogels differentiated to chondrogenic cells after exposure to chondrogenic factors, as characterized by increased expression of collagen II, aggrecan, cartilage oligomeric matrix protein, and SOX9. Thus collagen hydrogels provide an appropriate 3D environment for the promotion of chondrogenic differentiation of UC-MSCs for cartilage regeneration [35]. In another study, when UC-MSCs were grown on poly( $\varepsilon$-caprolactone) (PCL)/collagen nanoscaffolds for 3 weeks cell proliferation and chondrogenesis were found to be higher than in human BM-MSCs. The authors also demonstrated that basic fibroblast growth factor (bFGF) in culture medium played a crucial role in chondrogenesis [36]. Other factors, such as mechanical stimulation and oxygen tension, were revealed to be important in directing the tenogenic lineage commitment of adipose tissue-derived stem cells on type I collagen gel [37].

Collagen-glycosaminoglycan (chondroitin sulfate or hyaluronic acid) scaffolds were applied to assay the effects of stiffness and composition on MSC differentiation without the addition of differentiation supplements. The scaffolds with lower stiffness $(0.5 \mathrm{kPa})$ favored the chondrogenic differentiation of MSCs, while scaffolds with higher stiffness $(1.5 \mathrm{kPa})$ directed cells to osteogenic differentiation, as revealed by the expression of SOX9 and RUNX2, respectively. Besides scaffold stiffness, the composition also influenced MSC differentiation. Upregulation of SOX9 and RUNX2 were observed with collagen-hyaluronic acid and collagen-chondroitin sulfate scaffolds, respectively, indicating hyaluronic acid is more efficient in chondrogenic differentiation and chondroitin sulfate is more potent in inducing osteogenesis. More importantly, these results demonstrate that the differentiation of MSCs can be controlled by intrinsic properties of the substrate in the absence of differentiation supplements [38]. Other than stiffness and composition, 3D collagen hydrogels were able to support the differentiation of MSCs to osteoblasts with osteoinductive supplements. Osteocalcin expression and calcium deposition were significantly higher in 3D hydrogel than in 2D culture [39]. The morphology of osteoblasts differentiated from MSCs in 3D collagen cultures was similar to that of osteoblasts in vivo, as compared to cells in $2 \mathrm{D}$ collagen cultures [40].

Matrigel has been shown to promote cardiogenesis by many groups. As one of the major components of Matrigel, collagen IV may be able to support cardiac differentiation of stem cells. In one study, collagen IV was applied in directing mouse ESCs to cardiac cells. It was demonstrated the fraction of $\mathrm{Flk}^{+}$cells was higher on collagen IV than on fibronectin, laminin, or vitronectin in $2 \mathrm{D}$ culture. However, vitronectin induced the most $\mathrm{Flk}^{+}$cells among all these substrates when tested in 3D culture system [41]. The result was confirmed by another study comparing cardiac differentiation of hESC-derived EBs on different ECM-derived matrices under hypoxia. Collagen IV and gelatin increased the overall number of beating EBs and enhanced the cardiac troponin T (cTNT) expression compared to fibronectin. These results revealed that collagen IV could potentially serve as a substrate for cardiogenesis [42]. In addition to collagen IV used in cardiogenesis ex vivo, the interaction between type I collagen and $\beta 1$ integrin has been suggested to be critical for the growth and cardiac differentiation of mouse iPSC-derived EBs, indicating that collagen I may be a potential substrate for cardiogenesis as well [43].

Human BM-MSCs can be directed to the epidermal lineage on electrospun collagen/ poly(L-lactic acid)-co-PCL (collagan/poly(L-lactic acid) (PLLA)/PCL) nanofibrous 
scaffolds by adding epidermis-inducing factors such as epidermal growth factor (EGF) and 1,25-dihydroxyvitamin $\mathrm{D}_{3}$. The coating of collagen on scaffolds significantly increased the number of cells grown on this nanofibrous scaffold. BM-MSCs differentiated on collagen/PLLA/PCL nanofibrous scaffold showed a round keratinocyte morphology and expressed keratin 10, filaggrin, and involucrin protein, indicating the cell commitment to epidermal lineage [44]. Human BM-MSCs were grown on tissue culture plate coated with type I collagen gel. Depth-dependent differentiation of hMSCs to the epithelial lineage was observed with the thick collagen gel $(1900 \mu \mathrm{m})$, which generated significantly more cytokeratin 18 (CK18)-positive cells than collagen gel of a less thickness $(100 \mu \mathrm{m})$. The addition of all-trans retinoic acid (ATRA) enhanced CK18 expression and induced cluster formation in cells grown on the thick collagen gel. The authors argued the effect of gel depth on hMSC differentiation was caused by partial cytoskeletal disruption, suggesting type I collagen gel can be combined with ATRA to differentiate hMSCs to epithelial cells [45]. Corneal epithelial-like cells were generated from hair follicle- or skin fibroblast-derived hiPSCs. When coupling with BMP4, collagen IV promoted the differentiation of iPSCs to epithelial cells with a high purity [46].

Collagen substrates also support stem-cell differentiation to hepatocyte-like cells and MSCs. Synergistically with hepatocyte growth factor (HGF), collagen I promoted the hepatocyte generation of human adipose-derived stem cells in 2 weeks, as evidenced by upregulation of $\alpha$-fetoprotein, albumin, and $\alpha$-antitrypsin [47]. A one-step method using collagen I was reported to obtain MSC-like cells from hESCs and iPSCs, which could provide a readily available multipotent cell source for tissue engineering. Dissociated human H9 ESCs and HDFa-YK26 iPSCs were cultured on thin, fibrillar collagen I coatings with ROCK inhibitor Y27632. Interestingly, collagen I reduced stem-cell attachment compared to the culture plastic. After incubation for 10 days, colonies of spindle-shaped cells with MSC marker expression were obtained and identified. This study demonstrates that type I collagen can be used to guide a rapid and efficient derivation of MSC-like cells from human pluripotent stem cells (hPSCs) [48].

\subsubsection{Laminin}

As an important ECM protein, laminin has been reported to support the adhesion and functioning of a wide variety of stem cells [2, 49-52]. Of the different types of cell that can be differentiated on laminin, focus has been placed on neural cells. Laminin has been shown to promote NSC differentiation both in vitro and in vivo [50,51, 53-55]. The migration, expansion, and differentiation of human and mouse neural precursor cells (NPCs) into neurons and astrocytes were enhanced on laminin compared to fibronectin and Matrigel. The elongation of neurites from NPC-derived neurons was also observed on laminin. It was further revealed that the laminin-binding integrins $\alpha 3, \alpha 6, \alpha 7, \beta 1$, and $\beta 4$ expressed on these NPCs played a key role in laminin-dependent cell functions [51]. Again, the involvement of $\alpha 6 \beta 1$ integrin in laminin-induced neural progenitor expansion and neurite outgrowth was demonstrated $[54,56]$.

The direct incorporation of laminin in synthetic materials enhanced NSC attachment and functioning. Laminin-coated electrospun polyethersulfone fibers were shown to support the differentiation of rat NSCs to oligodendrocytes and neurons [57]. Laminin-1-coated methylcellulose scaffold promoted the survival and maturation of primary murine neurospheres with a significantly low level of apoptosis. The addition of laminin enhanced the 
expression of neuronal and oligodendrocyte precursor markers [56]. Poly- $D$-lysine (PDL) can be incorporated in laminin to provide a positively charged substrate and facilitate the differentiation of NSCs. For example, adult rat BM-MSCs were expanded on PDL- and laminin-1-coated glass in vitro, leading to the expression of nestin, a marker associated with neuronal progenitor cells, as compared to cells on TCPS- and PDL-coated surfaces, indicating laminin is crucial to NSC differentiation [58]. Similarly, PDL-laminin and Matrigel were reported to significantly promote the differentiation of hESC-derived EBs into neural progenitors, as well as to enhance neurite outgrowth compared to collagen, fibronectin, and PDL alone. Laminin was also found to stimulate the expansion and neurite outgrowth of hESC-derived neural progenitor cells in a dose-dependent manner [54].

Laminin-derived peptides can be conjugated to synthetic substrates and direct stemcell differentiation. A laminin-derived IKVAV motif was conjugated to a self-assembling peptide, RADA16, to make a peptide-based scaffold. The extended IKVAV sequence directed the adhesion and neural differentiation of NSCs encapsulated in the scaffold. Histological analysis following injection in rat brain revealed the RADA-IKVAV peptide enhanced survival of encapsulated NSCs and reduced the formation of glial astrocytes [50]. In another study, the IKVAV motif was conjugated to poly(2-hydroxyethyl methacrylate) (PHEMA) with Ac-CGGASIKVAVS-OH peptide. Porous Ac-CGGASIKVAVS-OHmodified PHEMA scaffolds significantly promoted the cell attachment, proliferation, and differentiation of human fetal NSCs [53]. However, modifying an interpenetrating polymer network (IPN) with laminin-derived IKVAV peptide (CSRARKQAASIKVAVSADR) failed to support the adhesion and differentiation of adult NSCs, suggesting other factors such as substrate stiffness and ligand density might jointly influence the overall performance of laminin-derived peptide in regulating NSC function [59].

Laminin-coated surfaces are able to support cardiogenesis and osteogenesis. Compared to collagen IV-coated scaffolds, laminin-coated 3D scaffolds promoted the development of $\mathrm{Flk}^{+}$cardiac progenitor cells from murine ESCs [41]. The late differentiation of cardiomyocytes from adipose-derived stem cells was enhanced on laminin, as demonstrated by a significant increase of sarcoendoplasmic reticulum calcium transport ATPase $2 \alpha$ (SERCA-2 $\alpha$ ) and myosin light chain $2 \alpha$ (MLC-2 $\alpha$ ) expression, as well as the number of desmin-positive cells after 5 weeks [60]. Laminin-modified poly(ethylene glycol) diacrylate (PEGDA) hydrogels increased the expression of osteogenic markers that might occur via the activation of $\alpha 2, \alpha v$, and $\alpha 6$ integrin subunits [49]. Moreover, laminin-induced osteogenesis was reported to be mediated by focal adhesion kinase (FAK) and extracellular signal-regulated kinases 1 and 2 (ERK1/2) pathways triggered by the interaction between integrins and laminin [61].

\subsubsection{Non-ECM-Derived Materials}

\subsubsection{Chitosan}

As a natural material obtained from chitin, a type of polysaccharide and a major component of crustacean exoskeleton, chitosan is biocompatible, biodegradable, and easy to process into various scaffold geometries for tissue engineering. Although chitosan has been shown to support the growth and differentiation of both pluripotent and multipotent stem cells, more attention has been focused on osteogenesis and chondrogenesis [62-72]. In bone and cartilage engineering, chitosan composites with other biocompatible materials, such 
as HA, calcium phosphate, and gelatin, are important substrates for osteogenic and chondrogenic differentiation. For example, a bilayer scaffold was developed for osteochondral tissue engineering. In this model, the chitosan/gelatin scaffold served as a chondrogenic layer and the HA/chitosan/gelatin complex was the osteogenic layer. MSCs seeded in these scaffolds exhibited enhanced proliferation and differentiation to chondrocytes and osteoblasts [63]. 3D chitosan/alginate porous scaffolds promoted CD105 ${ }^{+}$cell differentiation to chondral lineage, as evidenced by collagen II and SOX9 expression [68]. The 3D porous $\mathrm{HA} /$ chitosan/gelatin network was also proved to promote hMSC proliferation, retain progenicity, and improve osteogenic differentiation potential upon induction [71]. Notably, nanofibrous HA/chitosan scaffolds promoted the osteogenic commitment of murine MSCs in the absence of osteogenic supplementation, as demonstrated by the expression of osteoblast markers such as collagen I, Runx2, ALP, and osteocalcin [67]. Other than HA, calcium phosphate cement (CPC) has been used as a substrate with excellent osteoconductivity. Arginine-glycine-aspartic acid (RGD)-modified chitosan was incorporated with CPC to fabricate a CPC/chitosan/RGD scaffold. hESC-derived MSCs on this scaffold underwent osteogenesis with enhanced marker expression and mineral deposition [64]. The CPC/chitosan composite also supported human UC-MSC differentiation to osteoblasts with higher mineral synthesis than BM-MSCs [69].

The topography of chitosan-based scaffolds influences stem-cell behavior. It has been found that less hESCs adhere to chitosan fibers than to membranes but that chitosan fibers promote topography-mediated ESC differentiation compared to membranes. Moreover, the fiber size is important to stem-cell fate. hESCs underwent ectoderm differentiation when the fiber diameter was $400 \mathrm{~nm}$ and $1.1 \mu \mathrm{m}$; in contrast, the cells were directed to endodermal and mesodermal lineages with smaller diameter fibers $(200 \mathrm{~nm})$ [65].

\subsection{Synthetic Biomaterials}

The biomimetic property of natural biomaterials, especially their capability for presenting ligands to initiate intracellular pathways and direct subsequent stem-cell differentiation, is unavailable to many synthetic materials. However, the advantages of synthetic materials over natural biomaterials include the tunability of material properties such as hydrophobicity, mechanical strength, porosity, and topography. More importantly, synthetic materials are capable of delivering drugs and growth factors in a controlled manner. Synthetic biomaterials are preferentially biodegradable and bioresorbable, with nontoxic degradation products that can be readily removed from the body with new tissue formation. According to this notion, this section will focus on polyesters and poly(ethylene glycol) (PEG), which are highly biocompatible synthetic polymers with tunable properties that can be adapted for tissue engineering and regenerative medicine. Recent studies using selected polymers to differentiate stem cells are summarized in Table 11.1.

\subsubsection{Polyesters}

\subsubsection{Poly(Lactic Acid) and Poly(Glycolic Acid) Copolymers}

Polyesters have been extensively used in tissue engineering and regenerative medicine, as their biocompatibility and biodegradability have been well demonstrated. They undergo hydrolysis and enzyme-mediated degradation both in vitro and in vivo. The polyesters have 


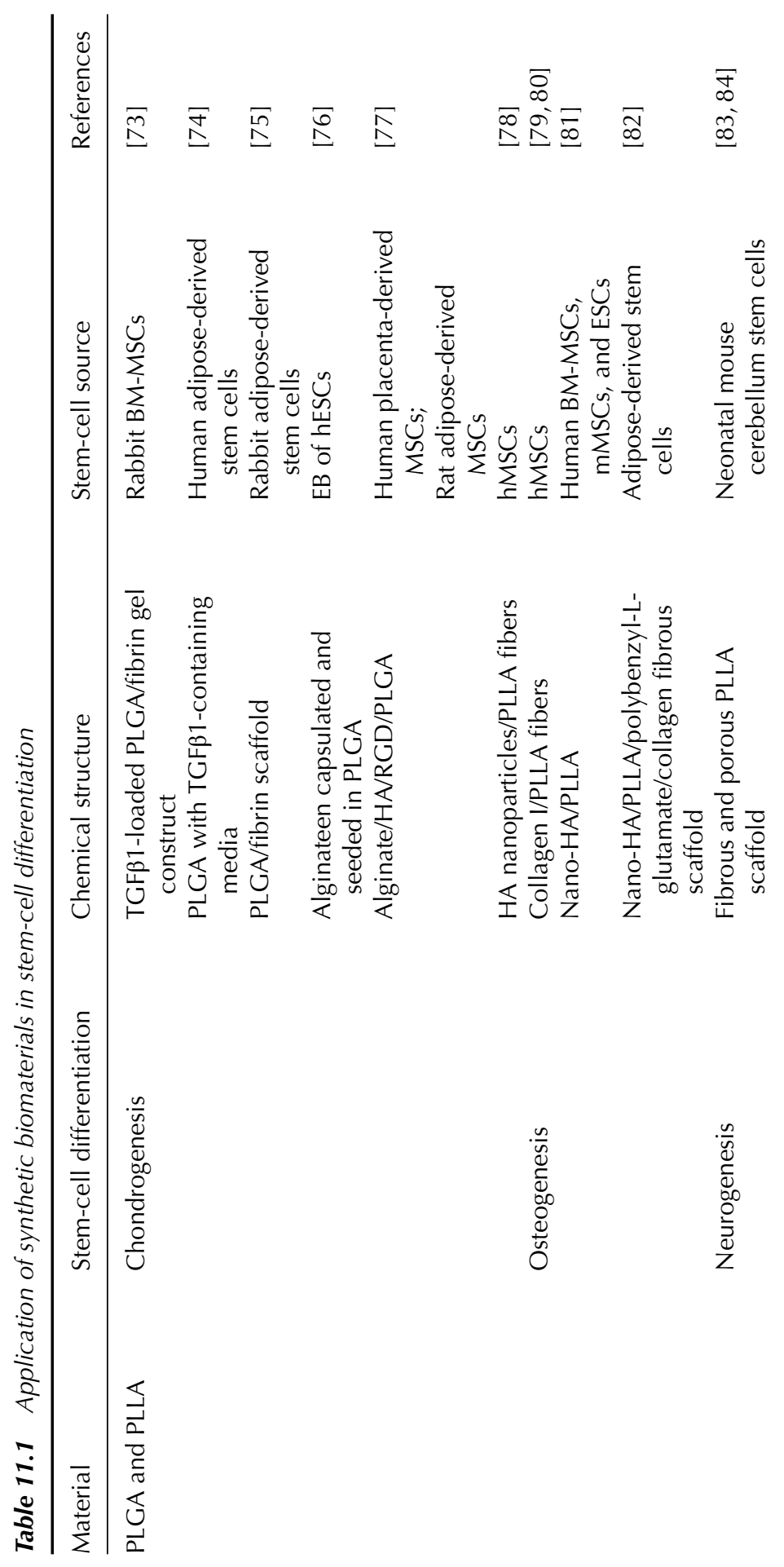




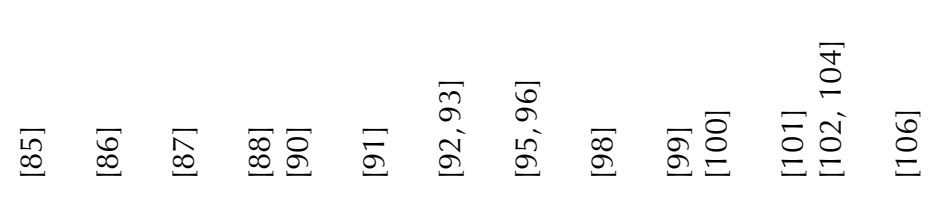

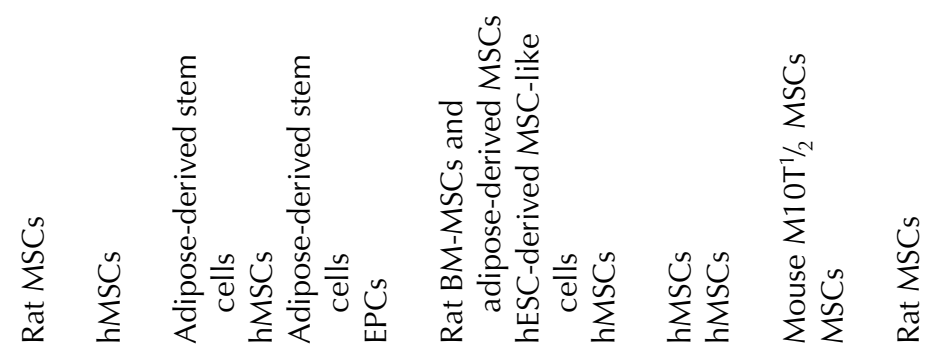

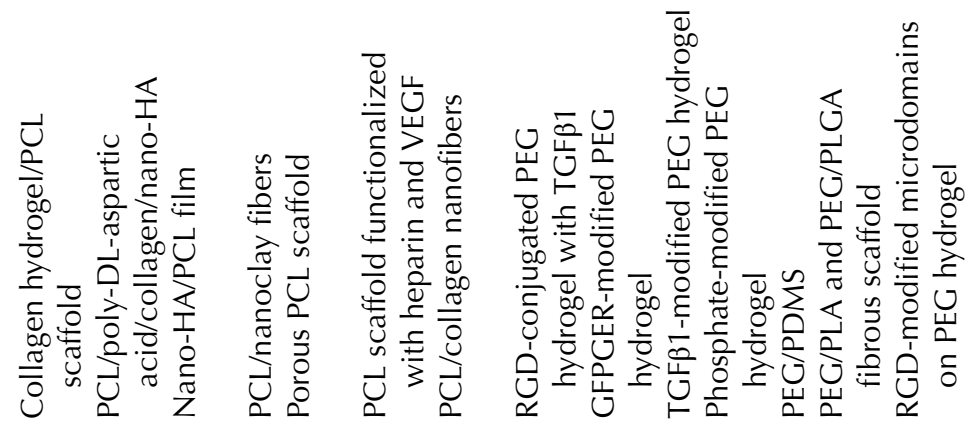

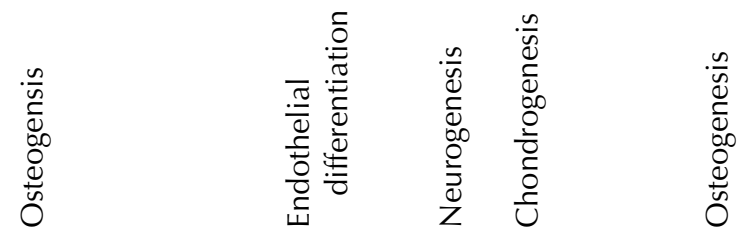

$\varpi$

戒 
good processability and can be fabricated into multiple platforms, such as thin films, porous scaffolds, particles, and micro- and nanofibers, showing excellent versatility for stem-cell therapy. Poly(lactic acid) (PLA), poly(glycolic acid) (PGA), and their copolymers PLLA and PLGA are the well-known representatives that have been approved in clinical uses for a few decades.

The PLLA and PLGA copolymers have been used in chondrogenesis and osteogenesis due to the requirement for high material modulus in regeneration of these tissues. However, the biological inertness of the polymer scaffolds limits cell-material interaction. Therefore, natural materials are often incorporated to enhance cell retention and reaction in these scaffolds. For example, fibrin gel was added to PLGA scaffolds to support the differentiation of rat BM-MSC and human adipose-derived stem cells to chondrocytes under TGF $\beta 1$ treatment [73-75]. Stem cells can also be encapsulated in alginate before being loaded to PLGA scaffolds. Human ESC-derived EBs were suspended in alginate and seeded into PLGA 3D scaffolds. After implantation, embedded cells expressed chondrocyte markers and formed cartilage-like tissue in mice [76]. The addition of calcium-deficient HA and RGD to alginate gel enhanced the chondrogenesis of both adipose- and placenta-derived MSCs [77]. The chondrogenic differentiation of hMSCs was promoted by HA nanoparticleloaded electrospun PLLA fibers [78].

Porous or fibrous PLGA and PLLA scaffolds have been used in bone generation; for example, the electrospun PLLA/collagen nanofibers promoted osteogenic differentiation compared to PLLA scaffolds without collagen [79]. After implantation in rat calvarial defects, the PLLA/collagen nanofibrous scaffolds improved the migration and infiltration of more osteocalcin- and Smad5-positive cells than an empty defect control [80]. The composites of PLLA nanofibrous mats blended with nano-HA promoted osteogenic differentiation of human BM-MSCs, murine MSCs, and ESCs [81]. Adipose-derived MSCs can also be directed to osteoblasts on nano-HA-enriched PLLA/poly-benzyl-L-glutamate/collagen nanofibrous scaffolds [82].

The fibrous PLLA and PLGA scaffolds are able to support stem-cell differentiation to other lineages, including neural cells. It was reported that porous and fibrous PLLA scaffolds induced neurite outgrowth from neonatal mouse cerebellum stem cells [83]. Furthermore, the NSC behavior was controlled by topographical features such as fiber size and alignment. Aligned PLLA fibers with a diameter of $500 \mathrm{~nm}$ led to higher cell viability and proliferation compared to either smaller or bigger fibers, as opposed to unaligned fibers. Importantly, the fiber size and the alignment influenced cell differentiation as well. Cell outgrowth was higher on $500 \mathrm{~nm}$ aligned fibers than on $307 \mathrm{~nm}$ fibers, but this was totally opposite to unaligned fibers, indicating the importance of topographical features in NSC differentiation [84].

\subsubsection{Poly(ع-Caprolactone)}

PCL is another commonly used polyester in tissue engineering. One of the advantages of PCL over PLGA and PLLA is the generation of nonacidic degradation products; thus, it usually induces less inflammatory responses than PLGA or PLLA. PCL is more hydrophobic and more bio-inert than PLGA and PLLA, so additional treatments or surface modifications are often required to make it biomimetic and thus support stem-cell functions. The 
capability of PCL to direct stem-cell commitment has been documented, including osteogenesis and chondrogenesis. PCL has been frequently used in bone engineering, with the incorporation of other materials to enhance its strength and improve cell-material interaction. For example, rat MSCs were embedded in collagen hydrogels before being cast into macro-channeled PCL scaffolds to increase cell retention and proliferation. Osteoblast marker expression and mineralization were significantly enhanced under flow perfusion, which induced shear stress to stem cells and upregulated c-Fos and COX-2 expression, indicating a force-driven differentiation of stem cells in this PCL scaffold [85]. A hybrid nanofibrous scaffold made of PCL/poly $D, L$-aspartic acid/collagen/nano-HA was applied to differentiate hMSCs to osteoblasts [86]. The shape of the nano-HA in PCL scaffolds is crucial to osteogenesis; for example, rod-shaped HA nanoparticles induce more osteogenic marker expression in adipose-derived MSCs than do spherical HA nanoparticles [87]. Halloysite nanoclay, an inorganic filler material, was used to fabricate PCL/nanoclay fibrous scaffolds that were mechanically superior to PCL scaffolds alone. Increased ALP activity was observed in hMSCs grown on this scaffold, showing the PCL/nanoclay composite directed MSCs to osteoblast lineage [88]. The ability to mimic ECM structures makes nanoand microfibrous scaffolds very attractive. However, for synthetic polymers such as PLLA, PLGA, and PCL, which degrade slowly, the small pore size of these fibrous scaffolds often hinders cell filtration and tissue ingrowth, which is critical in tissue engineering. Thus, in a previous study, water-soluble PEG fibers ("sacrificial fibers") were blended to make PCL/collagen/nano-HA fibers. The PEG fibers were then removed by soaking the hybrid scaffold in water, leaving PCL scaffold with larger pores, which facilitated MSC infiltration into the scaffold. This method therefore provides an easy way to fabricate porous fibrous scaffolds that can facilitate stem-cell migration [89].

Commitment of stem cells to other lineages on PCL scaffolds has been reported. Adiposederived stem cells were differentiated to endothelial cells on porous PCL scaffolds, with the supplement of endothelial differentiation medium [90]. EPCs were seeded in porous PCL scaffolds functionalized with heparin and VEGF. Seven days after subcutaneous implantation in non-obese diabetic/severe combined immunodeficiency (NOD-SCID) mice, scaffolds loaded with EPCs promoted angiogenesis and vasculogenesis with CD31 ${ }^{+}$vessel formation [91]. The topography of PCL fibrous scaffolds again plays a crucial role in stemcell differentiation, especially in neurogenesis. Aligned or randomly oriented PCL and PCL/collagen nanofibers were prepared to investigate the topographical effect on growth and neural differentiation of rat BM-MSCs and adipose-derived MSCs. The differentiation to oligodendrocytes was more pronounced on aligned PCL/collagen fibers than was differentiation to other neural cell types [92]. In another study, aligned PCL nanofibers significantly upregulated the expression of neural markers in MSCs compared to cells on tissue culture plastic. The combination of sustained release of retinoic acid (RA) with aligned PCL nanofibers significantly changed cell morphology and enhanced neural marker expression, especially expression of the mature neural marker microtube-associated protein 2 (MAP2). Positive staining of synaptophysin occurred only in cells on RA-releasing aligned scaffolds. These results highlight the regulatory effect of the topography of drug-eluting PCL nanofibers on neural differentiation [93]. Biofunctionalization of PCL scaffolds provides another way to efficiently promote the neural differentiation of stem cells. For example, surface modification of PCL fibers with neurotrophin enhanced NSC differentiation to oligodendrocytes [94]. 


\subsubsection{Polyethylene Glycol}

PEG is a biocompatible, nondegradable hydrophilic polymer. It is an extremely versatile material and has been extensively used in synthesizing polymeric biomaterials for different purposes. PEGDA can be easily crosslinked when exposed to UV light with a photoinitiator to form hydrogels that can encapsulate stem cells. However, since PEG is so hydrophilic, it markedly repels the adsorption of proteins, which makes it non-cell-adhesive. Therefore, modifications to PEG hydrogels with bioactive ligands have been performed to enhance cell-material interactions, making PEG an ideal blank material with which to study the functions of different ligands. For example, the cell-adhesive peptide RGD was conjugated to PEGDA hydrogels to encapsulate and support the differentiation of hESC-derived MSC-like cells to chondrocytes [95]. TGF $\beta$-supplemented, RGD-modified PEGDA hydrogels synergistically directed the chondrogenic differentiation of hESCs [96]. Moreover, the RGD concentration required to promote rat MSC adhesion on PEGDA hydrogel was reported to be within $0.107-0.143 \mathrm{mM}$ [97]. In another study, a collagen mimetic peptide containing a GFPGER sequence was conjugated to PEG hydrogels, which provided an extracellular environment similar to collagen and promoted chondrogenic differentiation of hMSCs [98]. Other than adhesive ligands, PEG hydrogels can be covalently modified with growth factors or bioactive ligands to guide stem-cell differentiation. TGF $\beta$ has been covalently tethered to PEGDA hydrogels by photoinitiated thiol-acrylate polymerization. hMSCs were encapsulated in PEG hydrogels and the tethered TGF $\beta$ promoted chondrogenesis after 3 weeks, with increased expression of collagen II and glycosaminoglycan [99]. Phosphate (PO(4))-functionalized PEG hydrogels increased the adsorption of serum proteins compared to the nonfunctionalized PEG hydrogel, which subsequently improved attachment and spreading of hMSCs. The improvement of osteognesis by phosphate was further demonstrated via activation of $\beta 1 / \beta 3$ integrins and the FAK pathway [100].

PEG can be modified with other natural and synthetic polymers to improve its hydrophobicity and/or elasticity in order to support stem-cell attachment and differentiation. For example, the hydrophobicity of PEG can be changed by adding poly(dimethylsiloxane) (PDMS), which elevated osteogenic differentiation of mouse $10 \mathrm{~T}^{1} / 2$ MSCs as the PDMS : PEG ratio increased [101]. Hybrid PEG/PLA fibrous scaffolds with interconnected pores favored growth of MSCs and enhanced their differentiation to osteoblast-like cells [102]. A photopolymerized PEG hydrogel with poly(L-lysine) increased the survival and differentiation of NPCs. About 55\% of the cells cultured in gels differentiated into a mature neural cell type [103]. Acrylate-N-hydroxysuccinimide PEG was applied as a spacer in order to covalently tether BMP2 to a PLGA scaffold. The PEG/BMP2/PLGA scaffold improved de novo bone formation in animals by promoting osteogenesis of seeded MSCs [104]. Mouse $10 \mathrm{~T}^{1} / 2$ MSCs were encapsulated in fibrinogen, fibronectin, or laminin-1 conjugated PEGDA hydrogels and the osteogenic differentiation in these hydrogels was studied. Cells in fibrinogen and laminin-modified PEG hydrogels increased osteoblast marker expression compared to cells in fibronectin-modified hydrogels, due to altered integrin profiles in the differentiation process [49].

Instead of modifying PEG scaffolds to be more cell-friendly, the hydrophilicity of PEG provides a useful tool by which to repel cell attachment and change substrate topographical properties, which influences the stem-cell morphological features and directs stem-cell differentiation. For example, micropatterning PEG hydrogel on to a cell-adhesive substrate 
will isolate cell-adhesive and nonadhesive areas (e.g. forming microwells). By changing the size and shape of the adhesive domains surrounded by PEG, stem-cell differentiation within them can be controlled. Moreover, bioactive ligands in cell-adhesive domains can be varied to assay stem-cell differentiation under different conditions, providing a highthroughput method of screening hundreds of immobilized or soluble ligands simultaneously [105]. For example, RGD-modified microdomains were patterned on PEG hydrogels and the differentiation of rat MSCs in RGD-modified domains was studied. It was found that both osteogenic and adipogenic differentiation were promoted by cell-cell contact, whereas isolated individual cells showed less differentiation than aggregated cells [106]. PEG/PLA line-patterned substrate reduced multiple extension of neurites and stimulated the bidirectional neurite budding of PC12 cells [107]. In contrast to the concept of confining cells in adhesive microwells, the protein- and cell-repellant nature of PEG can be used to control the size, shape, and homogeneity of pluripotent stem cell-derived EBs in nonadhesive microdomains. Micropatterning of PEGDA hydrogels on glass produced PEG microwells with a diameter of $50 \mu \mathrm{m}$, which facilitated the homogeneous murine ESCderived EB formation on a microarray chip, enabling the high-throughput manipulation of ESCs [108]. Sakai et al. compared the PEG-modified microwells and micropatterned chip with gelatin spots on a PEG-modified surface in EB proliferation and differentiation. They found EBs in the microwells were more likely to retain the pluripotency, whereas the gelatin-micropatterned chip promoted EB growth and differentiation to both endodermal and mesodermal lineages [109]. The size of EB played a role in directing stem-cell differentiation. Size-controlled EBs were produced by microwell array and were encapsulated in PEG-4-arm acrylate hydrogels with and without RGD conjugation. The size of EBs increased when cultured on unmodified PEG hydrogels, and such cultured EBs showed enhanced cardiogenic differentiation compared to RGD-PEG hydrogels. The differentiation to endothelial cells was also dependent on the size of EBs, but it was accelerated in RGD-PEG hydrogels within 3-5 days. Thus, PEG hydrogel may induce cardiogenic differentiation and the RGD sequence could be a driving force of direct differentiation to endothelial cells [110].

\subsection{Conclusion}

Biomaterials provide a unique platform from which to direct stem-cell differentiation synergistically with soluble factors. Naturally derived biomaterials are more closely mimetic to the stem cell niche in vivo than synthetic materials as they can provide more functional ligands to regulate stem-cell adhesion, proliferation, and differentiation. However, their mechanical properties, degradability, and hydrophobicity are less tunable to the requirements of different applications than those of synthetic biomaterials; synthetic materials, especially polymers, are capable of tuning their properties for ligand functionalization, adjustable degradation, surface patterning, and controlled release of therapeutics. Thus, in the development of tissue-engineering scaffolds, natural materials (or short motifs derived from these materials) and synthetic materials are often combined to achieve optimal cell functions. Stem cells, as a reliable cell source, emerge as a powerful tool for tissue engineering and regenerative medicine. There is no doubt that the combination of stem cells 
with biomaterials will lead to huge breakthroughs in this area. Development of biomaterials that direct stem-cell differentiation to specific cell lineages, despite the exciting findings reviewed in this chapter, remains immature. Thus, further in-depth studies are required to allow us to understand the mechanisms behind the regulation of stem-cell fate by biomaterials.

\section{References}

(1) Kleinman, H.K., McGarvey, M.L., Liotta, L.A. et al. (1982) Isolation and characterization of type IV procollagen, laminin, and heparan sulfate proteoglycan from the EHS sarcoma. Biochemistry, 21 (24), 6188-6193.

(2) Lam, M.T. and Longaker, M.T. (2012) Comparison of several attachment methods for human iPS, embryonic and adipose-derived stem cells for tissue engineering. Journal of Tissue Engineering and Regenerative Medicine, 6 (Suppl 3), s80-s86.

(3) Xu, X., Browning, V.L., and Odorico, J.S. (2011) Activin, BMP and FGF pathways cooperate to promote endoderm and pancreatic lineage cell differentiation from human embryonic stem cells. Mechanisms of Development, 128 (7-10), 412-427.

(4) Rufaihah, A.J., Huang, N.F., Jame, S. et al. (2011) Endothelial cells derived from human iPSCS increase capillary density and improve perfusion in a mouse model of peripheral arterial disease. Arteriosclerosis, Thrombosis, and Vascular Biology, 31 (11), e72-e79.

(5) Liu, Y., Wang, X., Kaufman, D.S., and Shen, W. (2011) A synthetic substrate to support early mesodermal differentiation of human embryonic stem cells. Biomaterials, 32 (32), 8058-8066.

(6) Lin, S. and Talbot, P. (2011) Methods for culturing mouse and human embryonic stem cells. Methods in Molecular Biology, 690, 31-56.

(7) Tsai, Z.Y., Singh, S., Yu, S.L. et al. (2010) A feeder-free culture using autogeneic conditioned medium for undifferentiated growth of human embryonic stem cells: comparative expression profiles of mRNAs, microRNAs and proteins among different feeders and conditioned media. BMC Cell Biology [Electronic Resource], 11, 76.

(8) Banerjee, I., Sharma, N., and Yarmush, M. (2011) Impact of co-culture on pancreatic differentiation of embryonic stem cells. Journal of Tissue Engineering and Regenerative Medicine, 5 (4), 313-323.

(9) Kane, N.M., Meloni, M., Spencer, H.L. et al. (2010) Derivation of endothelial cells from human embryonic stem cells by directed differentiation: analysis of microRNA and angiogenesis in vitro and in vivo. Arteriosclerosis, Thrombosis, and Vascular Biology, 30 (7), 1389-1397.

(10) Liu, T., Zhang, S., Chen, X. et al. (2010) Hepatic differentiation of mouse embryonic stem cells in three-dimensional polymer scaffolds. Tissue Engineering Part A, 16 (4), 1115-1122.

(11) Chaturvedi, G., Simone, P.D., Ain, R. et al. (2009) Noggin maintains pluripotency of human embryonic stem cells grown on Matrigel. Cell Proliferation, 42 (4), 425-433.

(12) Ishii, T., Fukumitsu, K., Yasuchika, K. et al. (2008) Effects of extracellular matrixes and growth factors on the hepatic differentiation of human embryonic stem cells. American Journal of Physiology - Gastrointestinal and Liver Physiology, 295 (2), G313-G321.

(13) Gong, J., Sagiv, O., Cai, H. et al. (2008) Effects of extracellular matrix and neighboring cells on induction of human embryonic stem cells into retinal or retinal pigment epithelial progenitors. Experimental Eye Research, 86 (6), 957-965.

(14) Boyd, N.L., Dhara, S.K., Rekaya, R. et al. (2007) BMP4 promotes formation of primitive vascular networks in human embryonic stem cell-derived embryoid bodies. Experimental Biology and Medicine (Maywood, N.J.), 232 (6), 833-843.

(15) Rajesh, D., Chinnasamy, N., Mitalipov, S.M. et al. (2007) Differential requirements for hematopoietic commitment between human and rhesus embryonic stem cells. Stem Cells, 25 (2), 490-499.

(16) Nakagami, H., Nakagawa, N., Takeya, Y. et al. (2006) Model of vasculogenesis from embryonic stem cells for vascular research and regenerative medicine. Hypertension, 48 (1), 112-119. 
(17) Yao, S., Chen, S., Clark, J. et al. (2006) Long-term self-renewal and directed differentiation of human embryonic stem cells in chemically defined conditions. Proceedings of the National Academy of Sciences of the United States of America, 103 (18), 6907-6912.

(18) Arufe, M.C., Lu, M., Kubo, A. et al. (2006) Directed differentiation of mouse embryonic stem cells into thyroid follicular cells. Endocrinology, 147 (6), 3007-3015.

(19) Zhang, J., Klos, M., Wilson, G.F. et al. (2012) Extracellular matrix promotes highly efficient cardiac differentiation of human pluripotent stem cells: the matrix sandwich method. Circulation Research, 111 (9), 1125-1136.

(20) Uemura, M., Refaat, M.M., Shinoyama, M. et al. (2010) Matrigel supports survival and neuronal differentiation of grafted embryonic stem cell-derived neural precursor cells. J. Neurosci. Res., 88 (3), 542-551.

(21) Trivedi, P. and Hematti, P. (2008) Derivation and immunological characterization of mesenchymal stromal cells from human embryonic stem cells. Experimental Hematology, 36 (3), 350-359.

(22) Barsotti, M.C., Magera, A., Armani, C. et al. (2011) Fibrin acts as biomimetic niche inducing both differentiation and stem cell marker expression of early human endothelial progenitor cells. Cell Proliferation, 44 (1), 33-48.

(23) Sreerekha, P.R., Divya, P., and Krishnan, L.K. (2006) Adult stem cell homing and differentiation in vitro on composite fibrin matrix. Cell Proliferation, 39 (4), 301-312.

(24) Catelas, I., Sese, N., Wu, B.M. et al. (2006) Human mesenchymal stem cell proliferation and osteogenic differentiation in fibrin gels in vitro. Tissue Engineering, 12 (8), 23852396.

(25) Liao, H.T., Chen, C.T., Chen, C.H. et al. (2011) Combination of guided osteogenesis with autologous platelet-rich fibrin glue and mesenchymal stem cell for mandibular reconstruction. Journal of Trauma, 70 (1), 228-237.

(26) Park, K.H., Kim, H., Moon, S., and Na, K. (2009) Bone morphogenic protein-2 (BMP-2) loaded nanoparticles mixed with human mesenchymal stem cell in fibrin hydrogel for bone tissue engineering. Journal of Bioscience and Bioengineering, 108 (6), 530-537.

(27) de la Puente, P., Ludena, D., Lopez, M. et al. (2013) Differentiation within autologous fibrin scaffolds of porcine dermal cells with the mesenchymal stem cell phenotype. Experimental Cell Research, 319 (3), 144-152.

(28) Proulx, M.K., Carey, S.P., Ditroia, L.M. et al. (2011) Fibrin microthreads support mesenchymal stem cell growth while maintaining differentiation potential. Journal of Biomedical Materials Research. Part A, 96 (2), 301-312.

(29) Lee, Y.B., Polio, S., Lee, W. et al. (2010) Bio-printing of collagen and VEGF-releasing fibrin gel scaffolds for neural stem cell culture. Experimental Neurology, 223 (2), 645-652.

(30) Willerth, S.M., Rader, A., and Sakiyama-Elbert, S.E. (2008) The effect of controlled growth factor delivery on embryonic stem cell differentiation inside fibrin scaffolds. Stem Cell Research, 1 (3), 205-218.

(31) Antoon, R., Yeger, H., Loai, Y. et al. (2012) Impact of bladder-derived acellular matrix, growth factors, and extracellular matrix constituents on the survival and multipotency of marrow-derived mesenchymal stem cells. Journal of Biomedical Materials Research. Part A, 100 (1), 72-83.

(32) Huang, Y., Lin, L., Yu, X. et al. (2013) Functional involvements of heterogeneous nuclear ribonucleoprotein A1 in smooth muscle differentiation from stem cells in vitro and in vivo. Stem Cells, 31 (5), 906-917.

(33) Haghighipour, N., Heidarian, S., Shokrgozar, M.A., and Amirizadeh, N. (2012) Differential effects of cyclic uniaxial stretch on human mesenchymal stem cell into skeletal muscle cell. Cell Biology International, 36 (7), 669-675.

(34) Yu, H., Lui, Y.S., Xiong, S. et al. (2013) Insights into the role of focal adhesion modulation in myogenic differentiation of human mesenchymal stem cells. Stem Cells and Development, 22 (1), 136-147.

(35) Chen, X., Zhang, F., He, X. et al. (2013) Chondrogenic differentiation of umbilical cordderived mesenchymal stem cells in type I collagen-hydrogel for cartilage engineering. Injury, 44 (4), 540-549. 
(36) Fong, C.Y., Subramanian, A., Gauthaman, K. et al. (2012) Human umbilical cord Wharton's jelly stem cells undergo enhanced chondrogenic differentiation when grown on nanofibrous scaffolds and in a sequential two-stage culture medium environment. Stem Cell Reviews, 8 (1), 195-209.

(37) Raabe, O., Shell, K., Fietz, D. et al. (2013) Tenogenic differentiation of equine adipose-tissuederived stem cells under the influence of tensile strain, growth differentiation factors and various oxygen tensions. Cell and Tissue Research, 352 (3), 509-521.

(38) Murphy, C.M., Matsiko, A., Haugh, M.G. et al. (2012) Mesenchymal stem cell fate is regulated by the composition and mechanical properties of collagen-glycosaminoglycan scaffolds. Journal of the Mechanical Behavior of Biomedical Materials, 11, 53-62.

(39) Naito, H., Dohi, Y., Zimmermann, W.H. et al. (2011) The effect of mesenchymal stem cell osteoblastic differentiation on the mechanical properties of engineered bone-like tissue. Tissue Engineering Part A, 17 (17-18), 2321-2329.

(40) Naito, H., Yoshimura, M., Mizuno, T. et al. (2013) The advantages of three-dimensional culture in a collagen hydrogel for stem cell differentiation. Journal of Biomedical Materials Research Part A, 101 (10), 2838-2846.

(41) Heydarkhan-Hagvall, S., Gluck, J.M., Delman, C. et al. (2012) The effect of vitronectin on the differentiation of embryonic stem cells in a 3D culture system. Biomaterials, 33 (7), 2032-2040.

(42) Horton, R.E. and Auguste, D.T. (2012) Synergistic effects of hypoxia and extracellular matrix cues in cardiomyogenesis. Biomaterials, 33 (27), 6313-6319.

(43) Zeng, D., Ou, D.B., Wei, T. et al. (2013) Collagen/beta(1) integrin interaction is required for embryoid body formation during cardiogenesis from murine induced pluripotent stem cells. BMC Cell Biology [Electronic Resource], 14, 5.

(44) Jin, G., Prabhakaran, M.P., and Ramakrishna, S. (2011) Stem cell differentiation to epidermal lineages on electrospun nanofibrous substrates for skin tissue engineering. Acta Biomaterialia, 7 (8), 3113-3122.

(45) Takebayashi, T., Horii, T., Denno, H. et al. (2013) Human mesenchymal stem cells differentiate to epithelial cells when cultured on thick collagen gel. Bio-Medical Materials and Engineering, 23 (1), 143-153.

(46) Shalom-Feuerstein, R., Serror, L., De La Forest Divonne, S. et al. (2012) Pluripotent stem cell model reveals essential roles for miR-450b-5p and miR-184 in embryonic corneal lineage specification. Stem Cells, 30 (5), 898-909.

(47) Ghaedi, M., Tuleuova, N., Zern, M.A. et al. (2011) Bottom-up signaling from HGF-containing surfaces promotes hepatic differentiation of mesenchymal stem cells. Biochemical and Biophysical Research Communications, 407 (2), 295-300.

(48) Liu, Y., Goldberg, A.J., Dennis, J.E. et al. (2012) One-step derivation of mesenchymal stem cell (MSC)-like cells from human pluripotent stem cells on a fibrillar collagen coating. Plos One, 7 (3), e33225.

(49) Becerra-Bayona, S., Guiza-Arguello, V., Qu, X. et al. (2012) Influence of select extracellular matrix proteins on mesenchymal stem cell osteogenic commitment in three-dimensional contexts. Acta Biomaterialia, 8 (12), 4397-404.

(50) Cheng, T.Y., Chen, M.H., Chang, W.H. et al. (2013) Neural stem cells encapsulated in a functionalized self-assembling peptide hydrogel for brain tissue engineering. Biomaterials, 34 (8), 2005-2016.

(51) Flanagan, L.A., Rebaza, L.M., Derzic, S. et al. (2006) Regulation of human neural precursor cells by laminin and integrins. J. Neurosci. Res., 83 (5), 845-856.

(52) Heng, B.C., Li, J., Chen, A.K. et al. (2012) Translating human embryonic stem cells from 2dimensional to 3-dimensional cultures in a defined medium on laminin- and vitronectin-coated surfaces. Stem Cells and Development, 21 (10), 1701-1715.

(53) Kubinova, S., Horak, D., Kozubenko, N. et al. (2010) The use of superporous AcCGGASIKVAVS-OH-modified PHEMA scaffolds to promote cell adhesion and the differentiation of human fetal neural precursors. Biomaterials, 31 (23), 5966-5975.

(54) Ma, W., Tavakoli, T., Derby, E. et al. (2008) Cell-extracellular matrix interactions regulate neural differentiation of human embryonic stem cells. BMC Developmental Biology [Electronic Resource], 8, 90. 
(55) Tate, C.C., Shear, D.A., Tate, M.C. et al. (2009) Laminin and fibronectin scaffolds enhance neural stem cell transplantation into the injured brain. Journal of Tissue Engineering and Regenerative Medicine, 3 (3), 208-217.

(56) Stabenfeldt, S.E., Munglani, G., Garcia, A.J., and LaPlaca, M.C. (2010) Biomimetic microenvironment modulates neural stem cell survival, migration, and differentiation. Tissue Engineering Part A, 16 (12), 3747-3758.

(57) Christopherson, G.T., Song, H., and Mao, H.Q. (2009) The influence of fiber diameter of electrospun substrates on neural stem cell differentiation and proliferation. Biomaterials, 30 (4), 556-564.

(58) Ho, M., Yu, D., Davidsion, M.C., and Silva, G.A. (2006) Comparison of standard surface chemistries for culturing mesenchymal stem cells prior to neural differentiation. Biomaterials, 27 (24), 4333-4339.

(59) Saha, K., Irwin, E.F., Kozhukh, J. et al. (2007) Biomimetic interfacial interpenetrating polymer networks control neural stem cell behavior. Journal of Biomedical Materials Research. Part A, 81 (1), 240-249.

(60) van Dijk, A., Niessen, H.W., Zandieh Doulabi, B. et al. (2008) Differentiation of human adipose-derived stem cells towards cardiomyocytes is facilitated by laminin. Cell and Tissue Research, 334 (3), 457-467.

(61) Salasznyk, R.M., Klees, R.F., Boskey, A., and Plopper, G.E. (2007) Activation of FAK is necessary for the osteogenic differentiation of human mesenchymal stem cells on laminin-5. Journal of Cellular Biochemistry, 100 (2), 499-514.

(62) Budiraharjo, R., Neoh, K.G., and Kang, E.T. (2012) Hydroxyapatite-coated carboxymethyl chitosan scaffolds for promoting osteoblast and stem cell differentiation. Journal of Colloid and Interface Science, 366 (1), 224-232.

(63) Chen, J., Chen, H., Li, P. et al. (2011) Simultaneous regeneration of articular cartilage and subchondral bone in vivo using MSCs induced by a spatially controlled gene delivery system in bilayered integrated scaffolds. Biomaterials, 32 (21), 4793-805.

(64) Chen, W., Zhou, H., Weir, M.D. et al. (2013) Human embryonic stem cell-derived mesenchymal stem cell seeding on calcium phosphate cement-chitosan-RGD scaffold for bone repair. Tissue Engineering Part A, 19(7-8), 915-927.

(65) Cooper, A., Leung, M., and Zhang, M. (2012) Polymeric fibrous matrices for substratemediated human embryonic stem cell lineage differentiation. Macromolecular Bioscience, 12 (7), 882-892.

(66) Costa-Pinto, A.R., Salgado, A.J., Correlo, V.M. et al. (2008) Adhesion, proliferation, and osteogenic differentiation of a mouse mesenchymal stem cell line (BMC9) seeded on novel melt-based chitosan/polyester 3D porous scaffolds. Tissue Engineering Part A, 14 (6), 10491057.

(67) Peng, H., Yin, Z., Liu, H. et al. (2012) Electrospun biomimetic scaffold of hydroxyapatite/chitosan supports enhanced osteogenic differentiation of mMSCs. Nanotechnology, 23 (48), 485102.

(68) Qi, J., Chen, A., You, H. et al. (2011) Proliferation and chondrogenic differentiation of CD105positive enriched rat synovium-derived mesenchymal stem cells in three-dimensional porous scaffolds. Biomedical Materials, 6 (1), 015006.

(69) Xu, H.H., Zhao, L., and Weir, M.D. (2010) Stem cell-calcium phosphate constructs for bone engineering. Journal of Dental Research, 89 (12), 1482-1488.

(70) Zahir, T., Nomura, H., Guo, X.D. et al. (2008) Bioengineering neural stem/progenitor cellcoated tubes for spinal cord injury repair. Cell Transplantation, 17 (3), 245-254.

(71) Zhao, F., Grayson, W.L., Ma, T. et al. (2006) Effects of hydroxyapatite in 3-D chitosan-gelatin polymer network on human mesenchymal stem cell construct development. Biomaterials, 27 (9), 1859-1867.

(72) Zhao, L., Weir, M.D., and Xu, H.H. (2010) Human umbilical cord stem cell encapsulation in calcium phosphate scaffolds for bone engineering. Biomaterials, 31 (14), 38483857.

(73) Wang, W., Li, B., Yang, J. et al. (2010) The restoration of full-thickness cartilage defects with BMSCs and TGF-beta 1 loaded PLGA/fibrin gel constructs. Biomaterials, 31 (34), 8964 8973. 
(74) Mehlhorn, A.T., Zwingmann, J., Finkenzeller, G. et al. (2009) Chondrogenesis of adiposederived adult stem cells in a poly-lactide-co-glycolide scaffold. Tissue Engineering Part A, 15 (5), 1159-1167.

(75) Wei, Y., Hu, H., Wang, H. et al. (2009) Cartilage regeneration of adipose-derived stem cells in a hybrid scaffold from fibrin-modified PLGA. Cell Transplantation, 18 (2), 159-170.

(76) Bai, H.Y., Chen, G.A., Mao, G.H. et al. (2010) Three step derivation of cartilage like tissue from human embryonic stem cells by 2D-3D sequential culture in vitro and further implantation in vivo on alginate/PLGA scaffolds. Journal of Biomedical Materials Research. Part A, 94 (2), 539-546.

(77) Hsu, S.H., Huang, T.B., Cheng, S.J. et al. (2011) Chondrogenesis from human placenta-derived mesenchymal stem cells in three-dimensional scaffolds for cartilage tissue engineering. Tissue Engineering Part A, 17 (11-12), 1549-1560.

(78) Spadaccio, C., Rainer, A., Trombetta, M. et al. (2009) Poly-L-lactic acid/hydroxyapatite electrospun nanocomposites induce chondrogenic differentiation of human MSC. Annals of Biomedical Engineering, 37 (7), 1376-1389.

(79) Schofer, M.D., Boudriot, U., Leifeld, I. et al. (2009) Characterization of a PLLA-collagen I blend nanofiber scaffold with respect to growth and osteogenic differentiation of human mesenchymal stem cells. Thescientificworldjournal [Electronic Resource], 9, 118-129.

(80) Schofer, M.D., Tunnermann, L., Kaiser, H. et al. (2012) Functionalisation of PLLA nanofiber scaffolds using a possible cooperative effect between collagen type I and BMP-2: impact on colonization and bone formation in vivo. Journal of Materials Science. Materials in Medicine, 23 (9), 2227-2233.

(81) D'Angelo, F., Armentano, I., Cacciotti, I. et al. (2012) Tuning multi/pluri-potent stem cell fate by electrospun poly(L-lactic acid)-calcium-deficient hydroxyapatite nanocomposite mats. Biomacromolecules, 13 (5), 1350-1360.

(82) Ravichandran, R., Venugopal, J.R., Sundarrajan, S. et al. (2012) Precipitation of nanohydroxyapatite on PLLA/PBLG/Collagen nanofibrous structures for the differentiation of adipose derived stem cells to osteogenic lineage. Biomaterials, 33 (3), 846-855.

(83) Yang, F., Murugan, R., Ramakrishna, S. et al. (2004) Fabrication of nano-structured porous PLLA scaffold intended for nerve tissue engineering. Biomaterials, 25 (10), 1891-900.

(84) He, L., Liao, S., Quan, D. et al. (2010) Synergistic effects of electrospun PLLA fiber dimension and pattern on neonatal mouse cerebellum C17.2 stem cells. Acta Biomaterialia, 6 (8), 2960 2969.

(85) Yu, H.S., Won, J.E., Jin, G.Z., and Kim, H.W. (2013) Construction of mesenchymal stem cell-containing collagen gel with a macrochanneled polycaprolactone scaffold and the flow perfusion culturing for bone tissue engineering. BioResearch Open Access, 1 (3), 124-136.

(86) Gandhimathi, C., Venugopal, J., Ravichandran, R. et al. (2013) Mimicking nanofibrous hybrid bone substitute for mesenchymal stem cells differentiation into osteogenesis. Macromolecular Bioscience, 13 (6), 696-706.

(87) Lu, Z., Roohani-Esfahani, S.I., Kwok, P.C., and Zreiqat, H. (2011) Osteoblasts on rod shaped hydroxyapatite nanoparticles incorporated PCL film provide an optimal osteogenic niche for stem cell differentiation. Tissue Engineering Part A, 17 (11-12), 1651-1661.

(88) Nitya, G., Nair, G.T., Mony, U. et al. (2012) In vitro evaluation of electrospun PCL/nanoclay composite scaffold for bone tissue engineering. Journal of Materials Science. Materials in Medicine, 23 (7), 1749-1761.

(89) Phipps, M.C., Clem, W.C., Grunda, J.M. et al. (2012) Increasing the pore sizes of bonemimetic electrospun scaffolds comprised of polycaprolactone, collagen I and hydroxyapatite to enhance cell infiltration. Biomaterials, 33 (2), 524-534.

(90) Marino, G., Rosso, F., Ferdinando, P. et al. (2012) Growth and endothelial differentiation of adipose stem cells on polycaprolactone. Journal of Biomedical Materials Research. Part A, 100 (3), 543-548.

(91) Singh, S., Wu, B.M., and Dunn, J.C. (2011) Accelerating vascularization in polycaprolactone scaffolds by endothelial progenitor cells. Tissue Engineering Part A, 17 (13-14), 1819-1830.

(92) Capkin, M., Cakmak, S., Kurt, F.O. et al. (2012) Random/aligned electrospun PCL/PCLcollagen nanofibrous membranes: comparison of neural differentiation of rat AdMSCs and BMSCs. Biomedical Materials, 7 (4), 045013. 
(93) Jiang, X., Cao, H.Q., Shi, L.Y. et al. (2012) Nanofiber topography and sustained biochemical signaling enhance human mesenchymal stem cell neural commitment. Acta Biomaterialia, 8 (3), 1290-1302.

(94) Horne, M.K., Nisbet, D.R., Forsythe, J.S., and Parish, C.L. (2010) Three-dimensional nanofibrous scaffolds incorporating immobilized BDNF promote proliferation and differentiation of cortical neural stem cells. Stem Cells and Development, 19 (6), 843-852.

(95) Hwang, N.S., Varghese, S., Zhang, Z., and Elisseeff, J. (2006) Chondrogenic differentiation of human embryonic stem cell-derived cells in arginine-glycine-aspartate-modified hydrogels. Tissue Engineering, 12 (9), 2695-2706.

(96) Hwang, N.S., Varghese, S., and Elisseeff, J. (2007) Cartilage tissue engineering: Directed differentiation of embryonic stem cells in three-dimensional hydrogel culture. Methods in Molecular Biology, 407, 351-373.

(97) Liu, Z., Xiao, L., Xu, B. et al. (2012) Covalently immobilized biomolecule gradient on hydrogel surface using a gradient generating microfluidic device for a quantitative mesenchymal stem cell study. Biomicrofluidics, 6 (2), 024111-1-024111-12.

(98) Liu, S.Q., Tian, Q., Hedrick, J.L. et al. (2010) Biomimetic hydrogels for chondrogenic differentiation of human mesenchymal stem cells to neocartilage. Biomaterials, 31 (28), 7298-7307.

(99) McCall, J.D., Luoma, J.E., and Anseth, K.S. (2012) Covalently tethered transforming growth factor beta in PEG hydrogels promotes chondrogenic differentiation of encapsulated human mesenchymal stem cells. Drug Delivery and Translational Research, 2 (5), 305-312.

(100) Gandavarapu, N.R., Mariner, P.D., Schwartz, M.P., and Anseth, K.S. (2013) Extracellular matrix protein adsorption to phosphate-functionalized gels from serum promotes osteogenic differentiation of human mesenchymal stem cells. Acta Biomaterialia, 9 (1), 4525-4534.

(101) Munoz-Pinto, D.J., Jimenez-Vergara, A.C., Hou, Y. et al. (2012) Osteogenic potential of poly(ethylene glycol)-poly(dimethylsiloxane) hybrid hydrogels. Tissue Engineering Part A, 18 (15-16), 1710-1719.

(102) Ni, P., Fu, S., Fan, M. et al. (2011) Preparation of poly(ethylene glycol)/polylactide hybrid fibrous scaffolds for bone tissue engineering. International Journal of Nanomedicine, 6, 3065 3075 .

(103) Royce Hynes, S., McGregor, L.M., Ford Rauch, M., and Lavik, E.B. (2007) Photopolymerized poly(ethylene glycol)/poly(L-lysine) hydrogels for the delivery of neural progenitor cells. Journal of Biomaterials Science, Polymer Edition, 18 (8), 1017-1030.

(104) Liu, H.W., Chen, C.H., Tsai, C.L. et al. (2007) Heterobifunctional poly(ethylene glycol)tethered bone morphogenetic protein-2-stimulated bone marrow mesenchymal stromal cell differentiation and osteogenesis. Tissue Engineering, 13 (5), 1113-1124.

(105) Kobel, S.A. and Lutolf, M.P. (2012) Fabrication of PEG hydrogel microwell arrays for highthroughput single stem cell culture and analysis. Methods in Molecular Biology, 811, 101-112.

(106) Tang, J., Peng, R., and Ding, J. (2010) The regulation of stem cell differentiation by cell-cell contact on micropatterned material surfaces. Biomaterials, 31 (9), 2470-2476.

(107) Yang, I.H., Co, C.C., and Ho, C.C. (2011) Controlling neurite outgrowth with patterned substrates. Journal of Biomedical Materials Research. Part A, 97 (4), 451-456.

(108) Moeller, H.C., Mian, M.K., Shrivastava, S. et al. (2008) A microwell array system for stem cell culture. Biomaterials, 29 (6), 752-763.

(109) Sakai, Y., Yoshiura, Y., and Nakazawa, K. (2011) Embryoid body culture of mouse embryonic stem cells using microwell and micropatterned chips. Journal of Bioscience and Bioengineering, 111 (1), 85-91.

(110) Schukur, L., Zorlutuna, P., Cha, J.M. et al. (2013) Directed differentiation of size-controlled embryoid bodies towards endothelial and cardiac lineages in RGD-modified poly(ethylene glycol) hydrogels. Advanced Healthcare Materials, 2 (1), 195-205. 


\title{
12
}

\section{Practicalities to Translation from the Clinic to the Market}

\author{
Devyn M. Smith \\ Neusentis Research Unit, Pfizer Worldwide R\&D, UK
}

\subsection{Introduction}

Stem cells have been used successfully for over 50 years in the treatment of patients in the context of bone-marrow transplant [1]. However, over the last 15 years the use of stem cells as potential therapies has been subject to both high levels of hype and a lack of significant commercial success, since the first embryonic stem cells were discovered in the late 1990s [2]. While regenerative medicine is a billion-dollar industry today, it is still quite small given a single drug can generate many billions of dollars in revenue [3]. This apparent lack of commercial success could be attributed to a variety of factors, including lack of significant funding, limited success of clinical trials, uncertainty around business models [4], and an overall misunderstanding of stem-cell biology by the public. However, there are some key learnings that can be culled from the regenerative medicine industry over the last 15 years. This chapter will focus on addressing some questions surrounding these, including:

1. How does the process of commercializing stem-cell products differ from that of biopharmaceutical products or medical devices?

2. Over the last 15 years, what cell-based therapies have been commercialized?

3. What are the key learnings (both good and bad) that can be gained from analyzing these therapies?

Chemical Biology in Regenerative Medicine: Bridging Stem Cells and Future Therapies, First Edition. Edited by Charles C. Hong, Ada S. Ao, and Jijun Hao.

(C) 2014 John Wiley \& Sons, Ltd. Published 2014 by John Wiley \& Sons, Ltd. 
4. What are the special challenges to commercialization of stem-cell therapies?

5. What are the key learnings for a scientist to keep in mind as they move an asset through from concept to the clinic to commercialization?

6. What is the right strategy for translating a new therapy into the clinic and beyond?

7. What are the key activities to consider for successful translation of a concept into a company?

\subsection{Commercialization Comparison with Small Molecules, Medical Devices, and Biologics}

A key question is: How does commercialization of cell-based products differ from commercialization of other traditional medical therapies? We can evaluate three major categories of medical therapies: small molecules, biologics (large molecules), and medical devices. Small molecules have been commercialized for years using traditional approaches in marketing, such as hiring large numbers of sales reps to detail physicians (both primary care and specialists) in order to provide widespread information about the product [5]. The key to success is strong marketing, competitive differentiation from other products, and low cost of goods (COG), which can drive both revenue and profitability [6]. While biologics have traditionally been focused on specialist markets, given their overall higher pricing, the detailing and push to reduce COG are critical for profitability and the overall model is not significantly different from that of small molecules. The primary difference is that most biologic products must usually be kept cold (storage temperatures vary), which presents important logistical considerations versus small molecules. Reimbursement for both types of product is similar, although biologics are often more expensive than small molecules. In addition, both biologic- and small molecule-based products can cost many hundreds of millions of dollars and can take 10 or more years from early discovery to market launch [7]. However, once an asset is on the market, it enjoys exclusivity for a period of time, until patent expiry (which in some cases can be 10 or more years post-launch). While loss of market exclusivity typically results in a small-molecule product losing $80 \%$ or more of revenue within a year or two, biologics have yet to be significantly impacted by loss of exclusivity. In fact, although several biologics have experienced patent expiry there are few launched generics or biosimilars on the market, with those that have launched experiencing minimal commercial revenue [8].

Medical devices are typically complex pieces of metal and/or plastic sold primarily to surgeons or other specialists. Companies selling these devices utilize a different commercialization model from that for small and large molecules. Medical devices typically have higher COG than small molecules or biologics [9]. There are several reasons for this, including the higher production costs, relatively lower selling price, and need for more complex sales-force detailing. A typical medical-device sales rep will often be present and assist in the training of physicians in the use of the device, which builds a strong relationship with the physician but also results in higher costs for the selling company [10]. In addition, while the product lifecycle for biopharmaceuticals is long, that for a medical device is quite short, given the perpetual innovation that occurs in this space. This means companies must rapidly develop new products without the long R\&D cycles common with biopharmaceuticals. Given the very different business models and COG, there are few companies that 
sell products which use both the device and the traditional drug model. Johnson \& Johnson $(\mathrm{J} \& \mathrm{~J})$ is the one large-company exception, as it has been successful selling both medical devices and traditional small-molecule and biologic products [11]. Most other companies in the space are focused on either traditional biopharmaceuticals or medical devices.

The models described here are well-established in the market, such that physicians, sales reps, manufacturers, payers, and even patients are comfortable with them, despite their differences. How does cell-based therapy fit into this? Such therapies can possess elements from both the biopharmaceutical model and the medical-device model [12]. For example, a cell-based therapy will likely require some type of cold-chain distribution, not unlike biologics. However, it may require more complex provisioning and hence a field force that is more akin to that for a medical device. This mix of models makes cellbased therapies problematic for both traditional medical-device manufacturers who are not familiar with cold-chain logistics and distribution and biopharmaceutical companies that are not familiar with the complex sales and marketing requirements of devices, not to mention the relatively lower gross margins. In addition, the business model will differ between an autologous and an allogeneic therapy [13]. We will not delve into the business-model and scientific differences between allogeneic and autologous therapies, as this has been covered elsewhere $[4,9,12,13]$. However, $\mathrm{J} \& \mathrm{~J}$ demonstrates that it is possible for a company to be expert in both business models, and it is therefore likely that once commercial cell-based products are on the market, medical-device and biopharmaceutical companies will adapt to be successful in selling them.

\subsection{Historical Review and Case Studies}

There have been several cell-based products launched over the last 15 years $[9,12,13]$. Table 12.1 highlights the largest revenue-generating products that have launched. There were three key launches in the 1990s: Carticel (sold by Genzyme, now part of SanofiAventis), Dermagraft (launched by Advanced Tissue Sciences (ATS) and now sold by Advanced Biohealing (ABH)/Shire), and Apligraf (sold by Organogenesis). Each of these has had a storied history that has been quite challenging at times. Today, Dermagraft and Apligraf are both generating more than $\$ 100$ million in revenue, with double-digit growth, while Carticel has been selling $<\$ 50$ million for the better part of a decade. Dendreon recently launched Provenge' providing a more recent example that we can learn from. We will review Dermagraft (as representative of the other two products) and Provenge to evaluate what has gone right and what has not for these products since their launches.

\subsubsection{Dermagraft}

A detailed case study of Dermagraft was published in 2010 [14], covering the key aspects of the company, ATS, that initially developed and marketed the product. ATS filed for bankruptcy and was liquidated in 2003 after never making a profit in 14 years. This discussion will not replay the company's fate, given the prior published case study was thorough and informative. The key facts from this case study are:

- ATS was founded in 1987 as Marrow Tech Inc.

- In 1988 ATS raised \$6 million in an initial public offering (IPO). 
Table 12.1 Key cell-based products

\begin{tabular}{|c|c|c|c|c|c|}
\hline Company & Product & $\begin{array}{l}\text { Therapeutic } \\
\text { Area }\end{array}$ & Launch & $\begin{array}{l}2011 \text { WW } \\
\text { Revenue }\end{array}$ & $\begin{array}{l}\text { Growth } \\
\text { '10-'11 }\end{array}$ \\
\hline Dendreon & Provenge & Oncology & 2010 & $\$ 213 M$ & $344 \%$ \\
\hline $\begin{array}{l}\text { Advanced } \\
\text { Biohealing/ } \\
\text { Shire }\end{array}$ & Dermagraft & Skin & 1997 & $\sim \$ 200 M$ & $35 \%$ \\
\hline Organogenesis & Apligraf & Skin & 1998 & $\sim \$ 102 \mathrm{M}^{*}$ & $25 \% *$ \\
\hline Osiris/Nuvasive & $\begin{array}{l}\text { FormaGraft, } \\
\text { Osteocel Plus }\end{array}$ & Bone & 2005 & $\$ 99 M$ & $10 \%$ \\
\hline Genzyme/Sanofi & Carticel & Cartilage & 1995 & $\sim \$ 32 \mathrm{M}^{* *}$ & $<5 \%$ \\
\hline Cytori & Celution & $\begin{array}{l}\text { Soft Tissue } \\
\text { (adipose) }\end{array}$ & 2008 (ex-US) & $\sim \$ 8 \mathrm{M}$ & $0 \%$ \\
\hline Tigenix & ChondroCelect & Cartilage & 2009 (ex-US) & $\sim \sim \$ 1.6 \mathrm{M}$ & $85 \%$ \\
\hline
\end{tabular}

Cell Based \$665M Market in 2011 with 25\% + growth

$E=$ estimated from various sources, ${ }^{*}=$ data from $2010,{ }^{* *}=$ from Evaluate, Large Companies, rest of data from company $10 \mathrm{Ks}$

- ATS requested approval of Dermagraft TC (later called TransCyte) from the FDA. Dermagraft which was approved for burns in 1996.

- Smith \& Nephew signed an agreement worth \$10 million upfront and \$60 million in downstream payments for a joint venture to market Dermagraft in 1996.

- In 1998, the FDA refused to approve Dermagraft for diabetic foot ulcers.

- Dermagraft was subsequently approved in 2001 for the treatment of diabetic foot ulcers based on the submission of new clinical data.

- In 2002, ATS filed for Chapter 11 bankruptcy when it ran out of funds and sold the Dermagraft JV to Smith \& Nephew to continue supply of Dermagraft to patients [14].

It is clear that several key elements led to ATS's eventual failure as a company despite it having a launched product on the market. These include: a lack of focus on driving revenue, with a weak internal sales/reimbursement team; difficulty in marketing a novel and expensive product to a market used to relatively simple, inexpensive treatments such as dressings; and a lack of strategic focus, resulting in a lot of "wasted" R\&D spend. Dermagraft was eventually sold to $\mathrm{ABH}$ for $\$ 7$ million in 2006, and $\mathrm{ABH}$ relaunched the product and grew it to more than $\$ 100$ million in revenue by 2011 . ABH was recently purchased by Shire for $\$ 750$ million, highlighting the success of the relaunched Dermagraft [15]. Why was ABH so successful relative to ATS? The keys to its success are its focus on exactly the things that ATS failed to focus on - namely sales and reimbursement, creating a clear sales and marketing strategy to target key physicians, and making Dermagraft successful without spending significant $R \& D$ dollars on any other pipeline products.

\subsubsection{Provenge}

Dendreon Corporation is a biotech company headquartered in Seattle, Washington. It was founded in 1992. It took 18 years and \$1.2 billion (aggregate losses from 1992 to 2010, 
with another $\$ 750$ million from 2010 to 2012 post-launch) before its first product, Provenge (sipuleucel-T), was approved in April 2010 for the treatment of asymptomatic or minimally symptomatic metastatic castrate-resistant (hormone-refractory) prostate cancer [16]. Dendreon is focused on developing treatments for cancer using active cellular immunotherapy (ACI). ACI uses a patient's own white blood cells to destroy the tumor. In the case of Provenge, a patient's white blood cells are harvested using Leukapheresis and sent to the Dendreon manufacturing facility. The cells are then activated using a complex manufacturing process and returned to the infusion center to be infused back into the patient. The time from final manufacturing to infusion must be less than 18 hours, which can present logistical challenges for the company, patients, and physicians. Because of this short time window, Dendreon has built three manufacturing sites such that the entire United States is within a short flight or drive from one of them. The course of treatment is three infusions, one every 2 weeks for 6 weeks, and it has led to 4 months of improved survival in clinical studies [17].

When Provenge was approved, the expectations for the product were extremely high. In fact, some analysts forecasted peak revenue at $\$ 4$ billion [18]. However, 18 months later they were predicting no more than $\$ 500$ million in peak revenue from the drug [18]. What had changed? There were several factors, including the launch of new competitor drugs and commercialization and reimbursement challenges at Dendreon.

One of the competing drugs, Zytiga, was launched by J\&J in April 2011 for patients with late-stage (metastatic) castration-resistant prostate cancer in combination with prednisone (a steroid) who have received prior docetaxel (chemotherapy) [19]. Zytiga extends life expectancy 4-5 months and is estimated to generate $\$ 1.7$ billion in peak revenue [20]. In addition, Medivation and Astellas had Xtandi approved in September 2012. Zytiga is a small molecule and prices at $\$ 5500$ a month (\$66 000 annual), as versus Provenge's price of $\$ 93000$ for a course of treatment and Xtandi's $\$ 85000$ annually. Xtandi has peak forecasts at $\$ 2.2$ billion [21]. In effect, the market has moved quickly to small-molecule products that offer similar or better efficacy in a much simpler treatment procedure for both patients and providers.

Dendreon had a full year's lead on the competition but was not able to capitalize on this advantage. This was due to the commercialization and reimbursement challenges the company faced. Given the drug used a new business model, the company struggled to determine the optimal way to commercialize it. Physicians had to treat the patients and then be at risk of having to pay Dendreon the $\$ 93000$ cost of the drug if the insurance company did not reimburse them. This made them very nervous. Meanwhile, a patient who needed small-molecule therapy could get that from the pharmacy, with no financial risk for the physician. Medicare was willing to reimburse physicians for Provenge, but many physicians, particularly those in community practices, were wary. It has taken several years to educate these physicians about the reimbursement on offer [22]. In fact, the company had just begun to see an increase in the number of community physicians who use Provenge in the fourth quarter of 2012 [23]. This business-model challenge became an acute issue when the company warned in mid-2011 that it would not reach its guidance for 2011 revenue, resulting in a $60 \%$ drop in its stock price [24].

Another challenge for Dendreon involved manufacturing. As already mentioned, the company had built three manufacturing plants (New Jersey, Georgia, and California) to enable it to ship anywhere in the United States within the 18-hour product life. However, 
Table 12.2 Provenge gross margins

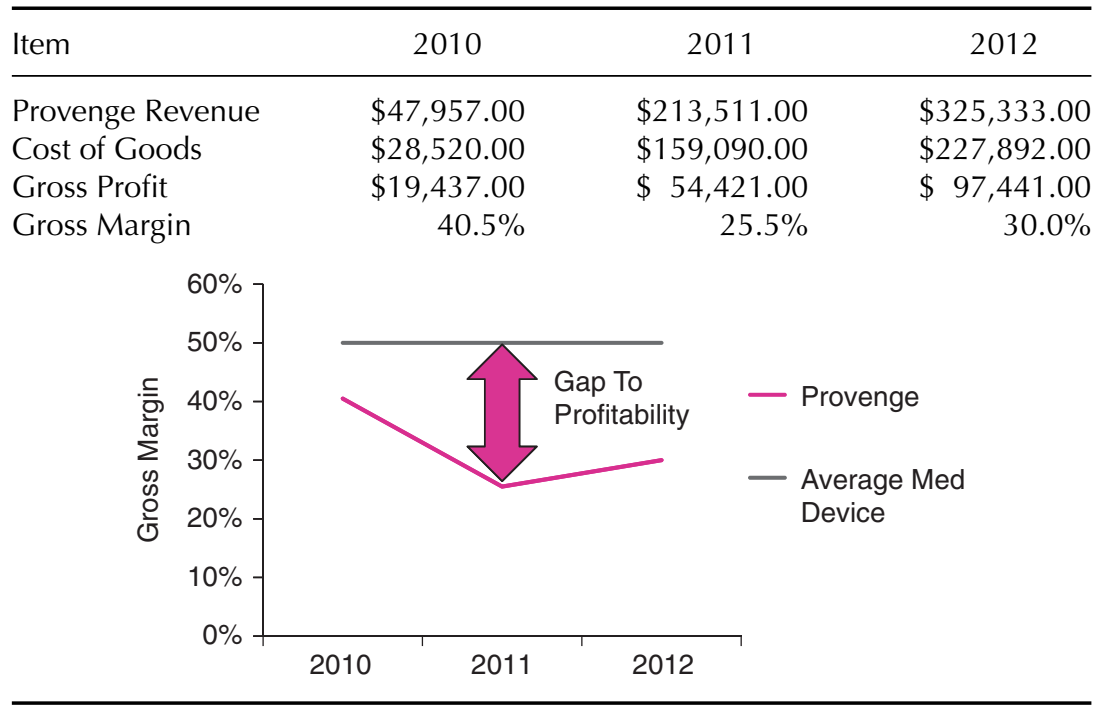

Source: Dendreon 10K filings - www.sec.gov

there was too much capacity, resulting in massive cash burn [25]. One plant was sold in an effort to improve gross margins as Dendreon attempted to reach profitability.

If we analyze the revenue achieved by year for Provenge, as compared against COG, we can see that the COG is not decreasing as one would hope (Table 12.2) [26]. This means that the company will struggle to be profitable in the near future. In fact, the gross margin only increased from 25 to $30 \%$, while the revenue was up nearly 50\% [26]. At this pace, the revenue would need to be over $\$ 1$ billion a year to obtain the margins necessary for profitability. However, Dendreon has announced that the recent restructurings should result in COG > 50\% by Q3 2013 and that they can achieve a positive cash flow at \$400 million in revenue [23].

These struggles have meant a significant amount of restructuring to drive costs down. In 2011, Dendreon laid off 500 employees, and another 600 were laid off in 2012. These efforts have decreased the number of employees from a high of 2000 in 2010 to 1100 in 2012. In addition, as already mentioned, in December 2012 Dendreon sold its New Jersey manufacturing facility to Novartis for $\$ 43$ million, transferring 100 employees to Novartis in the process [27]. These struggles have also had a tremendous impact on the company's share price (Table 12.3).

Where does that leave us at the date of writing, in early 2013? As already mentioned, the company has seen an increase in the use of Provenge by community physicians, which is a positive trend. Dendreon is also expecting regulatory decisions in Europe in 2013, which could also increase revenue, although not necessarily profitability [23]. In addition, the massive restructuring to drive down costs should begin to pay off later in 2013, as the company is much leaner now and expenses are much lower than they were over the last few years. Time will tell how successful Dendreon will be, but the company has struggled to date. 
Table 12.3 Dendreon share price and market capitalization

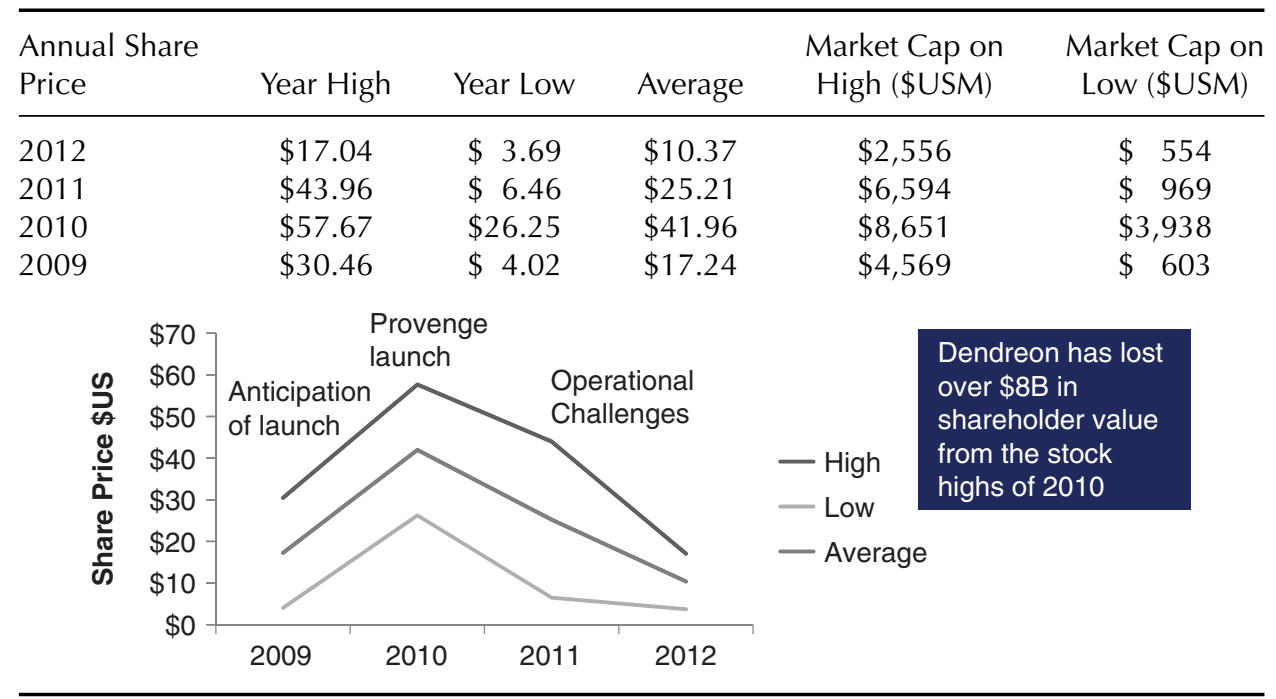

Source: Dendreon 10K filings - www.sec.gov

\subsection{Commercialization Challenges and How to Overcome Them}

From these case studies, some key themes emerge that are important to keep in mind for products in development:

- Strong, focused strategy: Focus on achieving commercial success with the lead asset or on getting the lead asset through clinical studies. Diluting the company's scarce capital on other noncritical studies of the lead asset or the development of other assets is poor allocation of capital.

- Strong financial oversight and strategy: Manage costs to ensure that spending is focused on activities that will most rapidly lead to clinical studies. While there may be nice-tohaves, focus resources on the must-haves.

- Focused manufacturing strategy: Ensure that spending on manufacturing and capacity matches the real expectations of the commercial organization. Bringing new capacity on-line "just in time" is critical to maximizing profitability.

- Strong sales strategy: For commercial assets, focus on increasing revenue via creation of a strong sales/reimbursement team, building relationships with payers, and understanding how to "sell" the product.

\subsection{Translation from the Bench to the Clinic: Key Considerations}

Let's take a step back from the commercialization considerations and focus on how to develop a product from idea to the clinic. A key question is what else we should keep in 
mind when translating a potential product from preclinical to clinical development. Other questions include:

- What indication should I pursue?

- What is my product?

- Should I worry about the competition?

- What is my partnering strategy?

- How/where do I manufacture GMP material?

- What is the regulatory environment like?

- How do I fund the product development?

- When do I start my company?

While all of these questions do not need to be answered in detail, knowing the rough answers to each is critical for raising funds from investors and creating a successful product. In effect, the answers to these questions become the core of the business plan and business strategy for the new company. Ensuring a company has a robust strategy is critical to success. Each of these key questions is discussed in detail in the remainder of the chapter.

\section{What indication should I pursue?}

Selection of a lead indication for the cell-based therapy is one of the most important considerations for moving into the clinic. The current crop of cell-based therapies is spread across multiple indications, with the majority focused in ischemic and autoimmune indications [28]. Are these the best indications to be pursuing, or is this merely the result of all companies chasing the same indications (i.e. a lemming effect)? Key considerations when selecting an indication are: Is there a scientific rationale for this indication? Is there preclinical efficacy in an animal model (or multiple animal models)? Is there clinical efficacy with similar products? Is this an indication for which a relatively expensive cell-based treatment could be used and reimbursed? While the first three questions are relatively straightforward and driven by available scientific data, the last one bears further discussion. First, expensive treatments have historically been reserved for diseases with significant morbidity and/or mortality, such as cancer and rheumatoid arthritis. In these diseases, expensive biologic therapies (costing in the $\$ 20000$ + /year range) are now well established in standard treatment regimens [29]. In fact, Dendreon's cell-based therapy Provenge is priced at $\$ 93000$ for a treatment regimen in cancer [30]. In addition, orphan diseases have emerged as an area where extremely highly priced therapies have now emerged, some costing more than $\$ 100$ 000 a year [31]. Why can these therapies command this type of pricing? Because of the serious morbidity and mortality associated with these diseases and the significant positive impact these therapies can have on patients.

This suggests that cell-based therapies are likely best placed in diseases with high unmet need and significant mortality, such as heart attack/heart failure, cancer, neurodegenerative diseases such as amyotrophic lateral sclerosis (ALS), and orphan diseases. These disease states are much better starting points for a cell-based therapy than diseases that either impact a large number of patients (e.g. diabetes) or have relatively low unmet need (e.g. allergic rhinitis), where a relatively highly priced therapy would be likely to fail. In addition, once a target indication is decided on, the end customer for the product (i.e. the treating physician) 
is clear. Having discussions with these treating physicians is critical to ensuring that the cell-based therapy meets their patients' needs. This work can be used to create what is called a target product profile in the biopharma industry. A target product profile spells out who the target patient is, who the treating physician is, what the current treatment standard is, the current standard's efficacy and safety profiles, and the target safety and efficacy profile of the new cell-based therapy - all of which can be communicated on a single slide.

Finally, one pitfall that many companies fall into is the temptation to target multiple disease areas at once. Often, as we saw in our case studies, this will lead to a lack of focus and an inability to deliver on the lead program while burning through capital, often the scarcest resource for a small company. As seductive as it may seem to show a full pipeline of indications, multiple simultaneous programs should be avoided until significant funding is achieved.

\section{What is the product?}

This may seem like a simple question, but it is key to success. The product could be the cells, the process, the manufacturing process, a device to isolate cells, among other things. Defining what the product is can enable IP and competitive differentiation. Some key elements to keep in mind include:

- Keep the product simple to use for the physician: it should not require significant work-up before being given to the patient, have relatively off-the-shelf usage, include a simple-to-use device if needed, and so on.

- Ensure that even if IP is difficult to achieve, there is significant proprietary know-how to keep competitors at bay.

- Have some competitive advantage over other cell-based therapies or treatments in the same disease. If efficacy is equal, is there a differentiation in safety, ease-of-use, time to onset, or need for redosing that can provide some differentiation in the market?

- Be sure that the product can match an existing business model. As discussed earlier, creating a new business model de novo is high-risk and often ends in product failure [32]. Keeping the product based on an existing successful business model will make the commercialization and partnering path much simpler [33].

- If a novel business model is required, make sure the product is clearly able to deliver transformational efficacy in the lead indication. Transformational efficacy means that the product provides substantial benefits over existing therapies (e.g. it is curative or greatly extends life).

- Understand the regulatory challenges that the product might encounter. For example, is it a device, cells only, or a combination of both? How do regulators in the United States versus the European Union versus Japan view your product? Is there a precedent for the regulatory path?

Answers to these questions may require discussions with treating physicians of the targeted disease, regulatory experts, and experts in product commercialization to ensure that the best product and optimal parameters are selected [34]. These discussions do not need to be expensive or extensive, but some investment is required to ensure success. 


\section{Should I worry about the competition?}

Understanding the competition is critical to a successful business plan and product. This extends beyond the cell-based competition to any therapy or device that is targeting the lead indication. The current treatment regimen, current products in the pipeline, current/future generic treatments, and so on should all be assessed across all therapeutic classes. Building this competitive map can enable one to successfully place the new cell-based therapy relative to the competition in the future treatment paradigm. For example, if the current treatment regimen is a generic small molecule followed by an antibody product then the new cell-based therapy is likely to be used after these two lines of therapy even if it is more efficacious, because most new therapies are applied in the last line of the treatment regimen and work their way toward firstline once efficacy and safety benefits relative to the other therapies are proven.

\section{What is my partnering strategy?}

The goal of most academics and newly formed companies is to obtain support from larger companies interested in licensing or providing funding to their idea/asset. While this is a worthy goal, it is critical to know what larger companies are interested in and whether the idea/asset is a good fit with their portfolio and strategy. As highlighted in a 2010 publication [35], large companies have made some plays in the regenerative medicine space, but these are primarily limited to the use of stem cells as tools and cell-based products that are already in the clinic or commercialized. This suggests that many efforts to attract a partner early in development will be unsuccessful, although making a large company aware of an asset and meeting the right business development people are worthy goals at the early stages and can develop a foundation for a successful partnership in the future.

\section{How/where do I manufacture GMP material?}

Manufacturing and subsequent impacts on COG cannot be overemphasized, as these can result in product failure. Finding a good manufacturing partner early is important. This partner could be a company, academic institution, or government-funded entity like the UK Cell Therapy Catapult [36]. The key is to find the right partner who can help in process development, lowering of COG, and the provision of a robust GMP facility to support the clinical trials. Saving a few dollars on manufacturing may seem like a good idea, but it might be a poor decision in the long-term. In addition, doing all manufacturing in-house might be a good idea, but often finding a partner to help is simpler, less expensive, and results in fewer problems as the therapy enters clinical trials. However, many companies have chosen to manufacture their own products, which can work if they have the financial resources.

\section{What is the regulatory environment like?}

There are many questions that must be addressed in the regulatory environment, including:

- Is there precedent for your therapy?

- Have similar therapies entered clinical trials?

- For approved therapies in the lead indication, what was the regulatory path taken?

- How large and how long must clinical trials must be? 
- What are the key monitoring requirements from the regulatory authorities?

- How does the regulatory environment differ by geography?

- In which geographic area do you want to start clinical trials?

- In which geographic area do you want to achieve first approval?

- What are the regulatory requirements related to manufacturing, product release, ongoing monitoring, and so on?

While the answers to some of these questions may not be known, creating a strong regulatory strategy is important for success. This may require hiring a regulatory expert as a consultant to ensure success.

\section{How do I fund product development?}

Product development from preclinical work to clinical trials is an expensive process and requires significant funding. It is important to know how much funding is required for each step and where it can be achieved. The funding plan should allocate appropriate resources for each milestone (e.g. up to preclinical, preclinical to FIH, FIH-Phase II, etc.). It should include all of the relevant experimental, manufacturing, regulatory, and clinical activities and spend required to reach each milestone. This will determine how much money needs to be raised as each stage. Funders, such as venture capitalists, will typically fund a company to the next milestone. If the milestone is hit then more funding can be released, whereas if it is not hit the company usually disappears. Funding can be generated from a variety of sources. Early on, grants from nonprofit or government institutions make up the bulk of funding. Once a technology or asset is more mature, angel funding or venture funding can be attained. Once clinical data is achieved, large companies (e.g. pharmaceutical or medicaldevice) might provide funding or an IPO could occur. An IPO results in the sale of a portion of the company to the public capital markets, where the shares are then traded. However, the IPO route has been severely curtailed during the recent financial crisis. Small companies have had more recent success with either selling the company or forming a partnership to garner new funding rather than attempting an IPO, particularly in the regenerative medicine space [37].

\section{When do I start my company?}

Timing is everything in the formation of a company. If we assume the regenerative medicine technology is emerging from an academic institution, many make the mistake of starting a company too early, when the technology is quite immature. This changes the entire dynamic regarding ability to get nonprofit or government funding, and investment is required to set up the infrastructure for the company that would be better spent on developing the technology. When the technology is nearing clinical trial, this is a good time to consider starting a company. Funding is usually easier to achieve at this stage, given the technology is more mature.

\subsection{Conclusion}

In this chapter we have reviewed the key considerations for starting a new regenerative medicine company and for translating products from idea to the clinic, and subsequently to commercial success. The final key to success is obvious: a therapy must provide significant 
benefit to patients. This is what companies in the drug-discovery and regenerative-medicine industry are trying to develop. The next 10 years will be exciting as we watch new regenerative therapies launch to the market, providing lifesaving benefits to patients.

\section{References}

(1) de la Morena, M.T. and Gatti, R.A. (2010) A history of bone marrow transplantation. Immunology and Allergy Clinics of North America, 30 (1), 1-15.

(2) Thomson, J.A., Itskovitz-Eldor, J., Shapiro, S.S. et al. (1998) Embryonic stem cell lines derived from human blastocysts. Science, 282 (5391), 1145-1147.

(3) Mason, C., Brindley, D.A., Culme-Seymour, E.J., and Davie, N.L. (2011) Cell therapy industry: billion dollar global business with unlimited potential. Regenerative Medicine, 6, 265-272.

(4) Driscoll, D. (2011) Addressing business models, reimbursement, and cost of goods. BioProcess International, 9 (S1), 46-49.

(5) Archstone Consulting. Launching new products in the post-blockbuster era: three practices from the industry's best. (no date) Available from: http://www.archstoneconsulting.com/ industries/life-sciences/white-papers/launching-new-products-three-practices.jsp (last accessed March 12, 2014).

(6) Strohl, W.R., Vitae, A., and Knight, D.M. (2009) Discovery and development of biopharmaceuticals: current issues. Current Opinion in Biotechnology, 20 (6), 668-672.

(7) Vernon, J.A., Golec, J.H., and Dimasi, J.A. (2010) Drug development costs when financial risk is measured using the Fama-French three-factor model. Health Economics, 19(8), 1002-1005.

(8) Denoon, A. and Vollebregt, E. (2010) Can regenerative medicine save Big Pharma's business model from the patent cliff? Regenerative Medicine, 5 (5), 687-690.

(9) Smith, D.M. (2012) Assessing commercial opportunities for autologous and allogeneic cellbased products. Regenerative Medicine, 7 (5), 721-732.

(10) Best Practices, LLC (2012) Medical device marketing. Available from: http://www. slideshare.net/bestpracticesllc/psm-272-a-medical-device-marketing (last accessed March 12, 2014).

(11) Johnson, M. (2011) Business model innovation at Johnson and Johnson - interview of Sheri McCoy (Vice Chairman, Executive Committee, Johnson \& Johnson). Available from: http:// www.frontendofinnovationblog.com/2011/05/fei-day-2-business-model-innovation-at.html (last accessed March 12, 2014).

(12) Smith, D.M. (2008) Successful business models for cell-based therapies, in The World Stem Cell Report 2008 (ed. B. Siegal), Genetics Policy Institute, pp. 158-162.

(13) Mason, C. and Dunnill, P. (2009) Assessing the value of autologous and allogeneic cells for regenerative medicine. Regenerative Medicine, 4, 835-853.

(14) Pangarkar, N., Pharoah, M., Nigam, A. et al. (2010) Advanced tissue sciences inc.: learning from the past, a case study for regenerative medicine. Regenerative Medicine, 5 (5), 823-835.

(15) Shire (no date) History of mergers and acquisitions. Available from: http://www.shire.com/ shireplc/en/business/acquisitions/acquisitionshistory (last accessed March 12, 2014).

(16) Malarkey, M.A. and Witten, C.M. (no date) Letter to Elizabeth C. Smith, Vice President of Regulatory Affairs, Dendreon Corporation, Seattle from Office of Cellular, Tissue and Gene Therapies, Center for Biologics Evaluation and Research, Food and Drug Administration. Available from: http://www.fda.gov/BiologicsBloodVaccines/CellularGeneTherapyProducts/ ApprovedProducts/ucm210215.htm (last accessed March 12, 2014).

(17) Dendreon Web site: http://www.dendreon.com (last accessed March 12, 2014).

(18) Pierson, R. (2011) Zytiga creeps up on Provenge prostate-cancer drug. Available from: http://www.reuters.com/article/2011/10/07/us-dendreon-zytiga-idUSTRE7965JF20111007 (last accessed March 12, 2014).

(19) Jefferson, E. (2011) FDA approves Zytiga for late-stage prostate cancer. Available from: http://www.fda.gov/NewsEvents/Newsroom/PressAnnouncements/ucm253055.htm (last accessed March 12, 2014). 
(20) Edney, A. (2012) J\&J's Zytiga wins approval for early prostate cancer use. Available from: http://www.bloomberg.com/news/2012-12-10/j-j-s-zytiga-wins-approval-for-earlier-usein-prostate-cancer.html (last accessed March 12, 2014).

(21) Palmer, E. (2012) J\&J's Zytiga gets expanded approval that could double sales. Available from: http://www.fiercepharma.com/story/jjs-zytiga-gets-expanded-approval-coulddouble-sales/2012-12-10 (last accessed March 12, 2014).

(22) Kumar, N.S. (2012) Dendreon: a case study in pharma. Available from: http:// www.mdmbas.com/Blog/files/922e707383c85cda6fd89d808b716901-7.html (last accessed March 12, 2014).

(23) Dendreon (2013) Dendreon Q4/FY 2012 earnings supplemental information February 25, 2013. Available from: http://files.shareholder.com/downloads/DNDN/0x0x638201/e05e4ade2367-496e-8501-cf82681c6e84/DNDN_Q4_FY_2012_Earnings_Supplemental_Information 00512855.PDF (last accessed March 12, 2014).

(24) McBride, G. (2011) Real world costs impacting Dendreon's Provenge sales. Available from: http://seekingalpha.com/article/298453-real-world-costs-impacting-dendreon-sprovenge-sales\#comments_header (last accessed March 12, 2014).

(25) Miller, D. (2010) Dendreon's dilemma isn't Provenge competition, demand - it's geography. Available from: http://www.minyanville.com/businessmarkets/articles/dendreon-dedreonguidance-provenge-johnson-and/11/8/2010/id/30996?page=full (last accessed March 12, 2014)

(26) Dendreon (2012) Form 10-K, annual report pursuant to Section 13 or 15(d) of the Securities Exchange Act of 1934, for the fiscal year ended December 31, 2012, Dendreon Corporation. Available from: http://www.sec.gov/Archives/edgar/data/1107332/000119312513074139/ d468268d10k.htm (last accessed March 12, 2014).

(27) Rocco, L. (2012) Dendreon sells New Jersey immunotherapy manufacturing facility. Available from: http://investor.dendreon.com/releasedetail.cfm?ReleaseID=728044 (last accessed March 12, 2014).

(28) Buckler, L. (2011) Late-stage industry-sponsored cell therapy trials. Available from: http://celltherapyblog.blogspot.com/2011/12/active-phase-iii-or-iiiii-cel-therapy.html (last accessed March 12, 2014).

(29) Miller, A. (2012) New pricing puts some "miracle drugs" out of reach. Available from: http://www.georgiahealthnews.com/2012/10/pricing-puts-miracle-drugs-reach/ (last accessed March 12, 2014).

(30) Timmerman, L. (2010) Dendreon sets Provenge price at $\$ 93000$, says only 2000 people will get it in first year. Available from: http://www.xconomy.com/seattle/2010/04/29/dendreonsets-provenge-price-at-93000-says-only-2000-people-will-get-it-in-first-year/ (last accessed March 12, 2014).

(31) Hooper, M. (2010) The world's most expensive drugs. Available from: http://www.forbes.com/ 2010/02/19/expensive-drugs-cost-business-healthcare-rare-diseases.html (last accessed March 12, 2014).

(32) Smith, D.M. (2009) Commercialization challenges associated with induced pluripotent stem cell-based products. Regenerative Medicine, 5, 593-604.

(33) Smith, D.M. (2009) Creating partnerships with large pharma? Getting ready for the big day, in The World Stem Cell Report 2009 (ed. B. Siegal), Genetics Policy Institute, pp. 131-137.

(34) Plagnol, A.D., Rowley, E., Martin, P., and Livesey, F. (2009) Industry perceptions of barriers to commercialization of regenerative medicine products in the UK. Regenerative Medicine, 4 , $549-559$.

(35) McKernan, R., McNeish, J., and Smith, D.M. (2010) Pharma's developing interest in stem cells. Cell Stem Cell, 6 (6), 517-520.

(36) Catapult Project Web site: https://catapult.innovateuk.org/cell-therapy (last accessed March 12, 2014).

(37) Thomas, D. (2012) The good, the bad and the ugly for biotech IPOs. Available from: http://www.biotech-now.org/business-and-investments/2012/11/the-good-the-bad-andthe-ugly-for-biotech-ipos (last accessed March 12, 2014). 


\section{Index}

Adipose stem cells, 155, 166

Adult stem cells (ASCs), 59-60, 71, 73

Alkaline phosphatase (ALP), 184, 189, 193

Blastocyst inner cell mass (ICM), 104-5, 108

Bone morphogenetic protein (BMP), 3, 19, 22, 67-71

Cardiac defects, 21, 22, 23

Cardiac iPSCs, 40, 50, 54, 95-7, 99, 101

Cardiac stem cell (CSC), 72

Cardiomyogenesis, animal models

Cellular and molecular events of, 3 , 19-20, 21-23, 26-27, 52, 55

Chemicals associated with, 40, 42, 55

Early stages of, 17-18, 20, 21, 23

EMT and cardiac remodeling, 8-9, 18, 20

MiRNA use in, 53

Morphological features of, 16-17

Cardiovascular progenitor cells, animal models

Different types of, 27-28

Directed differentiation of, 26-28

Regulation by Wnt signaling, 3-5, 9, 19-20

Cellular reprogramming, see iPSCs

Chemical genetics

Chemical cocktails for iPSCs, 37,

38-39, 98, 100, 142, 143-153
Chemicals used to induce cardiac regeneration, 40, 42, 55

Small molecules in regenerative medicine, 78, 80, 82-87, 96-101

Chemical screening, In silico, 169, 171-173

In vitro, 42, 169-171, 173

In vivo, zebrafish, 42-43

DNA methyltransferase inhibitors, 149, 164, 167

S-adenosylmethionine (SAM), 167, 168, 171-172, 173-176

ECM extracellular matrix (ECM), 182-183, 185-186, 188, 193

Ectoderm, 16, 19

Embryonic germ cells (EGCs), 105-106

Embryonic stem cells, animal models, 24

Embryonic stem cells (ESCs), human

Derivation of, 141

Heart repair using, 49

Limitations of, 50

Role of Wnt signaling, 2, 3, 25

Endoderm, 16, 19

Endothelial progenitor cell (EPC), 184, 191-193

Epiblast, 19

Epidermal growth factor (EGF), 59, $64-65,69,76$

Chemical Biology in Regenerative Medicine: Bridging Stem Cells and Future Therapies, First Edition. Edited by Charles C. Hong, Ada S. Ao, and Jijun Hao.

(C) 2014 John Wiley \& Sons, Ltd. Published 2014 by John Wiley \& Sons, Ltd. 
Epithelial-Mesenchymal Transition

(EMT)/Mesenchymal-Epithelial transition (MET), 38, 39

Extracellular matrix (ECM), 79-81, 87

Gastrulation, 16, 19

Growth factors, 78-88

Hair follicle stem cell (HFSC), 59-61, 62-3, 72

Hematopoietic stem cell (HSC), 7-8, 59-61, 66-68, 71-72

Histone deacetylase inhibitors in reprogramming, 146-147

Histone methyltransferase/demethylase inhibitors in reprogramming, 147-149, 164, 167-168

Intermediate progenitor cell (IPC), 59, 69, 71

Intestinal stem cell (ISC), 59, 63-65, $71-72$

iPSC reprogramming, 24, 95, 142

Chemicals used for, 37, 38-39, 98, 100 , $142,143-153,164,167-168$

Epigenetics barriers involved in, 145-146

In situ reprogramming approaches, 50, $51-53,54,55,101$

Limitations and challenges, 50, 52, 53-55, 142, 164

ROS generation in, 39, 154

Strategies for, 52, 142, 163

Transcription factors associated with, $24,36,37,38,142$

Label-retaining cell (LRC), 60, 62, 64 Leucine-rich repeat-containing GPCR5 (Lgr5), 60, 62, 64-5

Mesenchymal stem cell (MSC), 5-7, 180-194

Mesoderm, 16, 19

Myocardial infarction, 35, 49

Cell-based treatment of, 36, 44, 50

Cellular and molecular events, 41, 49, 50
Regeneration following, 41-42, 55

Paracrine effects from transplanted cells, 50

Inducing regeneration in situ, 51-53, 54

Neural progenitor cell (NPC), 187, 194

Neural stem cell (NSC), 60, 68-72, 147-148

Transdifferentiation from fibroblast, 155

Neural tube, 17

Platelet-derived growth factor (PDGF), 185

Pluripotency, maintenance of, 24, 25, 36-38

Pluripotent stem cell sources, 24, 35

Poly( $\varepsilon$-caprolactone) (PCL), 186-187, 191-193

Poly(D-lysine) (PDL), 188

Poly(dimethylsiloxane) (PDMS), 191-4

Poly(ethylene glycol) (PEG), 193-5

Poly(ethylene glycol) diacrylate (PEGDA), 188, 194-195

Poly(glycolic acid) (PGA), 192

Poly(2-hydroxyethyl methacrylate) (PHEMA), 188

Poly(lactic acid) (PLA), 191-192, 194-195

Poly(L-lactic acid) (PLLA), 186-187, 190, 192-193

Poly(lactic-co-glycolic acid) (PLGA), 183, 190-193

Primordial germ cells (PGCs), 105

Regenerative Medicine, 77-78, 80-82, 86-88

Subgranular zone (SGZ), 60, 68, 71, 73

Smooth muscle cell (SMC), 184, 185

Somatic cell nuclear transfer (SCNT), $106,141,142$

Stem cell niche, 8, 60-61

Transdifferentiation, 43, 50, 154, 155

Chemicals for, 155

Epigenetics during, 154, 155 
Mechanisms of, 43

Reducing teratoma formation, 51

Regenerate cardiac cells, 43-44, 50, 51-53

Transient amplifying cell (TAC), 60-2, 69-70

TGF-beta signaling, 150

Small molecule inhibitors of, 150, 151

Umbilical cord (UC), 186, 188

Ventricular-Subventricular Zone (V-SVZ), $60,68-70,73$
Wnt signaling, 1-2, 22, 151-152

Biphasic role in self-renewal and differentiation, 2, 3, 4, 7, 9

Cardiomyocyte differentiation, 3, 19, 22-23

Inhibitors of, 5, 42, 152-153

Maintenance of embryonic stem cells, 2, 151

Role in iPSC generation, 39, 151-152

Zebrafish,

Regeneration in, 40, 41, 77, 80 